
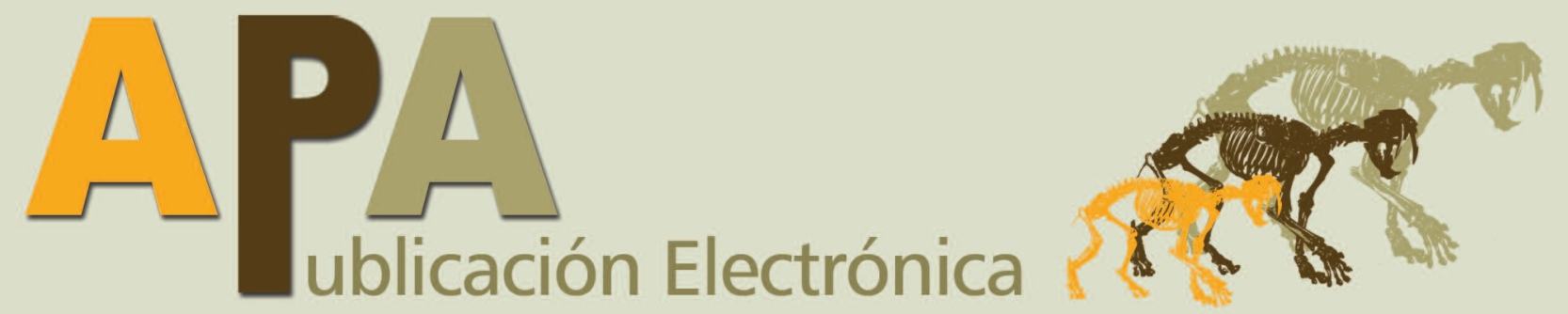

Reunión de Comunicaciones

de la Asociación Paleontológica Argentina, 2018

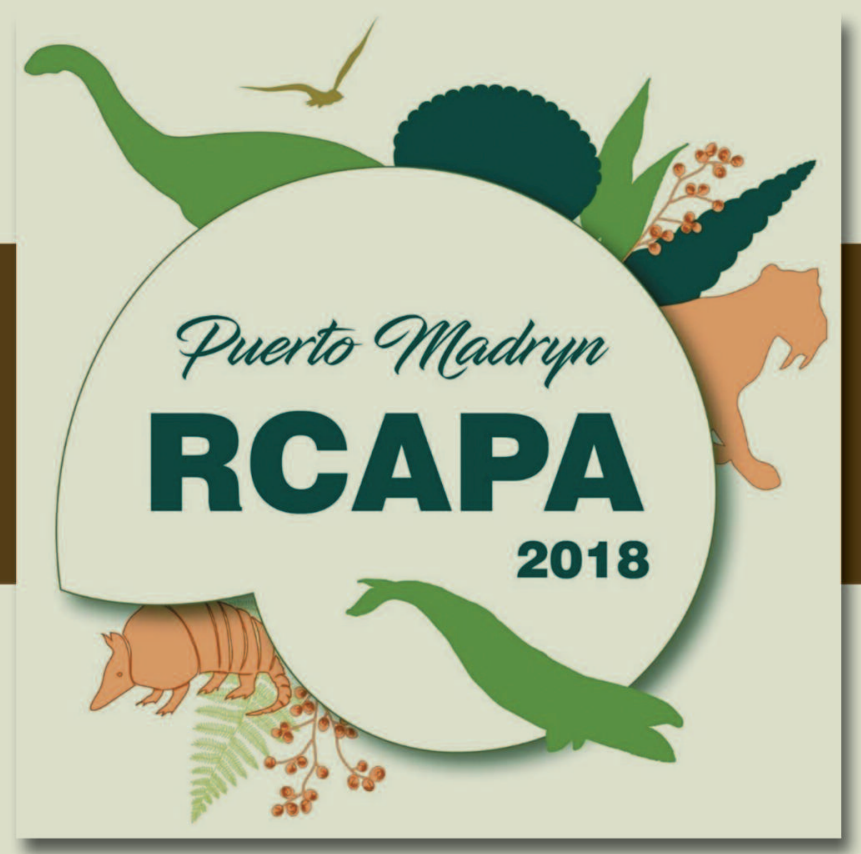

LIBRO DE RESÚMENES

21 al 23 de Noviembre 2018 


\title{
Reunión de Comunicaciones de la Asociación Paleontológica Argentina, 2018
}

\author{
LIBRO DE RESÚMENES
}

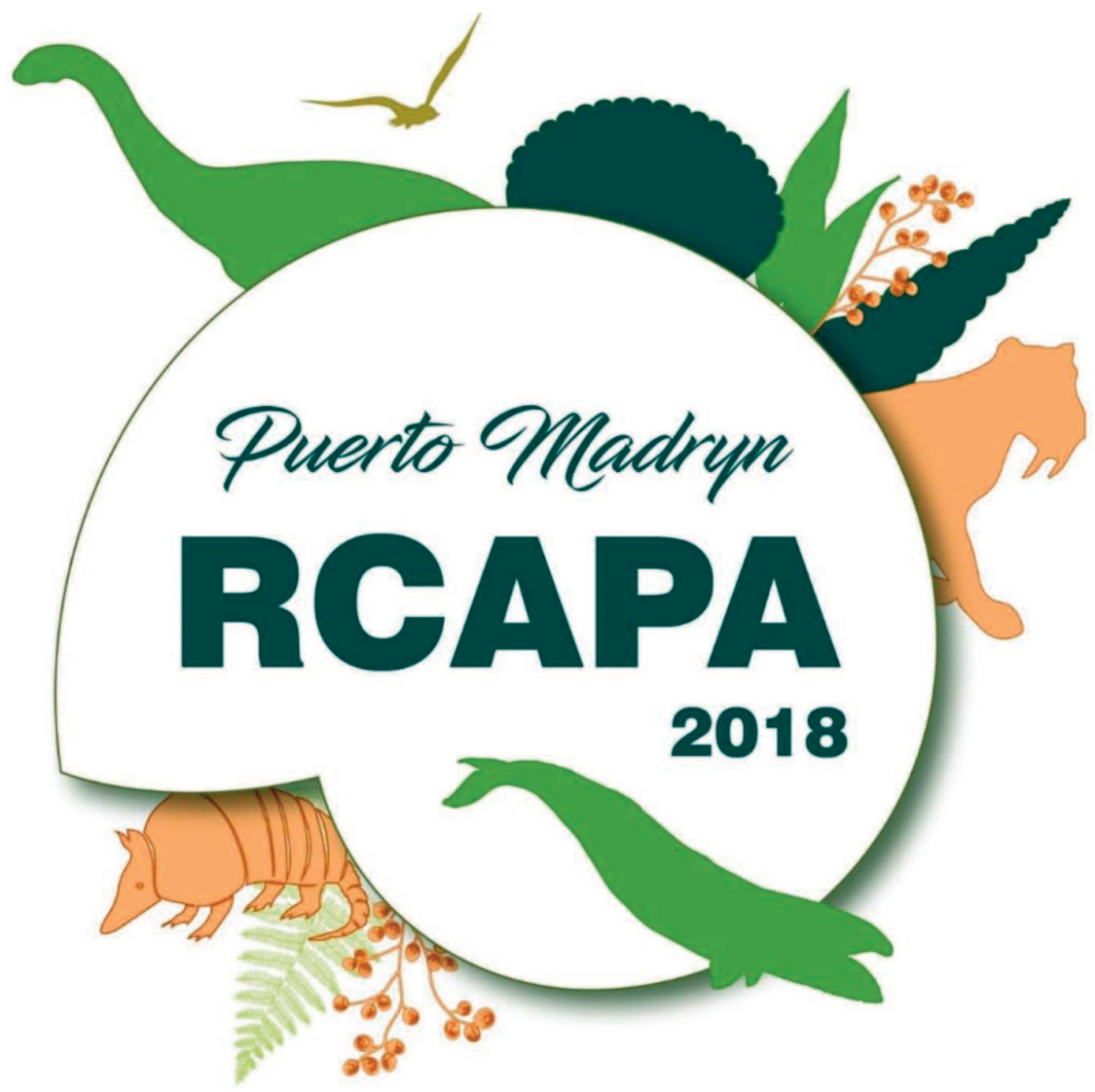

Puerto Madryn

21 al 23 de Noviembre 2018

Auspician

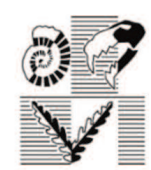

SECRETARIA do CIENCIA

TECNOLOGIA E INNOVACIÓN PRODUCTIVA de la PROVINCIA de CHUBUT
CONICET

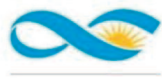

C E N P A T
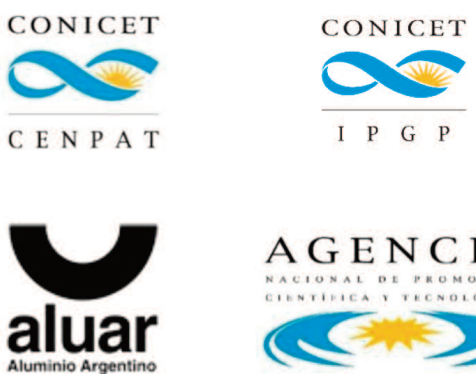

A G E N C I A (
M

MARBAR 


\title{
COMITÉ ORGANIZADOR (por orden alfabético)
}

\author{
Santiago Bessone \\ Mónica Buono \\ Felipe Busker \\ Nicolás Farroni \\ Mariel Ferrari \\ María Laura García Campos \\ Laureano González-Ruiz \\ Lucio Ibiricu \\ Ariel Méndez \\ Nelson Novo \\ Fernando Pap \\ Damián Pérez \\ Raúl Vacca \\ Mariana Viglino \\ Guadalupe Vilchez Barral
}

\section{COMITÉ CIENTÍFICO (por orden alfabético)}

\begin{abstract}
Dra. Mónica Romina Buono
Dr. Juan Ignacio Canale

Dr. José Luis Carballido
\end{abstract}

Dra. Graciela Andrea Concheyro

Dra. Penélope Cruzado-Caballero

Dra. Gabriela Catalina Cusminsky

Dra. Julia Brenda Desojo

Dr. Ignacio Díaz-Martínez

Dra. María Teresa Dozo

Dr. Ignacio Hernán Escapa

Dr. Martín Daniel Ezcurra

Dr. Juan Carlos Fernicola

\author{
Dra. Silvia Mariel Ferrari \\ Mg. Leonardo Sebastián Filippi \\ Dr. Federico Abel Gianechini \\ Sr. Jorge Antonio González \\ Dr. Lucio Manuel Ibiricu \\ Dr. Ari Iglesias \\ Tec. Marcelo Pablo Isasi \\ Dr. Darío Gustavo Lazo \\ Dr. Ariel Hernán Méndez \\ Dra. Laura Nicoli \\ Dra. Ariana Paulina-Carabajal \\ Dra. María Encarnación Pérez
}

\author{
Dr. Juan Pablo Pérez Panera \\ Tec. Pablo Puerta \\ Dr. Sebastián Miguel Richiano \\ Dra. Cecilia Rodríguez Amenabar \\ Dr. Leonardo Salgado \\ Dra. Juliana Sterli \\ Dr. Arturo César Taboada \\ Dra. Claudia Patricia Tambussi \\ Dr. Marcelo Franco Tortello \\ Dr. Ezequiel Ignacio Vera \\ Dr. Sergio Fabián Vizcaíno
}

\section{AGRADECIMIENTOS}

Desde la Comisión Organizadora de la RCAPA 2018 (Puerto Madryn) queremos agradecer a los siguientes auspiciantes y patrocinadores: Asociación Paleontológica Argentina (APA), CCT CONICET-CENPAT (autoridades y gerente), Instituto Patagónico de Geología y Paleontología (IPGP), Consejo Nacional de Investigaciones Científicas y Técnicas (CONICET), Agencia Nacional de Promoción Científica y Tecnológica, Secretaría de Ciencia, Tecnología e Innovación Productiva de la Provincia de Chubut, Consejo Federal de Inversiones (CFI), Ente Mixto de Promoción Turística de Puerto Madryn, Municipalidad de Puerto Madryn, Responsables del Bingo Municipal de Puerto Madryn, ALUAR y MARBAR. Asimismo, estamos profundamente agradecidos con el personal del CCT CONICET-CENPAT, particularmente con Fernando Pap y Renato Mazzini (SECECOM), Diego Nuñez de la Rosa y Alejandro Cannizzaro (Comunicación Institucional), Jorge Briguglio y Juan Saavedra (Personal de audiovisuales y salones), y Julio Rúa y Ricardo Vera (Área automotores y náutica) por toda la ayuda brindada durante la realización de la RCAPA. Queremos agradecer también a Jorge Antonio González y Patricio Ocampo Cornejo por la ayuda brindada en la coordinación de la muestra de Paleoarte. También agradecemos al comité científico, conferencistas y organizadores de simposios por su dedicación y buena predisposición. Finalmente, agradecemos a todos los participantes, que con más de 180 contribuciones de gran calidad científica y diversas temáticas, contribuyeron al éxito de la RCAPA 2018. 


\title{
CONFERENCIAS PLENARIAS
}

\section{BUSCANDO LOS TETRÁPODOS MÁS ANTIGUOS DE SUDAMÉRICA}

\author{
Juan Carlos Cisneros ${ }^{1}$ \\ ${ }^{1}$ Centro de Ciências da Natureza, Universidade Federal de Piauí, Teresina y Programa de Pós-Graduação em Geociências, Universidade Federal \\ de Pernambuco. Recife, Brasil.juan.cisneros@ufpi.edu.br
}

La Formación Pedra de Fogo en la Cuenca del Parnaíba (Pérmico: Cisuraliense), NE de Brasil, es conocida por su exuberante registro paleobotánico. Esta formación también ha aportado una fauna marina/costera, conocida desde los años 40, gracias a los trabajos de L.I. Price. Durante décadas, el único tetrápodo reportado en esta Formación era el temnospóndilo de rostro largo Prionosuchus plummeri, un Archaegosauridae de gran porte. En años recientes se llevaron a cabo una serie de campañas, cuyo resultado principal ha sido el descubrimiento de una nueva fauna de vertebrados en el norte de la cuenca, cerca de la ciudad de Teresina, Estado de Piauí. Esta fauna está asociada a un ambiente lacustre, en el cual también son encontrados troncos petrificados y microbialitos. Los vertebrados son colectados en pequeñas canteras en donde se explota limolita silicificada, la cual es utilizada para la construcción. La nueva fauna contiene temnospóndilos del clado Dvinosauridae, temnospóndilos rinesúquidos (su registro más septentrional), nuevos ejemplares de Prionosuchus plummeri, reptiles captorrínidos (primer registro en Sudamérica) y pararreptiles. Se registran también dipnoos, celacantos, diversos peces actinopterigios y algunos condrictios. Esta fauna continental se asemeja a las registradas en el sur de Estados Unidos, especialmente en Texas y Oklahoma. Algunos elementos florísticos y un condrictio (/tapyrodus) en común con la Formación Irati (Cuenca de Paraná), sugieren una edad de 278 Ma. Correlaciones paleoflorísticas con Chemnitz, en Alemania, sin embargo, sugieren 290 Ma, con lo cual los tetrápodos de Pedra de Fogo serían los más antiguos de Sudamérica.

\section{INVERTEBRADOS EN TIEMPO Y FORMA: CONCEPTOS Y PRECONCEPTOS EN HETEROCRONÍA}

\author{
JaVier ECheVARría ${ }^{1}$
}

${ }^{1}$ Consejo Nacional de Investigaciones Científicas y Tecnológicas (CONICET). División Paleozoología de Invertebrados, Museo de La Plata, Facultad de Ciencias Naturales y Museo, Universidad Nacional de La Plata. Paseo del Bosque s/n, B1900FWA La Plata, Buenos Aires, Argentina. javierechevarria@fcnym.unlp.edu.ar

La heterocronía (cambio evolutivo en los tiempos o tasas del desarrollo) puede generar cambios morfológicos profundos en un linaje, a la vez que favorece la convergencia. El estudio ontogenético y paleontológico de diferentes grupos de invertebrados ha acompañado el desarrollo histórico del concepto de heterocronía. La variación en la relación entre tamaño y forma (alometría) se emplea con frecuencia para reconocer la variación ontogenética; los organismos con crecimiento acrecional brindan buena información en ese sentido. Si bien el tamaño no puede equipararse a la edad al comparar entre especies, sigue siendo una buena aproximación en estudios intrapoblacionales. El estudio comparado de las alometrías entre especies permitirá reconocer patrones heterocrónicos, aunque no permita descifrar los procesos causantes de los mismos. Otro aspecto crítico es la necesidad de una hipótesis filogénetica. Sin embargo, el registro fósil puede ser también un buen contexto histórico para determinar la dirección de cambio heterocrónico: la aparición estratigráfica de las sucesivas ontogenias, ante un registro relativamente continuo como el de algunos invertebrados, puede ayudar a identificar el cambio heterocrónico aún sin filogenias precisas (aunque siempre subyace una hipótesis filogenética implícita). Además, el reconocimiento de potenciales convergencias puede llegar a ser incluso un prerrequisito para elucidar las relaciones filogenéticas en muchos casos. Finalmente, rara vez los procesos heterocrónicos son puros, sino que más comúnmente ocurren combinados y afectando de diferente manera a diferentes partes del organismo. La modularidad, en consecuencia, jugará también un papel importante en la evolución heterocrónica de los diferentes grupos de organismos. 


\section{VÍNCULOS GONDWÁNICOS EN LAS FLORAS DEL CRETÁCICO SUPERIOR Y PALEÓGENO DE AMÉRICA DEL SUR EN RELACIÓN CON LA DISTRIBUCIÓN PALEOGEOGRÁFICA Y LOS CLIMAS}

\section{ARI IGLESIAS ${ }^{1}$}

${ }^{1}$ Instituto de Investigaciones en Biodiversidad y Medioambiente (INIBIOMA), Consejo Nacional de Investigaciones Científicas y TecnológicasUniversidad Nacional del Comahue (CONICET-UNCO). Quintral 1250, R8400FRF San Carlos de Bariloche, Río Negro, Argentina.

ari_iglesias@yahoo.com.ar

Las floras Gondwánicas fueron extensamente distribuidas en la denominada Provincia Weddeliana. Aquí reviso el registro vegetal desde el Cretácico Superior hasta fines del Paleógeno del sur de América del Sur en relación con otras floras de Gondwana. Familias con registro desde el Cretácico son las Cunoniaceae, Myrtaceae, Nothofagaceae, Proteaceae, Winteraceae, Podocarpaceae, Araucariaceae. El pasaje K/Pg en Patagonia ocurre con una alta diversidad en las floras, demostrando una rápida recuperación de las asociaciones, diferente a otras regiones. Para el Paleógeno en Patagonia ocurren géneros típicos del trópico australásico que indican condiciones subtropicales húmedas en latitudes altas para América del Sur. Existen pocos registros de taxones del neotrópico actual, sin embargo se realzan los registros más antiguos de Astreaceae, Fabaceae, Menispermaceae y Solanaceae. El deterioro climático a partir del Eoceno superior y durante el Oligoceno, se ve reflejado en las floras. Muchos taxones tropicales se extinguen, algunos permanecieron en latitudes bajas; aunque hasta el Oligoceno medio es posible reconocer en la región andino-patagónica algunos que hoy caracterizan la región tropical de Brasil. A partir del Eoceno medio comienza en Patagonia la dominancia de Nothofagus, con una historia que se remonta al Cretácico Superior de la Península Antártica y el extremo sur de Patagonia; y culmina en la actual flora andino-patagónica. Pero aún en el Mioceno medio se reconocen floras bastante atípicas como la coexistencia de Nothofagus con palmeras, característica de climas mediterráneos en Chile y Nueva Guinea. 


\title{
CONTRIBUCIONES CIENTIFIICAS
}

\author{
SIMPOSIO “PALEOBIOLOGÍA Y EVOLUCIÓN \\ DE LOS DINOSAURIOS DE GONDWANA"
}

\section{VERTEBRAL PNEUMATICITY AMONG CERVICO-DORSAL TRANSITION OF NON-EUSAUROPODA SAUROPODOMORPHA}

\author{
C. Apaldetti ${ }^{1}$, R.N. Martinez ${ }^{1}$, and D. Pol ${ }^{2}$
}

${ }^{1}$ Centro de Investigaciones de la Geósfera y la Biósfera (CIGEOBIO)-Consejo Nacional de Investigaciones Científicas y Tecnológicas (CONICET)Instituto y Museo de Ciencias Naturales, Universidad Nacional de San Juan. Av. España 400 (Norte), J5400DNQ San Juan, San Juan, Argentina. capaldetti@unsj.edu.ar; martinez@unsj.edu.ar

${ }^{2}$ Museo Paleontológico Egidio Feruglio. Av. Fontana 140, U9100GYO Trelew, Chubut, Argentina. dpol@mef.org

Pneumatic structures among presacral vertebrae are present in the dorsal series of non-Sauropoda Sauropodomorpha (e.g., Eucnemesaurus, Aardonyx) and in the cervico-dorsal transition of Gravisauria and more derived Eusauropoda. Recently were known two non-eusauropod sauropodomorphs (the lessemsaurids Lessemsaurus and Ingentia) with pneumatic structures among their cervico-dorsal vertebral transition, which represent the earliest sauropodomorphs with pneumatic cervical vertebrae and/or cervico-dorsal transition. Here we present a new Sauropodiformes specimen (PVSJ 908) from the Late Triassic Quebrada del Barro Formation (Marayes-El Carrizal basin, Northwestern Argentina) with novel pneumatic structures in its cervico-dorsal vertebrae. This new pneumatic pattern includes the presence of deep fossae with internal subfossae (= internal chambers) on the pre and postzygapophyseal centrodiapophyseal fossa (prcdf and pocdf) on the posterior cervical (C9-10) and anterior dorsal (D1-5) vertebrae, which are unambiguously identified as pneumatic in origin. The presence of invasive pneumatic complex -fossae within fossae- observed among lessemsaurids and in the new sauropodiform (PVSJ 908) show a new pattern of pneumatization in an unprecedented region (i.e., prcdf) throughout the presacral region, and an improved pneumatic system among the cervico-dorsal transition that evolved well before the origin of Eusauropoda. Because only cervical and abdominal air sacs and lungs are able to produce pneumatic diverticula that invade the vertebrae, the presence of a sophisticated pneumatic complex on the neck of these taxa suggests that a cervical air sac system, and hence an improved avian-style respiratory system, were well developed already at the base of Sauropoda. 


\title{
REVISITING THE UPPER CRETACEOUS NOASAURID RECORD OF THE NEUQUÉN BASIN, PATAGONIA, ARGENTINA
}

\author{
M.A. Baiano ${ }^{1}$, R.A. Coria², G. Windholz ${ }^{1}$, And J.G. Meso ${ }^{1}$ \\ ${ }^{1}$ Instituto de Investigación en Paleobiología y Geología (IIPG)-Consejo Nacional de Investigaciones Científicas y Tecnológicas (CONICET), Uni- \\ versidad Nacional de Río Negro. Av. Roca 1242, R8332EXZ Gral. Roca, Río Negro,Argentina.mbaiano@unrn.edu.ar; gwindholz@unrn.edu.ar; \\ jgmeso@unrn.edu.ar \\ ${ }^{2}$ Consejo Nacional de Investigaciones Científicas y Tecnológicas (CONICET). Museo "Carmen Funes". Av. Córdoba 55, Q8318EBA Plaza Huin- \\ cul, Neuquén, Argentina.rcoria@unrn.edu.ar
}

Noasauridae is a family of abelisauroid theropods that has a scarce record in South America, which, up to now, was only represented by small-sized forms from the Hauterivian (Lower Cretaceous) Ligabueino andesi Bonaparte; the Santonian (Upper Cretaceous) Velocisaurus unicus Bonaparte; and the Campanian-Maastrichtian (Upper Cretaceous) Noasaurus leali Bonaparte and Powell. Here we report the recognition of noasaurid features in two medium-sized specimens, MCF-PVPH53 and 398, both consisting of proximal humeral ends. MCF-PVPH-53 was collected from the Coniacian-Turonian (Upper Cretaceous) levels of the Portezuelo Formation, which had been formerly assigned as an indeterminate abelisauroid that presents the noasaurid synapomorphy of a stout humeral head in proximal view, less than two times wider than long. In turn, MCF-PVPH-398, which comes from the Campanian Anacleto Formation, has a weakly developed greater tubercle positioned at the level of the internal tuberosity, resembling the condition present in the noasaurid Elaphrosaurus Janensch. Furthermore, the humeral head is inflated and the internal tuberosity is extremely reduced in both MCF-PVPH-53 and 398 , as in noasaurids like Elaphrosaurus and Masiakasaurus Sampson, Carrano and Forster, but unlike the ball-like humeral head and the well-developed internal tuberosity observed in Abelisauridae. The presence of noasaurids in the Turonian-Campanian interval enriches the South American record of Noasauridae, and depicts the presence of medium-sized noasaurids in the Upper Cretaceous, which, up to now, was composed of small-sized forms such as Masiakasaurus, Noasaurus and Velocisaurus.

\section{NEW ABELISAUROIDEA (THEROPODA, CERATOSAURIA) REMAINS FROM CERRO FORTALEZA (CERRO FORTALEZA FORMATION), SANTA CRUZ PROVINCE, ARGENTINA}

\author{
J.I. Canale ${ }^{1}$, A. Paulina-Carabajal ${ }^{2}$, A.H. Méndez ${ }^{3}$, and Y. Lee ${ }^{4 *}$ \\ 'Museo Paleontológico "Ernesto Bachmann". Centro Cívico s/n, 8311 Villa El Chocón, Neuquén, Argentina. \\ juanignaciocanale@hotmail.com \\ ${ }^{2}$ Instituto de Investigaciones en Biodiversidad y Medioambiente (INIBIOMA), Universidad Nacional del Comahue-Consejo Nacional de In- \\ vestigaciones Científicas y Tecnológicas (CONICET-UNCO). Quintral 1250, R8400FRF San Carlos de Bariloche, Argentina. \\ a.paulinacarabajal@conicet.gov.ar \\ ${ }^{3}$ Instituto Patagónico de Geología y Paleontología (IPGP), Centro Nacional Patagónico (CENPAT)-Consejo Nacional de Investigaciones Cien- \\ tíficas y Tecnológicas (CONICET). Boulevard Brown 2915, U9120ACD Puerto Madryn, Chubut, Argentina. arielhmendez@yahoo.com.ar \\ 4Seoul National University. Seoul, South Korea. ynlee@snu.ac.kr
}

Abelisauroid theropods constitute the most commonly recorded meat-eating dinosaurs from the Upper Cretaceous of Gondwana. In Argentina, they are known mainly from central and northern Patagonia, especially at the Neuquina basin. However, their record in the southern region of Patagonia is scarce, although the Cretaceous outcrops of the Magallanes basin are extensive. In this contribution we present new abelisauroid remains (MPM-PV 19111), consisting of two partial metatarsals, recovered from Cerro Fortaleza Formation (Campanian), Santa Cruz province. The metatarsal III (MPMPV 19111-1) is represented by a distal articular end, which shows a rectangular contour in distal view, and wide and welldeveloped collateral pits. The proximal end of the metatarsal IV (MPM-PV 19111-2) shows a D-shaped articular surface; with a gently concave medial surface for the articulation with the metatarsal III, a sharp posteromedial margin, and a flat lateral margin which curves medially at its dorsal sector, giving a triangular cross-section to the diaphysis; a combination of features present in abelisauroids. Other putative abelisauroid recovered from the same formation is the enigmatic Austrocheirus isasii, which phylogenetic relationships are yet unclear. Although both, Austrocheirus and the new specimen preserved part of the metatarsal III, which show similar dimensions, there are no clear morphologic traits that could suggest that both could represent the same taxon. The new specimen adds to the knowledge of the dinosaur fauna of Cerro Fortaleza Formation, supporting the presence of mid-sized abelisauroids in the Campanian of southern Patagonia, together with megaraptorid theropods, giant sauropod titanosaurs, and elasmarian ornithopods.

*Financial support by PICT 2015-0920 (to A.H.M.). 


\title{
¿QUÉ COMÍAN LOS GIGANTES? RESTOS VEGETALES ASOCIADOS A UN SAURÓPODO EN LA FORMACIÓN CAÑADÓN ASFALTO (JURÁSICO INFERIOR-MEDIO) CHUBUT, ARGENTINA
}

\author{
I. Davie ${ }^{1}$, I. Escapa ${ }^{1}$, D. POL ${ }^{1}$ Y J.L. CARBallido ${ }^{1}$ \\ ${ }^{1}$ Museo Paleontológico Egidio Feruglio. Av. Fontana 140, U9100GYO Trelew, Chubut, Argentina. \\ idavie@mef.org.ar; iescapa@mef.org.ar; dpol@mef.org.ar; jcarbadillo@mef.org.ar
}

Se recuperó una masa de sedimento enriquecido en materia orgánica, íntimamente asociada con restos articulados de un saurópodo, en las cercanías de la localidad de Cerro Cóndor, provincia del Chubut (Argentina). Estratigráficamente estos restos se ubican en la parte basal de la Formación Cañadón Asfalto, recientemente datada en el límite entre Jurásico Inferior y Jurásico Medio. La zona ventral del saurópodo permanecía expuesta y la cavidad del cuerpo quedó delineada por costillas alineadas. La masa oscura, ubicada dentro de la cavidad, poseía una forma tubular orientada dorsocaudalmente. El procesamiento de las muestras reveló una elevada concentración de fragmentos cuticulares, principalmente de hojas, y no se observaron restos orgánicos de otro tipo (e.g., madera, hueso, etc.). Los restos foliares fueron asignables a dos morfotipos, los cuales se encuentran asociados con ocasionales granos de polen de diversidad limitada. La buenas preservación de caracteres cuticulares permitió la asignación preliminar de los morfotipos foliares a las familias de coníferas Araucariaceae y Cheirolepidaceae, una asociación dominante en los bosques jurásicos de Gondwana. Los resultados sugieren firmemente que la masa consiste en el contenido del sistema digestivo del saurópodo; el cual, en forma previa a su enterramiento, se alimentó selectivamente con dos variedades de coníferas sin evidencia de otros componentes. Este hallazgo sostiene, en base a evidencia directa, las hipótesis previamente delineadas sobre la relación estrecha entre los saurópodos y las familias Araucariaceae y Cheirolepidaceae.

\section{NUEVO YACIMIENTO ICNOLÓGICO EN LA FORMACIÓN YACORAITE (MAASTRICHTIANO- DANIANO): QUEBRADA DE HUICHAIRA, JUJUY, ARGENTINA}

\author{
I. Díaz-Martínez ${ }^{1}$, P. Citton ${ }^{1}$, C. Cónsole-Gonella ${ }^{2}$, S. De Valais ${ }^{1}$ y P. Villafañe ${ }^{2 *}$ \\ ${ }^{1}$ Instituto de Investigación en Paleobiología y Geología (IIPG)-Consejo Nacional de Investigaciones Científicas y Tecnológicas (CONICET), \\ Universidad Nacional de Río Negro. Av. Roca 1242, R8332EXZ Gral. Roca, Río Negro, Argentina. idiaz@unrn.edu.ar; pcitton@unrn.edu.ar; \\ sdevalais@unrn.edu.ar \\ ${ }^{2}$ Instituto Superior de Correlación Geológica (INSUGEO), Universidad Nacional de Tucumán-Consejo Nacional de Investigaciones Científicas \\ y Tecnológicas (CONICET). Miguel Lillo 205, T4000JFE San Miguel de Tucumán, Tucumán, Argentina. carlosconsole@csnat.unt.edu.ar; \\ pgvillafane@csnat.unt.edu.ar
}

En los últimos años se han dado a conocer numerosas localidades con trazas fósiles cretácicas en el área de la Quebrada de Humahuaca (Provincia de Jujuy). Geológicamente, estas localidades corresponden a la Formación Yacoraite (Grupo Salta, Subgrupo Balbuena), unidad de edad Maastrichtiano-Daniano, que es parte de un sistema epicontinental con facies transicionales predominantes. En esta contribución se presenta un nuevo sitio icnológico en dicha área, ubicado en la Quebrada de Huichaira (S $23^{\circ} 34.422^{\prime}$; 0 65 $25.996^{\prime}$ ), donde se encontraron huellas de dinosaurio preservadas en una sucesión de calizas de diferentes texturas deposicionales, que preliminarmente se asignan a facies litorales. Las huellas se localizan en el tope de siete niveles estratigráficos, de los cuales seis están muy poco expuestos. En cada uno de estos últimos niveles son visibles únicamente una o dos huellas tridáctilas mal conservadas e impresas probablemente por dinosaurios terópodos. El afloramiento con mayor exposición cuenta con la presencia de al menos 20 huellas, de las cuales cinco son subredondeadas indeterminadas, y 15 son tridáctilas. Parte de ellas tienen el margen proximal bilobulado e impresiones de dígitos cortos y anchos con el borde distal redondeado. Se asignan al icnogénero Hadrosauropodus que típicamente es relacionado con dinosaurios hadrosáuridos. Las otras huellas de la superficie tienen improntas de los dígitos alargados y estrechos, y se relacionan con dinosaurios terópodos. Este hallazgo, junto a los realizados anteriormente en otras localidades de la Formación Yacoraite, confirma la alta capacidad de preservación de huellas de vertebrados fósiles que tienen los ambientes transicionales de esta unidad.

*Proyecto financiado por PI UNRN 40-A-402. 


\title{
A NEW SKELETON OF THE DINOSAUR PRECURSOR LEWISUCHUS ADMIXTUS ALLOWS REASSESSING THE TAXONOMY OF THE DINOSAURIFORMS FROM THE LOWER CARNIAN LEVELS OF THE CHAÑARES FORMATION (LA RIOJA PROVINCE)
}

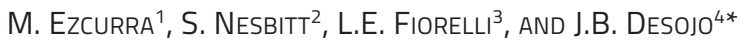 \\ 'Museo Argentino de Ciencias Naturales "Bernardino Rivadavia" (MACN)-Consejo Nacional de Investigaciones Científicas y Tecnológicas \\ (CONICET). Av. Ángel Gallardo 470, C1405DJR Ciudad Autónoma de Buenos Aires, Argentina. martindezcurra@yahoo.com.ar \\ 2Virginia Tech, Department of Geosciences. 24061, Blacksburg, Virginia, United States. sjn2104@vt.edu \\ ${ }^{3}$ Centro Regional de Investigaciones Científicas y Transferencia Tecnológica de La Rioja (CRILAR)-Consejo Nacional de Investigaciones \\ Científicas y Tecnológicas (CONICET). Entre Ríos y Mendoza s/n, 5301 Anillaco, La Rioja, Argentina. lucasfiorelli@gmail.com \\ ${ }^{4}$ División Paleontología Vertebrados, Museo de La Plata, Facultad de Ciencias Naturales y Museo, Universidad Nacional de La Plata. Paseo del \\ Bosque s/n, B1900FWA La Plata, Buenos Aires, Argentina.julideso2@gmail.com
}

The lower Carnian levels of the Chañares Formation (Ischigualasto-Villa Unión basin, La Rioja province) have yielded some of the most informative dinosaur precursor species known so far. However, these species are based on partial skeletons that in several cases hamper the comparison between them because of the absence of overlapping bones. The latter has generated a contentious debate about the synonym between two of these species, Lewisuchus admixtus and Pseudolagosuchus major. Here, we describe a new dinosauriform partial skeleton (CRILAR-Pv 552) recently collected in the Chañares Formation that for the first time preserves previously unknown anatomical regions and allows comparisons with other dinosauriform specimens. CRILAR-Pv 552 is referred to Lewisuchus admixtus because it possesses a proportionally large skull with recurved teeth ankylosed to the bone and lacks a coracoid foramen. The new specimen preserves a dorsally bowed dentary with a lateroventral shelf that is identical to a dentary associated with the holotype of Lewisuchus admixtus. The latter bone has been recently reinterpreted as probably belonging to a proterochampsid, but CRILAR-Pv 552 confirms that it instead is part of the holotype of the species. Additionally, the morphology of the new specimen is completely congruent with that of specimens of Pseudolagosuchus major, bolstering the hypothesis that the latter species is the same as Lewisuchus admixtus. We concluded that the holotype of Pseudolagosuchus major is not diagnostic at a species level, thus we propose that this species should be considered a nomen dubium, but its referred specimens can be referred to Lewisuchus admixtus.

*Financial support by Jurassic Foundation to MDE and PICT 2014-609 to JBD.

\section{NUEVOS MATERIALES DEL SAUROPODOMORFO BASAL ADEOPAPPOSAURUS MOGNAIMARTÍNEZ Y SUS IMPLICANCIAS EN EL ORIGEN DE UN SACRO "TIPO SAURÓPODO"}

\author{
G. Garcia ${ }^{1}$, R. Martínez ${ }^{1}$ y C. Apaldetti ${ }^{2}$ \\ IInstituto y Museo de Ciencias Naturales (IMCN), Universidad Nacional de San Juan. España 400 (norte), J5400DNQ San Juan, San Juan, Argentina. \\ gonzalogarciageo@gmail.com; martinez@unsj.edu.ar \\ ${ }^{2}$ Centro de Investigaciones de la Geósfera y la Biósfera (CIGEOBIO)-Consejo Nacional de Investigaciones Científicas y Tecnológicas (CONICET). \\ Av. Ignacio de la Roza 590, J5402DCS San Juan, San Juan, Argentina. capaldetti@unsj.edu.ar
}

La presencia de un sacro "tipo saurópodo" - 4 o más elementos sacrales- interpretado como respuesta al aumento del tamaño corporal, surge entre algunos Sauropodiformes y es característico de las formas más derivadas entre los Sauropodomorpha, es decir Sauropoda. En el presente trabajo damos a conocer un nuevo espécimen (PVSJ 896) de Adeopapposaurus mognai, asignado a este sobre la base de su procedencia y similitud general del esqueleto craneal y postcraneal. A partir del estudio anatómico del PVS 896 y la re-evaluación de los especímenes ya conocidos de A. mognai, se determinó que el sacro de esta especie está conformado por cuatro vértebras (DS+S1+S2+CS) y no por tres (DS+S1+S2) como se había considerado originalmente. El nuevo resultado filogenético recupera además a Xingxiulong chengi, un sauropodiforme con 4 vértebras sacras, en una posición menos derivada que la propuesta originalmente, formando una politomía con los massospondylidos y otros taxones no-sauropodiformes como Seitaad, Yunnanosaurus y Jingshanosaurus. Este resultado sugiere que la presencia de un sacro "tipo saurópodo" no es exclusiva de formas derivadas (e.g., Sauropodiformes) sino que surge también en formas basales no directamente emparentadas al origen de Sauropoda, ocurriendo independientemente más de una vez en la historia evolutiva del grupo. Estos resultados proporcionan nueva información acerca de las relaciones filogenéticas entre las formas no-Sauropoda de Sauropodomorpha y evidencian que la incorporación de vértebras sacras es una tendencia temprana en la historia evolutiva del grupo, no estando estrictamente relacionada al aumento del tamaño corporal en Sauropodomorpha. 


\title{
A POSSIBLE DICRAEOSAURID DENTAL PATTERN?
}

\author{
J.P. Garderes ${ }^{1}$, P.A. Gallina ${ }^{1}$, J.A. Whitlock², and N. Toledo ${ }^{3}$ \\ ${ }^{1}$ Fundación de Historia Natural Félix de Azara, Centro de Estudios Biomédicos, Biotecnológicos, Ambientales y Diagnóstico (CEBBAD)- \\ Universidad Maimónides. Hidalgo 775, C1405CBK Ciudad Autónoma de Buenos Aires, Argentina. jpgarderes@gmail.com; \\ pablo.gallina@fundacionazara.org.ar \\ 2Department of Science and Mathematics, Mount Aloysius College. 7373 Admiral Peary Hwy, 16630, Cresson, Pennsylvania, United States \\ of America.jwhitlock@mtaloy.edu \\ ${ }^{3}$ División Paleontología de Vertebrados, Unidades de Investigación Anexo Museo, Facultad de Ciencias Naturales y Museo, Universidad \\ Nacional de La Plata. Av. 122 y 60, B1904 La Plata, Buenos Aires, Argentina.ntoledo@fcnym.unlp.edu.ar
}

Cranial remains among Dicraeosauridae are scarce, even more considering remains with dental information (i.e., tooth bearing bones). Only Suuwassea Harris, Dicraeosaurus Janensch, and MMCh-PV 75 (from Bajada Colorada Formation, Neuquén, Argentina) preserved toothbearing cranial remains. Within Diplodocoidea, it was proposed that Dicraeosauridae had the condition of an equal functional teeth number between upper and lower jaws, based on Dicraeosaurus remains, which bears 16 alveoli in each jaw ( 4 in premaxilla, 12 in maxilla, and 16 in dentary). However, considering Suuwassea, the holotype and referred material stands against this proposal, because the upper jaw presents 11 alveoli (4 in premaxilla, 7 in maxilla) whereas the lower jaw bears 9 alveoli, here assumed to be complete in the alveolar count, despite its general incompleteness. MMCh-PV 75 supports the condition stated of an equal tooth number but with a lower count, showing 12 alveoli in each jaw (4 premaxillary and 8 maxillary teeth preserved as an isolated tooth row, and 12 teeth in articulation with the dentary). It has also been proposed an increase in the number of functional teeth within Dicraeosauridae. Suuwassea was recovered as a basal dicraeosaurid, while Dicraeosaurus being more derived. MMCh-PV 75 is recovered in a more derived position than Suuwassea, but more basal than Dicraeosaurus, thus this would support the incrementation in the number of dental elements along dicraeosaurid evolution.

\section{PRESERVATION OF AN ISOLATED TOOTH ROW IN A DICRAEOSAURID SAUROPOD DINOSAUR FROM PATAGONIA}

\author{
J.P. Garderes ${ }^{1}$, P.A. Gallina ${ }^{1}$, J.A. Whitlock ${ }^{2}$, and N. Toledo ${ }^{3}$ \\ ${ }^{1}$ Fundación de Historia Natural Félix de Azara, Centro de Estudios Biomédicos, Biotecnológicos, Ambientales y Diagnóstico (CEBBAD)- \\ Universidad Maimónides. Hidalgo 775, C1405CBK Ciudad Autónoma de Buenos Aires, Argentina. jpgarderes@gmail.com; \\ pablo.gallina@fundacionazara.org.ar \\ ${ }^{2}$ Department of Science and Mathematics, Mount Aloysius College. 7373 Admiral Peary Hwy, 16630, Cresson, Pennsylvania, United States \\ of America.jwhitlock@mtaloy.edu \\ ${ }^{3}$ División Paleontología Vertebrados, Unidades de Investigación Anexo Museo, Facultad de Ciencias Naturales yMuseo, Universidad Nacional \\ de La Plata. Av. 122 y 60, B1904 La Plata, Buenos Aires, Argentina.ntoledo@fcnym.unlp.edu.ar
}

Isolated tooth rows (ITR) are a kind of fossil preservation where a set of articulated teeth are conserved altogether with little or no surrounding bone structure associated. It has been proposed that these structures provide, along with a wrinkled pattern in the enamel surface of the tooth, an evidence for the presence of a keratinous beak among Sauropoda. Hitherto, several ITR were recorded within Eusauropoda, such as in the basal Shunosaurus, in the diplodocid Apatosaurus, in basal macronarian such as Europasaurus, Abydosaurus and Giraffatitan, and even in titanosaurians such as Phuwiangosaurus. Recently, the possible basal dicraeosaurid Lingwulong preserved an almost complete lower dentition as an isolated unit. The dicraeosaurid MMCh PV 75, from the Bajada Colorada Formation, Neuquén, preserves an almost fully complete upper dentition as an ITR (lacking of only the fourth right maxillary tooth and the eighth left maxillary tooth), found in close association with other bones of the skull. Only the left side shows a certain degree of tooth superposition with the first and second maxillary teeth over the fourth premaxillary tooth. It shows a "U"-shaped perimeter as common in other flagellicaudatans. However it is more caudally constrained than the lower jaw, possibly due to plastic deformation. Although further studies will try to assess the presence of a wrinkled pattern in the enamel in order to test the presence of a keratinous beak, the presence of an ITR in this taxon confirm this particular condition in dicraeosaurid sauropods as well as its wide distribution among Sauropoda. 


\title{
ESTUDIO PRELIMINAR DEL NEUROCRÁNEO DE UN NUEVO EUSAURÓPODO (DINOSAURIA: SAUROPODOMORPHA) DEL JURÁSICO TEMPRANO (FORMACIÓN CAÑADÓN ASFALTO), DE LA PROVINCIA DEL CHUBUT, ARGENTINA
}

\author{
K.L. Gomez ${ }^{1}$, A. Paulina-Carabajal ${ }^{2}$ y D. Pol ${ }^{1 *}$ \\ 1'Museo Paleontológico Egidio Feruglio. Fontana 140, U9100GYO Trelew, Chubut, Argentina. kgomez@mef.org.ar; dpol@mef.org.ar \\ 2Instituto de Investigaciones en Biodiversidad y Medioambiente (INIBIOMA), Universidad Nacional del Comahue-Consejo Nacional de Inves- \\ tigaciones Científicas y Tecnológicas (CONICET-UNCO). Quintral 1250, R8400FRF San Carlos de Bariloche, Argentina. \\ a.paulinacarabajal@conicet.gov.ar
}

El registro actual de los dinosaurios eusaurópodos del Jurásico Temprano y Medio es pobre a nivel mundial. El espécimen MPEF-PV 3301-1 colectado en la localidad de Cañadón Bagual (Formación Cañadón Asfalto, Chubut) confirmaría un origen del clado Eusauropoda mucho antes de lo que se creía (Toarciano), y es destacable que, además de material apendicular, se preservaron restos craneanos, entre ellos un neurocráneo prácticamente completo. Este se caracteriza por presentar un supraoccipital más ancho que alto como en Omeisaurus Young; un foramen magnum más alto que ancho como el presente en Camarasaurus Cope; procesos paraoccipitales orientados transversalmente, común en saurópodos; tubérculos basales no más anchos que el cóndilo, similar a Nebulasaurus Xing, Miyashita, Currie, You y Dong; una crista prootica bien desarrollada y de forma alar (= preotic pendant en terópodos), la cual se reduce hacia formas más derivadas; y procesos basipterigoideos cortos, como en Spinophorosaurus, Remes, Ortega, Fierro, Joger, Kosma y Ferrer. Una tomografía computada permitió la identificación de los forámenes neurovasculares, de los que se destacan un solo foramen para el NC XII, una rama oftálmica del NC V separada, y una posible fenestra epióptica. La presencia de una protuberancia ventral a la faceta proatlantal, y el borde ventral distal del proceso cultriforme cóncavo, son posibles autapomorfías para este nuevo taxón. La comparación de MPEF-PV 3301-1 con neurocráneos, principalmente de otros taxones filogenética y temporalmente cercanos, indica que posee tanto caracteres primitivos como derivados. La inclusión de estos caracteres en un análisis filogenético, permitirá entender la evolución de la neuroanatomía en el clado.

*Proyecto financiado por Agencia Nacional de Promoción Científica y Tecnológica PICT 2016-0481.

\section{OSTEOHISTOLOGÍA DE NEUQUENSAURUS POWELL, ASPECTOS PALEOBIOLÓGICOS INFERIDOS}

\author{
R. Gonzalez ${ }^{1}$ y A.I. CERda ${ }^{2 *}$
}

${ }^{1}$ Centro de Ecología Aplicada del Litoral (CECOAL)-Consejo Nacional de Investigaciones Científicas y Tecnológicas (CONICET) y Universidad Nacional del Nordeste. Ruta 5, km 2,5, 3400 Corrientes, Argentina. romigonzl95@gmail.com

2Instituto de Investigaciones en Paleobiología y Geología (IIPG)-Consejo Nacional de Investigaciones Científicas y Tecnológicas (CONICET), Universidad Nacional de Río Negro, Museo Provincial Carlos Ameghino. Belgrano 1700, Paraje Pichi Ruca (predio Marabunta), R8324CZH Cipolletti, Río Negro, Argentina. nachocerda6@gmail.com

Neuquensaurus es un titanosaurio acorazado cuyos restos provienen de afloramientos correspondientes a la Formación Anacleto (Santoniano-Campaniano), de la localidad rionegrina de Cinco Saltos. La presente contribución tiene como objetivo el de inferir, a partir de un estudio osteohistológico, aspectos vinculados a la dinámica de crecimiento de este dinosaurio. Asimismo, se busca ampliar el conocimiento en cuanto al grado de correlación temporal entre la maduración somática (momento en el que se alcanza la talla adulta) y cambios macroscópicos tales como la fusión de suturas neurocentrales ("maduración esqueletal"). Para ello, se estudiaron secciones delgadas obtenidas a partir de huesos apendiculares (fémur, húmero, tibia y fíbula) de los especimenes MCS-Pv-5 MCS-Pv-6; MCS-Pv-8; MCS-Pv-9; MLP-CS-1019. El tejido cortical exhibe un alto grado de remodelación secundaria, lo que imposibilita obtener información sobre el crecimiento en buena parte de la ontogenia. El tejido primario se encuentra relegado a la corteza externa. Se reconocen líneas de crecimiento detenido y annuli en el tejido primario, lo que indica un crecimiento discontinuo al menos durante etapas tardías del desarrollo. La presencia de una capa circunferencial externa en algunos ejemplares revela un crecimiento determinado. La ausencia de dicho tejido en el ejemplar MCS-Pv-5, el cual presenta una clara fusión de sus suturas neurocentrales de sus vértebras cervicales, dorsales y caudales, permite inferir que la maduración somática en Neuquensaurus era alcanzada con posterioridad a la maduración esqueletal.

*Proyecto subsidiado por PICT 2015-1021 y Jurassic Foundation. 


\title{
PHYLOGENETIC RELATIONSHIPS OF A NEW ALVAREZSAURID (DINOSAURIA: COELUROSAURIA) FROM THE ALLEN FORMATION (MAASTRICHTIAN, UPPER CRETACEOUS) OF RÍO NEGRO PROVINCE, ARGENTINA: IMPLICATIONS FOR THE ARGENTINIAN TAXA
}

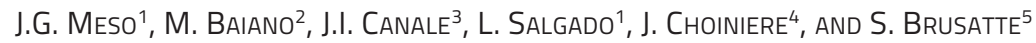 \\ ${ }^{1}$ Instituto de Investigación en Paleobiología y Geología (IIPG)-Consejo Nacional de Investigaciones Científicas y Tecnológicas (CONICET), \\ Universidad Nacional de Río Negro. Av. Roca 1242, R8332EXZ Gral. Roca, Río Negro, Argentina.jgmeso@unrn.edu.ar; Isalgado@unrn.edu.ar \\ ${ }_{2}^{2}$ Museo "Carmen Funes". Av. Córdoba 55, Q8318EBA Plaza Huincul, Neuquén, Argentina. mbaiano@unrn.edu.ar \\ ${ }^{3}$ Museo Paleontológico “Ernesto Bachmann". Villa El Chocón, 8311 Villa El Chocón, Neuquén, Argentina. jicanale@unrn.edu.ar \\ 4Department of Vertebrate Paleontology, American Museum of Natural History. Central Park West at 79th Street, NY 10024, Manhattan, \\ New York, USA. Jonah.Choiniere@wits.ac.za \\ ${ }^{5}$ School of GeoSciences, University of Edinburgh. Edinburgh 3FE, EH9, Edimburgo, Reino Unido, UK. sbrusatt@exseed.ed.ac.uk
}

Alvarezsauridae is a group of small-sized coelurosaurian theropods, known from Upper Cretaceous deposits of North America, Asia and South America. Although many new alvarezsaurid species have been recently discovered, and the knowledge of their anatomy has largely improved over the last decade, the phylogenetic relationships of Alvarezsauridae are still under debate. The specimen MPCN-PV 738 (previously catalogued as MGPIFD-GR 166/194 and MEPyG-177 by Salgado et al. and Coria et al. in previous works, respectively), from the Allen Formation, sheds some light on that issue. This specimen was included in a matrix of 888 characters and 163 avian and non-avian theropod taxa, and a phylogenetic analysis was performed using TNT. A strict consensus show MPCN-PV 738 nested in a small clade together with Bonapartenykus, with Patagonykus as sister group, all forming a clade recently recognized as Patagonykinae. The autapomorphies that distinguish this new alvarezsaurid are: ventral portion of coracoid strongly medially deflected (less than 120 degrees); anterior sector of transversal ridge on the coracoid robust and wide; grooves and striae only developed on the ventral surface of the coracoid, and dorsally limited by the transversal ridge; coracoid foramen ovoidal, with its major axis anteroventrally-posterodorsally oriented. The synapomorphies that support Patagonykinae are: transversal ridge on the lateral surface of coracoid; coracoid surface decorated with deep and narrow grooves and bumps; recess for the coracoid foramen and pubic apron greatly reduced, restricted to distal end of pubis. These results support previous ideas on the existence of an endemic clade of Patagonian alvarezsaurids.

\section{MODIFICATION AND REDUCTION OF THE FORELIMBS OF THE ALVAREZSAURIDS (THEROPODA, COELUROSAURIA)}

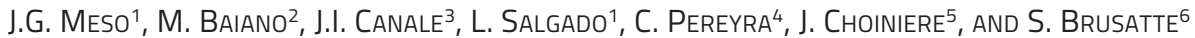

${ }^{1}$ Instituto de Investigación en Paleobiología y Geología (IIPG)-Consejo Nacional de Investigaciones Científicas y Tecnológicas (CONICET), Universidad Nacional de Río Negro. Av. Roca 1242, R8332EXZ Gral. Roca, Río Negro, Argentina.jgmeso@unrn.edu.ar; Isalgado@unrn.edu.ar 2Museo "Carmen Funes". Av. Córdoba 55, Q8318EBA Plaza Huincul, Neuquén, Argentina. mbaiano@unrn.edu.ar

${ }_{3}^{3}$ Museo Paleontológico "Ernesto Bachmann". Villa El Chocón, 8311 Villa El Chocón, Neuquén, Argentina. jicanale@unrn.edu.ar

${ }^{4}$ Facultad de Ciencias Naturales y Museo, Universidad Nacional de La Plata. Av. 120 y 60, 1900 La Plata, Buenos Aires, Argentina. cristian.pereyra.86cs@gmail.com

${ }^{5}$ Department of Vertebrate Paleontology, American Museum of Natural History. Central Park West at 79th Street, 10024, Manhattan, New York, USA. Jonah.Choiniere@wits.ac.za

${ }^{6}$ School of GeoSciences, University of Edinburgh. Edinburgh 3FE, EH9, Edimburgo, Reino Unido, UK. sbrusatt@exseed.ed.ac.uk

Alvarezsaurid forelimbs are markedly short and have a robust digit I ending in a stout ungual phalanx. Recently, two new basal alvarezsaurids, Bannykus and Xiyunykus Xu et al., were published, yielding evidence on the evolution of this part of the skeleton in this group of coelurosaurian theropod dinosaurs. The inclusion of Bannykus and Xiyunykus (both from the Lower Cretaceous of China), and the specimen MPCN-PV 738 (from the Upper Cretaceous of Patagonia), in a phylogenetic dataset of 888 characters and 163 avian and non-avian theropod taxa, shed some light on that issue. Our results not only show a better resolution of the internal relationships of Alvarezsauridae with respect to previous analyses; recognizing two different clades of derived alvarezsaurids from the Upper Cretaceous, one integrated by Asian forms, and other formed by Patagonian species, but also that the extreme modifications of the forelimb evolved gradually, leading to different anatomical configurations in both clades. The Patagonian forms have the proximal (preungual) phalanx longer than the ungual, while the derived forms from the Upper Cretaceous of Mongolia have a much shorter proximal phalanx compared with the ungual. The taxa from South American possess an ulnar olecranon process more robust and posteroventrally flexed, while in the forms from Asia, the olecranon is posteriorly projected. This last difference is also related with a variation in the articulation angle of the elbow in both subclades. In this way, these different trends in the forelimb modifications inside Alvarezsauridae may suggest different adaptations between Patagonian and Asian forms. 


\title{
THE NEUROANATOMY OF MEGARAPTOR NAMUNHUAIQUII (THEROPODA: MEGARAPTORIDAE) FROM THE UPPER CRETACEOUS OF PATAGONIA, A PRELIMINARY REPORT
}

\author{
A. Paulina-Carabajal ${ }^{1}$, And J.D. Porfiri ${ }^{2 *}$ \\ ${ }^{1}$ Instituto de Investigaciones en Biodiversidad y Medioambiente (INIBIOMA), Universidad Nacional del Comahue-Consejo Nacional de Inves- \\ tigaciones Científicas y Tecnológicas (CONICET-UNCO). Quintral 1250, R8400FRF San Carlos de Bariloche, Argentina. \\ a.paulinacarabajal@conicet.gov.ar \\ ${ }^{2}$ Museo de Ciencias Naturales de la Universidad Nacional del Comahue. Buenos Aires 1400, Q8300IBX Neuquén, Neuquén, Argentina. \\ jporfiri@gmail.com
}

Megaraptorid neuroanatomy has been studied in a single species with known braincase: Murusraptor barrosaensis Coria and Currie from the Upper Cretaceous of Patagonia. Here, we report the brain and inner ear of a juvenile specimen (MUCPV 595) of Megaraptor namunhuaiquii Novas, which were reconstructed using a microCT scan. The preserved braincase is small (approximately $6-7 \mathrm{~cm}$ long) and is incomplete, missing part of the skull roof, the basioccipital and all bones in front the prootics. The cranial endocast represents the hindbrain and part of the midbrain, including cranial nerves V, VII-XII and the caudal middle cerebral veins. The lack of laterosphenoids prevents to determine if the ophthalmic branch of the trigeminal nerve (V1) is separate or not. The inner ear is similar to other theropods, with an anterior semicircular canal (asc) larger than the posterior (psc), and the later larger than the lateral canal. The asc is not markedly larger than the psc (and is not posterodorsally expanded as in tyrannosaurids); they form an angle of approximately 85-90 degrees in dorsal view. The floccular process of the cerebellum is tubular and long, with its tip reaching the level of the psc. When compared to $M$. barrosaensis, the morphology of both, brain and inner ear are similar, showing short and ventrally convex medulla oblongata. M. namunhuaiquii however, has a markedly larger and longer floccular process. Further analysis of this data and comparisons with coelurosaur theropods will shed some light on the understanding of the phylogenetic relationships of this clade within Theropoda.

*Financial support by Agencia Nacional de Promoción Científica y Tecnológica PICT 2016-0481 (APC).

\section{REDIAGNOSIS OF RINCONSAURUS CAUDAMIRUS CALVO AND GONZÁLEZ RIGA (DINOSAURIA, SAUROPODA) FROM THE ANACLETO FORMATION (UPPER CRETACEOUS: CAMPANIAN) OF RINCÓN DE LOS SAUCES (NEUQUÉN, ARGENTINA)}

\author{
A. Pérez Moreno ${ }^{1,6}$, A. Otero ${ }^{2,6}$, J.L. Carballido ${ }^{1,6}$, L. Salgado 3,6 , J.O. Calvo ${ }^{4}$, And L.S. Filippi ${ }^{5}$ \\ 'Museo Paleontológico Egidio Feruglio. AV. Fontana 140, U9100GYO Trelew, Chubut, Argentina. aperezmoreno@mef.org.ar; \\ jcarballido@mef.org.ar \\ ²División Paleontología Vertebrados, Unidades de Investigación Anexo Museo, Facultad de Ciencias Naturales y Museo, Universidad Nacio- \\ nal de La Plata. Av. 122 y 60, B1904 La Plata, Buenos Aires, Argentina. alexandros.otero@gmail.com \\ ${ }^{3}$ Instituto de Investigación en Paleobiología y Geología (IIPG)-Consejo Nacional de Investigaciones Científicas y Tecnológicas (CONICET), \\ Universidad Nacional de Río Negro. Av. Roca 1242, R8332EXZ Gral. Roca, Río Negro, Argentina. Isalgado@unrn.edu.ar \\ ${ }^{4}$ Grupo de Transferencia Proyecto Dino. Ruta 51, km 65. Cátedras de Introducción a la Paleontología e Introducción a la Geología, Facultad de \\ Ingeniería, Universidad Nacional del Comahue. Buenos Aires 1400, Q8300IBX Neuquén, Neuquén, Argentina. jocalvo@hotmail.com \\ ${ }^{5}$ Museo Municipal Argentino Urquiza. Jujuy y Chaco s/n, Q8319BFA Rincón de los Sauces, Neuquén, Argentina. Isfilippi@gmail.com
}

Despite its great diversity, knowledge of many aspects of titanosaur sauropod anatomy is relatively poor, in part due to the fragmentary nature of many species. Rinconsaurus caudamirus is represented by several partial skeletons from a single locality from the Anacleto Formation (Upper Cretaceous, Campanian), in the north of Neuquén province, Argentina. Since its original description, this taxon has not been subjected to an exhaustive revision, which has raised a number of questions about its taxonomical status. Among them, stand out the fact that characters 1 and 2 from the three originally used for diagnosing it showed to be more widespread distributed among Titanosauria. Additionally, an important morphological variability is observed among caudal vertebrae, making necessary the revision of the assemblage originally stated out. Here we present a revision of the postcranial material of Rinconsaurus based on new observations and comparisons with related taxa. The observed morphological differences in caudal vertebrae could be well explained as the product of different ontogenetic stages and serial variations, whereas three new autapomorphic character were detected (1-posterior cervicals with the ventral end of the spinoprezygapohyseal lamina intercepting the intraprezygapophyseal lamina; 2-anteriormost caudals with two marked lateral faces with the lower face strongly constricted ventrally; 3-caudal vertebrae with the anterior articular surface markedly higher than the posterior). Therefore, based on the present study we sustain the monospecific interpretation of the assemblage and reinforce the status of Rinconsaurus caudamirus as a valid species. 


\title{
NUEVOS REGISTROS DE COELOPHYSOIDEA (DINOSAURIA; THEROPODA) Y SAUROPODOMORPHA (DINOSAURIA; SAURISCHIA) DE LA FORMACIÓN LOS COLORADOS (TRIÁSICO TARDÍO)
}

\author{
J.C. SOTOMAYOR ${ }^{1}$, J.M. LEARDI ${ }^{2}$, A. OteRO ${ }^{3}$ Y L.C. GAETANO ${ }^{1}$ \\ 'Departamento de Ciencias Geológicas, Facultad de Ciencias Exactas y Naturales, Instituto de Estudios Andinos "Don Pablo Groeber" (IDEAN), \\ Universidad de Buenos Aires-Consejo Nacional de Investigaciones Científicas y Tecnológicas (CONICET). Intendente Güiraldes 2160, Ciudad \\ Universitaria - Pabellón II, C1428EGA Ciudad Autónoma de Buenos Aires, Argentina. juancsotomayorch@gmail.com; Icgaetano@gl.fcen.uba.ar \\ 2Departamento de Biodiversidad y Biología Experimental, Facultad de Ciencias Exactas y Naturales, Universidad de Buenos Aires. Ciudad \\ Universitaria, Pabellón II, C1428EGA Ciudad Autónoma de Buenos Aires, Argentina.jmleardi@gl.fcen.uba.ar \\ ${ }^{3}$ División Paleontología de Vertebrados, Unidades de Investigación Anexo Museo, Facultad de Ciencias Naturales y Museo, Universidad \\ Nacional de La Plata. Av. 122 y 60, B1904 La Plata, Buenos Aires, Argentina. aleotero@fcnym.unlp.edu.ar
}

La Formación Los Colorados está compuesta por una sucesión de depósitos continentales correspondientes al Triásico Tardío, en la que se registra una importante fauna de tetrápodos, principalmente arcosaurios. En este trabajo se describen fragmentos fósiles colectados durante trabajos de campo en el Parque Nacional Talampaya (La Rioja, Argentina), que incluyen dos distales y una diáfisis de fémur, depositados en la UNLR. El fémur izquierdo (FI) presenta una fosa extensora poco profunda desplazada medialmente respecto al eje mayor y una cresta tibiofibular que se extiende sobrepasando posteriormente al cóndilo medial. Estas características se encuentran presentes exclusivamente entre los terópodos celofisoideos. Fl muestra diferencias anatómicas con otros restos femorales hallados del clado en la Formación (i.e., Powellvenator), y se encuentra en la misma escala de tamaño de Lucianovenator (3,5 cm de ancho transversal máximo), taxón del cual se desconoce su anatomía femoral. Por el otro lado, el fémur derecho (FD) muestra características más ampliamente distribuidas entre los dinosaurios saurisquios (e.g., cresta tibiofibular separada del cóndilo lateral por un surco) y similitudes generales con los sauropodomorfos (borde anterior plano, cóndilo medial subrectangular). Sin embargo, FD destaca por su pequeño tamaño, pudiendo tratarse de una forma juvenil o simplemente una forma pequeña. En consecuencia, Fl y FD, muestran diferencias en estructuras anatómicas y tamaño con los registros de celofísidos y sauropodomorfos de la Formación Los Colorados, por lo que aportan nueva información a la diversidad de los dinosaurios saurisquios.

\section{PALEOHISTOLOGÍA DE DOS DICRAEOSAURIDAE (DINOSAURIA, SAUROPODA) DEL CRETÁCICO INFERIOR DE PATAGONIA: IMPLICANCIAS PALEOBIOLÓGICAS Y METODOLÓGICAS}

\author{
G. WINDhOLZ ${ }^{1}$ y I. CERDA ${ }^{2 *}$
}

\begin{abstract}
${ }^{1}$ Instituto de Investigación en Paleobiología y Geología (IIPG)-Consejo Nacional de Investigaciones Científicas y Tecnológicas (CONICET), Universidad Nacional de Río Negro. Av. Roca 1242, R8332EXZ Gral. Roca, Río Negro, Argentina.gwindholz@unrn.edu.ar

${ }^{2}$ Instituto de Investigaciones en Paleobiología y Geología (IIPG)-Consejo Nacional de Investigaciones Científicas y Tecnológicas (CONICET), Universidad Nacional de Río Negro, Museo Provincial Carlos Ameghino. Belgrano 1700, Paraje Pichi Ruca (predio Marabunta), R8324CZH Cipolletti, Río Negro, Argentina. nachocerda6@gmail.com
\end{abstract}

La histología de la familia Dicraeosauridae hasta el momento está pobremente conocida, limitada a elementos apendiculares de Dicraeosaurus Janensch y Suuwassea Harris y Dodson. Damos a conocer la osteohistología del fémur, húmero y costilla dorsal de Amargasaurus Salgado y Bonaparte (MACN-N-15) y el fémur de Amargatitanis Apesteguía (MACN-PV-N53). El objetivo fue dilucidar el estadio ontogenético de los ejemplares y, en el caso de Amargasaurus, establecer cuál de los elementos representa una mejor opción para inferir edad mediante el conteo de líneas de crecimiento detenido [LCDs]. El hueso cortical de ambos ejemplares está fuertemente remodelado, con abundantes osteonas secundarias que alcanzan la periferia. El tejido primario es predominantemente de tipo entretejido y altamente vascularizado. El número mínimo de marcas de crecimiento en la costilla, el húmero y el fémur de Amargasaurus es de 7, 4 y 5 respectivamente, mientras que el fémur de Amargatitanis exhibe 13 LCDs. En ninguno de los elementos analizados se registró una capa circunferencial externa (CCE). Esto indicaría la ausencia de maduración somática para ambos ejemplares, aunque habrían alcanzado la madurez sexual por la alta densidad de LCDs en la periferia. Como resultado interpretamos que el ejemplar de Amargatitanis representaría un estadio ontogenético más avanzado que el de Armargasaurus, debido al alto grado de remodelación ósea y a una mayor cantidad de LCDs menos espaciadas. Dado que la costilla preserva más LCDs que los huesos largos, interpretamos que son buenos elementos para hacer estimaciones de edad, coincidiendo con estudios previos en macronarios basales.

*Proyecto financiado por PICT 2015-1021 a IAC. 


\title{
ESTRUCTURA POLICAMERADA EN VÉRTEBRAS CERVICALES DE VIAVENATOR EXXONI (ABELISAURIDAE; FURILEUSAURIA)
}

\author{
V. ZURRIAGUZ' ${ }^{1}$, L.S. FILIPPI² Y A.H. MÉNDEZ ${ }^{3}$
}

${ }^{1}$ Instituto de Investigación en Paleobiología y Geología (IIPG)-Consejo Nacional de Investigaciones Científicas y Tecnológicas (CONICET), Universidad Nacional de Río Negro. Av. Roca 1242, R8332EXZ Gral. Roca, Río Negro, Argentina.vzurriaguz@gmail.com

2Museo Municipal "Argentino Urquiza" Jujuy y Chaco S/n, Q8319BFA Rincón de los Sauces, Neuquén, Argentina. Isfilippi@gmail.com

${ }^{3}$ Instituto Patagónico de Geología y Paleontología (IPGP), Centro Nacional Patagónico (CENPAT)-Consejo Nacional de Investigaciones Científicas y Tecnológicas (CONICET). Boulevard Brown 2915, U9120ACD Puerto Madryn, Chubut, Argentina. arielhmendez@yahoo.com.ar

El registro fósil de los abelisáuridos es el más abundante entre los terópodos de Gondwana durante el Cretácico Superior. Uno de ellos es Viavenator exxoni, proveniente de la Formación Bajo de la Carpa (Santoniano), al norte de la provincia de Neuquén del cual se conocen en detalle numerosos restos óseos (e.g., neurocráneo y esqueleto axial). A pesar de esto, gran parte de su paleobiología es aún desconocida, especialmente en lo referente a su neumaticidad. En este trabajo presentamos una tomografía computada de una vértebra cervical anterior (cv5) de Viavenator, en la cual se ve una estructura policamerada tanto en el arco neural como en el centro vertebral. Conjuntamente, es posible observar un gran foramen neumático de posición ventral conectado al interior del centro vertebral. Este tipo de patrón de neumaticidad compuesto por múltiples cámaras fue descripto recientemente para noasáuridos, pero nunca fue estudiado en abelisáuridos, donde además el foramen neumático ventral es novedoso ya que no ha sido documentado previamente en otros terópodos y que posiblemente sea el correlato óseo de la presencia de un divertículo del saco aéreo cervical. La información aquí presentada podría estar indicando un rasgo compartido entre abelisáuridos y noasáuridos. Este tipo de estudios con tomografías computadas son importantes y muy valiosos, ya que la neumaticidad en terópodos en general solo se conoce a través de observación tanto de materiales fragmentados como de estructuras externas y poco de su estructura interna es conocido. 


\title{
SIMPOSIO \\ "TAFONOMÍA: EVALUANDO LA CALIDAD DEL REGISTRO FÓSIL"
}

\section{REDACIÓN DURÓFAGA EN GASTRÓPODOS DEL MIEMBRO LA TOSCA (FORMACIÓN HUITRÍN), CRETÁCICO INFERIOR DE LA CUENCA NEUQUINA, ARGENTINA}

\author{
C.S. Cataldo ${ }^{1}$, D.E. Fernández ${ }^{1}$ y L. LuCi ${ }^{1}$ \\ ${ }^{1}$ Instituto de Estudios Andinos "Don Pablo Groeber" (IDEAN), Facultad de Ciencias Exactas y Naturales, Universidad de Buenos Aires. Intendente \\ Güiraldes 2160, Ciudad Universitaria - 1º piso, C1428EGA Ciudad Autónoma de Buenos Aires, Argentina. \\ ceciliacataldo@gl.fcen.uba.ar; elizabeth@gl.fcen.uba.ar; leticialuci@gl.fcen.uba.ar
}

Sedimentitas del Miembro La Tosca de la Formación Huitrín en dos localidades del sur de Mendoza preservan el primer caso documentado de predación por durofagia sobre conchillas de gastrópodos del Cretácico Inferior de la Cuenca Neuquina. El caso de estudio involucra dos conjuntos de conchillas de una misma especie, Paleoanculosa macrochilinoides (Doello-Jurado) (Acteonoidea), colectadas en Quebrada del Gastrópodo y Ranquil 1 en un espeso nivel de margas correlacionable entre las dos localidades e interpretadas como depósitos de lagoon. El número total de ejemplares es superior a 1.000. Ambos conjuntos de muestras fueron examinados en busca de cicatrices de predación subletales, reconocidas a partir de la reparación ulterior de la conchilla. Se identificaron ejemplares con entre una y tres cicatrices, las cuales se caracterizan por presentarse principalmente en la última vuelta pero también en vueltas de la espira. Morfológicamente, las cicatrices se clasifican como márgenes crenulados y se caracterizan por tener poca profundidad, ser relativamente suaves y presentar pocas mellas agudas distribuidas en forma irregular. A partir de la muestra estudiada se calcularon las frecuencias de reparación que se utilizan como indicadores de predación por trituración. Asimismo, se analizó la frecuencia de predación por intervalos de tamaño, a fin de evaluar la efectividad de conchillas de distinto tamaño en cuanto a su función antipredatoria. El origen de las cicatrices se atribuye, tentativamente, a predación por crustáceos decápodos, de los cuales si bien no hay registros corpóreos en el Miembro La Tosca sí los hay en la infrayacente Formación Agrio.

\section{ANÁLISIS TAFONÓMICO DE UN MISTICETO ARTICULADO DEL MIOCENO TARDÍO DE PENÍNSULA VALDÉS (CHUBUT, ARGENTINA)}

\author{
N.D. FARRON11,3, D. LAZO², J.I. CUITIÑO ${ }^{3}$ Y M. BUONO ${ }^{3 *}$ \\ ${ }^{1}$ Departamento de Ciencias Geológicas, Facultad de Ciencias Exactas y Naturales, Universidad de Buenos Aires. Ciudad Universitaria, Pabe- \\ Ilón 2, $1^{\circ}$ Piso, C1428EGA Ciudad Autónoma de Buenos Aires, Buenos Aires, Argentina.ndfarroni@gmail.com \\ 2Departamento de Ciencias Geológicas, Facultad de Ciencias Exactas y Naturales, Instituto de Estudios Andinos "Don Pablo Groeber" (IDEAN), \\ Universidad de Buenos Aires-Consejo Nacional de Investigaciones Científicas y Tecnológicas (CONICET). Intendente Güiraldes 2160, Ciudad \\ Universitaria - Pabellón II, C1428EGA Ciudad Autónoma de Buenos Aires, Argentina.dlazo@gl.fcen.uba.ar \\ ${ }^{3}$ Instituto Patagónico de Geología y Paleontología (IPGP), Centro Nacional Patagónico (CENPAT)-Consejo Nacional de Investigaciones Cien- \\ tíficas y Tecnológicas (CONICET). Boulevard Brown 2915, U9120ACD Puerto Madryn, Chubut, Argentina. jcuitino@cenpat-conicet.gob.ar; \\ buono@cenpatconicet.gob.ar
}

En la actualidad, en Argentina no existen antecedentes sobre estudios tafonómicos de cetáceos fósiles. Trabajos de prospección en el NE de Chubut resultaron en el hallazgo de un misticeto preservado en la parte inferior de la Formación Puerto Madryn (Mioceno Tardío) en el área de Punta Buenos Aires, Península Valdés. Este representa el primer registro de un esqueleto articulado para este grupo en Argentina. El objetivo de este trabajo es determinar los procesos pre y post enterramiento que propiciaron esta preservación excepcional. Para ello se realizó un estudio tafonómico con un enfoque multidisciplinario, que incluye: (1) determinación taxonómica del ejemplar y estudio de sus características ecológicas; (2) 
relevamiento sedimentológico, estratigráfico y paleontológico de las capas portadoras; (3) relevamiento tafonómico detaIlado; (4) análisis icnológico; e (5) integración de datos paleoambientales. Los resultados preliminares indican que el ejemplar corresponde a un Balaenidae, que murió y se depositó sobre el lecho marino en posición ventral. El alto grado de articulación y asociación de los elementos óseos, y la moderada fragmentación de estos, indican un transporte hidráulico bajo sobre el fondo. Además, los atributos tafonómicos (e.g., articulación, fragmentación, orientación) determinados en los invertebrados fósiles asociados y los icnogéneros registrados, refuerzan esta interpretación. Toda la información relevada permite inferir un ambiente de plataforma, de baja energía y oxigenación normal, con una tasa de sedimentación acorde a un enterramiento relativamente rápido del ejemplar.

*Proyecto financiado por PICT 20150792.

\title{
ESTUDIOS TAFONÓMICOS Y PALEOECOLÓGICOS DE LOS CONJUNTOS DE MAMÍFEROS DE AMÉRICA DEL SUR AUSTRAL DURANTE LOS EVENTOS CÁLIDOS DEL HOLOCENO
}

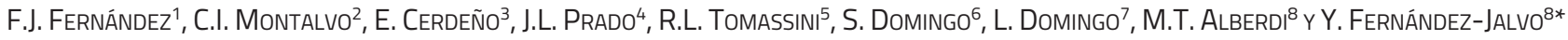 \\ ${ }^{1}$ Consejo Nacional de Investigaciones Científicas y Tecnológicas (CONICET). Cátedra de Anatomía Comparada, Facultad de Ciencias Natu- \\ rales y Museo, Universidad Nacional de La Plata. Calle 64 s/n (entre diag. 113 y calle 120), B1904DZA La Plata, Buenos Aires, Argentina. \\ fernandezf77@yahoo.com.ar \\ ${ }^{2}$ Facultad de Ciencias Exactas y Naturales, Universidad Nacional de La Pampa, Uruguay 151, L6300CLB Santa Rosa, La Pampa, Argentina. \\ cmontalvo@exactas.unlpam.edu.ar \\ ${ }^{3}$ Paleontología, Instituto Argentino de Nivología, Glaciología y Ciencias Ambientales (IANIGLA), Centro Científico Tecnológico Mendoza- \\ Consejo Nacional de Investigaciones Científicas y Tecnológicas (CONICET). Avenida Ruiz Leal s/n, 5500 Mendoza, Mendoza, Argentina. \\ espe@mendoza-conicet.gob.ar \\ ${ }^{4}$ Instituto de Investigaciones Arqueológicas y Paleontológicas del Cuaternario Pampeano (INCUAPA)-Departamento de Arqueología, Uni- \\ versidad Nacional del Centro. Av. Del Valle 5737, B7400JWI Olavarría, Buenos Aires, Argentina. jprado@soc.unicen.edu.ar \\ Instituto Geológico del Sur (INGEOSUR)-Departamento de Geología, Universidad Nacional del Sur (UNS)-Consejo Nacional de Investigacio- \\ nes Científicas y Tecnológicas (CONICET). Av. Alem 1253, B8000CPB Bahía Blanca, Buenos Aires, Argentina. rodrigo.tomassini@yahoo.com.ar \\ ${ }^{6}$ Departamento de Ecología Evolutiva, Estación Biológica de Doñana (CSIC). Américo Vespucio 26, 41092 Sevilla, Sevilla, España. \\ soldomingo@gmail.com \\ ${ }^{7}$ Departamento de Geodinámica, Estratigrafía y Paleontología Facultad de Ciencias Geológicas, Universidad Complutense de Madrid. C/José \\ Antonio Novais 12, 28040 Madrid, Madrid, España. Idomingo@ucm.es \\ ${ }^{8}$ Departamento de Paleobiología, Museo Nacional de Ciencias Naturales (CSIC). José Gutiérrez Abascal 2, 28006 Madrid, Madrid, España. \\ malberdi@mncn.csic.es; yfj@mncn.csic.es
}

Se evalúan las asociaciones faunísticas de mamíferos del Holoceno de América austral, considerando sus requerimientos ecológicos. En este contexto, la combinación de estudios taxonómicos y tafonómicos permiten valorar las características de los sistemas bióticos y abióticos, a través de la información de los conjuntos de mamíferos registrados durante la época evaluada. Este tipo de análisis facilita la comparación al máximo detalle de la diversidad, así como las fluctuaciones de los cambios ocurridos por calentamiento global en asociaciones faunísticas del sur de Brasil, Chile central y centro-oeste, Pampa y Patagonia en Argentina. Los períodos de interés incluyen el Óptimo Climático del Holoceno ( 8,2 ka AP) y un evento árido de carácter global aún no descrito en América del Sur (4,2 ka AP), con repercusión en migraciones humanas y civilizaciones de la Mesopotamia del Próximo Oriente. Con esos objetivos, se conformó una base de datos taxonómicos y tafonómicos de las asociaciones de mamíferos fósiles de la región. Los taxones presentes se agruparon por masa corporal estimada (megamamíferos > 1 tonelada; muy grandes $180-1000 \mathrm{~kg}$; grandes $44-180 \mathrm{~kg}$; medianos $5-44 \mathrm{~kg}$; pequeños $<5 \mathrm{~kg}$ ) y por tipo de dieta (hipercarnívoro, carnívoro, insectívoro, omnivoro, herbívoro y sus variantes). La información resultante proporciona un punto de referencia útil para reconocer una tasa "normal" o "anormal" de cambio faunístico que se pueda vincular con el cambio climático. Finalmente, proporciona elementos comparativos para analizar cambios faunísticos actuales que podrían reflejar una respuesta única al calentamiento global.

*Proyecto financiado por i-COOP 2017B-20287; CGL 2016-79334-P. 


\title{
ENSAMBLES DE MUERTE DE OSTRÁCODOS A LO LARGO DE UN GRADIENTE DE SALINIDAD EN LA LAGUNA COSTERA MAR CHIQUITA (PROVINCIA DE BUENOS AIRES, ARGENTINA)
}

\author{
F.L. Flores ${ }^{1,2}$, L. FerRero $0^{1,2}$ y C.G. De Francesco ${ }^{1 *}$ \\ ${ }^{1}$ Instituto de Investigaciones Marinas y Costeras (IIMyC), Facultad de Ciencias Exactas y Naturales, Universidad Nacional de Mar del Plata \\ (UNMdP). Rodriguez Peña 4046, B7602GSD Mar del Plata, Buenos Aires, Argentina. floresfabiolucas@gmail.com; marmart@mdp.edu.ar; \\ cgdefra@mdp.edu.ar \\ ${ }^{2}$ Instituto de Geología de Costas y del Cuaternario (IGCyC), Facultad de Ciencias Exactas y Naturales, Universidad Nacional de Mar del Plata \\ (UNMdP)-Comisión de Investigaciones Científicas (CIC). Dean Funes 3350, B7602AYL Mar del Plata, Buenos Aires, Argentina.
}

Con el objetivo de obtener información actualista útil para la reconstrucción de ambientes estuáricos holocenos, se analizó la diversidad de ostrácodos en ensambles de muerte (EM) depositados a lo largo de un gradiente de salinidad en la laguna

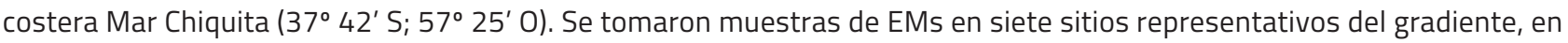
verano e invierno (2017-2018). Se calcularon índices de riqueza específica, diversidad de Shannon y equitatividad. Los índices mostraron diferencias entre sitios. Los tres más cercanos a la desembocadura presentaron valores más altos que los restantes, registrando un mayor número de especies eurihalinas y euhalinas. Esto podría deberse a la disponibilidad de un mayor número de nichos ecológicos y/o condiciones tróficas favorables en las proximidades de la desembocadura (e.g., variaciones de salinidad y condiciones hidrodinámicas) o representar ensambles de mezcla originados por procesos tafonómicos, en una zona de mayor energía ambiental. Las especies dominantes en todos los sitios fueron Cyprideis salebrosa hartmanni, Callistocythere aspereticulata y Cytherura dimorphica. La coexistencia de estos taxa podría deberse a su preferencia por aguas cloruradas sódicas, con amplias variaciones de salinidad debido a las influencias de mareas y fluctuaciones en los aportes de aguas continentales. La mayoría de los ensambles presentó una elevada proporción de individuos juveniles. Los resultados aquí obtenidos son preliminares y serán complementados con estudios de fidelidad entre las biocenosis y las tanatocenosis, así como estudios tafonómicos en diferentes condiciones ambientales, que se encuentran actualmente en desarrollo.

*Proyecto financiado por Exa 775.

\section{ANALYSIS OF SUBSTRATE VARIATIONS RELATED TO DINOSAUR TRACK PRESERVATION FROM THE AGUADA DE TUCO AREA, CANDELEROS FORMATION (CENOMANIAN), NEUQUÉN BASIN, ARGENTINA}

\author{
M. Heredia ${ }^{1}$, P.J. Pazos ${ }^{1}$, and I. Díaz Martínez ${ }^{2 *}$ \\ 1 Instituto de Estudios Andinos "Don Pablo Groeber" (IDEAN), Universidad de Buenos Aires. Intendente Güiraldes 2160, C1428EGA Ciudad \\ Autónoma de Buenos Aires, Argentina.heredia@gl.fcen.uba.ar; pazos@gl.fcen.uba.ar \\ 2 Instituto de Investigaciones en Paleobiología y Geología (IIPG)-Consejo Nacional de Investigaciones Científicas y Tecnológicas (CONICET), \\ Universidad Nacional de Río Negro. Av. Roca 1242, R8332EXZ General Roca, Río Negro, Argentina. idiaz@unrn.edu.ar
}

Fossil tracks provide direct information of the activity of a trackmaker during the interaction with the substrate. Track morphology can exhibit differences as a response to changes in substrate physical parameters like humidity, grain-size and sediment composition, as well as response to the limb dynamics and foot anatomy of the trackmaker. Recently, several dinosaur tracks with different preservational variants were found in a tracksite under study of the Candeleros Formation (Aguada de Tuco). An unusual narrow-gauge sauropod trackway preserved in fine-grained sandstones from floodplain deposits was documented. Each left track shows bulky and high (vertical) rims, significantly larger than the right ones. Thus a different substrate property between them is envisaged. These bulky rims also show well-preserved and non-deformed ripples on the top, suggesting a moist underlying substrate but with a dried or stabilized top that was only displaced upward during the track formation. The same stratigraphic surface contains a set of three extended tridactyl trackways, deeply impressed with no variation in the morphology of each track. In consequence no changes in substrate properties are interpreted between them. However, some discontinuous and shallow sauropod tracks were produced before the tridactyl trackways as they are overimprinted. Interestingly, $10 \mathrm{~m}$ apart a cluster of theropod trackways exhibit larger and shallower tracks than the previous one, which indicate a more consistent substrate, considering that they correspond to larger producers in the last case. In conclusion, the general track morphology is mainly conditioned by changes in substrate consistency over time in the same area.

${ }^{*}$ Financial support by PIP CONICET (2015-2017) and UBACyT Grant (2016-2018) to Pablo J. Pazos. This is contribution C-152 of the Instituto de Estudios Andinos Don Pablo Groeber. 


\title{
DIFERENCIACIÓN QUÍMICA EN LA PRESERVACIÓN DE CAPARAZONES DE ESPINICAUDADOS JURÁSICOS MEDIANTE ESPECTROMETRÍA DE ABLACIÓN LÁSER
}

\author{
V.C. Jiménez ${ }^{1}$, M.D. Monferran ${ }^{1}$, R.G. Pellerano² ${ }^{2}$ E.R. Pereira-Filhob ${ }^{3}$ y O.F. Gallego ${ }^{1 *}$
}

${ }^{1}$ Centro de Ecología Aplicada del Litoral (CECOAL)-Consejo Nacional de Investigaciones Científicas y Tecnológicas (CONICET). Ruta 5, Km 2.5, 3400 Corrientes, Corrientes, Argentina.victoriajimenez70@yahoo.com.ar; monfdm@gmail.com; ofgallego@live.com.ar

2Instituto de Química Básica y Aplicada del Nordeste Argentino (IQUIBA-NEA)-Consejo Nacional de Investigaciones Científicas y Técnicas (CONICET)-Universidad Nacional del Nordeste (UNNE). Av. Libertad 5470, W3404AAS Corrientes, Corrientes, Argentina. gpellera@gmail.com ${ }^{3}$ Universidad Federal de São Carlos, Departamento de Química, Grupo de Análisis Instrumental Aplicada. Rodovia Washington Luis s/n, km 235, 676 São Paulo, São Carlos, Brasil.erpf@ufscar.br

Los espinicaudados son un grupo de pequeños crustáceos bivalvos de ambientes continentales empleados generalmente como herramientas bioestratigráficas e indicadores paleoambientales. Estos organismos presentan un caparazón quitinoso y biomineralizado, el cual constituye normalmente el único elemento preservado en el registro fósil. El propósito de este trabajo es identificar diferencias químicas en la preservación de los caparazones de espinicaudados fósiles de la Formación La Matilde (Jurásico Medio). Para esto, se realizaron análisis químicos empleando la técnica de espectrometría de plasma inducido por ablación láser (LIBS) en áreas puntuales en los caparazones de Eosolimnadiopsis santacrucensis? Gallego y en sus rocas portadoras provenientes de las localidades Puesto Raspuzzi (PR) y Mina del Gobierno (MdG) en el Gran Bajo de San Julián (Santa Cruz). Los resultados obtenidos del análisis del caparazón en PR indican que está compuesto principalmente de calcio, magnesio y sodio, con picos menores de silicio. Esta composición difiere de la composición elemental en caparazones de MdG, donde el silicio es abundante y el calcio relativamente insignificante, esto fue observado también en las rocas. Estas diferencias químicas entre los caparazones están reflejadas en sus modos de preservación, en el cual, los caparazones en PR se encuentran mineralizados exhibiendo una coloración oscura diferente a la roca. Por otro lado, en MdG los caparazones corresponderían a moldes externos con similar coloración a la roca que los contiene.

*Proyecto subsidiado por PIP-CONICET 11220150100117 (responsable Dra. Nora Cabaleri).

\section{NUEVO REGISTRO DE OSTRÁCODOS CONTINENTALES DE LA FORMACIÓN PLOTTIER, GRUPO NEUQUÉN, PROVINCIA DE NEUQUÉN}

\begin{abstract}
R.G. KIHN ${ }^{1}$ Y J. CALVO ${ }^{2 *}$
${ }^{1}$ Instituto de Ciencias de la Tierra y Ambientales de La Pampa (INCITAP)-Consejo Nacional de Investigaciones Científicas y Técnicas (CONICET); Facultad de Ciencias Exactas y Naturales, Universidad Nacional de La Pampa. Mendoza 109, L6302EPA Santa Rosa, La Pampa, Argentina. rgkihn@gmail.com

${ }^{2}$ Grupo de Transferencia Proyecto Dino. Ruta 51, km 65. Cátedras de Introducción a la Paleontología e Introducción a la Geología, Facultad de Ingeniería, Universidad Nacional del Comahue. Buenos Aires 1400, Q8300IBX Neuquén, Neuquén, Argentina. jocalvo@hotmail.com

En este trabajo se estudiaron muestras de sedimento proveniente del yacimiento Babilonia, Parque Natural Geo-Paleontológico Proyecto Dino ubicado en la costa norte del Lago Los Barreales, a 90 km de la ciudad de Neuquén. Los niveles portadores corresponden a pelitas y areniscas muy finas de color verde, base de la Formación Plottier, Grupo Neuquén. Babilonia ha dado numerosos restos fósiles que incluyen vértebras, mandíbulas y dientes de dinosaurios terópodos y saurópodos titanosáuridos, dientes de cocodrilos, rizoconcreciones y numerosas raíces en perfecto estado. Entre los invertebrados hasta el momento solo se había mencionado la presencia de pelecípodos asignados al género Diplodon sp. Técnicas de disgregación de rocas permitieron descubrir numerosos ejemplares de Ostracoda con un buen estado de preservación. Los mismos corresponden a las familias Ilyocyprididae y Limnocytheridae, la primera está representada por cuatro especies: Neuquenocypris calfucurensis? Musacchio, Neocyprideis zampalensis Angelozzi, Neuquenocypris tenuipunctata Musacchio y Simeoni y Neuquenocypris nahuelniyuensis Musacchio; mientras que Limnocytheridae está representada solo por Vecticypris sp. La asociación microfaunística y los moluscos hallados permiten inferir un ambiente depositacional correspondiente a un cuerpo de agua dulce a oligohalino somero y de baja energía para los niveles estudiados de los tramos inferiores de la Formación Plottier en este sector de la Cuenca Neuquina; esta información permite además corroborar la hipótesis propuesta con anterioridad por otros autores sobre la presencia, en algunos sectores, de meandros abandonados.
\end{abstract}

*Proyecto financiado por Universidad Nacional Comahue 04-I-231/2017; Chevron S.A. Gobierno Provincia Neuquén y Fundación Luciérnaga. 


\title{
ANÁLISIS TAFONÓMICO PRELIMINAR DE UNA ASOCIACIÓN DE OSTRAS REGISTRADAS SOBRE UNA PALEOSUPERFICIE CERCANA AL TOPE DE LA FORMACIÓN CHACHAO, LOCALIDAD CUESTA DEL CHIHUIDO, MENDOZA
}

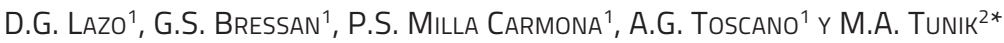 \\ 'Departamento de Ciencias Geológicas, Facultad de Ciencias Exactas y Naturales, Instituto de Estudios Andinos "Don Pablo Groeber" (IDEAN), \\ Universidad de Buenos Aires-Consejo Nacional de Investigaciones Científicas y Tecnológicas (CONICET). Intendente Güiraldes 2160, Ciu- \\ dad Universitaria - Pabellón II, C1428EGA Ciudad Autónoma de Buenos Aires, Argentina.dlazo@gl.fcen.uba.ar; gbressan@gl.fcen.uba.ar; \\ millacarmona@gl.fcen.uba.ar; atoscano@gl.fcen.uba.ar \\ 2Instituto de Investigación en Paleobiología y Geología (IIPG)-Consejo Nacional de Investigaciones Científicas y Tecnológicas (CONICET), Uni- \\ versidad Nacional de Río Negro. Av. Roca 1242, R8332EXZ Gral. Roca, Río Negro, Argentina. mtunik@unrn.edu.ar
}

Se presentan resultados de un análisis tafonómico realizado sobre ostras del género Aetostreon Bayle registradas sobre una paleosuperficie expuesta en el perfil Cuesta del Chihuido de la Formación Chacho en Mendoza. Dicha superficie se ubica estratigráficamente justo por debajo de la base de la suprayacente Formación Agrio. Las otras se disponen en el tope de un banco tabular de matriz micrítica de color gris y $40 \mathrm{~cm}$ de espesor que presenta empaquetamiento disperso a suelto en algunos sectores (floatstone). Se relevaron un total de 26 cuadrantes de $1 \mathrm{~m}^{2}$ distribuidos sobre una transecta NW-SE de $2.765 \mathrm{~m}$ de largo que incluyeron 838 especímenes de ostras. Teniendo en cuenta los datos en su conjunto se registró que el 60,4\% estaba articulado contra un $29,5 \%$ desarticulado, solo un $12,8 \%$ presentó algún tipo de bioerosión y un $64,5 \%$ de las articuladas se encontraban en orientación transversal "de canto" y un 27,1\% "convexo hacia abajo". El análisis exploratorio de mínimos cuadrados parciales (PLS) indica que la bioerosión y la abundancia de conchillas "convexo hacia arriba" son las variables con mayor influencia sobre la correlación entre el set de variables tafonómicas y la distancia a la línea de costa, disminuyendo ambas costa afuera. Este conjunto de datos tafonómicos por un lado refuerza hipótesis paleoambientales previas que sostenían sobre la base de análisis de facies que el ambiente de depositación corresponde a depósitos submareales de baja energía de una rampa carbonática, y por otro lado indica que la asociación de ostras representa un time-averaging bajo a moderado.

*Proyecto financiado por PICT 2015-1381.

\section{LA TAFONOMÍA COMO EXPERIENCIA EDUCATIVA: UN CASO PRÁCTICO}

\author{
L. López ${ }^{1}$, F. Colileo ${ }^{1}$, D. Pino² ${ }^{2}$ A. Navarrete ${ }^{1}$, S. Garcia ${ }^{1}$, P. Paniceres ${ }^{3}$ y I. Díaz Martínez ${ }^{2}$
}

${ }^{1}$ Universidad Nacional de Río Negro, Sede Alto Valle-Valle Medio. Estados Unidos 750, R8332BRJ General Roca, Río Negro, Argentina. lisandro.lopez33@yahoo.com.ar; nanda1310@hotmail.com; anavarrete@unrn.edu.ar; sgarcia@unrn.edu.ar

2Instituto de Investigación en Paleobiología y Geología (IIPG)-Consejo Nacional de Investigaciones Científicas y Tecnológicas (CONICET), Universidad Nacional de Río Negro. Av. Roca 1242, R8332EXZ Gral. Roca, Río Negro, Argentina.dapino@unrn.edu.ar; inaportu@hotmail.com ${ }_{3}^{3}$ Municipalidad de General Roca. Bartolomé Mitre 710, R8332HLP General Roca, Río Negro, Argentina.pablojpaniceres@gmail.com

El actualismo, entendido como "el presente es la clave del pasado", tiene una gran tradición en paleontología, y su vertiente experimental ha sido muy utilizada en varias de sus disciplinas. La tafonomía actualista o actuotafonomía utiliza las formas y procesos actuales para ayudar al estudio de modelos de preservación en el registro fósil. En este contexto, y como experiencia educativa dentro de la cursada de la asignatura de Tafonomía y Paleoecología perteneciente al quinto año de la Licenciatura de Paleontología de la Universidad Nacional de Río Negro, se analizaron los procesos de descomposición bioestratinómica y fosildiagenética temprana en dos ejemplares de Gallus gallus (Linnaeus) de comercio. El ejemplar 1 fue enterrado a $40 \mathrm{~cm}$ de profundidad y el ejemplar 2 fue colocado a la intemperie, ambos en un paraje cercano a General Roca (Área Natural Protegida Paso Córdoba) y protegidos por mallas metálicas para evitar la acción del carroñeo de grandes vertebrados. En visitas periódicas al lugar, se identificaron varios parámetros como los tipos de insectos presentes y la apariencia (color, olor, forma, etc.) del ejemplar 2. Después de cinco meses del inicio de la actividad, se desenterró el ejemplar 1 observando que se encontraba en un estado similar al inicial, muy diferente al ejemplar 2 que solo preserva piel seca y huesos. Como cierre de la experiencia, se analizó la relación existente entre el enterramiento rápido/exposición subaérea y la calidad de preservación de los ejemplares estudiados, y se discutió como se representarían ambos casos en el registro fósil. 


\title{
ANÁLISIS PRELIMINAR DE LA FAUNA INCRUSTANTE EN GASTRÓPODOS DEL MIEMBRO PILMATUÉ (FORMACIÓN AGRIO), CRETÁCICO INFERIOR DE LA CUENCA NEUQUINA
}

\author{
L. LUCI ${ }^{1}$ Y C.S. CATALDO ${ }^{1}$
}

${ }^{1}$ Instituto de Estudios Andinos "Don Pablo Groeber" (IDEAN), Universidad de Buenos Aires-Consejo Nacional de Investigaciones Científicas y Tecnológicas (CONICET). Intendente Güiraldes 2160, Ciudad Universitaria - Pabellón II, C1428EGA Ciudad Autónoma de Buenos Aires, Argentina.leticialuci@gl.fcen.uba.ar; ceciliacataldo@gl.fcen.uba.ar

El Miembro Pilmatué (Formación Agrio) comprende sedimentitas marinas sobre las que, en la región central de la Cuenca Neuquina se dispone una cuña de mar bajo (Miembro Avilé). Hacia el borde austral de la cuenca, el Miembro Avilé no se registra como tal; por lo tanto resulta de interés analizar la transición entre el Miembro Pilmatué y el Miembro Agua de la Mula. En la localidad de Arroyo Covunco se identifica un nivel de arcilitas amarillentas que equivaldría al Miembro Avilé. En las capas infrayacentes al mismo, se colectaron 51 ejemplares del gastrópodo Ampullina (Ampullinidae), realizándose un análisis tafonómico preliminar de los mismos enfocado en la incrustación. El 90\% de los ejemplares sufrió incrustación tanto interna (63\%) como externa (85\%); en promedio cada ejemplar presenta 19 incrustantes. Se observaron sobrecrecimientos entre 6 pares de taxones incrustantes, tanto en la superficie interna como externa de la conchilla. Entre los doce taxones incrustantes, las ostras predominan, seguidas por poliquetos y bryozoos; se hallan asimismo corales coloniales. Los parámetros calculados de incrustación son elevados en comparación a lo observado para otros moluscos colectados hacia el tope del Miembro Pilmatué, sugiriendo un mayor tiempo de exposición de los gastrópodos en esta localidad; ciertos rasgos de las capas muestreadas, como la presencia de nódulos arenosos incrustados sugieren un episodio de omisión. Se proyecta incorporar más muestras al análisis e incorporar otros indicadores, con el fin de caracterizar la historia tafonómica de Ampullina en el techo del Miembro Pilmatué en Arroyo Covunco e inferir el paleoambiente.

\section{ASPECTOS SOBRE LA PRESERVACIÓN DE LOS CONCHOSTRACOS}

\author{
M. Monferran ${ }^{1}$, V. Jiménez ${ }^{1}$ y O. Gallego ${ }^{1}$
}

${ }^{1}$ Centro de Ecología Aplicada del Litoral (CECOAL) - Consejo Nacional de Investigaciones Científicas y Tecnológicas (CONICET) y Universidad Nacional del Nordeste. Ruta 5, km 2,5, 3400 Corrientes, Argentina.monfdm@gmail.com; victoriajimenez70@yahoo.com.ar; osflogallego@gmail.com

Los "conchostracos" o espinicaudados aparecen en el registro fósil desde los 380 millones de años, y son muy frecuentes en el Paleozoico superior y Mesozoico. ¿A qué se debe el éxito de su registro? Generalmente, el caparazón es el único elemento que se conserva, y por lo tanto, es crucial conocer sus propiedades físico-químicas en los actuales para interpretar la preservación de los fósiles. De esta manera, a través de la recopilación bibliográfica e interpretación de los datos, el objetivo de este trabajo es evaluar la influencia que tienen las propiedades del caparazón en la conservación de los espinicaudados. Los estudios de resistencia en caparazones actuales demuestran su gran capacidad de flexibilidad y resistencia a numerosos procesos destructivos (incluidos el transporte, la descomposición, la compactación y la desecación). Por otro lado, los estudios con espectrometría de energía de rayos X y Raman indican que los caparazones actuales están compuestos de fosfato de calcio y carbonato de calcio. En el registro fósil, estos elementos también son reportados en caparazones junto a otros elementos químicos incorporados durante la fosilización, de hecho, la mayoría de los espinicaudados fósiles presentan mineralizaciones en sus caparazones. Estos resultados sugieren que los procesos post mortem previos al enterramiento no tendrían un rol importante en la producción de sesgo tafonómico para el registro fósil. Por lo tanto, las propiedades químicas y mecánicas de los caparazones son factores determinantes en la conservación de los espinicaudados fósiles, y ayudarían a comprender mejor la historia del registro fósil de este grupo. 


\title{
THE TREMADOCIAN RHYNCHONELLIFORM BRACHIOPOD SHELL BEDS OF NW ARGENTINA FROM A PALAEOECOLOGICAL VIEW
}

\author{
D.F. Muñoz', A. Bignon ${ }^{1}$, J.L. Benedetto', And B.G. Waisfeld ${ }^{1 *}$
}

${ }^{1}$ Centro de investigaciones en Ciencias de la Tierra (CICTERRA)-Consejo Nacional de Investigaciones Científicas y Técnicas (CONICET) and Facultad de Ciencias Exactas, Físicas y Naturales, Universidad Nacional de Córdoba. Av. Vélez Sársfield 1611, X5016CGA, Córdoba, Córdoba, Argentina.df.munoz@conicet.gov.ar; arnaudbignon@yahoo.fr; juan.benedetto@unc.edu.ar; bwaisfeld@unc.edu.ar

Occupation of shallow environments by rhynchonelliform brachiopods is rare during the Lower Ordovician. However, in the NW Argentina basin the group, especially Tarfa purmamarcaensis Benedetto, occupied high-energy environments as early as the Tremadocian. This species forms up to $15 \mathrm{~cm}$ thick monospecific concentrations in shoreface and offshore transition settings, and polytypic pavements in relatively deeper water offshore environments. Origin of such concentrations could be linked to the population dynamics of T. purmamarcaensis. The geometric morphometrics analysis allowed differentiating four growth stages in the ontogeny of the species. The juvenile phases are absent in high-energy proximal environments while no representatives of the fourth phase (hypermature adults) have been found in the open platform deposits. On the other hand, measurement of a large number of shells showed that juveniles are scarce in the shoreface while adults are almost absent in the offshore. Although concentrations are usually linked to physical processes (storm events), the low taphonomic indices of the shells suggest that transport was not a significant factor. Morphological differences (i.e., development of cardinal canals, ribs incurved posterolaterally) in specimens from different environments suggest that the population dynamics could have been the main cause in generating different concentrations. According to the source-sink model, high productivity of brachiopods in the shoreface environment and a passive transport of larvae to the offshore might explain not only the thicker shallow-water concentrations but also the differences in shell growth of populations inhabiting these environments.

*Financial support by Agencia Nacional de Promoción Científica y Tecnológica (ANPCyT-FONCyT) PICT 2016-0558. This is a contribution to the IUGS-IGCP project 653 'The onset of the Great Ordovician Biodiversification Event'.

\section{A THOUSAND WAYS TO DIE: EXCEPTIONAL PRESERVATION OF SEA STARS ON A PALEOSUR- FACE FROM LA MESETA FORMATION (EOCENE, ANTARCTIC PENINSULA)}

\author{
E.E. Palópolo1', S. Brezina ${ }^{1}$, S. Casadio¹, S. Santillana², and M. Griffin ${ }^{3 *}$ \\ ${ }^{1}$ Instituto de Investigación en Paleobiología y Geología (IIPG)-Consejo Nacional de Investigaciones Científicas y Tecnológicas (CONICET), \\ Universidad Nacional de Río Negro. Av. Roca 1242, R8332EXZ Gral. Roca, Río Negro, Argentina. eepalopolo@unrn.edu.ar; sbrezina@unrn.edu.ar; \\ scasadio@unrn.edu.ar \\ 2Instituto Antártico Argentino. 25 de Mayo 1143, B1650HMK San Martín, Buenos Aires, Argentina. ssantillana@dna.gov.ar \\ ${ }^{3}$ Consejo Nacional de Investigaciones Científicas y Tecnológicas (CONICET). División Paleozoología Invertebrados, Facultad de Ciencias Natu- \\ rales y Museo, Universidad Nacional de La Plata, Unidades de Investigación Anexo Museo. Avenida 122 y 60, B1904 La Plata, Buenos Aires, \\ Argentina.mgriffin@fcnym.unlp.edu.ar
}

A single paleosurface within the Cucullaea I Allomember (La Meseta Formation, Eocene) exposed in Marambio (Seymour) Island, Antarctica, yielded exceptionally well-preserved starfishes. This allomember was deposited in a sandy to muddysandy tidal flat environment, associated with lenticular densely packed shell-beds with erosive bases interpreted as the infilling of small tidal channels. Fourty-five specimens were identified and assigned to Zoroaster aff. Z. fulgens Blake and Zinsmeister. Individuals were preserved with complete discs, articulated proximal and distal parts of rays, and spines, preservation considered exceptional for fossils in the Class Asteroidea. Five posture categories were recognized among the sea stars: 1) resting position, with straight extended arms; 2) pseudocopulation posture, with superimposed discs and alternated arms; 3) trackeby currents, with curved and irregularly arranged arms; 4) escape posture, with one or two leading arms raised and the others curved downward; and 5) oral side up, with arms extended and slightly curved upward. These postures are similar to those known for living starfishes. The exquisite preservation (i.e., almost all specimens lack signs of disarticulation; with most spines, spinelets, pedicellariae and terminal ossicles in life position) allow to infer that the starfishes were simultaneously killed and buried by a rapid event. This kind of exceptional preservation of starfishes is the third record in the world and the first from Antarctica.

*Financial support by Instituto Antártico Argentino, the Project PI UNRN 40-A-559 and a PhD Fellowship from Consejo Nacional de Investigaciones Científicas y Tecnológicas (CONICET) to Evangelina Palópolo. 


\title{
PLANT TAPHONOMY FROM THE AGUA DE LA ZORRA FORMATION (UPPER TRIASSIC) MENDOZA, ARGENTINA
}

\author{
T.E. Pedernera ${ }^{1}$, A.C. Mancuso ${ }^{1}$, E.G. Ottone ${ }^{2}$, and C.A. Benavente ${ }^{1 *}$
}

${ }^{1}$ Instituto Argentino de Nivología, Glaciología y Ciencias Ambientales (IANIGLA), Centro Científico Tecnológico Mendoza-Consejo Nacional de Investigaciones Científicas y Tecnológicas (CONICET). Avenida Ruiz Leal s/n, 5500 Mendoza, Mendoza, Argentina. tpedernera@mendoza-conicet.gob.ar; amancu@mendoza-conicet.gob.ar; cebenavente@gmail.com

"Instituto de Estudios Andinos "Don Pablo Groeber" (IDEAN), Universidad de Buenos Aires-Consejo Nacional de Investigaciones Científicas y Tecnológicas (CONICET). Intendente Güiraldes 2160, Ciudad Universitaria - Pabellón II, C1428EGA Ciudad Autónoma de Buenos Aires, Argentina. ottone@gl.fcen.uba.ar

The Agua de la Zorra Formation represents a Triassic fluvial-lacustrine environment, with episodic subaqueous incursions of lava flows, recorded at Paramillos de Uspallata locality (north of Mendoza Province). The aim of this contribution is to conduct a taphonomic study of the fossil plants recovered. We carried out a systematic sampling of plant remains. The remains were identified and the taphonomic attributes characterized. A cluster analysis was carried on to identify the taphonomic modes. We identified five taphonomic modes: AZ-A (carbonaceous debris), AZ-B (isolated carbonaceous compressions), AZ-C (isolated impressions), AZ-D (packaged impressions) and AZ-E (articulate and very large fronds). The modes AZ-A and $A Z-B$ were the best represented (58\% and $27 \%$ ) and are interpreted as allochthonous and parautochthonous remains of unidentified specimens, leaves, stems and ovules/seeds. They were transported by wind or flotation and are associated to the offshore facies. The AZ-C and AZD were less represented modes (11\% and 1\%) and are interpreted as allochthonous and parautochthonous remains of leaves and stems. They were transported by water flows and flotation and were associated to prodelta facies. The AZ-E mode (3\%) is interpreted as parautochthonous remains of fronds transported short distances by flotation or low energy flows and this mode was found linked to the offshore and prodelta facies. The taphonomic features of the plant remains in the Agua de la Zorra Formation are similar to the classics models of leaf deposition in lacustrine systems of other Triassic deltaic-lacustrine sequences from Argentina in spite of the incursions of lava flows.

*Financial support by grants PICT-2013-0805 (Agencia Nacional de Promoción Científica y Tecnológica) and PIP 112-201501-00613-CONICET (Consejo Nacional de Investigaciones Científicas y Técnicas).

\section{PRELIMINAR TAPHONOMICAL ANALYSIS OF TRIASSIC BONES FROM LOS COLORADOS FOR- MATION (LA RIOJA PROVINCE - ARGENTINA)}

\author{
M.L. Pérez ${ }^{1,2}$, A. Otero ${ }^{2,3}$, V. Krapovickas ${ }^{4}$, P. Alonso5 ${ }^{5}$, J.M. Leardi ${ }^{4}$, L. Gaetano ${ }^{4}$, and C. Apaldetti ${ }^{6 *}$
}

${ }^{1}$ División Paleozoología Invertebrados, Facultad de Ciencias Naturales y Museo, Universidad Nacional de La Plata, Unidades de Investigación Anexo Museo. Avenida 122 y 60, B1904 La Plata, Buenos Aires, Argentina. pilosaperez@gmail.com

${ }^{2}$ Consejo Nacional de Investigaciones Científicas y Tecnológicas (CONICET).

${ }^{3}$ División Paleontología Vertebrados, Unidades de Investigación Anexo Museo, Facultad de Ciencias Naturales y Museo, Universidad Nacional de La Plata. Av. 122 y 60, B1904 La Plata, Buenos Aires, Argentina. alexandros.otero@gmail.com

"Instituto de Estudios Andinos "Don Pablo Groeber" (IDEAN), Universidad de Buenos Aires-Consejo Nacional de Investigaciones Científicas y Tecnológicas (CONICET). Intendente Güiraldes 2160, Ciudad Universitaria - Pabellón II, C1428EGA Ciudad Autónoma de Buenos Aires, Argentina.veronicakrapovickas@gmail.com; juanmartinleardi@gmail.com; leandrocg1@gmail.com

${ }^{5}$ Área Sedimentología, Instituto de Geociencias Básicas, Aplicadas y Ambientales de Buenos Aires (IGEBA), Consejo Nacionald de Investigaciones Científicas y Tecnológicas (CONICET)-Universidad de Buenos Aires. Intendente Güiraldes 2160, Pabellón II, Piso 1, C1428EGA Ciudad Autónoma de Buenos Aires, Argentina.pablojoaquin3@gmail.com

${ }^{6}$ Centro de Investigaciones de la Geósfera y la Biósfera (CIGEOBIO)-Consejo Nacional de Investigaciones Científicas y Tecnológicas (CONICET)Instituto y Museo de Ciencias Naturales, Universidad Nacional de San Juan. Av. España 400 (Norte), J5400DNQ San Juan, San Juan, Argentina. capaldetti@unsj.edu.ar

Los Colorados Formation is a worldwide recognized continental unit deposited in the Ischigualasto-Villa Unión basin in the San Juan and La Rioja provinces, northwestern Argentina. It is mainly composed of lenticular sandstone beds and laterally extensive laminated to massive mudstone units, recording sedimentation in different fluvial settings. These rocks represent a case study for the abundance and diversification events that affected Upper Triassic tetrapods. Although the tetrapod fauna recovered from this unit is relatively well-known, their taphonomic modes are scarcely understood. In this sense, we present a preliminary petrographic and X-ray diffraction analyses of both epiphyses and diaphyses of bone remains collected in Los Colorados Formation, which allowed us to interpret the damaging processes that acted on the bone tissue. 
The most destructive taphonomical process that affected the bones was the chemical interaction with circulating fluids during fossil-diagenesis. Thin section of the epiphyses shows preserved trabecular bone with cracks perpendicular to the bone fibers, obtured cavities filled with calcite set in two cycles: microgranular on the periphery and polycristaline euhedral on the centre. Sub-periosteal bone, however, is relatively well-preserved. Diaphyses present a highly altered trabecular bone and obtured cavities with calcite in three cycles: two microgranular peripherical rows and large euhedral crystals in the internal lumen. Sub-periosteal bone is mostly altered. X-ray diffraction shows predominance of calcite in the epiphysis, whereas gypsum is mostly present in the diaphysis. Summing up, diagenetic cracks allowed the circulation solutions (rich in calcite and gypsum) in the bone, expanding and destroying it.

\title{
ANÁLISIS TAFONÓMICO CUANTITATIVO Y GÉNESIS DE UNA CONCENTRACIÓN FÓSIL DEL MIOCENO TEMPRANO DE LA CUENCA AUSTRAL
}

\author{
G. Pineda-Salgado ${ }^{1}$, A. Parras ${ }^{1}$ y M. Griffin $2,3 *$ \\ 1'Instituto de Ciencias de La Tierra y Ambientales de La Pampa (INCIT AP), Consejo Nacional de Investigaciones Científicas y Tecnológicas-Univer- \\ sidad Nacional de La Pampa. Uruguay 151, L6300CLB Santa Rosa, La Pampa, Argentina.pineda.salgado.g@gmail.com; anaparras@conicet.gov.ar \\ ${ }^{2}$ Consejo Nacional de Investigaciones Científicas y Tecnológicas (CONICET). División Paleozoología Invertebrados, Facultad de Ciencias Natu- \\ rales y Museo, Universidad Nacional de La Plata, Unidades de Investigación Anexo Museo. Avenida 122 y 60, B1904 La Plata, Buenos Aires, \\ Argentina.mgriffin@fcnym.unlp.edu.ar
}

Las concentraciones fósiles pueden constituir depósitos complejos debido a los diversos procesos biológicos y sedimentológicos que las originan; sin embargo, el estudio de su biofábrica y el análisis cuantitativo de los índices tafonómicos de sus elementos individuales brindan información acerca de su génesis. Con este objetivo, se analizó una concentración de la Formación Monte León, en la localidad Cabeza de León, compuesta principalmente por moluscos. Se describió la concentración en campo y se procesaron dos muestras en laboratorio, las cuales fueron tamizadas con tres mallas de diferente apertura $(5,66 \mathrm{~mm}, 1,19 \mathrm{~mm}$ y 0,71 mm). Los especímenes retenidos $(n=1.775)$ fueron determinados a nivel de género, evaluando en cada uno el índice de fragmentación, corrasión, bioerosión y redondeamiento; para los bivalvos se consideró la preservación del margen ventral. La concentración es politípica, predominando especímenes de gasterópodos; de algunas especies se preserva un amplio espectro de edad. La preservación en las mallas 0,71 y 1,19 fue similar, con fragmentación y corrasión moderadas, alto redondeamiento y baja bioerosión; el margen ventral fue principalmente discontinuo. En la malla 5,66 la fragmentación y corrasión fueron altas con bioerosión moderada a alta. Los índices tafonómicos también fueron analizados en relación con la fragmentación; los fragmentos mostraron índices más altos en todas las mallas. Las características tafonómicas y paleoecológicas sugieren que la concentración estudiada es de origen biológico-sedimentológico, asociada a un ambiente marino somero y conformada principalmente por elementos parautóctonos. Los altos índices tafonómicos de los fragmentos sugieren mayor antigüedad y múltiples episodios de retrabajo.

*Proyecto subsidiado por ANPCyT (PICT 2016-1872) y FCEyN-UNLPam (PI08G). 


\title{
ESTUDIO TAFONÓMICO DE ASOCIACIONES DE VERTEBRADOS DEL MIOCENO TARDÍO DEL VALLE ARGENTINO, PROVINCIA DE LA PAMPA, ARGENTINA
}

\author{
R. Sostilloo $0^{1,2}$ y C.I. Montalvo ${ }^{1 *}$
}

${ }^{1}$ Facultad de Ciencias Exactas y Naturales, Universidad Nacional de La Pampa. Uruguay 151, L6300CLB Santa Rosa, La Pampa, Argentina. renata.sostillo@gmail.com; cmontalvo@exactas.unlpam.edu.ar

${ }^{2}$ Consejo Nacional de Investigaciones Científicas y Tecnológicas (CONICET).

Los niveles de la Formación Cerro Azul aflorantes en Salinas Grandes de Hidalgo y Laguna Chillhué, en el Valle Argentino, centro-este de La Pampa (Argentina), corresponden a depósitos fluviales efímeros con paleosuelos intercalados. Las asociaciones faunísticas recuperadas de estos yacimientos corresponden al Mioceno Tardío y fueron objeto de evaluaciones tafonómicas con resultados similares en ambas localidades. Esta similitud indica que, aun aceptando la interpretación previa de una mínima diferencia temporal entre ellas, los procesos actuantes son semejantes. Se identificaron atributos tafonómicos que se pueden vincular con procesos ocurridos en medios ácueos, entre ellos se destacan la alta frecuencia de algunos tipos de elementos esqueléticos (cráneos, mandíbulas) y los diferentes grados de articulación representados (predominio de los elementos aislados). Por otro lado, la ocurrencia de impregnaciones, corrosiones, desarrollo de concreciones, rizolitos, encostramientos sobre los restos y trazas de raíces, se pueden vincular con procesos pedogenéticos. Los roedores caviomorfos, notoungulados y xenartros, indican una dieta basada principalmente en plantas C3, dato que avala la presencia de un paleoambiente abierto con clima templado. En el caso de Salinas Grandes de Hidalgo, esta interpretación también se apoya en el registro de icnotaxones indicativos de áreas abiertas. Pese a la gran similitud ambiental inferida entre ambas asociaciones, en Laguna Chillhué la alta proporción de restos impregnados por óxidos de manganeso corrobora una mayor participación de agua en los procesos tafonómicos actuantes, sugerencia avalada también por la presencia de restos de carpinchos, roedores asociados a cuerpos de agua.

*Proyecto financiado por 06-G, Facultad de Ciencias Exactas y Naturales, UNLPam.

\section{TAFONOMÍA DE VERTEBRADOS PRESERVADOS EN DEPÓSITOS DE CENIZA VOLCÁNICA DEL MIOCENO TEMPRANO (FORMACIÓN SANTA CRUZ) DE PATAGONIA, ARGENTINA}

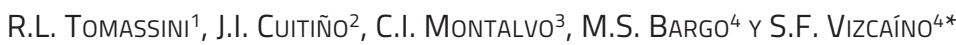

'Departamento de Geología, Universidad Nacional del Sur (UNS), Instituto Geológico del Sur (INGEOSUR)-Consejo Nacional de Investigaciones Científicas y Tecnológicas (CONICET). Av. Alem 1253 cuerpo B' $1^{\circ}$ Piso, B8000CPB Bahía Blanca, Buenos Aires, Argentina. rodrigo.tomassini@yahoo.com.ar ${ }^{2}$ Instituto Patagónico de Geología y Paleontología (IPGP), Centro Nacional Patagónico (CENPAT)-Consejo Nacional de Investigaciones Científicas y Tecnológicas (CONICET). Boulevard Brown 2915, U9120ACD Puerto Madryn, Chubut, Argentina. jcuitino@cenpat-conicet.gob.ar ${ }^{3}$ Facultad de Ciencias Exactas y Naturales, Universidad Nacional de La Pampa. Uruguay 151, L6300CLB Santa Rosa, La Pampa, Argentina. cmontalvo@exactas.unlpam.edu.ar

${ }^{4}$ División Paleontología de Vertebrados, Unidades de Investigación Anexo Museo, Facultad de Ciencias Naturales y Museo, Universidad Nacional de La Plata. Av. 60 y 122, B1904 La Plata, Buenos Aires, Argentina.msbargo@fcnym.unlp.edu.ar; vizcaino@fcnym.unlp.edu.ar

Las evaluaciones tafonómicas de vertebrados continentales se han incrementado, pero aún existen contextos preservacionales poco explorados. En este marco, se realizó el análisis tafonómico de una asociación recuperada en un nivel de toba fina secundaria (Toba CO; 17,41 Ma, Burdigaliense) de la Formación Santa Cruz, aflorante en la localidad Cañadón de Las Vacas. Se recuperaron restos de Paucituberculata (Palaeothentidae, Microbiotheriidae), Xenarthra (Peltephilidae, Dasypodidae, Propalaehoplophoridae, Megatherioidea), Rodentia (Neoepiblemidae, Octodontoidea, "Eocardiidae", Cavioidea incertae sedis), Notoungulata (Toxodontidae, Interatheriidae, Hegetotheriidae) y Litopterna (Proterotheriidae), escasos Anura y coprolitos. El Número Mínimo de Individuos fue 70 y el Número Mínimo de Elementos 281. Dientes aislados (31\%), hemimandíbulas $(27 \%)$ y hemimaxilares $(17 \%)$ fueron los elementos esqueléticos más frecuentes, los restantes estaban ausentes o presentaban valores muy bajos. Predominaron elementos esqueléticos desarticulados y aislados, pero hubo otros articulados y/o asociados. El 75\% de los especímenes estaba incompleto, el 10\% presentaba signos leves de meteorización y no se observaron especímenes con evidencias de abrasión. Las evidencias sugieren poco tiempo de exposición subáerea y escaso o nulo transporte de los restos. La acumulación de ceniza habría favorecido el rápido enterramiento y preservación, en el lugar de muerte o cercano a este, tanto de restos previamente acumulados en un ambiente de llanura de inundación como de aquellos contemporáneos a la depositación secundaria del material volcánico. Se registraron restos con marcas de raíces e impregnación por óxidos de manganeso, atributos vinculados a procesos pedogenéticos. El alto grado de rotura de la asociación se atribuye a procesos de reexposición recientes.

*Proyecto financiado por PICT 2013-0389; UNLP 11/N750; NGS CP-030R-17. 


\title{
ESTUDIO TAFONÓMICO DE LAS OCURRENCIAS EN MASA DE OSTRAS AETOSTREON SP. (OMOS) EN LA TRANSICIÓN ENTRE LAS FORMACIONES VACA MUERTA Y MULICHINCO, VALANGINIANO TEMPRANO, PROVINCIA DE NEUQUÉN
}

\author{
A.G. TOSCANO ${ }^{1}$, D. LAZO ${ }^{1}$ y E. SCHWARZ ${ }^{2 *}$ \\ ${ }^{1}$ Instituto de Estudios Andinos "Don Pablo Groeber" (IDEAN), Universidad de Buenos Aires-Consejo Nacional de Investigaciones Científi- \\ cas y Tecnológicas (CONICET). Intendente Güiraldes 2160, Ciudad Universitaria - Pabellón II, C1428EGA Ciudad Autónoma de Buenos Aires, \\ Argentina.atoscano@gl.fcen.uba.ar; dlazo@gl.fcen.uba.ar \\ ${ }^{2}$ Centro de Investigaciones Geológicas (CIG), Universidad Nacional de La Plata-Consejo Nacional de Investigaciones Científicas y Tecnológicas \\ (CONICET). Diagonal 113 n²74, B1904DPK1900 La Plata, Buenos Aires, Argentina. eschwarz@cig.museo.unlp.edu.ar
}

Los OMOs son elementos frecuentes dentro del Grupo Mendoza de la Cuenca Neuquina. En este caso, se documentaron numerosos OMOs inmersos en facies finas, conformados por ostras del género Aetostreon Bayle en la transición entre las Formaciones Vaca Muerta y Mulichinco, dentro de la Zona de Lissonia riveroi (Valanginiano temprano, localidad La Herradura, Neuquén). Estos son prácticamente monoespecíficos, con gran abundancia de ostras y raras apariciones de otros taxones (gastrópodos, trigonias y serpúlidos). A partir del análisis tafonómico y estratigráfico realizado, los OMOs fueron interpretados como biogénicos. Dentro de cada OMO se diferenciaron 5 biohermas apilados (entre 10 y 20 m de ancho y 3 m de espesor máximo), separados por bancos delgados pelíticos que indicarían períodos de inhibición en el crecimiento vertical de cada bioherma. Estos presentan geometría lenticular, disminuyendo lateralmente en espesor y abundancia de ostras, gradando a sedimentos finos donde la fauna cambia, con la aparición de bivalvos del género Aphrodina y gastrópodos matíldidos. Lateralmente se diferenciaron al menos 4 OMOs de este tipo, distribuidos a lo largo de 300 m de afloramiento. Estos niveles portadores de OMOs fueron registrados también en la localidad Cerro La Parva (aproximadamente a 60 km al SO de La Herradura), indicando una amplia distribución de los mismos en los fondos marinos de la Cuenca Neuquina. La aparición de estos OMOs en ese momento particular de la cuenca indicaría un conjunto de parámetros ambientales específicos (salinidad e ingreso de nutrientes), asociados al inicio de la regresión marina marcada por la Formación Mulichinco.

*Proyecto financiado por IDEAn C-150, Lazo Préstamo BID PICT 2015-1381 


\title{
SIMPOSIO \\ "PALEOBIOGOEGRAFÍA DE GONDWANA"
}

\section{THE FIRST PHYTOSAUR FROM SUB-SAHARAN AFRICA: CHRONOLOGICAL AND PALAEOECO- LOGICAL IMPLICATIONS}

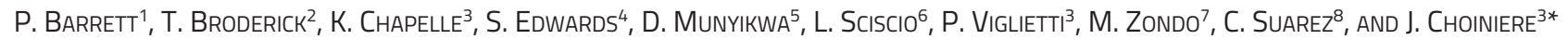 \\ 1Natural History Museum. Cromwell Road, SW7 5BD, London, London, UK. p.barrett@nhm.ac.uk \\ ${ }^{2}$ NA.19 Jenkinson Road, NA, Harare, NA, Zimbabwe. makari@zol.co.zw \\ 3Evolutionary Studies Institute, University of the Witwatersrand. 2050 Johannesburg, Gauteng, South Africa. kimi.chapelle@gmail.com; \\ pia.viglietti@gmail.com; jonah.choininere@wits.ac.za \\ ${ }^{4}$ Musango Safari Camp. Musango Island, NA, Lake Kariba, NA, Zimbabwe. steve.edwards.musango@gmail.com \\ ${ }^{5}$ National Museums and Monuments.107 Rotten Row, NA, Harare, NA, Zimbabwe. dtonmunyikwa@gmail.com \\ 6University of Johannesburg, Auckland Park Kingsway Campus. NA, Johannesburg, Gauteng, South Africa. I.sciscio@gmail.com \\ 7The Natural History Museum of Zimbabwe. Park Road, NA, Bulawayo, NA, Zimbabwe. miczondo@yahoo.com \\ 8University of Arkansas. 340 N. Campus Drive, 72701, Fayetteville, Arkansas, United States of America. casuarez@uark.edu
}

Phytosaur material is uncommon in Gondwana and has previously been reported only from Brazil, India, Madagascar and Morocco. However, recent fieldwork in the Tashinga Formation (upper Karoo Supergroup) of the Lake Kariba region, Zimbabwe, has recovered the first phytosaur material from sub-Saharan mainland Africa, which consists of partial snouts, teeth and osteoderms. This material extends the distribution of the clade significantly, showing that the group occurred at much higher southerly palaeolatitudes than previously realised. Radioisotopic dates obtained from LA-ICPMS analyses of detrial zircons suggest that the Tashinga Formation was of Late Triassic (Norian) age, consistent with the presence of phytosaurs, which are widely regarded as Norian-Rhaetian index fossils. The phytosaurs co-occur with numerous lungfish, metoposaurid temnospondyls and a terrestrial archosaur. Fossil wood is also exceptionally abundant at these localities. In addition to faunal evidence, these localities record sedimentological indicators of deposition in a mesic, well-watered palaeoenvironment and they represent the first record of aquatic freshwater ecosystems from the Karoo Supergroup of southern and eastern Africa. In many ways, the Tashinga Formation fauna can be viewed as a Gondwanan analogue of similar Late Triassic faunas in Laurasia, such as those recovered from the Chinle Formation of the southwestern USA.

${ }^{*}$ Financial support by Palaeontological Scientific Trust (PAST) and its Scatterlings of Africa programmes (to JNC); National Research Foundation (NRF) African Origins Platform Grant (98800) (to JNC); Natural. History Museum Earth Sciences Departmental Investment Fund (to PMB); the DST /NRF Centre of Excellence (CoE2017-046) in Palaeoscience (to JNC). 


\title{
NEW FOSSIL REMAINS FROM THE LAGO COLHUÉ HUAPI FORMATION (CONIACIAN-MAAS- TRICHTIAN): INCREASING THE RECORD OF VERTEBRATE DIVERSITY AND THE PALEOECO- LOGICAL KNOWLEDGE FOR THE LATE CRETACEOUS OF PATAGONIA
}

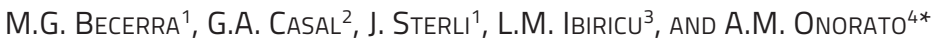 \\ ${ }^{1}$ Consejo Nacional de Investigaciones Científicas y Tecnológicas (CONICET)-Museo Paleontológico Egidio Feruglio. Fontana 140, U9100GYO \\ Trelew, Chubut, Argentina.mbecerra@mef.org.ar; jsterli@mef.org.ar \\ 2Departamento de Geología, Facultad de Ciencias Naturales y Ciencias de la Salud, Universidad Nacional de la Patagonia San Juan Bosco. Ruta \\ Provincial Nro 1 - Ciudad Universitaria - Km 4, 9005 Comodoro Rivadavia, Chubut, Argentina.paleogac@yahoo.com.ar \\ ${ }^{3}$ Instituto Patagónico de Geología y Paleontología (IPGP), Centro Nacional Patagónico (CENPAT)-Consejo Nacional de Investigaciones Cientí- \\ ficas y Tecnológicas (CONICET). Boulevard Brown 2915, U9120ACD Puerto Madryn, Chubut, Argentina. ibiricu@cenpat-conicet.gob.ar \\ ${ }^{4}$ Facultad de Ciencias Naturales, Universidad Nacional de la Patagonia San Juan Bosco. 9 de Julio y Belgrano, 9100 Trelew, Chubut, Argentina. \\ onoratoandrea@hotmail.com
}

In this communication we present a fossil association found in a new locality of the Lago Colhué Huapi Formation of Chubut, Central Patagonia. This site is located $16.65 \mathrm{~km}$ south-eastern of the origin of the Chico River and $3.65 \mathrm{~km}$ south of the stratotype locality of the Formation. The fossils were recovered about $1.5 \mathrm{~m}$ below the erosive contact with the base of the Salamanca Formation. This association mainly includes postcranial remains of Hadrosauridae and Testudinata found in successive layers of green-greyish to reddish mudstones with carbonized logs, interpreted as distal floodplains. The hadrosaurid remains comprise vertebrae representing all regions, fragments from the pectoral and pelvic girdles and fragmentary fore and hind limb bones. Several limb bone fragments, although lacking of overlapping morphology, show a marked difference in size, supporting the coexistence of different ontogenetic stages of a Hadrosauridae representative. The turtle remains are fragmentary but can be identified belonging to two different groups, supporting the coexistence of different sized Testudinata in this association. A large, undecorated peripheral plate is identified as Testudinata indet. In addition, a small, decorated peripheral plate is identified as Chelidae indet., however its size and ornamentation resembles Hydromedusa $\mathrm{sp}$. and Yaminuechelys sp. This fossil association increases the knowledge of the vertebrate diversity and paleoecology of the formation, resulting in the first record of different ontogenetic stages of Hadrosauridae, and the first evidence of different fresh-water turtles with contrasting sizes interacting at the same environment.

*Fieldwork was made thanks to a grant awarded from the Jurassic Foundation (to MGB).

\section{NEW ICHNOLOGICAL MATERIAL FROM THE VERA FORMATION (LOS MENUCOS COMPLEX, TRIASSIC), PUESTO VERA LOCALITY, RÍO NEGRO PROVINCE, ARGENTINA: A FIRST ACCOUNT}

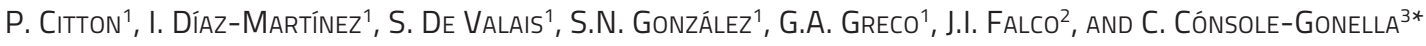

${ }^{1}$ Instituto de Investigación en Paleobiología y Geología (IIPG) - Consejo Nacional de Investigaciones Científicas y Tecnológicas (CONICET), Universidad Nacional de Río Negro. Av. Roca 1242, R8332EXZ Gral. Roca, Río Negro, Argentina. pcitton@unrn.edu.ar; inaportu@hotmail.com; sdevalais@yahoo.com.ar; santiagonicolas.gonzalez@gmail.com; ggreco@unrn.edu.ar

${ }^{2}$ Instituto Geológico del Sur (INGEOSUR)-Consejo Nacional de Investigaciones Científicas y Tecnológicas (CONICET), Universidad Naiconal del Sur. San Juan 670, B8000ICN Bahia Blanca, Buenos Aires, Argentina. juan.falco@uns.edu.ar

${ }^{3}$ Instituto Superior de Correlación Geológica (INSUGEO), Universidad Nacional de Tucumán-Consejo Nacional de Investigaciones Científicas y Tecnológicas (CONICET). Miguel Lillo 205, T4000JFE San Miguel de Tucumán, Tucumán, Argentina. carlosconsole@csnat.unt.edu.ar

Los Menucos locality, in the north-western sector of the North Patagonian Massif (Río Negro province, Argentina), is prominent for having returned one of the most significant Triassic tetrapod ichnofauna of southern South America. A volcaniclastic succession mainly composed of dacitic to rhyolitic ignimbrites, mesosilicic lavas and subordinate sedimentary rocks, is exposed there. The tetrapod footprints are preserved in volcaniclastic and sedimentary strata of the Vera Formation, which constitutes, together with the overlying Sierra Colorada Formation, the Los Menucos Complex. The ichnofauna is mainly documented by small and large pentadactyl tracks respectively referable to as Dicynodontipus and Pentasauropus. The Dicynodontipus tracks originally came from the Tscherig quarry (west of Los Menucos town), while the Pentasauropus specimens came from the Yancaqueo quarry (east of Los Menucos town). In both cases, the exact stratigraphic position of the material, up to date, is unknown. Here, we present a new ichnosite from the thin sedimentary sequence of Puesto Vera Formation in its type locality. The material consists of 14 footprints of about $5 \mathrm{~cm}$ in overall length. The best-preserved tracks are pentadactyl with short and straight digits and show a central, well-developed heel-trace, resembling footprints commonly referred to as Dicynodontipus. The new finding is very important seeing that it allows, for the first time, positioning tetrapod tracks within the Vera Formation.

*Financial support by PIP 576. 


\title{
FLORAS HOMÓLOGAS EN EL JURÁSICO TEMPRANO DE ARGENTINA Y ANTÁRTIDA: PALEO- BIOGEOGRAFÍA EN EL SUDOESTE GONDWÁNICO
}

\author{
A. Elgorriaga ${ }^{1}$, I.H. Escapa ${ }^{1}$, R. SCASSO ${ }^{2}$, C.R. Amenabar ${ }^{3,4}$ Y J.M. LIRIO ${ }^{4}$
}

${ }^{1}$ Consejo Nacional de Investigaciones Científicas y Tecnológicas (CONICET). Museo Paleontológico Egidio Feruglio. Avenida Fontana 140, U9100GYO Trelew, Chubut, Argentina.aelgorriaga@mef.org.ar; iescapa@mef.org.ar

'Instituto de Geociencias Básicas, Aplicadas y Ambientales de Buenos Aires (IGeBA), Consejo Nacional de Investigaciones Científicas y Tecnológicas (CONICET)-Departamento de Ciencias Geológicas, Facultad de Ciencias Excatas y Naturales. Universidad de Buenos Aires. Intendente Güiraldes 2160, C1428EGA Ciudad Autónoma de Buenos Aires, Argentina. rscasso@gl.fcen.uba.ar

3Departamento de Ciencias Geológicas, Facultad de Ciencias Exactas y Naturales, Instituto de Estudios Andinos "Don Pablo Groeber" (IDEAN), Universidad de Buenos Aires-Consejo Nacional de Investigaciones Científicas y Tecnológicas (CONICET). Intendente Güiraldes 2160, Ciudad Universitaria - Pabellón II, C1428EGA Ciudad Autónoma de Buenos Aires, Argentina. amenabar@gl.fcen.uba.ar

${ }^{4}$ Instituto Antártico Argentino. Avenida 25 de Mayo 1143, B1650HMK San Martín, Buenos Aires, Argentina. liriojm@gmail.com

El Monte Flora (Bahía Esperanza, Península Antártica) posee una de las asociaciones vegetales clásicas en la literatura paleobotánica y, debido a su enorme valor científico, fue denominado como Zona Antártica Especialmente Protegida. Su tafoflora es una de las más diversas a nivel mundial y su edad ha sido discutida en numerosos estudios, en un rango que abarca desde el Jurásico Temprano al Cretácico. Como consecuencia, las edades de numerosas tafofloras del Hemisferio Sur comparables con la de Monte Flora, han variado de igual manera. Recientemente se realizó un esquema crono-estratigráfico basado en edades absolutas para la Cuenca Cañadón Asfalto, Chubut, datando la localidad de Taquetrén (Formación Lonco Trapial), portadora de una tafoflora ahora con seguridad asignada al Jurásico Temprano (probablemente Pliensbachiano), edad previamente sugerida por co-ocurrencias de taxones vegetales (e.g., Sagenopteris, Goeppertella, Clathropteris, Archangelskya). Estudios de detalle sobre colecciones intensivas realizadas durante la última década en Taquetrén, permitieron corroborar un notable grado de similitud taxonómica entre dicha localidad y aquella de la Península Antártica. Asimismo, durante la temporada de verano de 2018 se realizó una campaña al Monte Flora en la cual se coleccionaron más de mil especímenes, permitiendo reconocer taxones y órganos vegetales claves para realizar comparaciones regionales más precisas (e.g., Williamsonia, Austrohamia). El estudio detallado de estas floras, el cual se expande también a otras floras liásicas de Argentina (e.g., Piedra Pintada, Roca Blanca) representa un primer paso en el objetivo de establecer un esquema biogeográfico y bioestratigráfico regional, en el contexto del Jurásico en Gondwana.

\section{LAS RELACIONES PALEOBIOGEOGRÁFICAS DE LOS TETRÁPODOS CONTINENTALES TRIÁSICOS: UNA REVISIÓN DE LOS AVANCES EN LA TEMÁTICA EN LOS ÚLTIMOS 10 AÑOS}

\section{EZCURRA ${ }^{1}$}

'Museo Argentino de Ciencias Naturales "Bernardino Rivadavia" (MACN)-Consejo Nacional de Investigaciones Científicas y Tecnológicas (CONICET). Av. Ángel Gallardo 470, C1405DJR Ciudad Autónoma de Buenos Aires, Argentina. martindezcurra@yahoo.com.ar

El estudio de la paleobiogeografía triásica es crucial para explorar el efecto que modificaciones en la biodiversidad tuvieron sobre la distribución espacio-temporal de organismos en el pasado. Durante el Triásico, la gran mayoría de los continentes actuales se encontraban formando el supercontinente de Pangea y existían pocas barreras físicas que dificultaran la dispersión de los tetrápodos terrestres. Este contexto paleogeográfico y el registro fósil disponible durante el siglo XX condujeron a varios autores a proponer una fauna global uniforme y cosmopolita durante el Triásico. En las últimas décadas, a partir de un aumento del registro fósil, se ha empezado a observar una variación paleolatitudinal en la distribución de los tetrápodos triásicos, lo cual es congruente con distinciones paleoclimáticas dentro de Pangea y entre las macrofloras del norte y sur del supercontinente. Análisis de reconciliación de árboles recuperaron una distribución cosmopolita para los tetrápodos del Triásico Medio y la aparición de una estructuración paleolatitudinal con eventos cladogenéticos simpátricos a nivel regional durante el Triásico Tardío. Más recientemente, un análisis de redes filogenético cuantificó índices de conectividad biogeográfica para los tetrápodos del Pérmico Medio al Jurásico Temprano. Mediante este análisis se encontraron picos significativos en relación a la presencia de grupos cosmopolitas inmediatamente después de los eventos de extinción masiva del Permo/Triásico y Triásico/Jurásico, y un subsecuente aumento del endemismo durante ambas recuperaciones bióticas. Estos picos fueron el resultado principalmente de la aparición de nuevos taxones ampliamente distribuidos inmediatamente después de ambos eventos extintivos y no de la supervivencia de especies cosmopolitas. 


\title{
DINOFLAGELADOS DEL EOCENO MEDIO EN EL ATLÁNTICO SUDOCCIDENTAL: UN MODELO DE PALEOCIRCULACIÓN PREVIO A LA INSTALACIÓN DE LA CORRIENTE CIRCUMPOLAR AN- TÁRTICA
}

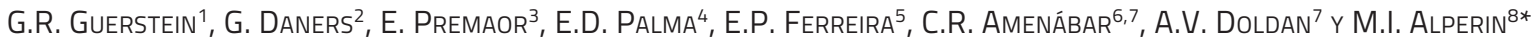

1Departamento de Geologia, Universidad Nacional del Sur, Instituto Geológico del Sur (INGEOSUR)-Consejo Nacional de Investigaciones Científicas y Tecnológicas (CONICET). Av. Alem 1253 cuerpo B' 2 Piso, B8000CPB Bahía Blanca, Buenos Aires, Argentina. raquel.guerstein@uns.edu.ar ${ }^{2}$ Facultad de Ciencias, Universidad de la República (UDELAR). Iguá 4225, 11400 Montevideo, Montevideo, Uruguay. glo@fcien.edu.uy ${ }^{3}$ Laboratório de Palinologia Marleni Marques Toigo, Instituto de Geociências, Universidade Federal do Rio Grande do Sul. Av. Bento Gonçalves 9500, 91540-000 Porto Alegre, Rio Grande do Sul, Brasil.eduardo-premaor@gmail.com

${ }^{4}$ Instituto Argentino de Oceanografía (IADO), Consejo Nacional de Investigaciones Científicas y Tecnológicas (CONICET)-Departamento de Física, Universidad Nacional del Sur. Av. Alem 1253, B8000CPB Bahía Blanca, Buenos Aires, Argentina.uspalma@uns.edu.ar

${ }^{5}$ Centro de Pesquisas e Desenvolvimento Leopoldo A. M. Mello, Petrobras., Av. Horácio Macedo 950, 21941-915 Río de Janeiro, Río de Janeiro, Brasil.elizabete@petrobras.com.br

${ }^{6}$ Instituto Antártico Argentino. 25 de Mayo 1143, B1650HMK San Martín, Buenos Aires, Argentina. amenabar@gl.fcen.uba.ar

"Departamento de Ciencias Geológicas, Facultad de Ciencias Exactas y Naturales, Instituto de Estudios Andinos "Don Pablo Groeber" (IDEAN), Universidad de Buenos Aires-Consejo Nacional de Investigaciones Científicas y Tecnológicas (CONICET). Intendente Güiraldes 2160, Ciudad Universitaria - Pabellón II, C1428EGA Ciudad Autónoma de Buenos Aires, Argentina. anidoldan@gmail.com

${ }^{8}$ Facultad de Ciencias Naturales y Museo, Universidad Nacional de La Plata. Calle 64 s/n (e/ Bv. 120 y Diag. 113), B1904DZA La Plata, Buenos Aires, Argentina.marta.alperin@gmail.com

Los quistes de dinoflagelados (dinoquistes) del Eoceno Medio de la Cenca Austral y Península Antártica están representadas por una asociación endémica dominante en altas latitudes del Hemisferio Sur. Algunos miembros de esta asociación se reconocieron en las cuencas Colorado $\left(\sim 38^{\circ} \mathrm{S}\right)$, Punta del Este $\left(\sim 36^{\circ} \mathrm{S}\right)$ y Pelotas $\left(\sim 30^{\circ} \mathrm{S}\right)$. En las cuencas de Jequitinhonha $\left(\sim 17^{\circ} \mathrm{S}\right)$ y Sergipe $\left(\sim 11^{\circ} \mathrm{S}\right)$, solo se registró la presencia del único miembro de dicha asociación tolerante a aguas templadas. Estudios previos desarrollados en el área de Tasmania relacionaron la presencia de especies endémicas en latitudes medias con un giro subpolar ciclónico favorecido por la obstrucción parcial de los pasajes de Tasmania y Drake. La distribución de dinoquistes en cuencas de la Plataforma del Atlántico Sudoccidental puede ser explicada por un mecanismo similar inducido por un giro subpolar ciclónico en el Atlántico Sur cuya Corriente de Borde Oeste (CBO), iniciada en el talud de la plataforma oeste antártica, seguiría una trayectoria similar a la actual Corriente de Malvinas sobre el talud patagónico. Estudios teóricos recientes indican que una CBO de esta naturaleza inyecta aguas oceánicas hacia la plataforma adyacente e incrementa el flujo de plataforma en la misma dirección. Aguas circum-antárticas y parte de la asociación endémica habrían sido conducidas de este modo hacia el norte. En la actualidad, las aguas subantárticas llegan a su posición de máxima penetración en cercanías de $33^{\circ} \mathrm{S}$, una región conocida como Frente Subtropical de Plataforma. Los registros mencionados permiten sugerir que dicho frente se ubicaría en latitudes más bajas durante el Eoceno Medio.

*Proyecto financiado por PGI 24/F066.

\section{CONÍFERAS Y ANGIOSPERMAS ANATÓMICAMENTE PRESERVADAS EN CONCRECIONES CALCÁREAS DEL CRETÁCICO SUPERIOR, CUENCA DE JAMES ROSS, PENÍNSULA ANTÁRTICA}

\author{
M.L. PIPO ${ }^{1}$, A. IGLESIAS ${ }^{1}$ Y J. BODNAR $2,3 *$ \\ ${ }^{1}$ Instituto de Investigaciones en Biodiversidad y Medioambiente (INIBIOMA), Consejo Nacional de Investigaciones Científicas y Tecnológicas- \\ Universidad Nacional del Comahue (CONICET-UNCO). Quintral 1250, R8400FRF San Carlos de Bariloche, Río Negro, Argentina. \\ laurapipo2@gmail.com; ari_iglesias@yahoo.com.ar \\ ${ }^{2}$ Consejo Nacional de Investigaciones Científicas y Tecnológicas. División Paleobotánica, Museo de La Plata, Facultad de Ciencias Naturales y \\ Museo, Universidad Nacional de La Plata. Paseo del Bosque s/n, B1900FWA La Plata, Buenos Aires, Argentina. jbodnar@fcnym.unlp.edu.ar
}

La Formación Santa Marta (Sección basal del Grupo Marambio), está representada por depósitos de estuario y plataforma de edad Santoniano-Campaniano. Si bien es conocida por fósiles de vertebrados e invertebrados marinos, también preserva organismos continentales originalmente transportados por las corrientes desde el margen Este de la Península Antártica. La flora no abunda en el registro, pero corresponde a una de las pocas evidencias de lo que ocurría durante el Cretácico Superior en este sector del continente, hoy mayormente cubierto por hielo. La flora es diversa y conocida por compresiones de hojas y semillas, permineralizaciones de tallos y leños y palinomorfos. Nuevos hallazgos de concreciones calcáreas marinas del Miembro Beta (Campaniano) se destacan por preservar órganos permineralizados, tanto vegetativos como reproductivos, en conexión orgánica y manteniendo la forma tridimensional. Se estudiaron realizando cortes seriados con la técnica de peel en acetato de celulosa, con la que se pudo recuperar información anatómica de tejidos muy delicados y re- 
construir imágenes tridimensionales. Se diferenciaron cuatro nuevos tipos de follaje de coníferas en base a la forma y disposición de trazas vasculares y canales resiníferos, una angiosperma leñosa carente de vasos y con variante cambial similar al género Sarcandra (Chloranthaceae); y diferentes estructuras internas en semillas y pericarpio de frutos. La completa descripción y comparación de estos taxones, permitirá un mejor entendimiento de las floras de 80 millones de años que vivieron en tan altas latitudes (sometidas al estrés de inviernos oscuros) en el sector continental de la Península Antártica.

*Proyecto financiado por ANPCyT PICT 0-2010-0093. 


\title{
COMUNICACIONES LIBRES
}

\section{ANATOMÍA CRANEAL Y RECONSTRUCCIÓN PALEONEUROLÓGICA DE ANTHROPORNIS GRANDIS WIMAN (AVES, SPHENISCIFORMES), DEL EOCENO DE ANTÁRTIDA}

\author{
C. Acosta Hospitaleche ${ }^{1}$, A.P. Carabajal ${ }^{2}$, A. Ruella ${ }^{1}$ y N. Bauzáa ${ }^{1 *}$ \\ 'División Paleontología de Vertebrados, Museo de La Plata, Facultad de Ciencias Naturales y Museo, Universidad Nacional de La Plata. Paseo \\ del Bosque s/n, B1900FWA La Plata, Buenos Aires, Argentina.acostacaro@fcnym.unlp.edu.ar; agustinruella@hotmail.com; \\ nicobauza_carp@hotmail.com \\ ${ }^{2}$ Instituto de Investigaciones en Biodiversidad y Medioambiente (INIBIOMA), Consejo Nacional de Investigaciones Científicas y Tecnoló- \\ gicas-Universidad Nacional del Comahue (CONICET-UNCO). Quintral 1250, R8400FRF San Carlos de Bariloche, Río Negro, Argentina. \\ premjisaurus@yahoo.com.ar
}

Dieciséis especies de pingüinos se han descripto para el Paleógeno de la Isla Marambio (Antártida), la mayor parte de ellas conocidas exclusivamente a partir del tarsometatarso. Lamentablemente, todos los cráneos previamente hallados en esta isla carecen de elementos diagnósticos para una más acertada asignación sistemática. El ejemplar MLP 14-XI-27-84, recientemente hallado en niveles priabonianos de la Formación Submeseta, constituye el primer cráneo que fehacientemente puede ser referido a una especie antártica, siendo hallado en asociación a sus tarsometatarsos, permitiendo su asignación a Anthropornis grandis. El cráneo se encuentra altamente deformado. Posee la mandíbula casi completa, si bien carece del rostro y paladar. Otros elementos asociados incluyen fragmentos del pterigoides, costillas (algunas con procesos uncinados), vértebras cervicales y torácicas, pigostilo, y falanges pedales. A partir del escaneo tridimensional del cráneo se realizó una retrodeformación empleando el software Landmark Editor, mientras que a partir de una microtomografía computada se realizó la reconstrucción digital del endocráneo, permitieron analizar por primera vez en detalle la anatomía craneana y endocraneana (encéfalo y oído interno derecho parciales) de Anthropornis grandis. Comparativamente con las especies modernas, Anthropornis tenía un pico extremadamente elongado y angosto, y un neurocráneo pequeño con un cóndilo occipital y un foramen magnum grandes y robustos. Tanto el molde endocraneano como el oído interno muestran similitud con los pingüinos del grupo corona, como los wulst bien desarrollados y el tamaño y disposición relativa de los tres canales semicirculares (caracterizados por un canal anterior oval y marcadamente mayor que los demás).

*Proyecto financiado por PICT 2016-0481. 


\title{
CARACTERIZACIÓN FAUNÍSTICA Y PALEOAMBIENTAL DE LA FORMACIÓN LAGO COLHUÉ HUAPI (CONIACIANO-MAASTRICHTIANO) EN AFLORAMIENTOS UBICADOS EN EL SECTOR ORIENTAL DEL LAGO HOMÓNIMO
}

\author{
B.N. Alvarez ${ }^{1}$, L.M. IBIRICU² y G.A. CASAL ${ }^{3 *}$ \\ ${ }^{1}$ Centro de Investigaciones y Transferencia Golfo San Jorge-Consejo Naiconal de Investigaciones Científicas y Tecnológicas (CONICET), Uni- \\ versidad Nacional de la Patagonia San Juan Bosco. Ruta Provincial n² 1, km 4, 9005 Comodoro Rivadavia, Chubut, Argentina. \\ bruno.n.alvarez@outlook.com \\ 2Instituto Patagónico de Geología y Paleontología (IPGP), Centro Nacional Patagónico (CENPAT)-Consejo Nacional de Investigaciones Cientí- \\ ficas y Tecnológicas (CONICET). Boulevard Brown 2915, U9120ACD Puerto Madryn, Chubut, Argentina. ibiricu@cenpat-conicet.gob.ar \\ ${ }^{3}$ Laboratorio de Paleontología de Vertebrados, Facultad de Ciencias Naturales y Ciencias de la Salud, Universidad Nacional de la Patagonia \\ San Juan Bosco. Ruta Provincial n¹, km 4, 9005 Comodoro Rivadavia, Chubut, Argentina.paleogac@yahoo.com.ar
}

En el sector sureste de la costa del lago Colhué Huapi, debido a fluctuaciones del nivel del mismo, afloran temporalmente islas que incluyen rocas asignadas a la sección media de la Formación Lago Colhué Huapi (Coniaciano-Maastrichtiano). Asimismo, estos niveles se exponen en los acantilados costeros orientales. Específicamente para esos niveles, se interpreta preliminarmente una edad pre-maastrichtiana, probablemente del Santoniano-Campaniano. En la presente contribución se caracteriza el paleoambiente sedimentario y la fauna de vertebrados fósiles recuperada hasta el momento, en dichos afloramientos. El paleoambiente se interpreta como sistemas multicanalizados de tipo anastomosados, de baja sinuosidad y multiepisódicos, con importantes fluctuaciones estacionales en la descarga acuosa. El registro de dinosaurios incluye al titanosaurio Aeolosaurus colhuehuapensis, materiales fragmentarios de Ornithopoda (posiblemente pertenecientes a ornitópodos elasmáridos), tres falanges ungueales manuales de megarraptoriforme y restos de abelisáuridos. Asimismo los vertebrados no dinosaurios incluyen espinas, posiblemente de la aleta dorsal de peces teleósteos, una placa dentaria de un dipnoo indet., una escápula fragmentaria y dos ejemplares muy completos de Testudinae, dos ramas mandibulares de un Mesoeucrocodylia y osteodermos aislados asignables preliminarmente al mismo grupo. Por lo tanto, es importante resaltar la diversidad de vertebrados fósiles en estos afloramientos de la Formación Lago Colhué Huapi, la cual es potencialmente comparable a la presente en formaciones contemporáneas de la Cuenca Neuquina. Finalmente, estos niveles, presentan la mayor diversidad de vertebrados para el Cretácico Superior del Grupo Chubut en el centro sur de la provincia de Chubut.

*Proyecto parcialmente financiado por PICT 2016-0459 (a LMI y GAC).

\section{SOBRE LA PRESENCIA DE MERETRIX LAMARCK (BIVALVIA: VENERIDAE) EN EL DANIANO DE PATAGONIA}

\author{
M.J. Alvarez ${ }^{1}$ y C.J. Del Río ${ }^{1}$
}

'Museo Argentino de Ciencias Naturales "Bernardino Rivadavia" (MACN)-Consejo Nacional de Investigaciones Científicas y Tecnológicas (CONICET). Av. Ángel Gallardo 470, C1405DJR Ciudad Autónoma de Buenos Aires, Argentina. maxialvarez82@gmail.com; claudiajdelrio@gmail.com

El género Meretrix (Mioceno-Reciente; océanos Índico y Pacífico Occidental) ha sido muy poco estudiado desde un punto de vista sistemático y las descripciones de las especies integrantes de este género son muchas veces confusas. Además, bajo el nombre $M$. meretrix (especie tipo) se han incluido con frecuencia formas distintas. En este contexto de incertidumbre se realizó una revisión exhaustiva de los taxones fósiles y actuales del género para evaluar las asignaciones realizadas por autores de principios del siglo XX sobre especímenes fósiles de Patagonia y Chile. Como primer resultado se ha reasignado a M. boliviana Philippi (Mioceno Temprano-Plioceno, Chile) al género Austrocallista Erdmann y Morra. Otra de las especies más conocidas de Meretrix para esta región es $M$. chalcedonica lhering, la cual si bien posee uno de los caracteres diagnósticos del género, tal como ninfa derecha con margen ornamentado por costillas verticales, presentes además en el surco que separa el diente $4 \mathrm{~b}$ de la ninfa en la valva izquierda, también presenta significativas diferencias. Entre ellas, el contorno, Iúnula, umbón, charnela y seno paleal son diferentes a los observados en Meretrix, razón por la cual se ha decidido proponer un nuevo género que incluya a la misma y a una nueva especie identificada a partir de materiales recogidos por E. Feruglio en cercanías de Puerto Visser (Formación Salamanca), y que fueran asignados por él mismo a M. chalcedonica. De esta manera se postula que Meretrix no se haya presente en el sur de América del Sur durante el Cenozoico. 


\title{
LA IMPORTANCIA CIENTÍFICA DE LAS PRIMERAS COLECCIONES DE FÓSILES ANTÁRTICOS RECOLECTADOS POR LA EXPEDICIÓN ANTÁRTICA SUECA DE 1901-1903
}

\author{
C.R. Amenabar ${ }^{1,2}$ y C. SCHWINT ${ }^{3}$ \\ 'Departamento de Ciencias Geológicas, Facultad de Ciencias Exactas y Naturales, Instituto de Estudios Andinos "Don Pablo Groeber" (IDEAN), \\ Universidad de Buenos Aires-Consejo Nacional de Investigaciones Científicas y Tecnológicas (CONICET). Intendente Güiraldes 2160, Ciudad \\ Universitaria - Pabellón II, C1428EGA Ciudad Autónoma de Buenos Aires, Argentina.amenabar@gl.fcen.uba.ar \\ ${ }^{2}$ Instituto Antártico Argentino. Avenida 25 de Mayo 1143, B1650HMK San Martín, Buenos Aires, Argentina. \\ ${ }^{3}$ Lic. en Museologia y Gestión del Patrimonio Cultural. Chalet "Long Mile", 5178 Cruz Chica, La Cumbre, Córdoba, Argentina. \\ clarischwint@gmail.com
}

En el año 1901, el geólogo sueco Otto Nordenskjöld junto a otros exploradores realizó un viaje a la Antártida, quedando accidentalmente varados durante 2 años. A fines de 1903 fueron rescatados por la Corbeta Uruguay de la Armada Argentina, al mando del Capitán Irízar. Durante su forzosa estadía en el continente antártico, estos exploradores recolectaron los primeros fósiles. Al momento del rescate muchos de los fósiles fueron llevados a Europa y hoy en día son exhibidos en importantes museos, mientras otros quedaron en la casa de la isla Cerro Nevado (al este de la Península Antártica), una cabaña prefabricada de madera donde permanecieron los exploradores durante su estadía. En las sucesivas campañas antárticas, estos fósiles fueron llevados al Instituto Antártico Argentino (IAA) formando parte de una colección histórica. Con el relevamiento del Edificio Histórico del IAA estos fósiles fueron rescatados, catalogados y resguardados fin de recuperar su valor científico y cultural. El presente trabajo tiene dos objetivos: por un lado, presentar los fósiles hallados en la casa de Cerro Nevado, analizando el impacto que provocaron los hallazgos paleontológicos para la comunidad científica a comienzos de 1900. En segundo lugar, se discuten las motivaciones personales que llevaron a Nordenskjöld a realizar el viaje a la Antártida y se describe el contexto científico-histórico de la época, destacando las teorías geológicas vigentes. También se discute el rol de los Congresos Internacionales de Geografía de Londres y Berlín desarrollados a fines del siglo XIX, en la promoción de la investigación científica en la Antártida.

\section{PRIMER REGISTRO DEL GÉNERO AUSTRALASIÁTICO PHYLLOCLADUS(FAMILIA PODOCARPACEAE) PARA SUDAMÉRICA (EOCENO TEMPRANO DE PATAGONIA)}

\author{
A. Andruchow Colombo ${ }^{1}$, P. Wilf ${ }^{2}$ y I. Escapa ${ }^{1 *}$
}

${ }^{1}$ Museo Paleontológico Egidio Feruglio, Av. Fontana 140, U9100GYO Trelew, Chubut, Argentina. aandruchow@mef.org.ar; iescapa@mef.org.ar 2Department of Geosciences, Pennsylvania State University. 201 Old Main, 16802 University Park, Pennsylvania, Estados Unidos. pwilf@psu.edu

El género Phyllocladus (Podocarpaceae) comprende plantas con hojas muy reducidas y ejes modificados (i.e., filoclados) que actúan como órganos fotosintéticos. Este género contiene cuatro especies actuales y numerosas fósiles, conocidas casi exclusivamente para Australasia (exceptuando un registro de Antártida oriental). Presentamos el primer registro fósil sudamericano para Phyllocladus, proveniente de Laguna del Hunco (LH, Eoceno Temprano, provincia del Chubut). La nueva especie muestra filoclados elongados, dispuestos espiraladamente; estomas distribuidos y orientados azarosamente, con anillos de Florin prominentes. La disposición helicoidal de los filoclados de la nueva especie patagónica contrasta con la pseudoverticilada de los Phyllocladus actuales, y es interpretada como la disposición plesiomórfica para el género. Otras características del fósil que podrían ser consideradas plesiomórficas para el grupo son la distribución y orientación aleatoria de sus estomas. En conjunto, estas características sugieren una posición basal de la especie sudamericana en el contexto evolutivo del género. La nueva especie representa el primer registro del grupo en el hemisferio occidental, extendiendo su rango geográfico y constituyendo un linaje compartido adicional entre la flora de los bosques Iluviosos australasiáticos y la paleoflora de LH. En este contexto, la desaparición de Phyllocladus -y de otras Podocarpaceae- del sur de Sudamérica es atribuida a la baja tolerancia a la sequía de dichas especies de cara al cambio climático en la región. El nuevo Phyllocladus, que constituye además el registro más antiguo del género, representa un importante punto de calibración temporal e inferencia geográfica, aportando a la comprensión de la evolución y biogeografía del grupo.

*Proyecto financiado por National Science Foundation, grant DEB-1556666. 


\title{
LA REGIÓN AUDITIVA DEL ENIGMÁTICO PROSPANIOMYS PRISCUS AMEGHINO (RODENTIA, HYSTRICOGNATHI, OCTODONTOIDEA)
}

\author{
M.E. Arnaudo ${ }^{1}$ y M. ARnal ${ }^{2}$
}

1'División Paleontología de Vertebrados, Museo de La Plata, Facultad de Ciuencias Naturales y Museo, Universidad Nacional de La Plata. Paseo del Bosque s/n, B1900FWA La Plata, Buenos Aires, Argentina. mearnaudo@gmail.com

2División Paleontología de Vertebrados, Unidades de Investigación, Anexo, Museo de La Plata, Facultad de Ciuencias Naturales y Museo, Universidad Nacional de La Plata. Av. 122 y 60, B1904 La Plata, Buenos Aires, Argentina. michoarnal@gmail.com

Prospaniomys es un Octodontoidea que vivió en el Mioceno Temprano (Aquitaniano; Edad Colhuehuapense) de Patagonia. Este taxón presenta una morfología dentaria primitiva y una región auditiva especializada (bulas timpánicas y senos epitimpánicos muy desarrollados y exposición del petroso) semejante a la que presentan algunos Octodontidae y Abrocomidae. Esta curiosa combinación de caracteres es única y su significado evolutivo e implicancias paleobiológicas se desconocen. El objetivo de este trabajo es estudiar por primera vez la anatomía interna de la región auditiva de Prospaniomys y compararla con representantes vivientes de Octodontoidea. La cavidad del oído medio es voluminosa, como en Abrocoma y Octodontomys. El oído interno presenta la cóclea con entre dos y tres vueltas y el vestíbulo es semejante a Abrocoma (en ambos el canal coclear es robusto, parejo y no se afina hacia el ápice); el ángulo formado entre la cóclea y la región vestibular es similar a Proechimys; la ventana oval es pequeña como en Proechimys, Octodontomys y Myocastor, mientras que la ventana redonda es similar a Proechimys; los canales semicirculares son semejantes a Octodontomys y Myocastor y todos presentan el mismo grosor, a diferencia de Abrocoma. El canal lateral es diferente al de las demás especies estudiadas. Los resultados indican que Prospaniomys tiene una cavidad auditiva con caracteres presentes en especies de hábitos variados. El gran tamaño de la bula indica una adaptación para captar sonidos de bajas frecuencias. Sin embargo, el significado evolutivo de los caracteres del oído interno no es conocido y seguirá siendo estudiado.

\section{ANÁLISIS MICROPALEONTOLÓGICO DEL MIEMBRO CANAL 18 (FORMACIÓN LAS ESCOBAS), BAHÍA SAMBOROMBÓN, ARGENTINA}

\author{
A.M. Ballesteros Prada ${ }^{1}$, M. Luengo², E. Bernasconi ${ }^{1}$, I. Vilanova ${ }^{3}$ y E. Fucks ${ }^{2 *}$ \\ ${ }^{1}$ Instituto de Investigaciones en Biodiversidad y Medioambiente (INIBIOMA), Consejo Nacional de Investigaciones Científicas y Tecnológicas- \\ Universidad Nacional del Comahue (CONICET-UNCO). Quintral 1250, R8400FRF San Carlos de Bariloche, Río Negro, Argentina. \\ amballesterosprada@comahue-conicet.gob.ar; emibernasconi@yahoo.com \\ ${ }^{2}$ Facultad de Ciencias Naturales y Museo, Universidad Nacional de La Plata. Calle 64 y 120 s/n, B1904DZA La Plata, Buenos Aires, Argentina. \\ marielluengo_22@hotmail.com; efucks@fcnym.unlp.edu.ar \\ ${ }^{3}$ Museo Argentino de Ciencias Naturales "Bernardino Rivadavia" (MACN)-Consejo Nacional de Investigaciones Científicas y Tecnológicas \\ (CONICET). Av. Ángel Gallardo 470, C1405DJR Ciudad Autónoma de Buenos Aires, Argentina. ivilanova@macn.gov.ar
}

Este trabajo tiene como objetivo comprender mejor las variaciones en el nivel del mar ocurridas durante el Holoceno, en la zona norte del margen continental argentino. Para ello, se presenta el análisis de los foraminíferos bentónicos procedentes de una sucesión sedimentaria ubicada en el margen del Canal 15 (Bahía de Samborombón). La misma pertenece al miembro Canal 18 (Formación Las Escobas) y tiene 258 cm de longitud. Se determinó una edad radiocarbónica de $5900 \pm 100$ años AP en el nivel $252 \mathrm{~cm}$ y de $5550 \pm 120$ años AP en el nivel $128 \mathrm{~cm}$. La fauna de foraminíferos estudiada es relativamente abundante (aproximadamente 100 y 300 ind/gr), aunque poco diversa ( $\leq 25$ especies). Se identificaron un total de 16 géneros, principalmente correspondientes al Orden Rotaliida predominando las formas hialinas y epifaunales. Se hallaron en mayor abundancia individuos de Elphidium aff. poeyanum d'Orbigny, Buccella peruviana Boltovskoy, Ammonia tepida Cushman, Ammonia parkinsoniana d'Orbigny, Elphidium gunteri Cole, Quinqueloculina seminulina (Lineé) y Buliminella elegantissima (d'Orbigny). La abundancia y la diversidad presentaron variaciones a lo largo de la sucesión registrándose los valores más altos en los niveles medios y disminuyendo notablemente hacia el tope del perfil. Estas variaciones estarían sugiriendo una transición hacia un ambiente marino marginal con condiciones estuarinas. Los cambios en la abundancia y la diversidad reflejados en el reemplazo de especies, podría deberse a variaciones en el contenido salino.

*Proyecto financiado por Agencia de Promoción Científica y Tecnológica (PICT 2014-1271). 


\title{
PRELIMINARY REVISION OF THE CINGULATA DASYPODIDAE AND PELTEPHILIDAE FROM THE CLASSICAL SITE ARROYO CHASICÓ (LATE MIOCENE), BUENOS AIRES PROVINCE, ARGENTINA
}

\author{
D. Barasoain ${ }^{1}$, R.L. TOMASSINI ${ }^{2}$, A.E. Zurita ${ }^{1}$, And C.I. Montalvo ${ }^{3}$
}

\begin{abstract}
${ }^{1}$ Centro de Ecología Aplicada del Litoral (CECOAL)-Consejo Nacional de Investigaciones Científicas y Tecnológicas (CONICET) y Universidad Nacional del Nordeste. Ruta 5, km 2,5, 3400 Corrientes, Argentina.danielbarasoain@gmail.com; aezurita74@yahoo.com.ar

2Instituto Geológico del Sur (INGEOSUR)-Consejo Nacional de Investigaciones Científicas y Tecnológicas (CONICET), Departamento de Geología, Universidad Nacional del Sur (UNS). Av. Alem 1253, B8000CPB Bahía Blanca, Buenos Aires, Argentina. rodrigo.tomassini@yahoo.com.ar ${ }^{3}$ Facultad de Ciencias Exactas y Naturales, Universidad Nacional de La Pampa, Uruguay 151, L6300CLB Santa Rosa, La Pampa, Argentina. cmontalvolp@yahoo.com.ar
\end{abstract}

The classical fossiliferous site Arroyo Chasicó constitutes the type locality of the Chasicoan Stage/Age (Late Miocene), a key period for understanding the evolutionary history of many mammal clades, including the Cingulata, because at that time there was an important faunistic replacement with the appearance of new taxa. It includes the most ancient fauna of the Pampean Region. In this context, a taxonomic review with modern criteria has been done for the Chasicoan cingulates. A preliminary revision of Dasypodidae and Peltephilidae was carried out based on new collections with precise geographic and stratigraphic data recovered from deposits of the Arroyo Chasicó Formation, outcropping in the site. The analysis allowed us to confirm the presence of the Eutatini Chasicotatus ameghinoi Scillato-Yané, the Euphractini Vetelia perforata ScillatoYané, and the Peltephilidae Epipeltephilus kantii González-Ruiz. We added the first record of the Euphractini Proeuphractus limpidus Ameghino, Chorobates Reig, and Macrochorobates Scillato-Yané. Also, three different morphotypes (two Euphractini gen. et sp. indet. and one Eutatini gen. et sp. indet.) were identified; preliminary observations of their anatomical characters suggest that they could represent new taxa. Our results highlight that the Chasicoan was a period of high diversity for Dasypodidae, with a well representation of the tribes Euphractini and Eutatini. This fauna shows clear similarities with other Late Miocene (Chasicoan and Huayquerian) assemblages recovered from levels of the Cerro Azul Formation, outcropping in La Pampa and Buenos Aires provinces.

\section{PRIMER REGISTRO DE ASTRAPOTHERIIDAE (MAMMALIA, ASTRAPOTHERIA) DE LA FORMACIÓN CULLEN (MIOCENO TEMPRANO) DE TIERRA DEL FUEGO}

\author{
M.S. Bargo ${ }^{1}$, E. Cerdeño², E.B. Olivero 3 , M.I. López Cabrera ${ }^{3}$, M.A. Reguero ${ }^{4}$ y S.F. Vizcaíno ${ }^{1 *}$
}

1División Paleontología de Vertebrados, Unidades de Investigación Anexo Museo, Facultad de Ciencias Naturales y Museo, Universidad Nacional de La Plata. Av. 60 y 122, B1904 La Plata, Buenos Aires, Argentina.msbargo@fcnym.unlp.edu.ar; vizcaino@fcnym.unlp.edu.ar ${ }^{2}$ Paleontología, Instituto Argentino de Nivología, Glaciología y Ciencias Ambientales (IANIGLA), Centro Científico Tecnológico MendozaConsejo Nacional de Investigaciones Científicas y Tecnológicas (CONICET). Avenida Ruiz Leal s/n, 5500 Mendoza, Mendoza, Argentina. espe@mendoza-conicet.gob.ar

${ }^{3}$ Laboratorio de Geología Andina, Centro Austral de Investigaciones Científicas (CADIC)-Consejo Nacional de Investigaciones Científicas y Técnicas (CONICET). Bernardo A. Houssay 200, V9410CAB Ushuaia, Tierra del Fuego, Argentina. emolivero@gmail.com

${ }^{4}$ División Paleontología Vertebrados, Museo de La Plata, Facultad de Ciencias Naturales y Museo, Universidad Nacional de La Plata. Paseo del Bosque s/n, B1900FWA, La Plata, Buenos Aires, Argentina. regui@fcnym.unlp.edu.ar

En los acantilados de la costa Atlántica del NO de Tierra del Fuego, entre el Cañadón Alfa y el cabo Espíritu Santo, se exponen cerca de 40 m de depósitos tobáceos fluviales del Mioceno, que localmente han sido referidos a la Formación Cullen y correlacionados con la Formación Santa Cruz, expuesta unos 140 km hacia el N en la provincia de Santa Cruz. En cuanto al contenido fósil, se han descripto en paleosuelos álficos trazas fósiles asignables a escarabajos peloteros y el mamífero Nesodon (Notoungulata). En este trabajo se da a conocer el primer registro de un astrapoterio (Mammalia, Astrapotheria, Astrapotheriidae), procedente de un horizonte de tufita con estratificación entrecruzada de gran escala, que en su parte superior preserva restos de raíces carbonizadas in situ. El espécimen (CADIC P 607) es un M2 derecho poco gastado, atribuible a un adulto joven. El escaso desarrollo del cíngulo anterolingual y la altura de la corona lo diferencian de Astrapothericulus (edades Colhuehuapense, Mioceno Temprano, Neuquén; "Astrapothericulense", "Notohipidense" y Santacrucense, Mioceno Temprano, Santa Cruz), permitiendo su asignación al género Astrapotherium, típico de la Edad Santacrucense. Si bien la morfología oclusal de los M1- 2 es similar, la fuerte proyección labial del pliegue paracono es más propia del M2; las dimensiones de CADIC P 607 resultan pequeñas para un M2 adulto de Astrapotherium magnum, pero tanto la talla como el contorno oclusal varían con la ontogenia.

*Proyecto financiado por PICT -2013 0389, UNLP N/750. 


\title{
HUELLAS DE UN CRIMEN: OICHNUS BROMLEY COMO EVIDENCIA DE PREDACIÓN EN EL ÁRTICO CANADIENSE
}

\author{
M.S. BAYER ${ }^{1}$, D.F. Muñoz ${ }^{1}$ y A.E. AITKEn²* \\ ${ }^{1}$ Centro de investigaciones en Ciencias de la Tierra (CICTERRA)-Consejo Nacional de Investigaciones Científicas y Técnicas (CONICET), Universi- \\ dad Nacional de Córdoba. Av. Vélez Sársfield 1611,X5016CGA Córdoba, Córdoba, Argentina.sol.bayer@conicet.gov.ar; df.munoz@conicet.gov.ar \\ 2Deptartment of Geography and Planning, University of Saskatchewan. 117 Science Place, S7N 5C8 Saskatoon, Saskatchewan, Canada. \\ alec.aitken@usask.ca
}

En el Cuaternario tardío marino del Ártico canadiense, los moluscos son los fósiles más comunes. En la isla Axel Heiberg (AH) las interacciones predador-presa son poco conocidas debido a la baja preservación de moluscos predadores, y estas interacciones se evidencian por perforaciones en valvas que se asignan al icnogénero Oichnus Bromley. Los principales sospechosos de producir estas trazas en bivalvos son gastrópodos, aunque algunos octópodos han sido sugeridos. Nuestro objetivo es identificar icnoespecies de Oichnus en bivalvos como evidencia de interacción biótica revelando la posible presión de predación en bivalvos durante el Holoceno en AH. De un total de 3551 valvas solo el 0,48\% presentan perforaciones completas y el 0,73\% incompletas. Estas perforaciones se encuentran principalmente en la región umbonal de bivalvos infaunales. Se identificaron cuatro icnoespecies: Oichnus simplex Bromley, Oichnus ovalis Bromley, Oichnus paraboloides Bromley y Oichnus excavatus Donovan y Jagt. Oichnus simplex es comúnmente atribuido a gastrópodos murícidos, mientras 0 . paraboloides a gastrópodos natícidos. Oichnus ovalis puede ser producido por octópodos. Las marcas de predación fallidas (O. excavatus) se atribuyen comúnmente a gastrópodos natícidos. En este contexto, la presencia de Oichnus sugiere que al menos los representantes de las familias Naticidae y Muricidae habrían estado presentes en las comunidades bentónicas fósiles, sin descartar la presencia de otros predadores, como octópodos. La baja proporción de Oichnus en AH demuestra una baja presión de predación y su diversidad permitirá comprender las diferentes estrategias de predación en bivalvos del Ártico canadiense durante el Cuaternario tardío.

*Proyecto financiado por la University of Saskatchewan, Canadá.

\section{STABLE OXYGEN ISOTOPE ANALYSIS IN AMIANTIS PURPURATA SHELLS (BIVALVIA, VENERIDAE): FIRST INDICATIONS FOR LATE HOLOCENE CLIMATE VARIABILITY IN NORTHERN PATAGONIA, ARGENTINA}

\author{
M.S. Bayer ${ }^{1}$, L. Beierlein², T. Brey², L. Farías ${ }^{3}$, G. García³ , E. Morsan ${ }^{4}$, and S. Gordillo ${ }^{1 *}$
}

${ }^{1}$ Centro de investigaciones en Ciencias de la Tierra (CICTERRA)-Consejo Nacional de Investigaciones Científicas y Técnicas (CONICET)- Universidad Nacional de Córdoba. Av. Vélez Sársfield 1611,X5016CGA Córdoba, Córdoba, Argentina. sol.bayer@conicet.gov.ar; sandra.gordillo@unc.edu.ar ${ }^{2}$ Alfred Wegener Institute, Helmholtz Centre for Polar and Marine Research. Am Handelshafen 12, D-27570, Bremerhaven, Bremen, Alemania. lars.beierlein@awi.de; thomas.brey@awi.de

${ }^{3}$ Departamento de Oceanografia, Universidad de Concepción, Centro de Ciencia del Clima y la Resiliencia (CR2). Barrio Universitario s/n, Casilla 160-C,4030000 Concepción, Concepción, Chile. laura.farias@udec.cl; gegarcia@udec.cl

"Centro de Investigación Aplicada y Transferencia Tecnológica en Recursos Marinos "Almirante Storni", Consejo Nacional de Investigaciones Científicas y Técnicas (CONICET)-Universidad Nacional del Comahue. Güemes 1030, R8520CXV San Antonio Oeste, Río Negro, Argentina. qmorsan@gmail.com

The shells of several mollusks can provide environmental and climatic information on a seasonal scale. This variability is recorded within the stable oxygen isotope ( $\delta 180$ shell) composition of the biogenic shell carbonate. The $\delta 180$ shell values depend on the composition of the sea water ( $\delta 180$ water) and changes in water temperature. The bivalve Amiantis purpurata Lamarck has a well-preserved fossil record from the Late Holocene to the present times in the San Matías Gulf (SMG). The goal of this study is to detect the seasonal ( $\delta 180$ shell) signals of $A$. purpurata in order to reconstruct the seasonal water temperatures within the SMG during the Late Holocene. Seven shells were radiocarbon dated (14CAMS), each representing a different time horizon throughout the Late Holocene, up to the present. The intra-annual variability of $\delta 180$ shell values was largest in the modern shell $(\Delta \delta 180$ shell= $2.23 \%$ ), while the fossil shells (3844-3555 yrs BP) provided the smallest intra-annual amplitude $(\Delta \delta 180$ shell= $1.39 \%$ ). $\delta 180$ shell values in modern and fossil shells were used to calculate water temperatures based on modern $\delta 180$ water measurements, which have not been reported in SMG so far. In general, the reconstructed temperatures fit well into the general assumptions for the coldest and warmest phases throughout the Holocene in SMG. However, we are aware that this study represents a first step towards the calibration of $A$. purpurata as a new palaeo archive for the Southern Hemisphere and into the climatic history of SMG and Northern Patagonia.

*Financial support by the Scientific and Technological Research Fund, FONCYT (S.B., grant number PICT 2016-2951), LF and GG CONICYT/ FONDAP program no. 1511009. 


\title{
BAJO DE VÉLIZ STROMATOLITES REVISITED
}

\author{
C.A. Benavente ${ }^{1}$, A. Mancuso ${ }^{1}$ y E. Crespo ${ }^{2}$
}

${ }^{1}$ Instituto Argentino de Nivología, Glaciología y Ciencias Ambientales (IANIGLA), Centro Científico Tecnológico Mendoza-Consejo Nacional de Investigaciones Científicas y Tecnológicas (CONICET). Avenida Ruiz Leal s/n, 5500 Mendoza, Mendoza, Argentina. cbenavente@mendoza-conicet.gob.ar; amancu@gmail.com

'Laboratorio de Microscopía Electrónica y Microanálisis (LabMEM), Universidad Nacional de San Luis. Av. Ejército de los Andes, Bloque II, 5700 San Luis, San Luis, Argentina. estebancrespo0@gmail.com

The discussion about the true biogenicity of Ca-rich deposits has been extensive, and in the past, abiogenic structures have been referred to stromatolites when their origin was linked to inorganic precipitation of carbonate. Later on, different types of carbonate precipitation have been described including: mineralization (inorganic); and biogenic precipitation of carbonates, namely: biologically controlled, biologically induced and biologically influenced mineralization. The Bajo de Véliz subbasin, located on the east margin of the Paganzo basin, in the NE of San Luis province, presents the Bajo de Véliz Formation, referred to as Permian. The unit is a siliciclastic succession, well known by its extraordinary fossiliferous content and includes carbonate levels. The carbonates have been previously described as stromatolites based on microfacies analysis with the identification of bryophyte and cyanobacteria as producers. Nevertheless, new polished sections and thin section microfacies analysis under low magnification binocular microscope along with SEM-EDS analysis shows no trace of biogenicity at a microstructure level. New data shows that the microfabric is formed by a combination of Ca-rich parallel and subparallel veins (spar), included in a Si-rich matrix with Fe content. These microstructures were previously identified as bryophyte remains but no feature other than morphological resemblance was found. This point to a genesis linked to a probable carbonate rich seep that caused Ca and Si mineralization in the form of complex dendrites. Further cathodoluminescence analysis can confirm the timing and different phases of mineralization and if there was incremental growth.

\section{HETEROCHRONY, A PERVASIVE MECHANISM IN GENERATING EVOLUTIONARY TRENDS IN BRACHIOPODS: SOME EXAMPLES FROM ORDOVICIAN AND SILURIAN RHYNCHONELLIFORMS}

\section{J.L. Benedetto ${ }^{1}$}

${ }^{1}$ Centro de investigaciones en Ciencias de la Tierra (CICTERRA)-Consejo Nacional de Investigaciones Científicas y Técnicas (CONICET) and Universidad Nacional de Córdoba. Av. Vélez Sársfield 1611, X5016CGA Córdoba, Córdoba, Argentina. juan.benedetto@unc.edu.ar

Evolutionary lineages preserved in the fossil record are critical to interpreting evolutionary processes (e.g., gradual vs. punctuated phyletic change) but interpretation of the underlying mechanisms is challenging. Among the many causes invoked to produce evolutionary trends - defined as a persistent directional change in one or more character states through timeheterochrony has been recognized as a crucial factor not only as an agent in speciation but also in macroevolution. All of the Ordovician successions of Argentina (NW basin, Famatina Range, Cuyania terrane) provided well documented examples of evolutionary trends in orthoid, plectorthoid and plectambonitoid brachiopods. Such trends involve size, shell morphology, ornamentation and, more importantly, changes in the development of such internal structures as cardinalia, which are often considered to be 'stable' and thus taxonomically (and phylogenetically) informative. The lineage found in the Santa Victoria Group leading from the basal plectorthoid Protorthisina (Furongian) to the lower to upper Tremadocian 'nanorthids' Kvania, Gondwanorthis, Lampazarorthis and Tarfaya is a good example of a long-term trend (ca. 10 Ma) directed mainly by heterochronic processes, but perhaps the best documented hypothesis of heterochrony (peramorphosis) is the PanderinaProductorthis lineage recorded in the Floian-Dapingian Famatina Group. There is also compelling evidence supporting that the Silurian rhynchonellide Clarkeia (Uncinuloidea) evolved from Harringtonina (Rhynchotrematoidea) by peramorphosis. More striking is the case of the plectambonitoid Ahtiella famatiniana (Strophomenata), which on the basis of empirical and cladistic evidence evolved from the orthoid Monorthis transversa (Rhynchonellata) showing that in brachiopods heterochrony may have played a substantial role in originating higher taxa (macroevolution). 


\title{
DIGITALIZACIÓN Y PUESTA EN VALOR DE LA COLECCIÓN DE LA DIVISIÓN CIENTÍFICA DE GEOLOGÍA, MUSEO DE LA PLATA: RESULTADOS PRELIMINARES
}

\author{
A. Bidone 1 , S. Apcarian ${ }^{1}$, M. Chaumeil Rodríguez ${ }^{1,2}$, M. Escalona ${ }^{1,3}$, J. Omar ${ }^{1}$ y A. Siccardi, ${ }^{1,4 *}$ \\ ${ }^{1}$ Cátedra de Geología Histórica, Facultad de Ciencias Naturales y Museo, Universidad Nacional de La Plata. Calle 120 y 60 s/n, 1900 La Plata, \\ Buenos Aires, Argentina.andreabidone@yahoo.com.ar; sofiaapcarian@yahoo.com.ar; omarjesica@gmail.com \\ 2Laboratorio de Bioestratigrafía, YPF Tecnología S.A.-Consejo Nacional de Investigaciones Científicas y Tecnológicas (CONICET). Avenida del \\ Petróleo Argentino s/n entre 129 y 143, 1923 Berisso, Buenos Aires, Argentina.micaela.chaumeil@ypftecnologia.com \\ 32División Científica de Geología, Museo de La Plata, Facultad de Ciencias Naturales y Museo de La Plata, Universidad Nacional de La Plata. \\ Paseo del Bosque s/n, B1900FWA La Plata, Buenos Aires, Argentina. escalonamaxi@gmail.com \\ ${ }^{4}$ Centro de Investigaciones Geológicas (CIG), Universidad Nacional de La Plata-Consejo Nacional de Investigaciones Científicas y Tecnológicas \\ (CONICET). Diagonal 113 n² 275, B1904DPK La Plata, Buenos Aires, Argentina.aron@fcnym.unlp.edu.ar
}

La División Científica de Geología (DCG) del Museo de La Plata alberga una importante colección de fósiles y rocas de diversas localidades, especialmente argentinas, producto de numerosos viajes de campaña realizados por miembros de la DCG -investigadores como Schiller, Seilacher, Borrello, Cuerda, Schauer y Cingolani, entre otros- además de donaciones de otras instituciones y particulares. Durante aproximadamente dos años se han contabilizado más de 12.500 muestras, cuya puesta en valor incluyó control del inventario, limpieza, numeración y fotografía del material; digitalización de las etiquetas preexistentes, y confección de nuevas en caso de ser necesario por ausencia o deterioro; y reordenamiento. En aquellos casos en los que el material no se encontrara debidamente identificado, se lo analizó con el objetivo de sistematizarlo; particularmente para los fósiles mediante métodos no destructivos, en tanto que con las rocas se utilizó una mínima porción, salvaguardando casi la totalidad de la muestra y dejando constancia del proceso ejecutado. La actualización y restructuración del registro de todo el material surgió como parte de las labores docentes y de investigación de quienes participan en el presente trabajo, ya que el mismo puede utilizarse tanto en el aula como en proyectos de extensión, enseñanza, elaboración de tesinas y trabajos de investigación. El objetivo final es la publicación de un registro on-line georreferenciado, adecuándose a la tendencia de varias instituciones nacionales e internacionales que promueven el acceso a la información de sus colecciones, respetando la reglamentación vigente en el Museo de La Plata para el manejo de sus colecciones.

*Proyecto financiado por PPID, UNLP. Caracterización estratigráfica y procedencia de las secuencias pre-carboníferas del suroeste del Macizo Nordpatagónico. Implicancias paleogeográficas.

\section{LOS NEOCETI (MAMMALIA: CETACEA) DEL MIOCENO DE PATAGONIA: NUEVOS APORTES SOBRE SU DIVERSIDAD Y PALEOBIOLOGÍA}

\author{
M. Buono ${ }^{1}$, M. Viglino ${ }^{1}$, F. Paolucci², C.M. Gaetán ${ }^{3}$, L. Alzugaray ${ }^{1}$, J.I. Cuitiño ${ }^{1}$ y M.S. Fernández ${ }^{2 *}$
}

${ }^{1}$ Instituto Patagónico de Geología y Paleontología (IPGP), Centro Nacional Patagónico (CENPAT)-Consejo Nacional de Investigaciones Científicas y Tecnológicas (CONICET). Boulevard Brown 2915, U9120ACD Puerto Madryn, Chubut, Argentina. buono@cenpat-conicet.gob.ar; viglino@cenpatconicet.gob.ar; lualzugaray@hotmail.com; jcuitino@cenpat-conicet.gob.ar

2División Paleontología de Vertebrados, Unidades de Investigación Anexo Museo, Facultad de Ciencias Naturales y Museo, Universidad Nacional de La Plata. Av. 60 y 122 s/n, B1904 La Plata, Buenos Aires, Argentina.paolucciflorencia@fcnym.unlp.edu.ar; martafer@fcnym.unlp.edu.ar 33maxgaetan88@gmail.com

El registro de Neoceti miocenos de Patagonia es particularmente relevante ya que incluye taxones claves que completan hiatos estratigráficos y geográficos, y que contribuyen al entendimiento de la historia evolutiva más temprana de misticetos y odontocetos. Gran parte de los taxones conocidos fueron descriptos en el siglo XIX y XX, y permanecieron durante mucho tiempo como casos enigmáticos dentro de la comunidad científica, ya que no habían sido re-estudiados. En los últimos años se re-estudiaron tanto especies históricas como nuevos ejemplares recolectados, en el marco de un estudio sistemático y paleobiológico moderno. Durante el Mioceno en Patagonia, los cetáceos estaban representados por misticetos balénidos, cetotéridos y balaenopteroideos, y odontocetos platanistoideos y fiseteroideos. El relevamiento de la diversidad primaria sugiere un recambio faunístico hacia el Mioceno Tardío, que implicó la extinción de algunas formas (e.g... cetotéridos y platanistoideos) y la diversificación de los linajes modernos (e.g., fiseteroideos, balenoptéridos y balénidos). Los estudios paleobiológicos sugieren que durante el Mioceno la comunidad de cetáceos estaba representada por diferentes morfotipos (e.g., diversidad de tamaño corporales y de estructuras cráneomandibulares y dentarias), que estarían vinculados a la explotación de diferentes nichos ecológicos. Finalmente, la integración de estos datos en un contexto filogenético permitió identificar innovaciones claves asociadas con la diversificación de algunos de los grupos (e.g., retención de la conexión ósea de los huesos del oído con el cráneo en platanistoideos; alimentación por filtración pasiva y audición de bajas frecuencias en balénidos).

*Proyecto fianciado por PICT 2015-0792; SAREM; Society Marine Mammalogy; Cetacean Society International. 


\title{
CAMINANDO SOBRE LO CAMINADO, UN NUEVO ROEDOR (HYSTRICOGNATHI, CHINCHILLOIDEA) DE LA LOCALIDAD DE BRYN GWYN (MIOCENO TEMPRANO, PATAGONIA, ARGENTINA)
}

\author{
F. BUSKer ${ }^{1}$, M.E. PÉrez ${ }^{2}$ y M.T. Dozo ${ }^{1}$
}

${ }^{1}$ Instituto Patagónico de Geología y Paleontología (IPGP), Centro Nacional Patagónico (CENPAT)-Consejo Nacional de Investigaciones Científicas y Tecnológicas (CONICET). Boulevard Brown 2915, U9120ACD Puerto Madryn, Chubut, Argentina. busker@cenpat-conicet.gob.ar; dozo@cenpat-conicet.gob.ar

${ }^{2}$ Museo Paleontológico Egidio Feruglio. Av. Fontana 140, U9100GYO Trelew, Chubut, Argentina. perezmariae@gmail.com

Bryn Gwyn (Chubut) es una de las localidades más prospectadas y ricas en roedores fósiles de la Patagonia y, junto a Gran Barranca (Chubut), han brindado la mayor parte del registro conocido para la Edad Mamífero Colhuehuapense (Mioceno Temprano). En este contexto presentamos un nuevo chinchilloideo, sobre la base de restos hallados en Bryn Gwyn, provenientes del Miembro Trelew, Formación Sarmiento. Estos corresponden a mandíbulas fragmentarias y dientes aislados, depositados en el Museo Paleontológico Egidio Feruglio (MPEF). A este nuevo taxón se suma un molar superior aislado proveniente de niveles colhuehuapenses de Gran Barranca, descripto previamente como Chinchilloidea incertae sedis. La asignación a un nuevo taxón se basa en los siguientes caracteres: molares protohipsodontes, banda de esmalte completa y continua, ausencia de cemento, patrón trilofodonte en molares superiores y tetralofodonte en inferiores, segunda cresta en posición de desarrollo variable en molares y premolares inferiores, dp4 pentalofodonte con neomesolófido, dos o tres fosétidas persistentes, foramen mentoniano dorsolabial y raíz del incisivo extendiéndose hasta el m3. Este conjunto de caracteres lo diferencian de otros chinchilloideos protohipsodontes conocidos (e.g., Incamys, Scleromys, Garridomys). Este nuevo taxón, el segundo Chinchilloidea conocido para Bryn Gwyn, corresponde a uno de los pocos géneros compartidos con Gran Barranca y amplía la diversidad sistemática de este grupo para el Mioceno Temprano de la Patagonia, donde su registro fósil es relativamente pobre. Además, este es un momento clave de su historia evolutiva, previo al reemplazo de taxones protohipsodontes con morfologías dentarias más complejas a formas euhipsodontes de superficie oclusal simplificada.

\section{NUEVOS HALLAZGOS DE FORAMINÍFEROS BENTÓNICOS EN EL MIEMBRO LA TOSCA DE LA FORMACIÓN HUITRÍN (BARREMIANO, CUENCA NEUQUINA) EN EL SUR DE MENDOZA Y SU SIGNIFICADO PALEOAMBIENTAL}

\author{
A. Caramés ${ }^{1}$, C.S. Cataldo ${ }^{1}$, D.G. Lazo ${ }^{1}$, L. Luci ${ }^{1}$, M. Lescano ${ }^{1}$ y M.B. Aguirre-Urreta ${ }^{1 *}$
}

1Departamento de Ciencias Geológicas, Facultad de Ciencias Exactas y Naturales, Instituto de Estudios Andinos "Don Pablo Groeber" (IDEAN), Universidad de Buenos Aires-Consejo Nacional de Investigaciones Científicas y Tecnológicas (CONICET). Intendente Güiraldes 2160, Ciudad Universitaria - Pabellón II, C1428EGA Ciudad Autónoma de Buenos Aires, Argentina.carames@gl.fcen.uba.ar; ceciliacataldo@gl.fcen.uba.ar; dlazo@gl.fcen.uba.ar; leticialuci@gl.fcen.uba.ar; lescano@gl.fcen.uba.ar; aguirre@gl.fcen.uba.ar

Se da a conocer una asociación relativamente abundante y de baja diversidad de foraminíferos bentónicos recuperada de un nivel de margas yesosas friables finamente laminadas del Miembro La Tosca (Formación Huitrín) en la localidad de Quebrada del Gastrópodo, Sierra de Cara Cura, sur de Mendoza. La edad de la misma no podría ser más joven que barremiana temprana por la presencia de la especie de nanofósil Diloma galiciense. La asociación de foraminíferos está conformada por al menos ocho taxones con un grado de preservación pobre, sobre todo debido a procesos de disolución y deformación. Patellina subcretacea Cushman y Alexander es el elemento más abundante de esta fauna, seguido por Eoguttulina anglica Cushman y Ozawa, Polymorphinidae indeterminados y Guttulina sp. La asociación se completa con escasos ejemplares de Quinqueloculina sp., Lingulina sp. cf. loryi (Berthelin), ?Hergotella sp., Lingulina sp. y ?Laevidentalina sp. Muchos de estos registros coinciden o son comparables a otros de la subyacente Formación Agrio marcando una cierta continuidad en el registro de foraminíferos entre ambas unidades. Si bien una interpretación paleoambiental podría estar sesgada tafonómicamente, la baja diversidad de la asociación y la abundancia del género Eoguttulina indicarían un ambiente marino marginal somero sujeto a estrés, posiblemente por variaciones de salinidad. Esta interpretación, si bien preliminar, es consistente con la sucesión de facies sedimentarias registradas y la presencia de asociaciones de bivalvos, gastrópodos y serpúlidos de baja diversidad y alta abundancia en la misma localidad.

*Proyecto financiado por Contribución C-153 del Instituto de Estudios Andinos "Don Pablo Groeber". 


\title{
COROLARIO DE CURSOS DE LIMPIEZA Y PREPARACIÓN BÁSICA DE FÓSILES Y CONFECCIÓN DE MOLDES EN EL MUSEO PALEONTOLÓGICO BARILOCHE
}

\author{
M. Cárdenas ${ }^{1}$, A. Iglesias² y A. Paulina Carabajal ${ }^{2}$
}

${ }^{1}$ Instituto de Investigación en Paleobiología y Geología (IIPG)-Consejo Nacional de Investigaciones Científicas y Tecnológicas (CONICET), Universidad Nacional de Río Negro. Av. Roca 1242, R8332EXZ Gral. Roca, Río Negro, Argentina. mcardenas@unrn.edu.ar

2Instituto de Investigaciones en Biodiversidad y Medioambiente (INIBIOMA), Consejo Nacional de Investigaciones Científicas y TecnológicasUniversidad Nacional del Comahue (CONICET-UNCO). Quintral 1250, R8400FRF San Carlos de Bariloche, Río Negro, Argentina.

ari_iglesias@yahoo.com.ar; premjisaurus@yahoo.com.ar

La Asociación Paleontológica Bariloche vio la necesidad de capacitar a sus socios y colaboradores del Museo y generó (período 2015-2018) siete cursos de preparación de fósiles de nivel inicial, dictados por técnicos en paleontología, autofinanciados y con bajo costo. Los mismos fueron originalmente orientados a personas aficionadas o no idóneas en temas paleontológicos, pero se formalizaron en las últimas ediciones debido al alto número de estudiantes y profesionales de la paleontología interesados en esta capacitación, siendo el alcance de la convocatoria internacional Argentina, Chile, México y Brasil) y sumando un total de 64 participantes. El espacio del laboratorio fue reducido por lo que se replicaron cursos con una duración de tres días (30 horas) cada uno. Los inscriptos incluyeron estudiantes secundarios y universitarios de carreras de biología y paleontología de diversas universidades nacionales, y también empleados de museos. Como resultado del éxito en estos cursos, otros dos se ofrecieron en Valdivia (Chile) y en General Roca, ampliando temáticas específicas según los requerimientos regionales. De la enorme demanda para esta actividad generada en una pequeña institución alejada de los centros más desarrollados, se pone en evidencia la escasez de capacitaciones de este tipo, como también la necesidad de un espacio donde intercambiar dudas y conocimientos. Se han brindado herramientas tanto teóricas como prácticas para resolver problemáticas particulares sobre fósiles de la región patagónica en instituciones que no cuentan con equipamiento o insumos tecnológicos.Varios de los cursantes locales que concurrieron al taller, aún siguen colaborando como voluntarios en el Museo.

\section{INTERACCIONES PLANTA-ARTRÓPODO EN LA FLORA PÉRMICA DE LA FORMACIÓN CARAPACHA (LA PAMPA, ARGENTINA)}

\author{
B. CARIGLINO ${ }^{1}$ Y R. MELCHOR ${ }^{2 *}$
}

${ }^{1}$ Museo Argentino de Ciencias Naturales "Bernardino Rivadavia". Av. Angel Gallardo 470, C1405DJR Ciudad Autónoma de Buenos Aires, Argentina.barichi10@gmail.com

${ }^{2}$ Instituto de Ciencias de la Tierra y Ambientales de La Pampa (INCITAP), Consejo Nacional de Investigaciones Científicas y Técnicas (CONICET)Universidad Nacional de La Pampa. Av. Uruguay 151, L6300CLB Santa Rosa, La Pampa, Argentina. melchor.ricardo@gmail.com

Se dan a conocer los primeros registros de interacciones planta-artrópodo para la flora pérmica de la Formación Carapacha, provincia de La Pampa. Los restos con daño de artrópodos proceden del Miembro Urre-Lauquén, cuya edad es Pérmico Temprano tardío a Pérmico Tardío temprano. Las facies portadoras son esencialmente de planicie de inundación fluvial y afloran en las cercanías de la localidad de Puelches. El elenco paleoflorístico consta principalmente de glossopterídeas (hojas de Gangamopteris, Glossopteris y fructificaciones), con hojas de cordaitales, helechos y tallos de esfenofitas como elementos secundarios. Las trazas se identificaron únicamente en hojas de glossopterídeas, y corresponden a daño externo foliar (por alimentación del tipo marginal, en orificio, o superficial), perforación y succión, oviposición y agallas. Si bien los datos aquí presentados son preliminares dado que las observaciones se realizaron en material de colección poco numeroso ( $n<200$ ), en la Formación Carapacha se reconocieron 9 tipos de daño ('DTs' sensu Labandeira et al.). Estos valores son similares a los registrados en floras más diversas y numerosas del Pérmico de Argentina, como ser Tepuel-Genoa en Chubut ( $n=291 ; 10$ DTs) o Bajo de Véliz en San Luis ( $n=212 ; 6$ DTs). Sin embargo, dista de los valores contabilizados para la Formación La Golondrina ( $n=2.523 ; 37$ DTs). Futuras colectas de material fósil en la zona permitirán realizar comparaciones más rigurosas, y definir si las diferencias de registros de trazas fósiles entre las paleofloras se deben exclusivamente a un sesgo de muestreo, o reflejan la influencia de factores ambientales, faciales, y/o temporales.

*Proyecto financiado por PICT 2016-0431. 


\title{
DARWINULOCOPINA (OSTRACODA) TRIÁSICOS DE LA FORMACIÓN CERRO DE LAS CABRAS, CUENCA CUYANA, MENDOZA, ARGENTINA. RESULTADOS PRELIMINARES
}

\author{
A.P. Carignano 1,3 y A.M. Zavattieri ${ }^{2 *}$
}

${ }^{1}$ Consejo Nacional de Investigaciones Científicas y Tecnológicas (CONICET). División Paleozoología de Invertebrados, Museo de La Plata, Facultad de Ciencias Naturales y Museo, Universidad Nacional de La Plata. Paseo del Bosque s/n, B1900FWA La Plata, Buenos Aires, Argentina. apcarignano@fcnym.unlp.edu.ar

${ }^{2}$ Instituto Argentino de Nivología, Glaciología y Ciencias Ambientales (IANIGLA), Centro Científico Tecnológico Mendoza-Consejo Nacional de Investigaciones Científicas y Tecnológicas (CONICET). Avenida Ruiz Leal s/n, 5500 Mendoza, Mendoza, Argentina.amz@mendoza-conicet.gob.ar

Los Darwinulocopina se encuentran entre los primeros ostrácodos que invadieron el medio acuático continental durante el Carbonífero, alcanzando su mayor diversidad durante el Pérmico. La extinción permo-triásica redujo drásticamente al grupo, que llega a la actualidad representado únicamente por la Familia Darwinulidae. Los ostrácodos triásicos de Argentina son poco conocidos y existen escasos trabajos dedicados a ellos para este periodo. Se recuperaron aproximadamente 226 caparazones de una única muestra, proveniente de la Formación Cerro de Las Cabras (Triásico Medio bajo) en su localidad y perfil tipo, área de Potrerillos, Mendoza. Los especímenes presentan las siguientes características distintivas: caparazón con forma de gota en vista dorsal y el mayor ancho en el tercio posterior; cambio en la posición del ancho máximo durante la ontogenia (de la región media hacia el tercio posterior); cubrimiento de la valva izquierda sobre la derecha, inverso en la charnela; contacto de las valvas en vista posterior desplazado del plano vertical de simetría; charnela aparentemente formada por dos elementos (barra y diente); y aproximadamente 9 cicatrices musculares. Estos caracteres coinciden marcadamente con Prasuchonella Molostovskaya (Suchonelloidea, Darwinulocopina), descripto originalmente en el Pérmico superior no marino de Rusia, y hasta el momento sin registros más jóvenes ni menciones fuera de esta región. Sin embargo, darwinulocopinos del Jurásico Temprano de Estados Unidos y de la transición permo-triásica en el este europeo, previamente asignados a Darwinula Brady y Norman, han sido reasignados recientemente a géneros paleozoicos como Suchonellina Spizharskyi, lo que evidencia la necesidad de una revisión taxonómica del grupo.

*Proyecto financiado por PICT 2014-1964.

\section{POSIBLE DIMORFISMO SEXUAL EN LOS DARWINULOCOPINA DEL TRIÁSICO MEDIO DE MENDOZA}

\author{
A.P. Carignano ${ }^{1}$, J. Echevarría ${ }^{1}$ y A.M. Zavattieri ${ }^{2 *}$
}

${ }^{1}$ Consejo Nacional de Investigaciones Científicas y Tecnológicas (CONICET). División Paleozoología de Invertebrados, Museo de La Plata, Facultad de Ciencias Naturales y Museo, Universidad Nacional de La Plata. Paseo del Bosque s/n, B1900FWA La Plata, Buenos Aires, Argentina. apcarignano@fcnym.unlp.edu.ar; javierechevarria@fcnym.unlp.edu.ar

2Instituto Argentino de Nivología, Glaciología y Ciencias Ambientales (IANIGLA), Centro Científico Tecnológico Mendoza-Consejo Nacional de Investigaciones Científicas y Tecnológicas (CONICET). Avenida Ruiz Leal s/n, 5500 Mendoza, Mendoza, Argentina.amz@mendoza-conicet.gob.ar

Los darwinuloideos (Darwinulocopina, Ostracoda) son considerados como "ancient assexuals", ya que al menos desde el Triásico Medio su reproducción es exclusivamente partenogenética. Esto les confiere un gran interés desde el punto de vista evolutivo. Durante el Paleozoico tardío, en cambio, la gran diversidad que alcanzan los Darwinulocopina se relaciona frecuentemente con la presencia de ambos sexos. Generalmente, en los ostrácodos con dimorfismo sexual, las hembras adultas se reconocen por un mayor ancho en la región posterior del caparazón, que funciona como cámara incubatriz. Morfologías de este tipo se observaron en algunos especímenes del perfil tipo de la Formación Cerro de Las Cabras (Triásico Medio bajo), área de Potrerillos, Precordillera de Mendoza. Para evaluar la significación de estas diferencias se realizó un análisis morfométrico sobre el largo y ancho de los caparazones mejor preservados. Utilizando la regla de Brooks sobre la longitud de los caparazones, se reconocieron al menos tres estadios juveniles y el adulto. Las formas interpretadas como adultos (mayores a $620 \mu \mathrm{m}$ de largo) mostraron una distribución trimodal del ancho con dos límites sin datos: el primero entre los valores 288 y $299 \mu \mathrm{m}$; y el segundo con valores entre 312 y $326 \mu \mathrm{m}$. Se interpreta como más probable que los individuos de menor ancho (36\% de la muestra) sean juveniles A-1 de gran tamaño, los de ancho medio (28\% de la muestra) sean machos y aquellos con anchos mayores ( $36 \%$ de la muestra) sean hembras. Este estudio se complementará con un análisis de morfometría geométrica.

*Proyecto financiado por PICT 2014-1964. 


\title{
ESTUDIO TAFONÓMICO PRELIMINAR DE KATEPENSAURUS GOICOECHEAI DE LA FORMACIÓN BAJO BARREAL (CRETÁCICO SUPERIOR), CUENCA DEL GOLFO SAN JORGE
}

\author{
G.A. Casal ${ }^{1}$, L.M. IbIRICu², B.N. Alvarez ${ }^{3}$, R.D. Martínez ${ }^{1}$ y M. Luna ${ }^{1 *}$ \\ ${ }^{1}$ Laboratorio de Paleontología de Vertebrados, Facultad de Ciencias Naturales y Ciencias de la Salud, Universidad Nacional de la Patago- \\ nia San Juan Bosco. Ruta Provincial n¹, km 4, 9005 Comodoro Rivadavia, Chubut, Argentina.paleogac@yahoo.com.ar; rdfmartinez@yahoo.com; \\ paleoambiental@yahoo.com.ar \\ 2Instituto Patagónico de Geología y Paleontología (IPGP), Centro Nacional Patagónico (CENPAT)-Consejo Nacional de Investigaciones Cien- \\ tíficas y Tecnológicas (CONICET). Boulevard Brown 2915, U9120ACD Puerto Madryn, Chubut, Argentina. ibiricu@cenpat-conicet.gob.ar \\ ${ }^{3}$ Centro de Investigaciones y Transferencia Golfo San Jorge-Consejo Naiconal de Investigaciones Científicas y Tecnológicas (CONICET), Universidad \\ Nacional de la Patagonia San Juan Bosco. Ruta Provincial n 1, km 4, 9005 Comodoro Rivadavia, Chubut, Argentina.bruno.n.alvarez@outlook.com
}

Se analizan los atributos tafonómicos del saurópodo rebaquisáurido Katepensaurus goicoecheai (UNPSJB-PV 1007), procedente de la Formación Bajo Barreal (Cretácico Superior) en el centro-sur de Chubut. Los restos se preservaron en un depósito de desbordamiento no canalizado, asociado a canales fluviales de baja sinuosidad y diseño entrelazado. El depósito presenta geometría lobulada, 20 a $30 \mathrm{~cm}$ de potencia y está integrado por areniscas gruesas con abundante matriz pelítica y tobácea, que permiten caracterizarlo como un flujo hiperconcentrado. Los elementos recuperados corresponden principalmente al esqueleto axial, estaban desarticulados y exhiben fracturas longitudinales y exfoliación en escamas, evidenciando una exposición subaérea prolongada. Presentan abrasión por transporte hidráulico y una reorientación NW-SE, normal a la dirección del paleoflujo. La dispersión lateral es agrupada producto de entrampamientos hidráulicos causados por los restos de mayor tamaño. La escasa potencia del desbordamiento no sepultó completamente los restos, quedando estructuras expuestas que fueron intensamente erosionadas. Posteriormente un nuevo desbordamiento los cubrió completamente. En cuanto a los procesos fosildiagenéticos, los restos exhiben escasa deformación plástica por presión litostática. La estructura ósea, formada originalmente por hidroxiapatita fue modificada a fluorapatita. Los procesos de permineralización en los canales vasculares precipitaron como una primera capa con hematita sobre los bordes de los mismos, luego calcedonia, cuarzo y finalmente zeolitas rellenándolas completamente. La similitud en la composición química de los fósiles y de la roca portadora de los mismos sugiere que el enriquecimiento con nuevos elementos fue debido al intercambio directo con los sedimentos en los que fueron enterrados.

*Proyecto parcialmente financiado por PICT 2016-0459 (a LMI y GAC).

\section{MIDDLE JURASSIC CALCAREOUS NANNOFOSSILS FROM A NEW LOCALITY IN THE LOS MOLLES FORMATION, NEUQUÉN BASIN}

\author{
M. Chaumeil Rodríguez ${ }^{1}$, J.P. Pérez Panera¹, E. Mattioli², and G.N. Angelozzi ${ }^{3 *}$
}

${ }^{1}$ Laboratorio de Bioestratigrafía, YPF Tecnología S.A.-Consejo Nacional de Investigaciones Científicas y Tecnológicas (CONICET). Avenida del Petróleo Argentino s/n entre 129 y 143, 1923 Berisso, Buenos Aires, Argentina.micaela.chaumeil@ypftecnologia.com; juan.p.panera@ypftecnologia.com 2Université Claude Bernard Lyon 1, Laboratoire de Géologie de Lyon: Terre, Planètes, Environnement. Rue Raphaël Dubois, 69622 CEDEX, Villeurbanne, Lyon, Francia. mattioli@univ-lyon1.fr

${ }^{3}$ GEMA S.R.L.-Laboratorio de Bioestratigrafía, YPF Tecnología S.A.. Avenida del Petróleo Argentino s/n entre 129 y 143, 1923 Berisso, Buenos Aires, Argentina. gladys.angelozzi@set.ypf.com

Most of Middle Jurassic nannofossil studies are based upon Tethyan sections and only sporadically coccolith record has been documented from the Neuquén basin (Pacific realm). We present the first detailed report on calcareous nannofossils from this region. The Matuasto III section (Los Molles Formation) crops out near the left margin of arroyo Picún Leufú, 45 m south from the city of Zapala, Neuquén province. It corresponds to $23 \mathrm{~m}$ of massive mudstones with few and thin intercalated fine sandstones; bivalves, ammonites, coal and leaf fragments were recovered. Calcareous nannofossil assemblages had good to moderate preservation and some samples presented high diversity and abundance. Preliminary results indicate that this section records the Aalenian/Bajocian boundary and yields one of the best calcareous nannofossil assemblages for this time-interval for the basin. The base of the section is characterized by Similiscutum novum, Biscutum intermedium, Bussonius prinsii, Discorhabdus striatus, D. criotus, Lotharingius sigillatus and Schizosphaerella punctulata. The presence of Podorhabdus grassei and Watznaueria contracta indicates an age not older than middle Aalenian (NJ8b biozone). Assemblages of the middle and upper part of the section are characterized by Biscutum depravatus, Similiscutum novum, Lotharingius hauffii and L. sigillatus. First occurrence of Watznaueria britannica and the presence of Carinolithus superbus constraints these levels to the early Bajocian (NJ9 biozone). The preliminary data presented here show the high potential of calcareous nannofossil for biostratigraphic reconstructions in the Neuquén basin. Furthermore, relative abundances of some species strongly vary across the section and might be related to environmental factors.

*Proyecto financiado por Y-Tec I+D+i 631, PICT-2014-1271. 


\title{
MEDIDAS DE CONSERVACIÓN EN LAS COLECCIONES DE PALEONTOLOGÍA DE VERTEBRADOS DEL MUSEO ARGENTINO DE CIENCIAS NATURALES “BERNARDINO RIVADAVIA" (MACN)
}

\author{
L. Chornogubsky ${ }^{1}$, M.D. Ezcurra ${ }^{1}$, M. Miñana ${ }^{1}$, G.O. Aguirrezabala ${ }^{1}$ y S. Tancoff $^{1}$ \\ ${ }^{1}$ Museo Argentino de Ciencias Naturales "Bernardino Rivadavia" (MACN)-Consejo Nacional de Investigaciones Científicas y Tecnológicas \\ (CONICET). Av. Angel Gallardo 470, C1405DJR Ciudad Autónoma de Buenos Aires, Argentina. Ichorno@macn.gov.ar; martindezcurra@yahoo.com.ar; \\ laberintodehuesos@hotmail.com; guillecomallo@yahoo.com.ar; stancoff@macn.gov.ar
}

La Sección Paleontología de Vertebrados del MACN alberga dos importantes colecciones nacionales: la de Paleovertebrados (MACN-Pv) y la Colección Nacional Ameghino (MACN-A). Ambas colecciones contienen un total aproximado de 50.000 piezas, entre las cuales unas 1.500 corresponden a ejemplares de tipos portanombre. Continuando con el propósito de poner en valor la colección, comenzado en gestiones previas, se ha realizado un análisis general de la misma y se han detectado tres problemas prioritarios: 1) deterioro y extravío de piezas fósiles; 2) deterioro de mobiliario por plagas; y 3) precariedad en la instalación eléctrica. Respecto de los dos primeros puntos, se comenzó a reacondicionar el material fósil a partir de la utilización de cajas plásticas de alta resistencia mecánica y moderada resistencia a altas temperaturas y camas de espuma de polietileno, con el respectivo censo del material acondicionado. Esto ha permitido la localización de varios materiales que se hallaban extraviados de ambas colecciones. Se proyecta el reemplazo de las estanterías de madera por metálicas y continuar con el acondicionamiento en cajas, mejorando la identificación del material tomando fotografías y utilizando códigos QR. Por otro lado, se realizarán mejoras en las instalaciones eléctricas mediante el recableado de la red, instalación de llaves térmicas y disyuntores.

\section{PRESENCIA DE UN PEDIOMYIDAE EN EL EOCENO DE SALTA}

\author{
L. Chornogubsky ${ }^{1}$, A.N. Zimicz ${ }^{2}$ y F.J. GoIn ${ }^{3 *}$
}

'Museo Argentino de Ciencias Naturales "Bernardino Rivadavia" (MACN)-Consejo Nacional de Investigaciones Científicas y Tecnológicas (CONICET). Av. Angel Gallardo 470, C1405DJR Ciudad Autónoma de Buenos Aires, Argentina. Ichorno@macn.gov.ar

${ }^{2}$ Instituto de Bio y Geociencias del NOA (IBIGEO), Consejo Nacional de Investigaciones Científicas y Técnicas (CONICET)-Universidad Nacional de Salta. 9 de julio 14, A4405BBB Rosario de Lerma, Salta, Argentina. natalia.zimicz@gmail.com

${ }^{3}$ División Paleontología Vertebrados, Museo de La Plata, Facultad de Ciencias Naturales y Museo, Universidad Nacional de La Plata. Paseo del Bosque s/n, B1900FWA La Plata, Buenos Aires, Argentina.fgoin@fcnym.unlp.edu.ar

Recientemente se ha dado a conocer una fauna de metaterios (mamíferos marsupiales y aliados) exhumada en niveles Eocénicos (ca. 40 Ma) de la Formación Quebrada de Los Colorados (FQLC) aflorante en el Parque Nacional Los Cardones (provincia de Salta, Argentina). Dicha fauna incluye una especie de Sparassodonte, dos de Prepidolopidae y un Argyrolagoidea indet. En una nueva campaña a la región fueron hallados dos maxilares con los M2-3 pertenecientes a un nuevo metaterio para la FQLC (incluidos en la colección IBIGEO-P). Se realizó un análisis filogenético incluyendo 50 taxones de metaterios fósiles y actuales y 361 caracteres esqueletarios. El consenso (8 árboles de 1494 pasos) ubica al nuevo taxón (IBIGEO-P) entre los Pediomyidae: (((IBIEGO-P Protolambda hatcheri) Bobbschaefferia sp.) Glasbius). P. hatcheri y Glasbius provienen del Cretácico Tardío de América del Norte mientras que Bobbschaefferia sp., del Eoceno de Sao José de Itaboraí (Brasil). Entre las sinapomorfías que unen a IBIGEO-P con $P$. hatcheri se encuentra la presencia de una plataforma estilar reducida, mientras que en los nodos que incluyen a Bobbschaefferia sp. y Glasbius respectivamente, son la presencia de superficies labiales redondeadas en el paracono y el metacono, la presencia de un metacónulo más alto que la cuenca del trígono y "alado" y una centrocrista recta, entre otras. Este nuevo hallazgo corrobora la similitud entre especies del Noroeste Argentino y aquellas del Eoceno de Brasil, plantea similitudes con aquellas del Cretácico de América del Norte y aumenta la diversidad conocida para el Eoceno del Noroeste Argentino.

*Proyecto financiado por PICT 2014-2497; PICT 201-0508; PUE 22920160100098. 


\title{
LOS DASYPODIDAE (MAMMALIA, XENARTHRA) DEL EOCENO MEDIO (EDAD MAMÍFERO CASAMAYORENSE) DE PATAGONIA
}

\author{
M.R. Ciancio ${ }^{1}$, J.S. Salgado-Ahumada ${ }^{1}$ y A.A. Carlini ${ }^{1 *}$ \\ ${ }^{1}$ Laboratorio de Morfología Evolutiva y Desarrollo (MORPHOS) y División Paleontología de Vertebrados, Museo de La Plata, Facultad de Cien- \\ cias Naturales y Museo, Universidad Nacional de La Plata. Paseo del Bosque s/n, B1900FWA La Plata, Buenos Aires, Argentina. \\ mciancio@fcnym.unlp.edu.ar; flacosalgado@gmail.com; acarlini@fcnym.unlp.edu.ar
}

La fauna de la Edad-Mamífero Casamayorense sensu lato fue equívocamente estructurada a partir de restos colectados por Carlos Ameghino en distintas localidades del centro-este de la provincia del Chubut aunque, sin embargo, la localidad tipo está en Punta Casamayor (Golfo San Jorge, Santa Cruz). Los numerosos Dasypodidae referidos originalmente por Florentino Ameghino al Casamayorense (fines del s. IXX y principios del s. XX), son: tres especies de Astegotheriini (Dasypodinae) y, cuatro de "Utaetini", diez de Euphractini y un Eutatini (Euphractinae). A mediados del s. XX, esa diversidad nominal fue drásticamente reducida por Simpson, ya que sinonimizó varios taxones para este lapso. Posteriormente, se definieron dos subedades con faunas casamayorenses diferentes, "Vaquense" ( 45 Ma, Luteciano, Cañadón Vaca, Chubut) y Barranquense ( $40 \mathrm{Ma}$, Bartoniano, Gran Barranca, Chubut), ambas provenientes de niveles inferiores de la Formación Sarmiento, abarcando un lapso temporal de al menos $4 \mathrm{Ma}$. Luego de extensos trabajos en las localidades tipo, se ha revisado y actualizado críticamente la diversidad de dasipódidos propios de cada una. Como resultado se observa que los Dasypodidae del Eoceno Medio muestran las primeras radiaciones significativas de clados basales, coincidentes con cambios climáticoambientales, y propias de cada subedad. Sin embargo, aún numerosas especies nominales no han sido registradas nuevamente y varias localidades no han podido ser asignadas a ninguna subedad reconocida, quedando referidas al Casamayorense s.l. Considerando que todas las localidades son geográficamente próximas, estas diversidades diferenciales podrían sugerir que en este lapso de más de 4 Ma los Dasypodidae sufrieron otros eventos cladogenéticos, no claramente identificados aún.

*Proyecto financiado por PICT 0-105, PICT -3831, N-871.

\section{LOS GLIPTODONTES DE LA FORMACIÓN CERRO AZUL (MIOCENO TARDÍO, LA PAMPA, ARGENTINA)}

\author{
L.E. CRuZ1 , J.C. Fernicola ${ }^{1}$, R. Bonini² y C.I. Montalvo ${ }^{3 *}$
}

${ }^{1}$ Museo Argentino de Ciencias Naturales "Bernardino Rivadavia" (MACN)-Consejo Nacional de Investigaciones Científicas y Tecnológicas (CONICET). Av. Ángel Gallardo 470, C1405DJR Ciudad Autónoma de Buenos Aires, Argentina. cruzlaurae@gmail.com; jctano@yahoo.com 2Instituto de Investigaciones Arqueológicas y Paleontológicas del Cuaternario Pampeano (INCUAPA)-Departamento de Arqueología, Universidad Nacional del Centro. Av. del Valle 5737, B7400JWI Olavarría, Buenos Aires, Argentina. rbonini@fcnym.unlp.edu.ar

${ }^{3}$ Facultad de Ciencias Exactas y Naturales, Universidad Nacional de La Pampa, Uruguay 151, L6300CLB Santa Rosa, La Pampa, Argentina. cmontalvolp@yahoo.com.ar

Se da a conocer el estudio preliminar de una colección de gliptodontes (Xenarthra, Cingulata) que proceden de nueve asociaciones faunísticas recuperadas de afloramientos de la Formación Cerro Azul. Esta formación está constituida por limos arenosos continentales de composición volcaniclástica que corresponden tanto a facies eólicas (loess; Bajo Giuliani, Loventué, Cerro El Chancho, Conelho, Quehué, Telén) como a fluviales (limos arenosos retrabajados; Laguna Chillhué, Guatraché y Salinas Grandes de Hidalgo). Aflora en el centro y este de la provincia de La Pampa y en el suroeste de la de Buenos Aires; sus faunas corresponden principalmente a la edad Huayqueriense (Mioceno Tardío). Los materiales corresponden a fragmentos de corazas, casquetes cefálicos y tubos caudales. Se reconocieron cuatro géneros Coscinocercus, Hoplophractus, Aspidocalyptus y Eosclerocalyptus. Los tres primeros habían sido mencionados en listados faunísticos de alguna de las localidades portadoras, pero este es el primer estudio que incluye el análisis anatómico detallado de los ejemplares disponibles en cada localidad. La presencia de los tres primeros taxones avala la correlación bioestratigráfica de estos afloramientos de La Pampa con los registrados en Adolfo Alsina (oeste de la provincia de Buenos Aires), y descriptos inicialmente por Cabrera. Asimismo, cabe destacar también que la presencia de Hoplophractus y Eosclerocalyptus permite correlacionar estos materiales con algunos descriptos para la provincia de Catamarca. Finalmente, Coscinocercus y Aspidocalyptus, hasta ahora solo se conocen en esta región central de Argentina por lo que los abundantes materiales recuperados permitirán reconocer con detalle las especies de los géneros identificados, ajustando sus asignaciones taxonómicas.

*Proyecto financiado por PICT 2016-2665. 


\title{
ENDOCRANIAL ANATOMY OF A PALEOCENE STEM WATERFOWL (AVES, ANSERIFORMES)
}

\author{
F.J. Degrange ${ }^{1}$, C.P. Tambussi ${ }^{1}$, L.M. Witmer ${ }^{2}$, M.M. Demmel Ferreira ${ }^{1}$, And S. Santillana ${ }^{3 *}$
}

${ }^{1}$ Centro de investigaciones en Ciencias de la Tierra (CICTERRA)-Consejo Nacional de Investigaciones Científicas y Técnicas (CONICET) and Universidad Nacional de Córdoba. Av. Vélez Sársfield 1611, X5016CGA Córdoba, Córdoba, Argentina. fjdino@gmail.com; tambussi@conicet.gov.ar; manudemmel@gmail.com

2Department of Biomedical Sciences, Ohio University Heritage College of Osteopathic Medicine, Ohio Center for Ecology and Evolutionary Studies. 45701 Athens, Ohio, United States of America. witmerl@ohio.edu

IInstituto Antártico Argentino. 25 de Mayo 1143, B1650HMK San Martín, Buenos Aires, Argentina. ssantillana@dna.gov.ar

Morphology of the avian brain is of critical importance for studies on the evolution of modern birds, and especially important is the information provided by stem avian fossils. Here we present a reconstruction of the endocranial morphology based on computed tomography of a stem representative of Anseriformes of early Paleocene age (Danian). The fossil (MLP 07-III-1-1) comes from the López de Bertodano Formation (Maastrichtian-Danian) of Seymour Island, West Antarctica. The brain cast shows a wide telencephalon, small optic lobes, large olfactory bulbs, a short and wide cerebellum, and a wide medulla oblongata. Viewed laterally, it displays a slight flexure of the main brain axis, and the telencephalon completely covers the mesencephalon in dorsal view. No telencephalic Wulst is present, suggesting that this innovative avian neurological peculiarity was not recognizably developed 65.5 Myr ago. Morphology was closer to that of the Eocene anseriform Presbyornis due to the presence of large olfactory bulbs, stout flocculi that are caudolaterally directed, and also the absence of the Wulst. Absence of the Wulst and small optic lobes seems to indicate that stem Anseriformes were not strongly visually oriented birds whereas its large olfactory bulbs would denote significant olfactory capabilities. In crown Anseriformes (as in most modern birds), the condition is the opposite. Consequently, the brain morphology of the new Antarctic fossil is consistent with morphological trends seen in living birds: replacement of olfactory sensory input by enhanced vision.

*Financial support by PIP-CONICET 0059, IAA, INST 04 of the IAA, Foncyt, PUE 2016 - CONICET-CICTERRA.

\section{NEW REMAINS OF DRYORNIS PAMPEANUS, A FOSSIL CATHARTID FROM THE PLIOCENE OF BUENOS AIRES, ARGENTINA}

\author{
F.J. Degrange ${ }^{1}$, M.L. Taglioretti², G.R.R. Brito ${ }^{3}$, F. Scaglia² y C.P. Tambussi ${ }^{1 *}$
}

${ }^{1}$ Centro de investigaciones en Ciencias de la Tierra (CICTERRA)-Consejo Nacional de Investigaciones Científicas y Técnicas (CONICET) and Universidad Nacional de Córdoba. Av. Vélez Sársfield 1611,X5016CGA Córdoba, Córdoba, Argentina. fjdino@gmail.com; tambussi@conicet.gov.ar ${ }^{2}$ Museo Municipal de Ciencias Naturales Lorenzo Scaglia. Plaza España sin número, 7600 Mar del Plata, Buenos Aires, Argentina. paleomat@yahoo.com.ar; feroscaglia@gmail.com

${ }^{3}$ Departamento de Ecologia e Zoologia-Centro de Ciências Biológicas (ECZ/CCB), Universidade Federal de Santa Catarina. Campus Universitário s/n - sala 218B, 88040-900 Florianópolis, Santa Catarina, Brasil. g.brito@ufsc.br

Cathartidae ("new world vultures"), characterized by their soaring flight and carrion based trophic habits, are found in warm and temperate zones of the Americas, where they are recorded from the Eocene. New remains of the putative cathartid Dryornis pampeanus Moreno and Mercerat provide more information about the species, originally named in 1891 based on a distal fragment of right humerus proceeding from the Monte Hermoso Formation (Early Pliocene). The new materials MMP 5731 were unearthed in La Estafeta beach, Mar del Plata, Buenos Aires province, from the upper levels of the Chapadmalal Formation, corresponding to the paleosoil 6 of the Playa Los Lobos Alloformation, dated in 3.3 Ma, upper Pliocene. Materials consist of a fragmentary tibiotarsus and articulated right pectoral girdle and partial forelimb: complete right humerus, proximal ends of the right ulna and radius, right coracoid, right incomplete scapula, and fragment of sternum. This new finding extends the biocron of Dryornys pampeanus to the upper Chapadmalalan age, shortening the faunal differences with the Montehermosan age. Dryornis body mass estimation is $26161 \mathrm{gr}$ (based on the length of the humeral articulation facet of the coracoid: $28.48 \mathrm{~mm}$ ), being the largest cathartid registered until now. Preliminary phylogenetic analyses show that Dryornis may be a basal giant common vulture, sister to the (Cathartes + Coragyps) clade, although its position among Cathartiformes still seems controversial. The new specimen provides detailed new morphological data for Dryornis pampeanus, which will be important for future evaluations of the phylogenetic relationships and evolution of the Cathartiformes clade.

*Financial support by PICT 2330. 


\section{NUEVOS HALLAZGOS Y DATACIONES EN LA FORMACIÓN ISCHIGUALASTO EN CERRO LAS LAJAS (CUENCA ISCHIGUALASTO-VILLA UNIÓN), PROVINCIA DE LA RIOJA, ARGENTINA}

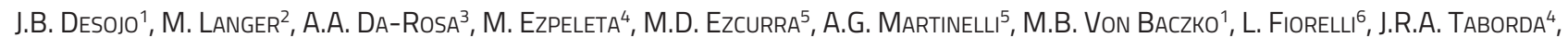
M.J. Trotteyn 7 , M. HeChenleitner ${ }^{6}$, F. Montefeltro ${ }^{8}$ Y J. Ramezan $I^{9 *}$

1'División Paleontología Vertebrados, Museo de La Plata, Facultad de Ciencias Naturales y Museo, Universidad Nacional de La Plata. Paseo del Bosque s/n, B1900FWA La Plata, Buenos Aires, Argentina.julideso2@gmail.com; belenvonbaczko@gmail.com

2Departamento de Biologia, Faculdade de Filosofia, Ciencias e Letras, Universidade de Sao Paulo. Av. Bandeirantes 3900, 14040-901 Ribeirao Preto, São Pablo, Brasil. langer.mc@gmail.com

${ }^{3}$ Universidade Federal de Santa Maria, Centro de Ciências Naturais e Exatas, Departamento de Geociências, Laboratório de Estratigrafia e Paleobiologia. Camobi, 97.105-900 Santa Maria, Rio Grande do Sul, Brasil. atiladarosa@gmail.com

${ }^{4}$ Centro de investigaciones en Ciencias de la Tierra (CICTERRA)-Consejo Nacional de Investigaciones Científicas y Técnicas (CONICET) and Universidad Nacional de Córdoba. Av. Vélez Sársfield 1611, X5016CGA Córdoba, Córdoba, Argentina.

miguelezpeleta@gmail.com; jeremias.taborda@gmail.com

${ }^{5}$ Sección Paleontología de Vertebrados, Museo Argentino de Ciencias Naturales "Bernardino Rivadavia" (MACN)-Consejo Nacional de Investigaciones Científicas y Tecnológicas (CONICET). Av. Ángel Gallardo 470, C1405DJR Ciudad Autónoma de Buenos Aires, Argentina.

martindezcurra@yahoo.com.ar; aeolosagm@gmail.com

${ }^{6}$ Centro Regional de Investigaciones Científicas y Transferencia Tecnológica de La Rioja (CRILAR)-Consejo Nacional de Investigaciones Científicas y Tecnológicas (CONICET). Entre Ríos y Mendoza s/n, 5301 Anillaco, La Rioja, Argentina. Iucasfiorelli@gmail.com; emhechenleitner@gmail.com

${ }^{7}$ Centro de Investigaciones de la Geósfera y la Biósfera (CIGEOBIO), Consejo Nacional de Investigaciones Científicas y Tecnológicas (CONICET). Instituto de Geología "Emiliano Pedro Aparicio", Departamento de Geología, Facultad de Ciencias Exactas, Físicas y Naturales, Universidad Nacional de San Juan. Av. de la Roza Oeste 590, J5402DCS San Juan, San Juan, Argentina. jtrotteyn@gmail.com

${ }^{8}$ Departamento de Biologia e Zootecnia, Universidade Estadual Paulista, FEIS. Passeio Monção 226, 15385000 Ilha Solteira, Sao Paulo, Brasil. felipecmontefeltro@gmail.com

9Massachusetts Institute of Technology. Av. 77, 02139 Cambridge, Massachusetts, United States of America. ramezani@mit.edu

El contenido de tetrápodos fósiles de la Formación Ischigualasto representa uno de los mejores registros de asociaciones faunísticas continentales del Carniano superior-Noriano inferior a nivel mundial. La gran mayoría de sus fósiles fueron colectados en la Hoyada de Ischigualasto (HI) (San Juan) y en menor medida en la localidad menos explorada de Cerro Las Lajas (CLL) (La Rioja). El registro fosilífero édito de esta última localidad se encontraba restringido a los holotipos del ornitosúquido Venaticosuchus rusconii y del dinosauriforme Pisanosaurus mertii, y un ejemplar referido al crocodylomorfo Trialestes romeri. Actualmente no existe consenso sobre la correlación estratigráfica entre los niveles aflorantes en el CLL y aquellos de la HI. En los últimos 5 años hemos realizado tres campañas paleontológicas en las rocas de la Formación Ischigualasto aflorantes en CLL con el objetivo de expandir el registro de vertebrados de esta unidad en La Rioja. Damos a conocer nuevos materiales del tercio inferior (70-200 m) de la Formación Ischigualasto en el CLL que comprenden los primeros registros para la localidad de rincosaurios hyperodapedontinos (incluyendo dos nuevos taxones), del traversodóntido Exaeretodon sp., del proterochámpsido Proterochampsa sp., un aetosaurio y un probable poposauroideo no-shuvosáurido. Exceptuando las dos especies de rincosaurios y el probable poposauroideo, los otros taxones fueron registrados previamente en la mitad inferior de la formación en la HI. Dataciones radioisotópicas (U-Pb) realizadas en distintos niveles estratigráficos permiten proponer por primera vez que la sucesión faunística del tercio inferior de CLL sería equivalente a la biozona de ScaphonyxExaeretodon-Herrerasaurus registrada en la HI.

*Proyecto financiado por PICT 2014-0609 (J.B.D). 


\title{
QUISTES DE DINOFLAGELADOS DEL MIEMBRO SUPERIOR DE LA FORMACIÓN RÍO TURBIO (EOCENO MEDIO A SUPERIOR): NUEVOS APORTES A LA EDAD
}

\author{
A.V. Doldan ${ }^{1}$, C.R. Amenábar ${ }^{1}$, B. Córdoba² y G.R. Guerstein ${ }^{3 *}$ \\ ${ }^{1}$ Instituto de Estudios Andinos "Don Pablo Groeber" (IDEAN), Universidad de Buenos Aires-Consejo Nacional de Investigaciones Científi- \\ cas y Tecnológicas (CONICET). Intendente Güiraldes 2160, Ciudad Universitaria - Pabellón II, C1428EGA Ciudad Autónoma de Buenos Aires, \\ Argentina.anidoldan@gmail.com; amenabar@gl.fcen.uba.ar \\ 2Departamento de Ciencias Geológicas, Facultad de Ciencias Exactas y Naturales, Universidad de Buenos Aires-Consejo Nacional de Investi- \\ gaciones Científicas y Tecnológicas (CONICET). Intendente Güiraldes 2160, Ciudad Universitaria - Pabellón II, C1428EGA Ciudad Autónoma de \\ Buenos Aires, Argentina. bea_cordoba80@hotmail.com \\ ${ }^{3}$ Instituto Geológico del Sur (INGEOSUR), Consejo Nacional de Investigaciones Científicas y Tecnológicas (CONICET)-Departamento de Geolo- \\ gía, Universidad Nacional del Sur (UNS). Av. Alem 1253, B8000CPB Bahía Blanca, Buenos Aires, Argentina. raquel.guerstein@uns.edu.ar
}

En este trabajo se presentan las asociaciones de quistes de dinoflagelados del miembro superior de la Formación Río Turbio, Cuenca Austral. Se trata de nuevos resultados de un muestreo de mayor resolución sobre perfiles de afloramientos en la Ruta Nacional 40 y testigos corona del Pozo CA-1, perforado por YCF (5130' S y $72^{\circ} 18^{\prime}$ O), cercanos a la localidad de Río Turbio. Teniendo en cuenta la biozonación de dinoflagelados propuesta para la Cuenca Austral y la presencia de las especies endémicas de altas latitudes del Hemisferio Sur, entre ellas Arachnodinium antarcticum y altas frecuencias de Enneadocysta dictyostila en las muestras inferiores del afloramiento, es posible ubicar a este intervalo en la biozona RTF2, de edad mayor a $39 \mathrm{Ma}$ (Bartoniano tardío). En las muestras superiores E. dictyostila disminuye y aumentan los registros del complejo de Turbiosphaera, sugiriendo que este intervalo representa la transición hacia la Zona RTF3 (Bartoniano tardío-Priaboniano medio, 39-36 Ma). Las muestras de la parte inferior del testigo contienen especies endémicas cuyas últimas ocurrencias abundantes se extienden hasta el Priaboniano temprano (Vozzhennikovia spp., Spinidinium macmurdoense, complejo de Turbiosphaera). En los niveles superiores del testigo el primer registro de Alterbidinium distinctum indica una edad no mayor a los 35.95 Ma, comparable con la Zona RTF3. Por debajo del contacto entre las Formaciones Río Turbio y Río GuiIlermo se observa el reemplazo de especies endémicas por especies cosmopolitas, lo que caracteriza a la Zona RTF4. El techo de dicha zona se registra dentro del Magnetocrón C13r, en la transición Eoceno/Oligoceno.

*Proyetco financiado por PGI 24/F066.

\section{PRIMER REGISTRO PALEOFLORÍSTICO PARA LA FORMACIÓN MONINA, TRIÁSICO MEDIO, DEPOCENTRO HILARIO, CUENCA CUYANA, SAN JUAN, ARGENTINA}

\author{
J.M. Drovandi, ${ }^{1,2}$, G.A. Correa ${ }^{1}$, E.P. Coturel ${ }^{3}$, C.E. Colombi ${ }^{1,2}$, J. Bodnar ${ }^{3}$ y E.M. Morel ${ }^{3 *}$ \\ ${ }^{1}$ Instituto y Museo de Ciencias Naturales (IMCN), Universidad Nacional de San Juan. España 400 (norte), J5400DNQ San Juan, San Juan, \\ Argentina.gustavoalejandrocorrea@yahoo.com.ar; ccolombi@unsj.edu.ar \\ ${ }^{2}$ Centro de Investigaciones de la Geósfera y la Biósfera (CIGEOBIO), Consejo Nacional de Investigaciones Científicas y Tecnológicas (CONICET)- \\ Facultad de Ciencias Exactas, Físicas y Naturales, Universidad Nacional de San Juan, Argentina. jdrovandi@unsj.edu.ar \\ ${ }^{3}$ División Paleobotánica, Museo de La Plata, Facultad de Ciencias Naturales y Museo, Universidad Nacional de La Plata. Paseo del Bosque s/n, \\ B1900FWA La Plata, Buenos Aires, Argentina.ecoturel@fcnym.unlp.edu.ar; jbodnar@fcnym.unlp.edu.ar; emorel@museo.fcnym.unlp.edu.ar
}

Los afloramientos de la Cuenca Cuyana ubicados en la Precordillera Occidental sanjuanina se distribuyen en tres depocentros principales: Rincón Blanco, Barreal e Hilario. En el depocentro Hilario se reconocieron cuatro formaciones, que de base a techo son: Agua de los Pajaritos, Monina, Hilario y El Alcázar. En esta contribución se da a conocer el primer registro de restos paleoflorísticos para la Formación Monina. Esta unidad presenta un espesor de 150 m y se caracteriza por depósitos de pelitas y subordinados bancos areniscosos finos a medios y tobas, interpretado como un paleoambiente deltaico/lacustre somero. En esta formación se identificaron dos estratos fosilíferos (EF). El primero (EF1) ubicado a 15 m de la base, preserva impresiones-compresiones de material carpológico y fragmentos de hojas, el segundo (EF2) se ubica a $22 \mathrm{~m}$ de la base y preserva restos de troncos permineralizados. En el EF1 se identificaron: esporofilos de licofitas aislados correspondientes al género Lepacyclotes sp. (Emmons) Retallack (Isoetales); un fragmento de hoja atribuido a Delnortea cf. abottiae Mamay, Miller, Rohr y Stein (Peltaspermales); dos estructuras reproductivas, una asignada al género Peltaspermum Harris emend. Townrow (Peltaspermales), y la segunda al género Telemachus Anderson (Voltziales); semillas atribuibles al género Cordaicarpus sp. Geinitz y a Andersonia cf. rastroensis Arce y Lutz. En el EF2 se colectaron fragmentos de leños permineralizados que observados en los tres planos de corte, muestran afinidad con el género Protocircoporoxylon Vogellehner (Coniferales). La presencia de estos restos, complementan el contexto paleoflorístico ya conocido para la Formación El Alcázar en el depocentro Hilario. 


\title{
EVOLUCIÓN DEL ORDEN TRIGONIIDA (MOLLUSCA-BIVALVIA) DURANTE LA TRANSICIÓN TRIÁSICO/JURÁSICO Y SUS IMPLICACIONES EN LA SISTEMÁTICA DEL GRUPO
}

\author{
J. Echevarría ${ }^{1}$ y S. Ros Franch ${ }^{1}$
}

${ }^{1}$ Consejo Nacional de Investigaciones Científicas y Técnicas (CONICET). División Paleozoología Invertebrados, Museo de La Plata, Facultad de Ciencias Naturales y Museo. Paseo del Bosque s/n, B1900FWA La Plata, Buenos Aires, Argentina. javierechevarria@fcnym.unlp.edu.ar; soniaros@fcnym.unlp.edu.ar

Tras una importante radiación durante el Triásico (Ilegando a reconocerse diecisiete géneros en el Noriano), los trigónidos sufrieron una fuerte reducción durante la extinción del final del período (cuatro géneros registrados en el Hettangiano, solo dos proviniendo del Triásico). La radiación posterior modeló el acmé del grupo, por lo que su adecuada comprensión resulta fundamental para definir la sistemática dentro del orden. Mientras que durante el Triásico tuvieron una distribución cosmopolita, la región Pacífica funcionó como refugio para el grupo durante la crisis. Los géneros Trigonia Bruguière (sobreviviente triásico), Geratrigonia Kobayashi y Mori, y Vaugonia Crickmay aparecen en el Hettangiano de Japón, mientras que Prosogyrotrigonia Krumbeck (también del Triásico) reaparece en el Hettangiano de Chile. Las marcadas diferencias morfológicas entre los dos sobrevivientes triásicos sugieren la existencia de dos linajes separados. Geratrigonia comparte varios caracteres con Trigonia y formas triásicas vinculadas, mientras que Vaugonia podría representar una tercera línea cruzando el límite Triásico/Jurásico. Durante el Sinemuriano aparecen los géneros Groeberella H. Leanza, Frenguelliella A. Leanza y Jaworskiella A. Leanza, todos asociados al margen Pacífico del continente americano. Groeberella difiere marcadamente de todos los trigónidos jurásico-cretácicos, pero muestra claras afinidades con especies del Triásico. Frenguelliella podría haber evolucionado durante el Sinemuriano a partir de Prosogyrotrigonia o podría provenir de ancestros triásicos (más próximos a Trigonia). Jaworskiella evolucionó en el Jurásico a partir de Frenguelliella; Vaugonia estaría próxima a este linaje según algunos autores. Solo cuando estas relaciones fundamentales sean comprendidas se podrá postular un esquema sistemático estable para el orden.

\section{REVISIÓN TAXONÓMICA DE PROTYPOTHERIUM AMEGHINO (INTERATHERIIDAE, TYPOTHERIA, NOTOUNGULATA) DEL SANTACRUCENSE (MIOCENO TEMPRANO) DE LA PROVINCIA DE SANTA CRUZ, ARGENTINA}

\author{
M. Fernández ${ }^{1,2}$, J.C. Fernicola ${ }^{1,2}$ y E. Cerdeño ${ }^{3 *}$
}

\begin{abstract}
1Departamento de Ciencias Básicas, Universidad Nacional de Luján. Ruta 5 y Av. Constitución, 5700 Luján, Buenos Aires, Argentina. mechi_985@hotmail.com

2División Paleontología de Vertebrados, Museo Argentino de Ciencias Naturales "Bernardino Rivadavia" (MACN)-Consejo Nacional de Investigaciones Científicas y Tecnológicas (CONICET). Av. Ángel Gallardo 470, C1405DJR Ciudad Autónoma de Buenos Aires, Argentina. jctano@macn.gov.ar

${ }_{3}^{3}$ Paleontología, Instituto Argentino de Nivología, Glaciología y Ciencias Ambientales (IANIGLA), Centro Científico Tecnológico MendozaConsejo Nacional de Investigaciones Científicas y Tecnológicas (CONICET). Avenida Ruiz Leal s/n, 5500 Mendoza, Mendoza, Argentina. espe@mendoza-conicet.gob.ar
\end{abstract}

Los Interatheriinae conforman un peculiar grupo de notoungulados Typotheria, ampliamente representados desde el Oligoceno al Mioceno de Sudamérica. En 1885, Florentino Ameghino fundó el primer género interaterino, Protypotherium, en el cual incluyó 11 especies erigidas a partir de ejemplares recolectados en distintos afloramientos de la Formación Santa Cruz (Mioceno Temprano), provincia homónima argentina, correspondiente a la Edad Mamífero Santacrucense. En 1909, en la primera revisión taxonómica de los Typotheria, William Sinclair validó tres especies de Protypotherium ( $P$. australe, $P$. attenuatum y P. praerutilum) y consideró a dos especies ( $P$. claudum y $P$. diversidens) como Typotheria incertae sedis. Posteriormente, en 1996, Adan Tauber sostuvo las sinonimias planteadas por Sinclair, enmendó sus diagnosis, y sinonimizó a $P$. claudum con $P$. australe, pero omitió la situación nomenclatural de $P$. diversidens; este es el esquema vigente. Un estudio taxonómico de las especies santacrucenses del género, basada, particularmente, en el estudio de los ejemplares tipo y en elementos de otras colecciones, permite proponer, preliminarmente, un nuevo esquema taxonómico en el cual se reconocen cuatro morfotipos que se diferencian en base a las características de su dentición yugal y tallas cráneo-mandibulares. Estos morfotipos están representados por las especies $P$. australe, $P$. praerutilum, $P$. compressidens y $P$. claudum.

*Proyecto financiado por PICT 2013-0389, UNLP N/867 y CBLUJ 0000030-18. 


\title{
REVISIÓN SISTEMÁTICA DE ARARIPESUCHUS BUITRERAENSIS POL Y APESTEGUİA: NUEVOS APORTES A LA DIVERSIDAD DE CROCODILIFORMES DE LA BUITRERA, PATAGONIA, ARGENTINA
}

\author{
M.L. Fernández Dumont ${ }^{1}$, P. Bona² y S. Apesteguía ${ }^{1}$ \\ ${ }^{1}$ Fundación de Historia Natural Félix de Azara, Centro de Estudios Biomédicos, Biotecnológicos, Ambientales y Diagnóstico (CEBBAD)-Uni- \\ versidad Maimónides. Hidalgo 775, C1405CBK Ciudad Autónoma de Buenos Aires, Argentina.mlucilafd@gmail.com; sebapesteguia@gmail.com \\ 2División Paleontología de Vertebrados, Unidades de Investigación Anexo Museo, Facultad de Ciencias Naturales y Museo, Universidad Na- \\ cional de La Plata. Av. 60 y 122 s/n, B1904 La Plata, Buenos Aires, Argentina. paulabona26@gmail.com
}

El género Araripesuchus Price (Crocodyliformes, Mesoeucrocodylia) del Cretácico Superior de Gondwana cuenta con seis especies distribuidas entre Níger, Madagascar, Brasil, y Argentina. Las especies argentinas proceden de la Formación Candeleros (Cenomaniano): A. patagonicus Ortega, Gasparini, Buscalioni y Calvo y A. buitreraensis. El objetivo del presente trabajo es ampliar el conocimiento de $A$. buitreraensis, con nuevos materiales craneanos recolectados en La Buitrera (la misma localidad del holotipo, MPCA-PV 235). A partir del estudio anatómico de estos ejemplares, se pueden identificar dos morfotipos, uno representado por los especímenes MPCA-PV 242, 243, 513 y 515, con una morfología más similar al holotipo, lo que permitiría referirlos a esa especie. Para testear esta hipótesis en contexto filogenético mediante el programa TNT 1.5 , los ejemplares fueron incluidos como terminales de una matriz morfológica publicada de Crocodyliformes. Como resultado, los especímenes se recuperan formando un clado anidado dentro de Uruguaysuchidae junto con MPCA-PV 235, separado del resto de los especímenes estudiados (recuperados junto con A. patagonicus y Uruguaysuchus Rusconi, por fuera de este clado), y soportado por caracteres novedosos para A. butreraensis: superficie dorsal de frontal-parietal con cresta media, ausencia de supraoccipital en el techo craneano, sínfisis dentaria más larga que ancha en el proceso anterior, entre otros. Se concluye entonces que el género Araripesuchus en la localidad de La Buitrera podría estar representado por al menos dos especies, que $A$. buitreraensis es una especie válida y que su diagnosis original debe ser enmendada conforme a estas nuevas evidencias.

\section{DON DÁMASO ANTONIO LARRAÑAGA Y EL MEGATHERIUM ACORAZADO: UNA APROXIMACIÓN A LAS CLASIFICACIONES DE LOS MAMÍFEROS DEL SIGLO XVIII Y XIX}

\author{
J.C. FERNICOLA ${ }^{1,2 *}$ \\ 1'Departamento de Ciencias Básicas, Universidad Nacional de Luján. Ruta 5 y Av. Constitución, 5700 Luján, Buenos Aires, Argentina. \\ 2División Paleontología de Vertebrados, Museo Argentino de Ciencias Naturales "Bernardino Rivadavia" (MACN)-Consejo Nacional de In- \\ vestigaciones Científicas y Tecnológicas (CONICET). Av. Ángel Gallardo 470, C1405DJR Ciudad Autónoma de Buenos Aires, Argentina. \\ jctano@macn.gov.ar
}

En 1823, Cuvier publicó una nota a pie de página que daba cuenta de una misiva enviada a Auguste de Saint-Hilaire por el montevideano Padre Dámaso A. Larrañaga, quien además de su vocación religiosa y notable actuación en la política del s. XIX fue un versátil naturalista. En dicha misiva, Larrañaga ubicó al Megatherium, nominado por Cuvier en 1796, como un subgénero de los armadillos: "Dasypus (Megatherium Cuv.)", rechazando así la asignación a los perezosos del mismo. La repercusión que tuvo la carta de Larrañaga se aprecia en las reconstrucciones de época en las que el megaterio porta una coraza o en las asignaciones a megaterio que Charles Darwin asentó en su diario de viaje a partir de los fragmentos de coraza por él recolectados en América del Sur. En 1839, Richard Owen demostró que los megaterios no tenían coraza y que estas pertenecían a un grupo de mamíferos por él denominado gliptodontes. Para algunos autores la asignación subgenérica de Larrañaga fue producto del presunto hallazgo de una coraza junto con el esqueleto de megaterio que fuera nominado por Cuvier. Por el momento no es posible descartar esta idea. Por otra parte, la revisión de los escritos de Larrañaga permite reconocer que ciertos caracteres cráneo-dentarios presentes en el megaterio lo llevaron a posicionarlo como un armadillo ya que dichos caracteres eran los que definían los distintos órdenes de mamíferos reconocidos en aquel entonces por los naturalistas del Viejo Mundo.

*Proyecto financiado por PICT 2016-2665, UNLu CDD-CB 580-16. 


\title{
UNA APROXIMACIÓN A LA OBRA DE FLORENTINO AMEGHINO MEDIANTE EL ESTUDIO DE SUS COLECCIONES, CATÁLOGOS Y PUBLICACIONES
}

\author{
J.C. FERNICOLA ${ }^{1,2 *}$ \\ 1Departamento de Ciencias Básicas, Universidad Nacional de Luján. Ruta 5 y Av. Constitución, 5700 Luján, Buenos Aires, Argentina. \\ 2División Paleontología de Vertebrados, Museo Argentino de Ciencias Naturales "Bernardino Rivadavia" (MACN)-Consejo Nacional de In- \\ vestigaciones Científicas y Tecnológicas (CONICET). Av. Ángel Gallardo 470, C1405DJR Ciudad Autónoma de Buenos Aires, Argentina. \\ jctano@macn.gov.ar
}

En 1878, Florentino Ameghino se embarcó hacia Francia con el objetivo de participar en la Exposición Universal de París y entrar en contacto con destacados naturalistas europeos. En este viaje llevó parte de su primera colección personal de vertebrados fósiles pampeanos que vendió en 1878 al paleontólogo norteamericano Edward D. Cope. Entre 1881, fecha en que regresó a Buenos Aires, y 1886 conformó su segunda colección principalmente con ejemplares pampeanos, que en 1886 fueron vendidos al Museo de La Plata junto con aquellos que no había llevado o vendido en París. Esta venta formó parte del acuerdo alcanzado entre Ameghino y el director de dicha institución, Francisco P. Moreno, para ser nombrado Secretario Subdirector. En 1888, Florentino fue exonerado del Museo de La Plata, dando inicio a la tercera colección particular formada mayoritariamente por ejemplares, que culminó en 1902 al ser nombrado Director del actual Museo Argentino de Ciencias Naturales "Bernardino Rivadavia". De cada una de estas colecciones particulares Ameghino confeccionó un catálogo. De estos solo se preserva el de la tercera colección, en el que es posible encontrar piezas que corresponden a la primera y segunda colección particular. Hasta cierto punto, este hecho justifica la ausencia de materiales tipo en otras colecciones. Por otra parte, en el catálogo existente se brinda procedencia y año de recolección de los ejemplares, información que en ciertos casos contradice la distribución espacio temporal de las asociaciones faunísticas de Ameghino, poniendo en discusión la confiabilidad de los datos por él publicados.

*Proyecto financiado por PICT 2016-2665, UNLu CDD-CB 580-16.

\section{NEW CYNODONT SPECIMENS FROM LOS COLORADOS FORMATION}

\author{
L.C. Gaetano ${ }^{1}$, P. Alonso Muruaga², V. Krapovickas ${ }^{1}$, J.M. Leardi ${ }^{3}$, A. Otero ${ }^{4}$, and C. Apaldetti 5 \\ ${ }^{1}$ Instituto de Estudios Andinos "Don Pablo Groeber" (IDEAN), Universidad de Buenos Aires-Consejo Nacional de Investigaciones Científi- \\ cas y Tecnológicas (CONICET). Intendente Güiraldes 2160, Ciudad Universitaria - Pabellón II, C1428EGA Ciudad Autónoma de Buenos Aires, \\ Argentina.Icgaetano@gl.fcen.uba.ar; veronicakrapovickas@gmail.com \\ ${ }^{2}$ Instituto de geociencias básicas, aplicadas y ambientales de Buenos Aires (IGEBA), Consejo Nacional de Investigaciones Científicas y Tecno- \\ lógicas (CONICET)-Universidad de Buenos Aires (UBA). Intendente Güiraldes 2160, C1428EGA Ciudad Autónoma de Buenos Aires, Argentina. \\ pablojoaquin3@gmail.com \\ ${ }^{3}$ Departamento de Biodiversidad y Biología Experimental, Facultad de Ciencias Exactas y Naturales Universidad de Buenos Aires. Intendente \\ Güiraldes 2160, C1428EGA Ciudad Autónoma de Buenos Aires, Argentina.jmleardi@gl.fcen.uba.ar \\ ${ }^{4}$ División Paleontología de Vertebrados, Unidades de Investigación Anexo Museo, Facultad de Ciencias Naturales y Museo, Universidad \\ Nacional de La Plata. Av. 60 y 122 s/n, B1904 La Plata, Buenos Aires, Argentina. alexandros.otero@gmail.com \\ 5 Instituto y Museo de Ciencias Naturales (IMCN), Universidad Nacional de San Juan. España 400 (norte), J5400DNQ San Juan, San Juan, \\ Argentina.capaldetti@unsj.edu.ar
}

Renovated exploration efforts since 2014 led to new findings in the Late Triassic Los Colorados Formation at the Parque Nacional Talampaya (La Rioja, Argentina). Herein we present four cynodont specimens found in the uppermost third of the unit, represented by skulls with articulated lower jaws. The fossils are concentrated in a massive- to parallel-laminated sandy mudstone interval locally interbedded with parallel- to rippled- laminated sandstone, representing deposition in a floodplain setting sporadically affected by sandy splays from the fluvial channels. They are incomplete and, although fractured, the bone seems mostly undeformed. The new specimens (housed at the UNLR) range from 30\% smaller to slightly larger than the size to those previously reported Chaliminia musteloides Bonaparte individuals, the only named cynodont from Los Colorados Formation. One of the new specimens shows large upper canines, small upper I1 and I2 and lower i1, lower postcanines with a main central cusp flanked a mesial and a distal accessory cusp, and a coronoid process forming a low angle with the horizontal ramus of the dentary. Other specimen is notable by its low skull, delicate, long and low coronoid process, and the absence of a marked step angle at the ventroposterior region of the dentary. A third specimen has very large upper and lower first incisors with the I1 directed anteriorly, a relatively low horizontal ramus of the dentary, and the absence of a diastema between right and left upper incisors. Our preliminary analysis suggests that these three specimens may represent previously unregistered forms.

*Financial support by PIP 11220150100760CO; PICT -2014-1921; PICT 2015-0504; Explorers Club. 


\title{
NEW INSIGHTS ON THE CRANIAL ANATOMY OF EXAERETODON: INTRASPECIFIC VARIATION AND TAXONOMIC IMPLICATIONS
}

\author{
L.C. Gaetano ${ }^{1}$, R. Martínez ${ }^{2}$ y F. Abdala ${ }^{3 *}$ \\ ${ }^{1}$ Instituto de Estudios Andinos "Don Pablo Groeber" (IDEAN), Universidad de Buenos Aires-Consejo Nacional de Investigaciones Cientí- \\ ficas y Tecnológicas (CONICET). Intendente Güiraldes 2160, Ciudad Universitaria - Pabellón II, C1428EGA Ciudad Autónoma de Buenos Aires, \\ Argentina.Icgaetano@gl.fcen.uba.ar \\ ${ }^{2}$ Instituto y Museo de Ciencias Naturales (IMCN), Universidad Nacional de San Juan. España 400 (norte), J5400DNQ San Juan, San Juan, \\ Argentina.martinez@unsj.edu.ar \\ ${ }^{3}$ Unidad Ejecutora Lillo, Consejo Nacional de Investigaciones Científicas y Tecnológicas (CONICET)-Fundación Miguel Lillo. Miguel Lillo 251, \\ T4000JFE San Miguel de Tucumán, Tucumán, Argentina. viutiabdala2@gmail.com
}

Exaeretodon is a very abundant non-mammalian cynodont from the Upper Triassic found in South America and India. Presently, two species are considered valid: E. argentinus Cabrera from Argentina and E. riograndensis Abdala, Barberena and Dornelles from Brazil. We studied several unpublished specimens tentatively assigned to $E$. argentinus from the Ischigualasto Formation (San Juan province, Argentina) that range from $\sim 130 \mathrm{~mm}$ to $\sim 400 \mathrm{~mm}$ in basal skull length. Regardless of the individual size, two morphotypes were identified on the basis of differences in the zygomatic region, including: the presence/ absence of a process of the jugal; the shape, relative orientation, and posterior extension of the dorsal and ventral zygomatic rami of the jugal; and the morphology and orientation of the zygomatic portion of the squamosal. The holotype of $E$. argentinus (formerly Belesodon argentinus Cabrera) is an incomplete left lower jaw. Only the holotypes of the once valid $E$. frenguelli Cabrera, and Theropsis robusta Cabrera (presently considered junior synonyms of E. argentinus) and E. riograndensis preserve the relevant portions of the zygomatic region. These specimens represent only one of the morphotypes whereas the second morphotype has not been previously recognized. In addition, there are differences among the specimens of the first morphotype that were originally interpreted to be due to preservational artifacts but could instead account for intraspecific variation. Our preliminary results suggest that the second morphotype might characterize a new taxon, highlighting the importance of an integral re-evaluation of Exaeretodon considering all the evidence available.

*Financial support by Asociación Paleontológica Argentina (Beca a Jóvenes Investigadores).

\section{ANÁLISIS COMPARATIVO DE LA MICROESTRUCTURA ÓSEA EN VÉRTEBRAS DE UN PLESIO- SAURIO DE ANTÁRTIDA}

\author{
L. Garat ${ }^{1}$, M. Talevi ${ }^{2}$ y M. Reguero ${ }^{3 *}$ \\ 'Universidad Nacional de Río Negro. Estados Unidos 750, R8332BRJ General Roca, Río Negro, Argentina. Igarat@unrn.edu.ar \\ 2Instituto de Investigación en Paleobiología y Geología (IIPG)-Consejo Nacional de Investigaciones Científicas y Tecnológicas (CONICET), Uni- \\ versidad Nacional de Río Negro. Av. Roca 1242, R8332EXZ Gral. Roca, Río Negro, Argentina. mtalevi@unrn.edu.ar \\ ${ }^{3}$ Instituto Antártico Argentino. División Paleontología Vertebrados, Museo de La Plata, Facultad de Ciencias Naturales y Museo, Universidad \\ Nacional de La Plata. Paseo del Bosque s/n, B1900FWA La Plata, Buenos Aires, Argentina. regui@fcnym.unlp.edu.ar
}

Los miembros del orden Plesiosauria son un clado de Sauropterygia adaptados a la vida acuática, cuyo biocrón se extiende desde el Triásico Tardío hasta el Cretácico Tardío. Su distribución es cosmopolita, habiéndoselos registrado en todos los continentes incluyendo la Antártida. Con el objetivo de analizar la variación inter e intravertebral, se estudió comparativamente la microestructura ósea de cuatro elementos que representan distintas porciones de la columna vertebral de un ejemplar de elasmosáurido proveniente del Miembro Cape Lamb (Campaniano tardío-Maastrichtiano temprano) de la Formación Snow Hill Island, Isla Vega, Península Antártica. Se realizaron tres secciones (anterior, media y posterior) de una vértebra cervical, una dorsal y una caudal y una sección (media) de una vértebra pectoral. Los resultados muestran que las características internas de los elementos estudiados presentan diferencias en cuanto a las estructuras histológicas preservadas, el grado de remodelación y de compactación. Entre algunos de los resultados obtenidos cabe mencionar que las secciones medias de las muestras preservan la mayor cantidad de líneas de crecimiento detenido (Lags). Entre las 4 vértebras analizadas, la vértebra caudal presenta el mayor número de Lags. La vértebra caudal es la que presenta un menor grado de remodelación. Estas diferencias en la microestructura ósea son claramente identificables y brindan un criterio a la hora de determinar qué elemento de la columna y en qué sección del mismo realizar las secciones delgadas. Esto proporcionará una mayor información a nivel paleohistológico que permitirá realizar inferencias de índole paleobiológicas. 


\title{
REGISTRO DE UN CRÁNEO DE UN POSIBLE TEIIDAE (REPTILIA: SQUAMATA) PARA LA FOR- MACIÓN CHICHINALES (MIOCENO TEMPRANO), RÍO NEGRO, PATAGONIA, ARGENTINA
}

\author{
S.L. Garcia ${ }^{1}$, A. Paulina-Carabajal ${ }^{2}$, P. Cruzado-Caballero ${ }^{3}$ y J.D. Daza ${ }^{4 *}$ \\ ${ }^{1}$ Universidad Nacional de Rio Negro, Sede Alto Valle y Valle Medio. Estados Unidos 750, R8332BRJ General Roca, Río Negro, Argentina. \\ sgarcia@unrn.edu.ar \\ ${ }^{2}$ Instituto de Investigaciones en Biodiversidad y Medioambiente (INIBIOMA), Consejo Nacional de Investigaciones Científicas y Tecnológicas- \\ Universidad Nacional del Comahue (CONICET-UNCO). Quintral 1250, R8400FRF San Carlos de Bariloche, Río Negro, Argentina. \\ premjisaurus@yahoo.com.ar \\ ${ }^{3}$ Instituto de Investigación en Paleobiología y Geología (IIPG)-Consejo Nacional de Investigaciones Científicas y Tecnológicas (CONICET), \\ Universidad Nacional de Río Negro. Av. Roca 1242, R8332EXZ Gral. Roca, Río Negro, Argentina.pccaballero@unrn.edu.ar \\ ${ }^{4}$ Department of Biological Sciences, Sam Houston State University. 2000 Avenue I, Life Sciences Building, Room 105, TX 77341 Huntsville, \\ Alabama, Estados Unidos. juand.daza@gmail.com
}

Damos a conocer un cráneo de lagarto parcialmente preservado que presenta características afines a la familia Teiidae (Reptilia: Squamata). El espécimen (número provisorio de campo IIPG-16) fue encontrado dentro del Área Natural Protegida Paso Córdoba, localizada a 12 km al sur de General Roca (Río Negro) en sedimentos pertenecientes a la Formación Chichinales (Mioceno Temprano). Mediante el uso de microtomografía se pudo determinar que el ejemplar conserva parte del techo craneano, nasales, neurocráneo, escamoso, cuadrado, huesos de la órbita, maxilar y mandíbulas inferiores completas. Entre los caracteres que definen a la Familia Teiidae, presentes en el IIPG-16, se observan los siguientes: región preorbital elongada y puntiaguda, parietales no extendidos lateralmente, postfrontal alargado, dentición heterodonta, postorbital bien desarrollado y quadrado con membrana timpánica bien desarrollada. El fósil presenta una meseta del parietal triangular (posterior a la sutura frontoparietal) con bordes posteriores rectos que se continúan en una cresta sagital corta y delgada similar a Callopistes Núñez y Veloso. El registro fósil de esta formación para la zona de Paso Córdoba está constituido principalmente por restos de mamíferos, dos familias de tortugas, restos de reptiles asignables al Orden Crocodilia y un taxón de aves Ralliformes. El nuevo ejemplar es una fuente muy valiosa de información sobre la evolución morfológica y la filogenia de la Familia Teiidae, debido a que los registros más comunes corresponden a fragmentos de mandíbulas y vértebras que solo aportan a la distribución geográfica de los mismos.

*Proyecto subsidiado por PICT 2016-0481 (AP-C, PC-C).

\section{A REVIEW OF THE SOUTH AMERICAN MEMBERS OF THE FAMILY STRUTHIOLARIIDAE FISCHER}

\author{
S. Genta IturRería 1 , And M. GRIFFIN²
}

${ }^{1}$ Facultad de Ciencias Naturales y Museo, Universidad Nacional de La Plata. Av. 122 y 60, B1904 La Plata, Buenos Aires, Argentina. gentaiturreria@gmail.com

2División Paleozoología de Invertebrados. Unidades de Investigación Anexo Museo, Facultad de Ciencias Naturales y Museo, Universidad Nacional de La Plata. Av. 60 y 122 s/n, B1904 La Plata, Buenos Aires, Argentina.miguelgriffin@aol.com

The Family Struthiolariidae includes a number of taxa that lived in the circum-Antarctic region since the Late Cretaceous. A few extant species currently live off the coast of Australia and New Zealand and one species is known from the southwestern Atlantic Ocean. Fossil representatives are common in many Cenozoic stratigraphic units exposed in Australia, New Zealand, Antarctica and South America. Members of this family appear to have been restricted to the high latitudes of the southern hemisphere. Many species are conspicuous members of the shallow marine faunal assemblages of different ages occurring in this area, and there are twelve nominal species described from South America. Yet, in some cases it is difficult to differentiate them, mainly because they have been based a limited number of specimens, preventing to assess properly the variations occurring in the characters used to define each one. New material from different localities in Patagonia reveals that the number of fossil species is in fact not more than nine. These species appear to be restricted to certain stratigraphic units, and thus may be potentially useful for local or regional biostratigraphic purposes. 


\title{
NUEVOS REGISTROS DE XENARTHRA (MAMMALIA) PARA LA FORMACIÓN COLLÓN CURÁ, MIO- CENO DE LA PROVINCIA DEL CHUBUT: MEGATHERICULUS PATAGONICUS AMEGHINO
}

\author{
L.R. González Ruiz¹ , D. BrandonI² y J. BuCher ${ }^{3 *}$ \\ ${ }^{1}$ Centro de Investigación Esquel de Montaña y Estepa Patagónicas (CONICET-UNPSJB), Roca 780, 9200 Esquel, Chubut, Argentina. \\ Irgonzalezruiz@comahue-conicet.gob.ar \\ 2Laboratorio de Paleontología de Vertebrados, Centro de Investigaciones Científicas y Transferencia de Tecnología a la Producción (CICyTTP), \\ Consejo Nacional de Investigaciones Científicas y Tecnológicas (CONICET)-Entre Ríos, Universidad Autónoma de Entre Ríos (UADER). Matteri \\ y España s/n, E3105BWA Diamante, Entre Ríos, Argentina. debrandoni@cicyttp.org.ar \\ ${ }^{3}$ Centro de Investigaciones geológicas, Universidad Nacional de La Plata-Consejo Nacional de Investigaciones Científicas y Tecnológicas \\ (CONICET). Diagonal 113 n²74, B1904DPK La Plata, Buenos Aires, Argentina. joaquinbucher@yahoo.com.ar
}

La fauna de Xenarthra del Mioceno Medio-Tardío (Serravaliano-Tortoniano) de la provincia del Chubut está representada por distintos grupos de Cingulata (e.g., Epipeltephilus Ameghino, Prozaedyus Ameghino, Stenotatus Ameghino, Palaehoplophorus Ameghino) y Tardigrada (e.g., Megathericulus Ameghino, Xyophorus Ameghino). En lo que refiere a tardígrados, en esta contribución se dan a conocer nuevos ejemplares del Megatheriinae Megathericulus patagonicus Ameghino. Los ejemplares consisten en: 1) LIEB PV 6152, un fragmento izquierdo de dentario procedente de la localidad La Gloria, cercana a la localidad de Paso del Sapo, y 2) LIEB PV 6153, un astrágalo derecho procedente de la localidad Cerro Zeballos cercana a la localidad de Gualjaina, ambas ubicadas en el noroeste de la provincia del Chubut. Los ejemplares fueron registrados en sedimentos arenolimosos de composición dominantemente volcaniclástica atribuidos a la Formación Collón Curá (Serravaliano-Tortoniano), acumulados en una variedad de ambientes continentales, desde sistemas aluviales a fluviales e incluso lacustres someros. LIEB PV 6152 presenta un índice de hipsodoncia cercano a 100 y los molariformes son de sección cuadrangular y la superficie oclusal presenta un marcado valle en V. En el astrágalo LIEB PV 6153 la faceta navicular se encuentra dorsal respecto a la faceta discoidal. Megathericulus patagonicus es el Megatheriinae nominado más antiguo con un biocrón que se extiende desde el Serravaliano de Huayllajara, Bolivia (formación innominada del Grupo Honda) hasta el Serravaliano-Tortoniano de las provincias del Chubut y Santa Cruz, Patagonia, Argentina (Formaciones Collón Curá, Arroyo Pedregoso, y Río Mayo).

*Proyecto financiado por PICT 2013-2270.

\section{DEPÓSITOS MARINOS DEL PALEOCENO EN LA CUENCA VALDÉS: QUISTES DE DINOFLAGELADOS Y NANOFÓSILES}

\author{
M.V. Guler ${ }^{1}$, M.S. González Estebenet ${ }^{1}$, J.P. Pérez Panera ${ }^{2}$, E.L. Navarro³ ${ }^{3}$ G.N. Angelozzi ${ }^{4}$, D. Pieroni ${ }^{3}$ y J.P. Lovecchio5*
}

'Instituto Geológico del Sur (INGEOSUR)-Depto. de Geología, Universidad Nacional del Sur (UNS)-Consejo Nacional de Investigaciones Científicas y Tecnológicas (CONICET). San Juan 670, B8000ICN Bahía Blanca, Buenos Aires, Argentina.vvuler@criba.edu.ar; sol.gonzalezestebenet@uns.edu.ar ${ }^{2}$ Laboratorio de Bioestratigrafía, YPF Tecnología S.A.-Consejo Nacional de Investigaciones Científicas y Tecnológicas (CONICET). Avenida del Petróleo Argentino s/n entre 129 y 143, 1923 Berisso, Buenos Aires, Argentina. juan.p.panera@ypftecnologia.com

${ }^{3}$ Departamento de Geología, Universidad Nacional del Sur (UNS). San Juan 670, B8000ICN Bahia Blanca, Buenos Aires, Argentina. enavarro@criba.edu.ar; daniel.pieroni@uns.edu.ar

${ }^{4}$ GEMA S.R.L. - Laboratorio de Bioestratigrafía, YPF Tecnología S.A. Avenida del Petróleo Argentino s/n entre 129 y 143,1923 Berisso, Buenos Aires, Argentina.gladys.angelozzi@set.ypf.com

5YPF S.A. Offshore Exploration. Macacha Güemes 515, C1106BKK Ciudad Autónoma de Buenos Aires, Argentina. juan.lovecchio@ypf.com

La Cuenca Valdés se ubica en el margen Atlántico Sudoccidental y su formación está vinculada a una etapa de rifting entre América del Sur y África durante el Jurásico Medio a Superior. Se analizaron palinomorfos marinos y nanofósiles provenientes del tramo comprendido entre los 778 y 885 mbbp del Pozo onshore YPF-CH-PV.es-1, Península Valdés, provincia de Chubut. Las asociaciones de quistes de dinoflagelados presentan diversidad, abundancia variable y buena preservación. Las últimas ocurrencias de Fibrocysta bipolaris (Cookson y Eisenack) Stover y Evitt, Senoniasphaera inornata (Drugg) Stover y Evitt, Trithyrodinium evittii Drugg, Cerodinium diebelii (Alberti) Lentin y Williams y Disphaerogena carposphaeropsis Wetzel indicaron una edad mínima paleocena (Selandiano). Por otra parte, se identificaron desde la base del intervalo Alisocysta circumtabulata (Drugg) Stover y Evitt, Disphaerogena carposphaeropsis, Glaphyrocysta perforata Hultberg y Malmgren, Deflandrea galeata (Lejeune-Carpentier) Lentin y Williams, Trithyrodinium evittii, Pierceites pentagonus (May) Habib y Drugg y Fibrocysta bipolaris cuyas primeras ocurrencias se registran en el Maastrichtiano tardío. No obstante, los rangos de estas especies atraviesan el limite Maastrichtiano/Daniano y no se identificaron taxa diagnósticos del techo del Maastrichtiano. Los nanofósiles calcáreos presentan fuerte disolución, sin embargo, se pudieron reconocer marcadores del Daniano como Cruciplacolithus tenuis (Stradner) Hay y Mohler, C. primus Perch-Nielsen, Hornibrookina edwardsii Perch-Nielsen y Toweius africanus (Perch-Nielsen) Perch-Nielsen. En su conjunto, estos marcadores indicarían una edad Daniano temprano, biozonas NP2-NP3. Se destaca la gran abundancia de dinoflagelados calcáreos y retrabajo de formas cretácicas, como también ocurre en el Daniano de las cuencas del Colorado y del Salado. 


\title{
GEOMORPHOMETRIC ANALYSIS OF AZYGOGRAPTUS SPECIES (GRAPTOLITHINA) FROM THE CENTRAL ANDEAN BASIN
}

\author{
N.C. Herrera Sánchez ${ }^{1}$, F.J. Degrange ${ }^{1}$, B.A. Toro ${ }^{1}$, and G. Lo Valvo ${ }^{2}$ \\ ${ }^{1}$ Centro de investigaciones en Ciencias de la Tierra (CICTERRA)-Consejo Nacional de Investigaciones Científicas y Técnicas (CONICET)-Uni- \\ versidad Nacional de Córdoba. Av. Vélez Sársfield 1611,X5016CGA Córdoba, Córdoba, Argentina. nexxysherrera@gmail.com; fjdino@gmail.com; \\ btorogr@mendoza-conicet.gov.ar \\ ${ }^{2}$ Facultad de Ciencias Exactas y Naturales, Universidad de Buenos Aires. Intendente Güiraldes 2160, Ciudad Universitaria, C1428EGA, Ciudad \\ Autónoma de Buenos Aires, Argentina. gerardolovalvo@gmail.com
}

The single-stiped morphology and simple thecal form make the genus Azygograptus easily recognizable, but the identification at species level results complicated by subtle differences, the scarcity of detailed descriptions, intraspecific variations and deformation. Here, an elliptic Fourier analysis is carried out in the proximal end of individuals to test the systematic classification at species level. The resulting coordinates were subjected to an exploration using principal components and cluster analysis to examine groupings. We include 27 individuals from two species characterized by the absence of adpressed growth of theca1 along the sicula: Azygograptus lapworthi Nicholson and A. eivinonicus Elles. Most of the material comes from the Central Andean basin (northwestern Argentina and southern Bolivia), but specimens from South China, Scandinavia and Great Britain are also included for comparison. The contour analysis showed that the first three components explain $79.21 \%$ of the total variation in the shape of the specimens. PC1 represented the stipe width at theca 1 and the differences in the sicular morphology: narrow stipes at theca1 and large-elongated siculas (negative) vs. wide stipes at theca 1 and short-stouter siculas (positive). Meanwhile, PC2 explained the variation in the stipe divergence angle: close to $90^{\circ}$ (negative) vs. greater than $90^{\circ}$ (positive). Moreover, dendrograms illustrated that individuals from different species are related and grouped together. Thus, the resulting morphospaces and clusterings allow inferring that the specimens previously assigned as two different species are geomorphometrically indistinguishable. Therefore, we can conclude that all the material could correspond to a unique species ( $A$. lapworthi) with intraspecific variations.

*Financial support by Agencia Nacional de Promoción Científica y Tecnológica (ANPCYT) PICT 2016-0558, IUGS-IGCP Project 653 'The onset of the Great Ordovician Biodiversification Event'.

\section{ASOCIACIONES DE HELEOBIA AMEGHINI DOERING (GASTROPODA) Y OSTRACODA (CRUSTACEA) EN EL PLEISTOCENO TARDÍO DEL CENTRO ESTE DE LA REGIÓN PAMPEANA. CONSIDERACIONES PALEOAMBIENTALES}

\author{
F. IACONA ${ }^{1}$ Y A.P. CARIGNANO ${ }^{2 *}$
}

${ }^{1}$ Agencia Nacional de Promoción Científica y Tecnológica (ANPCyT). División Paleontología Vertebrados, Museo de La Plata, Facultad de Ciencias Naturales y Museo, Universidad Nacional de La Plata. Paseo del Bosque s/n, B1900FWA La Plata, Buenos Aires, Argentina. facundoiacona@fcnym.unlp.edu.ar

${ }^{2}$ Consejo Nacional de Investigaciones Científicas y Tecnológicas (CONICET). División Paleozoología Invertebrados, Museo de La Plata, Facultad de Ciencias Naturales y Museo, Universidad Nacional de La Plata, Paseo del Bosque s/n, B1900FWA La Plata, Buenos Aires, Argentina. apcarignano@fcnym.unlp.edu.ar

Abundantes especímenes del gasterópodo Heleobia Stimpson y una diversa asociación de ostrácodos junto a un cráneo completo de Macrauchenia patachonica Owen, fueron recuperados en la "Reserva paleontológica Marcos Paz" (Cantera "Nicolás Vignogna III", partido de Marcos Paz, Provincia de Buenos Aires). Análisis cualitativos y cuantitativos de los ejemplares de Heleobia, permitieron su asignación preliminar a Heleobia ameghini, fósil guía de Edad Lujanense. La asociación de ostrácodos resultó diversa y abundante, registrándose Cyprideis salebrosa hartmanni Ramírez, Eucypris virens Jurine, Limnocythere cusminskyae Ramón-Mercau, Plastani y Laprida, Heterocypris incongruens Ramdhor, Cypridopsis vidua Müller, y en menor medida Chlamydotheca incisa Claus, Ilyocypris ramirezi Cusminsky y Whatley, Limnocythere sp. y Candona sp. En todos los casos, se encontraron ejemplares adultos y juveniles de diversos estadios. Si bien C. salebrosa hartmanni es la especie con mayor representatividad en la asociación, dos cuestiones deben tenerse en cuenta al hacer inferencias paleoambientales: 1) en general, los ostrácodos no marinos están pobremente calcificados, así resultan más susceptibles a la fragmentación incluso durante el lavado de la muestra, por lo que pueden estar subrepresentados (e.g., E. virens), al contrario de C. salebrosa hartmanni (comúnmente más calcificados); y 2) un gran número de juveniles de C. salebrosa hartmanni aparecen con dos tubérculos posteriores y fuertemente reticulados, caracteres probablemente asociados a la condición del cuerpo de agua. Datos aportados por la sedimentología y la microfauna permiten inferir como paleoambiente de depositación un cuerpo de agua canalizado, hipohalino-oligohalino, permanente/semipermanente, somero, vegetado, bien oxigenado y de baja energía.

*Proyecto financiado por PICT 2016-2698, PICT 2014-1964. 


\title{
MACRAUCHENIA PATACHONICA (LITOPTERNA: MACRAUCHENIIDAE) EN EL PLEISTOCENO TARDÍO DEL CENTROESTE DE LA REGIÓN PAMPEANA (ARGENTINA). APORTES BIOESTRATIGRÁFICOS Y PALEOAMBIENTALES
}

\author{
F. IACONA ${ }^{1,2}$, E. SolbelzON ${ }^{1,3}$, L.H. SOIBELZON ${ }^{1,3}$ y S.G. RodRIGUEZ ${ }^{4 *}$ \\ 'División Paleontología Vertebrados, Museo de La Plata, Facultad de Ciencias Naturales y Museo, Universidad Nacional de La Plata. Paseo del \\ Bosque s/n, B1900FWA La Plata, Buenos Aires, Argentina.facundoiacona@fcnym.unlp.edu.ar, esoibelzon@fcnym.unlp.edu.ar; Isoibelzon@fcnym.unlp.edu.ar \\ ${ }^{2}$ Agencia Nacional de Promoción Científica y Tecnológica (ANPCyT). \\ ${ }^{3}$ Consejo Nacional de Investigaciones Científicas y Tecnológicas (CONICET). \\ ${ }_{4}^{4}$ Facultad de Ciencias Naturales y Museo, Universidad Nacional de La Plata. Av. 122 y60, B1904 La Plata, Buenos Aires, Argentina. srodriguez@fcnym.unlp.edu.ar \\ Esta contribución tiene por objetivo dar a conocer nuevos restos asignados a Macrauchenia patachonica Owen, proceden- \\ tes del yacimiento "Cantera Nicolás Vignogna III" (Reserva Paleontológica "Francisco P. Moreno", partido de Marcos Paz, \\ Buenos Aires), ubicado en el ámbito geológico de la llanura Chaco-Bonaerense. Se colectó un cráneo completo, vértebras, \\ mandíbulas, molares y otros restos postcraneales (repositorio Museo Paleontológico Lucas Kraglievich, MPLK). Los mate- \\ riales provienen de dos asociaciones de facies: una corresponde a canales de baja jerarquía (como arroyos costeros que ex- \\ perimentaron desbordes durante periodos de alta descarga) y la otra al relleno de canales de baja sinuosidad y baja movi- \\ lidad (FA2 y FA4, respectivamente). Estudios previos de este equipo de investigación indican que de ambas facies se \\ colectaron restos de Heleobia sp. (Gastropoda), que arrojaron edades de 31040 +/- 740 (FA2) y 29070 +/- 1420 años AP \\ (FA4), lo que ubica la secuencia en el Pleistoceno Tardío. A su vez, la composición faunística que acompaña estos restos se \\ corresponde con la Biozona de Equus (Amerhipuus) neogeus, de Edad Lujanense. De los materiales de M. patachonica, se des- \\ taca uno por su excelente calidad de preservación, y corresponde a un cráneo y mandíbula (MPLK-0005, exhumado de FA4). \\ El sedimento portador contiene abundantes restos de ostrácodos, peces y gasterópodos, lo que sugiere un cuerpo de agua \\ hipo-oligohalina, de baja energía y vegetado. Los gasterópodos hallados en el sedimento portador del ejemplar MPLK- \\ 0005, fueron determinados como Heleobia ameghini Doering, fósil guía del Lujanense, reafirmando su asignación temporal, \\ que será contrastada con nuevas dataciones sobre estos gasterópodos.
}

*Proyecot financiado por PICT 2016-2698; PI N-733 UNLP.

\section{PALEOSUELOS Y TRAZAS FÓSILES: BIOLOGÍA DE SUELOS PASADOS EN DEPÓSITOS FLUVIALES DE LA FORMACIÓN CANDELEROS (GRUPO NEUQUÉN), CENTRO-OESTE DE RÍO NEGRO}

\author{
J. Kaluza ${ }^{1}$, M.S. Gualde², M.L. Sánchez ${ }^{2}$ y S. Apesteguía ${ }^{1 *}$ \\ ${ }^{1}$ Consejo Nacional de Investigaciones Científicas y Tecnológicas (CONICET). Fundación de Historia Natural Félix de Azara, Centro de Estudios \\ Biomédicos, Biotecnológicos, Ambientales y Diagnóstico (CEBBAD)-Universidad Maimónides. Hidalgo 775, C1405CBK Ciudad Autónoma de \\ Buenos Aires, Argentina.yojonatan@hotmail.com; sebapesteguia@gmail.com \\ ²Departamento de Geología, Universidad Nacional de Río Cuarto. Ruta Nac. 36, Km. 601, 5800 Río Cuarto, Córdoba, Argentina. \\ msoledadgualde@gmail.com; msanchez@exa.unrc.edu.ar
}

En el presente trabajo se dan a conocer los resultados del estudio de paleosuelos y la actividad biológica relacionada, en facies de areniscas fluviales del tramo superior de la Formación Candeleros (Cenomaniano), en Cuenca Neuquina. Los sistemas fluviales están asociados a periodos de alta descarga de agua y sedimentos que desarrollaron extensos abanicos de desbordes. El perfil tipo de paleosuelo mide 0,5-0,7 m y es de textura es arcillosa-limosa; el color oscila entre amarillo-naranja claro y marrón rojizo; exhibe grietas bien desarrolladas con 3-0,5 cm de abertura y 10-20 cm de profundidad. Respecto de las trazas fósiles, presenta excavaciones sub-verticales de $30 \mathrm{~cm}$ de longitud y 4 cm de diámetro, de techo cóncavo y base plana, ornamentadas con marcas de garras; también se encontró un sistema de excavaciones, formado por cámaras y túneles verticales con uniones simples, de hasta $40 \mathrm{~cm}$ de largo. Asimismo, exhibe rizolitos tubulares de hasta $20 \mathrm{~cm}$ de largo y $4-8 \mathrm{~cm}$ de diámetro; junto a estos se encontraron estructuras de nidificación de insectos preservadas como moldes ovoides de $\sim 3 \mathrm{~cm}$ de largo y 1 cm de ancho, de calcita y fango arcilloso. Los paleosuelos pertenecen al Orden de los Argilisoles vérticos cálcicos. El tipo de paleosuelo, sus rasgos morfológicos y la naturaleza del registro de actividad biológica, son compatibles con un clima con marcada estacionalidad. Según los modelos climáticos generados para el Cretácico Superior, se esperaría un sistema biológico de estas características evolucione en un bioma subtropical, con clima árido, altamente estacional.

*Proyecto subsidiado por MinCyT PICT 2014-0564 "Biota y ambientes cretácicos del área paleontológica de La Buitrera (Cerro Policía, Río Negro)" Director: Dr. Sebastián Apesteguía. 


\title{
PROSPECCIÓN Y EXTRACCIÓN DE NUEVOS VERTEBRADOS FÓSILES DE LA FORMACIÓN DORO- TEA (CRETÁCICO SUPERIOR), VALLE DEL RÍO DE LAS CHINAS, REGIÓN DE MAGALLANES, CHILE
}

\author{
J. Kaluza1', S. Soto Acuña², L. Manríquez³, R. Otero², R. Fernandez Guimenez, B. Aravena², F. Suazo Lara², J. Alarcon Muñoz², \\ V. MILLA ${ }^{4}$, J.P. PINO ${ }^{5}$ Y H. ORTIZ ${ }^{5 *}$
}

${ }^{1}$ Consejo Nacional de Investigaciones Científicas y Tecnológicas (CONICET). Fundación de Historia Natural Félix de Azara, Centro de Estudios Biomédicos, Biotecnológicos, Ambientales y Diagnóstico (CEBBAD)-Universidad Maimónides. Hidalgo 775, C1405CBK Ciudad Autónoma de Buenos Aires, Argentina.yojonatan@hotmail.com

2Universidad de Chile. Las Palmeras 3425, 7800003 Ñuñoa, Santiago de Chile, Chile. arcosaurio@gmail.com; otero2112@gmail.com;

barbara.aravena.b@gmail.com; felipe.suazo.lara@gmail.com; jhoalarc@gmail.com

3Universidad do Vale do Rio dos Sinos. Av. Unisinos 950, 93020 Cristo Rei, São Leopoldo, Brasil. less.manriquez@gmail.com

«Universidad de Concepción. Víctor Lamas 1290,160-C Concepción, Región del Bío Bío, Chile. rofer87@gmail.com; vero.millaflores@gmail.com ${ }^{5}$ Instituto Antartico Chileno. Plaza Muñoz Gamero 1055, Punta Arenas, Región de Magallanes y de la Antártica Chilena, Chile. juanpablo.pinomorales@gmail.com; hctros@gmail.com

La Formación Dorotea es una unidad rocosa del Cretácico Superior (Campaniano superior-Maastrichtiano) que aflora en el margen nororiental de la provincia de Última Esperanza, región de Magallanes. En varios yacimientos de esta unidad, caracterizada por un sistema de deltas y ríos de meandros, se han preservado un extenso registro faunístico debido a la dinámica fluvial que este tipo de sistemas hídricos poseen. En Febrero del 2018, un equipo multidisciplinario llevó a cabo el descubrimiento de un nuevo ornitópodo en la Formación Dorotea, particularmente, en el sitio informalmente denominado como "Saurópodo", en ausencia de toponimias para la zona. El ejemplar recuperado se halló parcialmente expuesto con degradación de la superficie por la continua erosión del ambiente, presentando un gran porcentaje de sus elementos óseos en desarticulación, mientras que las extremidades traseras se hallaron parcialmente articuladas. Por otro lado, se realizó la prospección y extracción de pequeños vertebrados fósiles en las cercanías del hallazgo anterior. Debido a que el nivel portador se halla en extrema erosión por la continua exposición a los agentes de meteorización, los fósiles allí hallados presentan una asociación de individuos en desarticulación masiva, encontrándose depositados en niveles inferiores y no in situ. Cuando una asociación multiespecífica de individuos es descubierta sobre un mismo estrato o plano de depositación, se deben desarroIlar estrategias especiales para su colecta. Es por eso que este trabajo intenta exponer brevemente las diversas estrategias de prospección, extracción y logística utilizadas en la colecta de especímenes en las localidades antes mencionadas.

*Proyecto subsidiado por Fondecyt N¹151389 y Proyecto Anillo ACT-172099.

\section{TÉCNICAS DE EXTRACCIÓN Y PROCEDIMIENTOS QUÍMICO-MECÁNICOS APLICADO A FÓSILES CONSERVADOS EN ASFALTO, RANCHO LA BREA TAR PITS \& PAGE MUSEUM (CALIFORNIA, ESTADOS UNIDOS)}

\section{J. KALUZA ${ }^{1 *}$}

${ }^{1}$ Consejo Nacional de Investigaciones Científicas y Tecnológicas (CONICET). Fundación de Historia Natural Félix de Azara, Centro de Estudios Biomédicos, Biotecnológicos, Ambientales y Diagnóstico (CEBBAD)-Universidad Maimónides. Hidalgo 775, C1405CBK Ciudad Autónoma de Buenos Aires, Argentina. yojonatan@hotmail.com

Durante unos 50.000 años, los pozos han emitido una gran cantidad de asfalto espeso y pegajoso que ha atrapado a muchos especímenes de plantas, insectos y vertebrados prehistóricos, resultando en uno de los más prolíficos depósitos de organismos de la Edad de Hielo en el mundo. Los fósiles de esta localidad presentan un magnifico estado de preservación manteniendo su composición orgánica inalterada, es decir, que a diferencia de otros fósiles de edades similares hallados alrededor del mundo, estos no han sufrido el proceso de permineralización. Esto se debe principalmente a la impregnación de asfalto que los huesos poseen, que ayuda a proteger el material de los cambios diagenéticos. El objetivo principal de esta contribución es dar a conocer información disponible sobre las distintas técnicas de extracción y procedimientos utilizados en la recopilación de datos biológicos in situ como las metodológicas químicas/mecánicas llevadas a cabo en laboratorio. Los fósiles preservados en asfalto, requieren mecanismos específicos de extracción por lo cual se utilizan técnicas metódicas que permiten muestrear intensamente y registrar cuidadosamente los datos biológicos y geológicos preservados. Uno de los objetivos principales de las excavaciones, es la recuperación de los fósiles de microvertebrados, estos han demostrado que brindan una gran cantidad de información sobre los ambientes y ecosistemas del pasado. La preparación final de los especímenes colectados es realizada por medio de agentes químicos y procesos manuales que permiten disolver la matriz exponiendo así las características anatómicas que los fósiles colectados poseen y son esenciales para su posterior estudio.

*Proyecto financiado por Natural History Museum of Los Angeles County y Dinosaur Institute. 


\title{
MAMÍFEROS FÓSILES DE LA FORMACIÓN LA PAVA (MIOCENO INFERIOR-MEDIO) EN LA REGIÓN DE INGENIERO JACOBACCI, PROVINCIA DE RÍO NEGRO
}

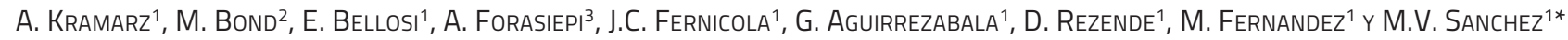 \\ ${ }^{1}$ Museo Argentino de Ciencias Naturales "Bernardino Rivadavia" (MACN)-Consejo Nacional de Investigaciones Científicas y Tecnológicas \\ (CONICET). Av. Ángel Gallardo 470, C1405DJR Ciudad Autónoma de Buenos Aires, Argentina.agkramarz@macn.gov.ar; beledu00@gmail.com; \\ jctano@macn.gov.ar; guillecomallo@yahoo.com.ar;dtrezende@macn.gov.ar;Mechi_985@hotmail.com; maviksan@gmail.com \\ ${ }^{2}$ Consejo Nacional de Investigaciones Científicas y Tecnológicas (CONICET). División Paleontología de Vertebrados, Museo de La Plata, Facul- \\ tad de Ciencias Naturales y Museo, Universidad Nacional de La Plata. Paseo del Bosque s/n, B1900FWA La Plata, Buenos Aires, Argentina. \\ constantino1453@yahoo.com.ar \\ ${ }^{3}$ Instituto Argentino de Nivología, Glaciología y Ciencias Ambientales (IANIGLA), Centro Científico Tecnológico Mendoza-Consejo Nacional de \\ Investigaciones Científicas y Tecnológicas (CONICET). Avenida Ruiz Leal s/n, 5500 Mendoza, Mendoza, Argentina.borhyaena@hotmail.com
}

La Formación La Pava (FLP) se distribuye en cuencas intermontanas del antepaís neógeno norpatagónico (Chubut, Río Negro, Neuquén). Los diferentes depósitos (chonitas, tufitas, conglomerados) están modificados en paleosuelos con abundantes trazas fósiles. Le suceden la Formación Collón Curá (FCC), portadora de una rica asociación de mamíferos que caracteriza la Edad Mamífero Colloncurense (Mioceno Medio). Antiguamente la FLP era considerada una subunidad de la FCC, por lo que resulta incierto si los fósiles de antiguas colecciones referidos a la FCC provenían de esta o de la FLP. Recientes prospecciones en la FLP al Sur del valle del arroyo Huahuel Niyeu (Ea. Huanuluan, $33 \mathrm{~km}$ al oeste de Ingeniero Jacobacci), permitieron identificar un conjunto de mamíferos representado por Proeutatus, Prozaedyus, Glyptodontidae (Cingulata), Palaeothentes (Paucituberculata), Astrapotherium? (Astrapotheria), y dos especies de Toxodontidae (Notoungulata) (depositados en el Museo J. G. Gerhold de Ing. Jacobacci). Los toxodóntidos presentan rasgos dentarios más avanzados que sus contrapartes santacrucenses (dientes de corona más alta, molares superiores con valle lingual abierto e inferiores sin fosétidas de esmalte), comparables a los que caracterizan la fauna colloncurense. Además, los holotipos de Massoiamys obliquus Vucetich (Rodentia) y Glossotheriopsis pascuali Scillato-Yané (Tardigrada) provendrían de los mismos niveles expuestos en Ea. Los Sauces, 1-2 km al oeste de la localidad anterior. La presencia de taxones característicos de la Edad Colloncurense en la FLP indica que su advenimiento en Norpatagonia podría remontarse al menos hasta el Mioceno Temprano terminal y seguramente antecede a la depositación de la FCC.

*Proyecto financiado por PIP 0725/2014; PICT 0326/2012; PIP 0058/2013.

\section{MAMÍFEROS PRE-COLLONCURENSES DE LA LAGUNA CARRI LAUFQUEN CHICA, SUDOESTE DE LA PROVINCIA DE RÍO NEGRO}

\author{
A. Kramarz ${ }^{1}$, M. Bond², E. Bellosi ${ }^{1}$, A. Forasiepi ${ }^{3}$, J.C. Fernicola ${ }^{1}$, G. Aguirrezabala ${ }^{1}$ y D. Rezende ${ }^{1}$
}

${ }^{1}$ Museo Argentino de Ciencias Naturales "Bernardino Rivadavia" (MACN)-Consejo Nacional de Investigaciones Científicas y Tecnológicas (CONICET). Av. Ángel Gallardo 470, C1405DJR Ciudad Autónoma de Buenos Aires, Argentina.agkramarz@macn.gov.ar; beledu00@gmail.com; jctano@macn.gov.ar; guillecomallo@yahoo.com.ar; dtrezende@macn.gov.ar

${ }^{2}$ Consejo Nacional de Investigaciones Científicas y Tecnológicas (CONICET).. División Paleontología de Vertebrados, Museo de La Plata, Facultad de Ciencias Naturales y Museo, Universidad Nacional de La Plata. Paseo del Bosque s/n, B1900FWA La Plata, Buenos Aires, Argentina. constantino1453@yahoo.com.ar

${ }^{3}$ Instituto Argentino de Nivología, Glaciología y Ciencias Ambientales (IANIGLA), Centro Científico Tecnológico Mendoza-Consejo Nacional de Investigaciones Científicas y Tecnológicas (CONICET). Avenida Ruiz Leal s/n, 5500 Mendoza, Mendoza, Argentina. borhyaena@hotmail.com

Las referencias disponibles sobre sedimentos con mamíferos más antiguos que los de la Formación Collon Curá (FCC, Mioceno Medio) en la vertiente occidental del macizo de Somuncurá son imprecisas y contradictorias. La mayoría de estas se concentra en el área de Ing. Jacobacci (SO de la provincia de Río Negro), originadas en los trabajos pioneros de R. Casamiquela durante los años '50 y '60. Recientes trabajos de prospección en el cerro Ambrosio, al N de la Laguna Carri Laufquen Chica, revelaron la existencia de restos asignables a Parastrapotherium (Astrapotheria) y Cochilius (Notoungulata) (depositados en el Museo J.H. Gerhold), que sugieren un rango de edad Deseadense (Oligoceno Tardío) a Colhuehuapense (Mioceno Temprano), confirmando la existencia en el área de depósitos portadores de mamíferos significativamente más antiguos que Mioceno Medio. Los restos provienen de la sección media de una sucesión (30 m) piroclástica, bien estratificada y blanquecina que sobreyace en discordancia la Formacion Coli-Toro (Cretácico) y está cubierta discordantemente por la Formación La Pava (Mioceno inferior?). La sucesión fosilífera incluye tufitas, chonitas y paleosuelos que registran sedimentación en ambientes fluviales y lacustres. Muy probablemente, el único cráneo asignado al controvertido cingulado paleógeno Paleopeltis provenga de estos mismos nivel y localidad, así como restos asignables a Proadinotherium (Notoungulata) depositados en el MLP. Estos depósitos fueron antiguamente mapeados como parte de la FCC, por lo que es muy probable que materiales de antiguas colecciones en el área de Ing. Jacobacci referidas a la FCC provengan de estos u otros niveles equivalentes expuestos en la región. 


\title{
PRESERVED CHEMISTRY OF PTILOPHYLLUM FRONDS FROM THE SPRINGHILL FORMATION (LOWER CRETACEOUS, ARGENTINA)
}

\author{
M.A. Lafuente Diaz ${ }^{1}$, M.A. Carrizo ${ }^{1}$, G.M. Del Fueyo ${ }^{1}$, and J.A. D’angelo ${ }^{2 *}$ \\ 'Museo Argentino de Ciencias Naturales "Bernardino Rivadavia" (MACN)-Consejo Nacional de Investigaciones Científicas y Tecnológicas \\ (CONICET). Av. Ángel Gallardo 470, C1405DJR Ciudad Autónoma de Buenos Aires, Argentina.maitenlafuentediaz@gmail.com; \\ blackdisk@gmail.com; georgidf@yahoo.com.ar \\ 2Instituto Argentino de Nivología, Glaciología y Ciencias Ambientales (IANIGLA), Centro Científico Tecnológico Mendoza-Consejo Nacional de \\ Investigaciones Científicas y Tecnológicas (CONICET). Avenida Ruiz Leal s/n, 5500 Mendoza, Mendoza, Argentina. joseadangelo@yahoo.com
}

Fronds of a new species of Ptilophyllum Harris (Bennettitales) are analyzed for the first time by semi-quantitative Fourier Transform Infrared (FTIR) spectroscopy with the aim of revealing mesophyll and cuticle chemical structures (functional groups) preserved in different frond parts (apical, medium, basal) as well as their relation with kerogen types. Fossils are compressions with well-preserved cuticles (MPM-PB 15355) found in the Springhill Formation (Hauterivian-Barremian) at the Río Correntoso Locality, Santa Cruz. For the spectrometric study, fronds parts were analyzed into two sample forms: (1) compressions (including coalified mesophyll and cuticle) and (2) cuticles. Semi-quantitative IR-data were evaluated using PCA showing the behavior of the analyzed categories according to the functional groups. In order to distinguish the existence of differences depending on the frond parts an ANOVA was carried out using the two first PCs data. The results indicate that compressions have a similar chemical composition while cuticles show a greater variability. In the first case, the mesophyll may hide cuticular differences between samples. In the second case, the variation mainly reflected on oxygen-containing compounds may be due to the cuticle chemical composition and its transformation through the fossilization process. Also, the cuticle samples show a higher aromatic carbon contribution than the compressions. The latter can be related to the cuticle biopolymer and its monomers. Additionally, no difference was detected by ANOVA analysis depending on the frond zone for both sample forms. Furthermore, the sample forms have a general chemical composition similar to Type Ilkerogen related to cuticles, spores, and resins.

*Financial support provided by ANPCyT -PICT 528/2012 and 2015-2206, CONICET PIP 2012/212.

\section{A NEW SPECIES OF GENUS ALICHOVIA GORJANSKY FROM THE PONÓN TREHUÉ FORMATION, MENDOZA PROVINCE, ARGENTINA}

\section{F.J. LAVIÉ ${ }^{1}$}

${ }^{1}$ Centro de investigaciones en Ciencias de la Tierra (CICTERRA)-Consejo Nacional de Investigaciones Científicas y Técnicas (CONICET) and Universidad Nacional de Córdoba. Av. Vélez Sársfield 1611, X5016CGA, Córdoba, Córdoba, Argentina. fernandolavie@gmail.com

The Ponón Trehué Formation is a sedimentary succession recognized in the San Rafael Block in Mendoza province. It is composed entirely by carbonate rocks such as sparitic dolomites, microbialitic limestones and nodular limestones at the base and middle part of the unit, while levels of packstone/grainstone intercalated with mudstone/wackestone characterize the upper part. Recently, a high diverse fauna of linguliform brachiopods of the orders Lingulida, Acrotretida, Paterinida and Siphonotretida was described by Holmer, Popov, Lehnert and Ghodabi Pour. The last order mainly characterized by valves with different arrangement of hollow spines and larval and postlarval shell lacking pitted microornamentation. The only material described by these authors that belongs to the siphonotertids was assigned with doubts to Multispinula Rowell. Besides finding a similar fauna in new samples analyzed, a new species of Alichovia is here proposed, being its diagnostic character the presence of bifurcated (ramispinose) spines. Alichovia n. sp. is characterized by a concave shell with spaced concentric lamellae, bearing fine spines in the edges, alternating with thicker multibifurcated spines. Originally, this genus was reported from Baltica (Lower Ordovician of Poland and Middle Ordovician of Estonia and Czech Republic) and later in the eastern margin of Gondwana (Middle Ordovician of Iran). This new species represents the first record of this striking genus in the western margin of Gondwana in the Middle Ordovician. 
Reunión de Comunicaciones de la Asociación Paleontológica Argentina, Puerto Madryn, 2018

\title{
PELVIS AND HIND LIMB MUSCLES RECONSTRUCTION IN THE WELL PRESERVED PSEUDO- SUCHIAN BATRACHOTOMUS KUPFERZELLENSIS
}

\author{
A. LECUONA ${ }^{1 *}$ \\ ${ }^{1}$ Instituto de Investigación en Paleobiología y Geología (IIPG)-Consejo Nacional de Investigaciones Científicas y Tecnológicas (CONICET), Uni- \\ versidad Nacional de Río Negro. Av. Roca 1242, R8332EXZ Gral. Roca, Río Negro, Argentina. alecuona@unrn.edu.ar
}

Most fossil vertebrates are known by their hard-tissues, but inferring their soft-tissues is important to elaborate a bigger picture of them. Muscular reconstructions in fossil vertebrates are mostly focused on mammals and dinosaurs, whereas two such studies were recently performed within the crocodilian-lineage of archosaurs, in the pseudosuchians Poposaurus and Prestosuchus. The most accurate methodology for soft-tissue reconstruction is the EPB, combining phylogeny, osteological correlates, and living relatives comparison. The pelvis and hindlimb musculature of the exquisitely preserved pseudosuchian Batrachotomus is presented here. Most muscles were inferred with low speculation; in more than one third of the attachments, the inferences were the least speculatives (e.g., origins of Mm. ambiens, iliofibularis, gastrocnemius); other muscles present more speculation because the avian homologue is quite different, but their reconstruction is based on similarities with crocodilians (e.g., Mm. puboischiotibialis, fibulares). The lower leg and foot soft-tissues inferences are very speculative, including some muscles unable to reconstruct (e.g., digit flexors and extensors) due to the scarce information and the broad differences with birds. Differences in some muscles like iliotibiales and iliofemoralis origins and puboischiofemoralis attachments were noted with Poposaurus, but shearing the same condition with Prestosuchus. Similarities between Batrachotomus and Prestosuchus are expected by their close phylogenetic affinity and also probably by their quadrupedal locomotion and associated paleobiological traits, contrasting with the biped poposauroid Poposaurus. This muscular reconstruction will allows us to face other studies (e.g., morphofunctional) relevant to understand Batrachotomus paleobiology, and eventually study the evolutionary patterns of the stance and gait within Pseudosuchia.

*Financial support provided by the German Academic Exchange Service (DAAD), hosted by Rainer Schoch (Naturkunde Museum Stuttgart).

\section{EFFECT OF THE GENERALIZED EUCLIDEAN DISTANCE ON DISPARITY ANALYSES OF MORPHO- LOGICAL MATRICES}

\author{
O.E.R. Lehmann ${ }^{1}$, M.D. EzCurRa ${ }^{1}$, R.J. ButLer² ${ }^{2}$ And G.T. Lloyd ${ }^{3}$
}

\begin{abstract}
'Sección Paleontología de Vertebrados, Museo Argentino de Ciencias Naturales "Bernardino Rivadavia" (MACN)-Consejo Nacional de Investigaciones Científicas y Tecnológicas (CONICET). Av. Ángel Gallardo 470, C1405DJR Ciudad Autónoma de Buenos Aires, Argentina. lehmanncxii@gmail.com; martindezcurra@yahoo.com.ar

${ }^{2}$ School of Geography, Earth and Environmental Sciences, University of Birmingham. B15, 2T T, Edgbaston, Birmingham, England. r.butler.1@bham.ac.uk

${ }^{3}$ School of Earth and Environment, University of Leeds. LS2, 9JY, Leeds, England. graemetlloyd@gmail.com
\end{abstract}

A large number of palaeontological studies dealing with morphological disparity has been published over the last decade. A critical step of these studies is the transformation of the morphological matrix into a distance matrix. The generalized Euclidean distance (GED) is the most extensively used distance measure to do this, in part because it allows the use of matrices with high amounts of missing data without the need to remove taxa if a subsequent ordination of the data set is desired to reduce dimensionality. The GED accomplishes this by replacing the missing dissimilarities with a mean weighted dissimilarity. Previous studies suggested that the GED may generate a bias in the morphospace and in some disparity measures, but a detailed analysis of this effect was lacking. By studying over 150 morphological matrices, we find that the GED creates a systematic bias, whereby taxa with higher percentages of missing data are placed closer to the centre of the morphospace than those with more complete scorings. This bias extends into pre- and post-ordination calculations of disparity measures and can lead to erroneous interpretations of disparity patterns, especially if specimens present in a particular time interval or clade have distinct percentages of missing data. Results recovered using an alternative distance measure, Maximum Observed Rescaled Distance (MORD), are more robust to the presence of missing data. This is possibly because MORD does not replace the missing dissimilarities with a mean value. We recommend against using the GED for matrices with a high amount of missing data. 


\title{
FLORA APTIANA (GRUPO BAQUERÓ, SANTA CRUZ) EN EL ÁREA DE BAJO GRANDE, DATOS PALINOLÓGICOS, MESO Y MEGAFÓSILES: UN ESTUDIO INTEGRADO
}

\author{
M. LloRens ${ }^{1}$, V.S. Perez Loinaze², M.G. PAssalia ${ }^{3}$ Y E.I. Vera² \\ 'Laboratorio de Botánica, Facultad de Ciencias Naturales, Universidad Nacional de la Patagonia San Juan Bosco. 9 de Julio 25, U9100CKN \\ Trelew, Chubut, Argentina.magdalena.llorens@gmail.com \\ 2Museo Argentino de Ciencias Naturales "Bernardino Rivadavia" (MACN)-Consejo Nacional de Investigaciones Científicas y Tecnológicas \\ (CONICET). Av. Ángel Gallardo 470, C1405DJR Ciudad Autónoma de Buenos Aires, Argentina. loinazev@gmail.com; evera@macn.gov.ar \\ ${ }_{3}^{3}$ Instituto de Investigaciones en Biodiversidad y Medioambiente (INIBIOMA), Consejo Nacional de Investigaciones Científicas y Tecnológi- \\ cas-Universidad Nacional del Comahue (CONICET-UNCO). Quintral 1250, R8400FRF San Carlos de Bariloche, Río Negro, Argentina. \\ passaliam@gmail.com
}

La flora del Grupo Baqueró es una de las asociaciones vegetales cretácicas mundialmente más conocidas, y Bajo Grande una de sus localidades clásicas, estudiada por más de cinco décadas y reconocida por la calidad de preservación de sus restos fósiles. Se recuperaron de esta área una gran variedad de megafósiles, incluyendo estructuras reproductivas con polen asociado. En esta contribución se reporta el estudio sistemático detallado de una variada asociación polínica. A ella están asociados un conjunto de fragmentos de cutículas, como así también leños carbonizados. Se suman además datos previos y nuevos de megaflora, en un estudio integrado donde se establece el escenario en el que se desarrolló esta flora durante el Aptiano superior. A partir de tres niveles fosilíferos estratigráficamente identificados, correspondientes a la Formación Anfiteatro de Ticó, se obtuvieron cinco muestras palinológicas, en las que se identificaron 130 taxones de esporas y polen que representan un amplio espectro de organismos (algas, hongos, briofitas y plantas vasculares). De los mismos niveles fosilíferos se obtuvieron restos megaflorísticos, y los mesofósiles representados por numerosos restos cuticulares que fueron agrupados en 12 morfotipos. Mega y mesofósiles son comparados con taxones previamente reconocidos en la unidad. Las tres fuentes de información (palinomorfos, fragmentos cuticulares, e improntas) son analizadas en conjunto, integrando además datos sedimentológicos, permitiendo una caracterización más completa de esta flora aptiana de Patagonia.

\section{PRODUCĆIÓN CIENTÍFICA EN GRAPTOLITOS DEL NOROESTE ARGENTINO (NOA): ANÁLISIS BIBLIOMÉTRICO}

\author{
G. Lo Valvo ${ }^{1}$, N. Herrera SÁnchez ${ }^{2}$ y B. Toro ${ }^{2 *}$
}

${ }^{1}$ Facultad de Ciencias Exactas y Naturales, Universidad de Buenos Aires. Intendente Güiraldes 2160, C1428EGA Ciudad Autónoma de Buenos Aires, Argentina. gerardolovalvo@gmail.com

${ }^{2}$ Centro de investigaciones en Ciencias de la Tierra (CICTERRA)-Consejo Nacional de Investigaciones Científicas y Técnicas (CONICET) and Universidad Nacional de Córdoba. Av. Vélez Sársfield 1611, X5016CGA Córdoba, Córdoba, Argentina. nexxysherrera@gmail.com;

btorogr@mendoza-conicet.gov.ar

Desde 1883, año en que Brackebusch reconoció por primera vez la presencia de graptolitos "didymográptidos" en la provincia de Salta, se han publicado 94 trabajos sobre graptolitos del NOA. En algunos de ellos, este grupo biológico constituye una parte fundamental y en otros se utilizó para calibrar registros de distintos grupos fósiles. El objetivo de esta contribución es analizar la evolución de la producción científica en este tema, considerando las publicaciones realizadas en revistas nacionales e internacionales. Se cuantificaron aspectos como la cantidad de trabajos publicados por año, cantidad de autores por publicación, países de publicación y temas considerados en cada trabajo. Se observa un incremento en la cantidad de publicaciones a partir de 1990, registrándose el máximo en el año 2003, que estaría relacionado al aumento de investigadores dedicados al tema en nuestro país. Aumenta también el número de autores (tres a seis) a partir de ese año, lo cual coincide con análisis bibliométricos previos enfocados en otras áreas paleontológicas. El país donde más trabajos se han publicado es Argentina (58,5\%), aumentando las publicaciones en otros países a partir de 1990 , debido a la globalización del conocimiento y cooperación con especialistas extranjeros. La bioestratigrafía $(68,6 \%)$ y la taxonomía $(24,0 \%)$ son los principales temas desarrollados, debido a que la primera ha adquirido interés a nivel mundial y la segunda constituye la base de las otras líneas de investigación. Otros aspectos paleobiológicos se han beneficiado durante los últimos años con el uso de métodos e índices estadísticos que otorgan soportes confiables.

*Proyecto subsidiado por ANPCYT, PICT 2016-2019-0558 de la Agencia Nacional de Promoción Científica y Tecnológica. 


\title{
CONTENIDO PALEONTOLÓGICO DE LOS NIVELES BASALES DE LA FORMACIÓN LA CHILCA, EN LA QUEBRADA POBLETE NORTE, TALACASTO (PRECORDILLERA CENTRAL), SAN JUAN
}

\author{
F.E. LóPEZ ${ }^{1}$, L.I. LeÓN ${ }^{1}$, C. KAufMann ${ }^{2}$ Y V.H. CONTRERAS ${ }^{1}$ \\ ${ }^{1}$ Instituto de Geología Dr. Emiliano P. Aparicio y Departamento de Geología, Facultad de Ciencias Exactas, Físicas y Naturales, Universidad \\ Nacional de San Juan. Av. Ignacio de la Roza 590 (0), J5402DCS Rivadavia, San Juan, Argentina. fernandohenrylopez@gmail.com; \\ lauleon@unsj-cuim.edu.ar; vcontre@unsj-cuim.edu.ar \\ ${ }^{2}$ Consejo Nacional de Investigaciones Científicas y Tecnológicas (CONICET). Instituto de Investigaciones Mineras, Facultad de Ingeniería, Uni- \\ versidad Nacional de San Juan. Av. Libertador Gral. San Martín 1109, J5400ARL San Juan, San Juan, Argentina. cintiakaufmann@gmail.com
}

Los niveles fosilíferos de la Formación La Chilca han sido ampliamente estudiados en el ámbito de la Precordillera Central, prestando especial atención a las localidades de: Quebrada Ancha, Baños de Talacasto y Cerro del Fuerte. En la presente contribución se da a conocer un nuevo sitio de exposición de esta unidad, ubicada en la Quebrada Poblete Norte (31 ${ }^{\circ} 00^{\prime}$ $01,16^{\prime \prime}$ S; $68^{\circ} 46^{\prime} 25,92^{\prime \prime}$ W), en el sector austral de la sierra de Talacasto (departamento Ullum), la cual hasta el momento no presentaba descripciones de su contenido paleontológico. En este sector, la unidad se caracteriza por un típico arreglo grano-estrato creciente, coincidente con lo descripto por diversos autores en secciones cercanas. En sus niveles basales se han recuperado las primeras evidencias fosilíferas de la zona, distribuidas en cinco niveles. Se identifican graptolitos en regular a buen estado de conservación, los que en forma preliminar se asignan a: Climacograptus sp., Normalograptus sp., Lagarograptus praecinaces? Cuerda, Rickards y Cingolani, y Monograptus priodon? Bronn. Además, se reconocen braquiópodos con ornamentación simple. La asociación de la graptofauna reconocida se correspondería con la hallada en los Baños de Talacasto y el Cerro del Fuerte, indicando una edad Hirnantiana a Rhudaniana.

\section{HALLAZGO DE RESTOS DE PROTEROTHERIIDAE (MAMMALIA, LITOPTERNA) EN EL PLIOCENO TEMPRANO DEL VALLE CALCHAQUÍ (SALTA, ARGENTINA)}

\author{
G.M. López' ${ }^{1}$ C.I. Galli², D.A. CROFT ${ }^{3}$ y M.A. Reguero ${ }^{1}$
}

1División Paleontología de Vertebrados, Museo de La Plata, Facultad de Ciencias Naturales y Museo. Universidad Nacional de La Plata. Paseo del Bosque s/n, B1900FWA La Plata, Buenos Aires, Argentina.glopez@fcnym.unlp.edu.ar; regui@fcnym.unlp.edu.ar

${ }^{2}$ Facultad de Ingeniería, Universidad Nacional de Salta. Av. Doctor Bernardo Houssay 1099, 4400 Salta, Salta, Argentina. claudiagalli@fibertel.com.ar

32Department of Anatomy, Case Western Reserve University. 10900 Euclid Ave., 44106 Cleveland, Ohio, Estados Unidos.dcroft@case.edu

En el Valle Calchaquí (Salta, Argentina), las acumulaciones de sedimentos clásticos continentales del Cenozoico tienen muy buena exposición y constituyen el Grupo Payogastilla. Estos depósitos se acumularon desde el Eoceno Medio a Plioceno Tardío en una cuenca de antepaís, fragmentada como consecuencia del levantamiento Andino. La Formación San Felipe, de 600 m de potencia, representa la última etapa en la evolución de esta cuenca y está caracterizada por facies de un sistema de abanico fluvial entrelazado, que progresivamente cambia a un sistema fluvial entrelazado gravoso. Inmediatamente por encima del contacto con la infrayacente Formación Palo Pintado, se encuentra una toba que en base a dataciones U/Pb arrojó una edad de 5,27 £ 0,28 Ma. Dos metros por arriba de este nivel tobaceo se recolectó un fragmento maxilar derecho con P2-P4 asociado a restos postcraneanos, representados por un fragmento proximal de húmero, los extremos proximal y distal del metacarpo III y 8 cuerpos vertebrales mal preservados. La morfología de estos restos permite referirlos a los Litopterna Proteotheriidae. Los premolares presentan coronas protohipsodontas, cíngulo anterolingual evidente e hipocono bien desarrollado en P3-P4, que le confieren a estos dientes un contorno cuadrangular. Por las características mencionadas y por su tamaño, estos restos pueden referirse tentativamente a Epitherium Ameghino. La distribución estratigráfica de este género (i.e., Huayqueriense-Montehermosense) es congruente con la antigüedad de los niveles portadores de la Formación San Felipe y de confirmarse la asignación a este género se ampliaría a su distribución geográfica a la provincia de Salta. 


\title{
MORPHOLOGICAL VARIABILITY IN THE GASTROPOD PERISSODONTA MARTENS (= STRUTHIO- LARELLA) FROM THE EARLY MIOCENE OF TIERRA DEL FUEGO
}

\author{
M.I. Lopez CABrera ${ }^{1}$ y E.B. Olivero ${ }^{1 *}$
}

${ }^{1}$ Laboratorio de Geología Andina. Centro Austral de Investigaciones Científicas (CADIC), Consejo Nacional de Investigaciones Científicas y Tecnológicas (CONICET). B. Houssay 200, V9410CAB Ushuaia, Tierra del Fuego, Argentina. mariaolivero22@hotmail.com; emolivero@gmail.com

High morphological variability in Neogene struthiolarid gastropods from Patagonia and Tierra del Fuego has been recognized since earliest studies by Ihering, Steinmann, Wilckens and Ortmann. In particular, when Steinmann and Wilckens erected the genus Struthiolarella, which is now interpreted as a junior synonym of Perissodonta, they remark the difficulty of differentiating S. ameghinoi (Ihering) — the type species of Struthiolarella - from other species or varieties recognized in the Cenozoic of Patagonia, Chile, and Tierra del Fuego. Nonetheless, in Tierra del Fuego four species/subspecies of the struthiolarid gastropod Perissodonta have been recognized, including: Struthiolaria chilensis fueguina Ihering, Struthiolarella fueguina (Ihering), Struthiolarella ameghinoi (Ihering), and Struthiolarella densestriata (Ihering). These species/subspecies of Struthiolarella were mainly differentiated on the basis of sculptural features of the shell, including differences in the axial and spiral ornamentation, density and number of fine spiral threads and strong spiral cords in the body whorl, and axial tubercles or nodes on the periphery of the whorl. A preliminary study of more than 100 specimens referred to Perissodonta from the classical localities of the Sierra de Carmen Silva and new localities including Punta Basílica, Cabo Viamonte, and Río Irigoyen indicates that all these specimens have slightly globose to fusiform medium-size shell, with thick inductura. However, axial and spiral ornamentation of the shell vary markedly between specimens, supporting the conclusions of earlier researchers. Accordingly, we interpret that the studied material corresponds to a single highly variable species that can be accommodate in Perissodonta ameghinoi (lhering), of probably Early Miocene age.

*Financial suport by PIDUNT dF-A1.

\section{HELECHOS FÓSILES DE LA FORMACIÓN HUITRERA (EOCENO) EN LA LOCALIDAD ARROYO CHACAY, PROVINCIA DE RÍO NEGRO, ARGENTINA}

\author{
M.A. Machado ${ }^{1}$, E.I. Vera ${ }^{1}$ Y M. PASSALIa ${ }^{2}$
}

\begin{abstract}
'Museo Argentino de Ciencias Naturales "Bernardino Rivadavia" (MACN)-Consejo Nacional de Investigaciones Científicas y Tecnológicas (CONICET). Av. Ángel Gallardo 470, C1405DJR Ciudad Autónoma de Buenos Aires, Argentina.agus.machado7@gmail.com; evera@macn.gov.ar ${ }^{2}$ Instituto de Investigaciones en Biodiversidad y Medioambiente (INIBIOMA), Consejo Nacional de Investigaciones Científicas y TecnológicasUniversidad Nacional del Comahue (CONICET-UNCO). Av. de los Pioneros 2350, R8402ALP San Carlos de Bariloche, Río Negro, Argentina. passaliam@gmail.com
\end{abstract}

En esta contribución se da a conocer preliminarmente el contenido pteridofítico de la Formación Huitrera en la localidad Arroyo Chacay (provincia de Río Negro, Argentina). Los especímenes estudiados presentan un muy buen grado de preservación e incluyen restos de frondes fértiles y estériles. Dentro de los materiales estudiados se reconocen abundantes restos de Todea amissa Carvalho (Osmundaceae), dos especies diferentes de Cyclosorus (Thelypteridaceae), y restos fértiles comparables a Dicksonia patagonica Berry (Dicksoniaceae?). Se reconocen además en la asociación restos de posibles Hymenophyllaceae y Blechnaceae, junto con otros taxones de afinidades dudosas. Esta contribución representa el primer estudio del contenido florístico de la unidad en esta localidad, y permite incrementar el conocimiento sobre las paleofloras del Eoceno de Patagonia. 


\title{
AVANCES EN EL ESTUDIO TAXONÓMICO Y FILOGENÉTICO DE DOLICHOTINAE (RODENTIA, CAVIIDAE) DEL MIOCENO TARDÍO-PLIOCENO
}

\author{
M.C. Madozzo JaÉn ${ }^{1}$ Y M.E. PÉrez ${ }^{1 *}$ \\ ${ }^{1}$ Consejo Nacional de Investigaciones Científicas y Tecnológicas (CONICET). Museo Paleontológico Egidio Feruglio. Av. Fontana 140, U9100GYO \\ Trelew, Chubut, Argentina.cmadozzojaen@mef.org.ar; mperez@mef.org.ar
}

La subfamilia Dolichotinae está representada en la actualidad por Dolichotis patagonum y D. salinicola, pero en el registro fósil su diversidad es mayor. Históricamente, se definieron 15 especies fósiles distribuidas en cinco géneros: Orthomyctera, Propediolagus, Pliodolichotis, Prodolichotis, Dolichotis. La mayoría de dichos taxa presenta historias taxonómicas complejas que deben ser clarificadas. En las últimas décadas el conocimiento de la taxonomía, la filogenia e historia evolutiva de Caviidae se ha incrementado, principalmente en lo que respecta a las formas más basales de la familia y a los clados derivados Hydrochoerinae y Caviinae. Sin embargo, los Dolichotinae son hasta el momento pobremente conocidos. Avances en el estudio de la taxonomía y filogenia de taxones fósiles de dolicotinos indican una reducción de la diversidad genérica en las formas del Mioceno Tardío-Plioceno. Resultados preliminares indicarían una estrecha relación de Orthomyctera, tradicionalmente considerado Dolichotinae, con la subfamilia Caviinae. Si bien la diversidad de Dolichotinae en el pasado es posiblemente menor a la originalmente descripta, sigue siendo más abundante que en la actualidad. La reciente inclusión de Prodolichotis prisca en análisis filogenéticos corroboran su validez y su posición basal respecto a Dolichotis. El estudio de la taxonomía alfa de formas fósiles y su inclusión en análisis filogenéticos, aumenta el conocimiento de la diversidad de Dolichotinae y completa los patrones de distribución. No obstante, restan estudios de numerosas formas fósiles de Dolichotinae registradas en sedimentos del Mio-Plioceno de las distintas regiones de Argentina (noroeste, noreste y región pampeana).

*Proyecto financiado por PICT -201-0566.

\section{PUESTA EN VALOR Y DIGITALIZACIÓN DE LA COLECCIÓN SAEMANN}

\author{
L.S. Marin ${ }^{1}$, M.A. Tanuz ${ }^{2}$ y B. Aguirre-Urreta ${ }^{3}$
}

${ }^{1}$ Facultad de Ciencias Exactas y Naturales, Universidad de Buenos Aires. Intendente Güiraldes 2160, Ciudad Universitaria C1428EGA Ciudad Autónoma de Buenos Aires, Argentina. Iucianasabina_marin@hotmail.com

2Departamento de Geología, Facultad de Ciencias Exactas y Naturales, Universidad de Buenos Aires. Intendente Güiraldes 2160, Ciudad Universitaria, C1428EGA Ciudad Autónoma de Buenos Aires, Argentina.mtanuz@gl.fcen.uba.ar

${ }^{3}$ Departamento de Geología, Facultad de Ciencias Exactas y Naturales, Instituto de Estudios Andinos "Don Pablo Groeber" (IDEAN), Universidad de Buenos Aires-Consejo Nacional de Investigaciones Científicas y Tecnológicas (CONICET). Intendente Güiraldes 2160, Ciudad Universitaria - Pabellón II, C1428EGA Ciudad Autónoma de Buenos Aires, Argentina. aguirre@gl.fcen.uba.ar

En el año 1865, Pellegrino Strobel, primer profesor de Geología de la Universidad de Buenos Aires, solicitó la compra en París de la primera colección de material paleontológico y geológico de la Facultad, con el objetivo de usarla como material didáctico en clase. Dicha colección, Ilamada Saemann, fue adquirida por 1510 francos al coleccionista Louis Saemann, quien durante muchos años trabajó en la famosa Casa Krantz, en Berlín, antes de abrir su tienda en París. La colección estaba compuesta por más de 400 piezas fósiles de invertebrados, plantas y vertebrados, ordenados estratigráficamente, además de minerales y rocas, provenientes de los yacimientos más conocidos de la época, como el Cámbrico de la Montage Noir y de Bohemia, el Ordovícico de Devonshire, el Silúrico de Gotland y Dudley, el Devónico de Eiffel y Geroistein y el Carbonífero de Sarthe, entre otros. Se destaca que la adquisición de esta colección ubicó a la Universidad de Buenos Aires entre las grandes casas de estudio que disponían de este tipo de materiales en esa época. Debido a la importancia histórica de la Colección Saemann, se realizaron tareas de puesta en valor y digitalización, que incluyeron la centralización de los materiales recuperados (alrededor de 350 piezas), la preservación y fotografiado de los ejemplares, el escaneado de etiquetas originales y la recopilación de la información obtenida de las etiquetas y de los catálogos en una planilla digital. El trabajo realizado permitirá a futuro el acceso virtual a la colección para todas las personas que así lo requieran. 


\title{
PALINOMORFOS NO POLÍNIICOS EN MATAS MICROBIANAS MODERNAS EN UN AMBIENTE COSTERO MARGINAL (BAHÍA SAN BLAS, ARGENTINA)
}

\author{
M.A. Martínez ${ }^{1}$, D.G. Cuadrado², M.V. Bianchinotti ${ }^{3}$ y G.R. Guerstein ${ }^{1 *}$ \\ ${ }^{1}$ Instituto Geológico del Sur (INGEOSUR)-Consejo Nacional de Investigaciones Científicas y Tecnológicas (CONICET)-Departamento de Geolo- \\ gía, Universidad Nacional del Sur. Av. Alem 1253, B8000CPB Bahía Blanca, Buenos Aires, Argentina. martinez@criba.edu.ar; \\ raquel.guerstein@uns.edu.ar \\ 2Instituto Argentino de Oceanografía (IADO), Consejo Nacional de Investigaciones Científicas y Técnicas (CONCIET)-Departamento de Geolo- \\ gía, Universidad Nacional del Sur. Florida 500, B8003JSD Bahía Blanca, Buenos Aires, Argentina. cuadrado@criba.edu.ar \\ ${ }^{3}$ Centro de Recursos Naturales Renovables de la Zona Semiárida (CERZOS), Consejo Nacional de Investigaciones Científicas y Técnicas \\ (CONICET)-Departamento de Biología, Bioquímica y Farmacia, Universidad Nacional del Sur. San Juan 670, B8000ICN Bahía Blanca, Buenos \\ Aires, Argentina. vbianchi@uns.edu.ar
}

El estudio palinológico de testigos obtenidos en matas microbianas desarrolladas en un ambiente marino-marginal moderno permite determinar patrones de palinomorfos no polínicos (PNP) y palinofacies como una herramienta para reconocer este tipo de ambientes en secciones fósiles. Los depósitos analizados en Paso Seco, Bahía San Blas, provincia de Buenos Aires $\left(40^{\circ} 33^{\prime} \mathrm{S} ; 62^{\circ} 14^{\prime} \mathrm{O}\right)$, corresponden a un evento progradante representado por un canal de marea antiguo que se inunda frecuentemente con la marea. Esta planicie expuesta se caracteriza por la presencia de matas microbianas (MM) superficiales, de ca. $2 \mathrm{~cm}$ espesor, producidas por la actividad de cianobacterias y diatomeas bentónicas que colonizan los sedimentos. La actividad microbiana da lugar a un entretejido de filamentos y sustancias poliméricas extracelulares (EPS) que atrapan y aglutinan sedimentos, esporomorfos, PNP y materia orgánica (MO) estructurada y amorfa (MOA). En profundidad se identifican MM antiguas, produciendo la bioestabilización del sedimento y ello se traduce en un alto potencial de preservación de eventos sedimentológicos. En las palinofacies de la superficie óxica, el hábitat propicio para la proliferación de cianobacterias, se identificaron gran cantidad de filamentos junto con restos fúngicos de pared melanizada de composición quitinosa. Por otra parte, las palinofacies de las MM infrayacentes están dominadas por fitoclastos, MOA, esporomorfos, PNP y abundante pirita framboidal, en excelente estado de preservación, posiblemente vinculadas a la preservación generada por EPS en un medio más reductor. Entre los PNP predominan restos fúngicos, quistes de dinoflagelados (principalmente Operculodinium, Polysphaeridium y escasos Spiniferites), palinoforaminíferos y remanentes de algas dulceacuícolas.

*Proyecto financiado por PIP-CONICET N¹282 y PGI SECyT -UNS N²4/H138 y N²4/H142.

\section{UN NUEVO TOXODONTIA (MAMMALIA, NOTOUNGULATA) DEL PALEÓGENO DE LA PATAGONIA ARGENTINA}

\section{G. Martínez ${ }^{1}$, M.T. Dozo ${ }^{2}$ y M.R. CianCio $3 *$}

${ }^{1}$ Museo de Paleontología de la Facultad de Ciencias Exactas Físicas y Naturales,Universidad Nacional de Córdoba. Av. Vélez Sársfield 249, X5000JJC Córdoba, Córdoba, Argentina. gmartinezpsf@gmail.com

${ }^{2}$ Instituto Patagónico de Geología y Paleontología (IPGP), Centro Nacional Patagónico (CENPAT)-Consejo Nacional de Investigaciones Científicas y Tecnológicas (CONICET). Boulevard Brown 2915, U9120ACD Puerto Madryn, Chubut, Argentina. dozo@cenpat-conicet.gob.ar ${ }^{3}$ División Paleontología de Vertebrados, Museo de La Plata, Facultad de Ciencias Naturales y Museo, Universidad Nacional de La Plata. Paseo del Bosque s/n, B1900FWA, La Plata, Buenos Aires, Argentina. mciancio@fcnym.unlp.edu.ar

Dentro del suborden Toxodontia (Mammalia, Notoungulata) existen agrupamientos cuya monofilia está ampliamente consensuada (Homalodotheriidae, Leontiniidae y Toxodontidae) y numerosos taxones cuyo patrón de agrupamiento no parece ser consistente con hipótesis previas de ancestralidad (no constituyen clados). El espécimen que aquí se presenta (MPEF PV 2578) corresponde a la región anterior de un cráneo y mandíbula asociados de un individuo adulto con la dentición casi completa (desgaste medio-avanzado), el cual se muestra estrechamente relacionado a taxones asignados originalmente a "Notohippidae", uno de los agrupamientos sin respaldo en hipótesis filogenéticas más actuales. Algunas características como la fuerte retracción de los procesos ascendentes de los premaxilares, un arco premaxilar amplio y redondeado combinado con una fuerte constricción palatal y una particular asociación de características de la morfología oclusal, lo diferencian de formas afines como Eomorphippus, Rhynchippus, Eurygenium o Pascualihippus, por lo que es considerado tentativamente como un nuevo taxón. Fue hallado en Barrancas Blancas (SE de Chubut, Argentina) en niveles correspondientes a la sección media de la Formación Sarmiento. La fauna de esta localidad (mayormente restos fragmentarios) incluye además xenartros, litopternos y otros notoungulados y su composición sugiere una asociación faunística postinguiririquense-predeseadense (¿Oligoceno medio?). Más allá del valor intrínseco de un probable nuevo taxón y de ser el resto más completo proveniente de esta localidad, MPEF PV 2578 resulta particularmente relevante puesto que se ubica en una región controvertida del cladograma (aquella que involucra a los parafiléticos "Notohippidae"), promoviendo la discusión de la sistemática de un grupo complejo como el de los Toxodontia. 
*Proyecto financiado por 2015-2017. CONICET. Mamíferos y paleoambientes del Oligoceno y Mioceno del Este del Chubut (Patagonia central): localidades clave para entender la historia evolutiva de los mamíferos fósiles de América del Sur. PIP Nº 11220150100113 (Res. N 0111/16, 20/01/16). Director: María T eresa Dozo

\title{
EVOLUCIÓN MORFOLÓGICA DEL GÉNERO STEINMANELLA CRICKMAY (BIVALVIA) EN LA FOR- MACIÓN AGRIO, CRETÁCICO INFERIOR DE LA CUENCA NEUQUINA
}

\begin{abstract}
P.S. Milla Carmona ${ }^{1}$, D.G. LaZO ${ }^{1}$ y I.M. Soto ${ }^{2 *}$
'Instituto de Estudios Andinos "Don Pablo Groeber" (IDEAN), Universidad de Buenos Aires-Consejo Nacional de Investigaciones Científicas y Tecnológicas (CONICET). Intendente Güiraldes 2160, Ciudad Universitaria - Pabellón II, C1428EGA Ciudad Autónoma de Buenos Aires, Argentina.millacarmona@gl.fcen.uba.ar; dlazo@gl.fcen.uba.ar

${ }^{2}$ Instituto de Ecología, Genética y Evolución de Buenos Aires (IGEBA), Universidad de Buenos Aires-Consejo Nacional de Investigaciones Científicas y Tecnológicas (CONCIET). Intendente Güiraldes 2160, C1428EGA Ciudad Autónoma de Buenos Aires, Argentina. soto@ege.fcen.uba.ar

Steinmanella (Trigonioida, Myophorelloidea) es uno de los géneros de bivalvos fósiles más comunes y conspicuos del Cretácico Inferior de Cuenca Neuquina, registrándose en numerosos niveles estratigráficos a lo largo del intervalo Valanginiano inferior-Hauteriviano superior. El objetivo del presente trabajo es la caracterización de los patrones de evolución que exhibe Steinmanella en su morfología externa a lo largo del intervalo temporal representado por la Formación Agrio (Valanginiano tardío-Hauteriviano tardío). Para ello, se seleccionaron 97 especímenes en buen estado de preservación, pertenecientes a las especies, S. pehuenmapuensis Leanza, S. transitoria Steinmann y S. vacaensis Weaver, registradas en 11 de las 13 biozonas/subzonas de amonoideos reconocidas para la unidad. El tamaño y geometría de la conchilla fueron cuantificados usando morfometría geométrica en tres dimensiones mediante la utilización de un escáner manual 3D, mientras que la ornamentación fue cuantificada con medidas morfométricas tradicionales (conteos de costillas y tubérculos). El cambio a través del tiempo de cada una de estas variables fue estudiado utilizando análisis de series temporales. Los resultados muestran que tanto la ornamentación como el tamaño de Steinmanella cambian siguiendo un modelo de evolución al azar, producido por la interacción entre los patrones de cambio anagenético de cada especie y el cambio asociado a los eventos de especiación. Por otro lado, la geometría de la conchilla presenta un cambio sostenido en el tiempo desde configuraciones aproximadamente triangulares, delgadas y con carena marginal bien definida, hacia formas aproximadamente rectangulares, más globosas y con carena marginal atenuada.
\end{abstract}

*Proyecto financiado por subsidios APA para estudiantes de postgrado (P.S. M.C.), ANPCyT PICT 2015-1381 (D.G.L.).

\section{TAXONOMIC AND BIOSTRATIGRAPHIC ANALYSIS OF THE ORDOVICIAN GRAPTOLITES FROM LA CIÉNAGA DE PURMAMARCA, JUJUY PROVINCE, ARGENTINA}

\author{
J.M. Navarro"1, B.A. Toro², D.F. Muñoz², And N.C. Herrera SÁnChez ${ }^{2 *}$ \\ ${ }^{1}$ Facultad de Ciencias Exactas, Físicas y Naturales, Universidad Nacional de Córdoba. Av. Vélez Sársfield 1611, X5016CGA Córdoba, Córdoba, \\ Argentina.matiasnavarro93@gmail.com \\ ${ }^{2}$ Centro de investigaciones en Ciencias de la Tierra (CICTERRA), Consejo Nacional de Investigaciones Científicas y Técnicas (CONICET) - Facultad \\ de Ciencias Exactas, Físicas y Naturales, Universidad Nacional de Córdoba. Av. Vélez Sársfield 1611, X5016CGA Córdoba, Córdoba, Argentina. \\ btorogr@mendoza-conicet.gov.ar;.df.munoz@conicet.gov.ar; nexxysherrera@gmail.com
}

The detailed paleontological sampling of the Acoite Formation, carried out at La Ciénaga de Purmamarca area, allowed to obtain a variety of graptolites among which we can highlight Kiaerograptus kiaeri Monsen, recorded for the first time in this section. The taxonomic study confirmed the occurrence of Paratetragraptus approximatus Nicholson in the lower part of the Acoite Formation, and permitted the identification of numerous specimens of Cymatograptus rigoletto Maletz, Rushton and Lindholm. Baltograptus vacillans Tullberg and Baltograptus geometricus Törnquist were recorded at the intermediate levels, while Baltograptus cf. B. turgidus Lee and Baltograptus kunmingensis Ni at the upper levels of the mentioned unit. The biostratigraphic analysis indicates that the Tetragraptus phyllograptoides? (FI 1), Tetragraptus akzharensis (FI 1) and the lower part of Baltograptus cf. B. deflexus (FI2) Biozones are present in La Ciénaga de Purmamarca, documenting the early Floian to early middle Floian age of the studied deposits. The species K. kiaeri, corresponding to the Aorograptus victoriae Biozone, indicates a late Tremadocian age for the fossil-bearing levels, which are considered a section of the Santa Rosita Formation. Additionally, the studied stratigraphic interval is regionally correlated with the lower to middle part of the Acoite Formation of Los Colorados, Sierra de Aguilar and Santa Victoria, in Argentina; and with equivalent levels exposed in Cieneguillas and Chaupi Uno, in Bolivia. It is also globally correlated with Baltoescandinavia, Avalonia and Morocco, confirming the paleobiogeographic affinities of the Central Andean Basin with other regions of intermediate to high latitudes. 
*Financial support by Agencia Nacional de Promoción Científica y Tecnológica (ANPCyT-FONCyT) PICT 2016-0558. T his is a contribution to the IUGS-IGCP project 653 'The onset of the Great Ordovician Biodiversification Event'.

\title{
UN POSIBLE CASO DE ROOT SUCKERING EN UNA CUPRESSACEAE S.L. DEL CRETÁCICO INFERIOR DE PATAGONIA
}

\author{
C.I. NUnes' ${ }^{1}$, J. BODNAR ${ }^{2}$, I.H. ESCAPA ${ }^{1}$ y N.R. CÚNEO ${ }^{1 *}$
}

1'Museo Paleontológico Egidio Feruglio, Av. Fontana 140, U9100GYO Trelew, Chubut, Argentina.cnunes@mef.org.ar; iescapa@mef.org.ar; rcuneo@mef.org.ar

2División de Paleobotánica, Museo de La Plata, Facultad de Ciencias Naturales y Museo, Universidad Nacional de La Plata. Paseo del Bosque s/n, B1900FWA La Plata, Buenos Aires, Argentina. jbodnar@fcnym.edu.ar

La capacidad de producir troncos secundarios a partir de retoños de la raíz, o root suckering, es un mecanismo poco usual en las coníferas, presente en algunas especies actuales de Araucariaceae, Podocarpaceae, y Cupressaceae. El brote mediante root suckers puede ocurrir como respuesta a estrés ambiental, o como parte normal del desarrollo en especies clonales. Aunque es un mecanismo ecológicamente relevante en términos de supervivencia, regeneración y propagación, sus aspectos evolutivos han sido poco explorados en el registro fósil. En la Formación Cerro Barcino (localidad de "La Flecha", provincia del Chubut), de edad albiana tardía (101 Ma), se hallaron dos troncos permineralizados, conectados a través de la raíz, separados por una distancia de $90 \mathrm{~cm}$. Dichos restos fueron estudiados anatómicamente y asignados a un nuevo género de afinidad cupresácea. Ambos especímenes poseen un diámetro de 25 cm y son ortótropos. La raíz es horizontal, y tiene un diámetro de $15 \mathrm{~cm}$. Macroscópicamente, se observan en el leño de la raíz zonas formando patrón en espiral, que correspondería a brotes o ramificaciones. Anatómicamente, el xilema secundario de la raíz y los troncos difieren en caracteres cuantitativos, siendo en la raíz más frecuentes las punteaduras de las traqueidas biseriadas y menor el número de punteaduras por campo de cruzamiento. La morfología general y la arquitectura de los especímenes permiten inferir que esta planta crecía clonalmente a través de root suckers, indicando que se trata de un mecanismo que, al menos en las cupresáceas, está presente de hace al menos 100 Ma.

*Proyecto financiado por ANPCyT PICT 2014-2433 (NRC), EVC-CIN EVC-UNPSJB3805 (CIN).

\section{SYSTEMATICS AND BIOSTRATIGRAPHY OF SANTONIAN-LOWER CAMPANIAN SCAPHITIDAE (AMMONOIDEA, ANCYLOCERATACEA) FROM ANTARCTICA}

\author{
E.B. OLIVERO ${ }^{1}$ Y M.E. RAFFI ${ }^{1 *}$
}

${ }^{1}$ Laboratorio de Geología Andina, Centro Austral de Investigaciones Científicas (CADIC)-Consejo Nacional de Investigaicones Científicas y Tecnológicas (CONICET). B. Houssay 200, V9410CAB Ushuaia, Tierra del Fuego, Argentina.emolivero@gmail.com; eugeniaraffi@gmail.com

Lack of recognition of sexual dimorphism in scaphitid ammonites was always a source of great taxonomic confusion, particularly in the Ottoscaphitinae where the macroconchs of Yezoites Yabe are quite similar to the typical Scaphitinae genus Hoploscaphites Nowak. In Antarctica, the Santonian-early Campanian record of Hoploscaphites is a major, not resolved problem because the genus is restricted worldwide to the late Campanian-Maastrichtian. Here, we demonstrated that all previous mentions of Hoploscaphites in Antarctica are in error and that they all consist of macroconchs of at least four species of Yezoites. Antarctic scaphitids are abundant in the Alpha Member, Santa Marta Formation, with more than 100 specimens of tiny Yezoites recovered from Santonian-early Campanian strata. The record includes in succession: a) macroconchs and microconchs of at least two species of Yezoites, Santonian. One species, previously classified as Yezoites sp. 1 and Hoploscaphites? sp. 1, is quite similar and probably co-specific with Yezoites australis Kennedy and Klinger, from South Africa, the other, Yezoites sp. 2, is probably a new species; and b), two additional species of Yezoites previously referred to Yezoites sp. 3, Yezoites sp. 4 and Hoploscaphites? sp. 2, both including micro- and macroconchs, Santonian-early Campanian. On the basis of similar stratigraphic distribution and shell features, it is concluded that previous records of Santonian-early Campanian Hoploscaphites in Antarctica are in error and that the material is best classified as the macroconch of different Yezoites species.

*Proyecto financiado por PIDUNT dF-A1. 


\title{
EL RESCATE DE UN NESODON EN COMALLO (RÍO NEGRO) Y SU PUESTA EN VALOR POR PARTE DE LA ASOCIACIÓN PALEONTOLÓGICA BARILOCHE
}

\author{
M.A. Pagliaro1 ${ }^{1}$, B. Villarroel ${ }^{1}$, K. Ulloa ${ }^{1}$, R. Gallardo ${ }^{1}$, D.M. Katzenell ${ }^{1}$, A. Iglesias ${ }^{1}$ y A. Paulina Carabajal ${ }^{1}$ \\ ${ }^{1}$ Museo Paleontológico Bariloche. 12 de Octubre y Sarmiento, R8400 San Carlos de Bariloche, Río Negro, Argentina. \\ miguelangel.pagliaro@gmail.com; villarroelbelen92@gmail.com; ulloaka@gmail.com; rociogallardo2009@live.com.ar; davidhundenell@gmail.com; \\ ari_iglesias@yahoo.com.ar; premjisaurus@yahoo.com.ar
}

¿Cómo es que un grupo de elementos ordenados de una forma determinada pasan a ser valiosos de un momento al otro? Son los fósiles, materiales que parecen estar esperando para ser descubiertos. Entonces alguien los encuentra, de forma ocasional o consecuente, y en aquel momento toman un valor. Son rescatados, pero ¿por qué? ¿para qué? ¿para quienes? ¿se rescatan para formar parte de una lista, para ser protegidos, para investigarse, para mostrarse, para guardarse en una colección?. En este ejemplo, presentamos el rescate y preparación de un esqueleto de Nesodon realizado por la Asociación Paleontológica de Bariloche (APB) en ocasión de un hallazgo casual por una empresa en Comallo, con participación de socios coordinados por investigadores de la APB. El voluntariado se realizó en todas las etapas a lo largo de 3 años (hallazgo, extracción, preparación, puesta en valor). El objetivo de hacer partícipes a socios aficionados, a la Secretaría de Cultura y a la empresa constructora, fue incluirlos como parte de la comunidad logrando que se apropien de la importancia del patrimonio, involucrándose en su protección. Como resultado, esta participación incentivó y generó un compromiso por parte de la comunidad hacia las piezas patrimoniales, al tiempo que estableció las primeras experiencias y herramientas de formación técnica en tareas afines al museo (preparación, confección de camas de yeso, soportes y moldes) y la investigación (anatomía). El cráneo y el más completo miembro anterior de Nesodon están listos para su estudio y exposición al público en Bariloche.

\section{ANIMATRÓNICA PARA LA DIVULGACIÓN CIENTÍFICA DE LA PALEONTOLOGÍA}

\author{
H.N. Pailos ${ }^{1}$, G.A. Montes ${ }^{1}$, A.A. Tauber ${ }^{2}$ y S. Gonzalez ${ }^{1 *}$ \\ ${ }^{1}$ Laboratorio de Animatrónica y Control Dinámico, Universidad Nacional de Córdoba. Velez Sársfield 1611, X5016CGA Córdoba, Córdoba, \\ Argentina.hpailos@gmail.com; abelgermanmontes@gmail.com; samuelluisgonzalez26@gmail.com \\ 2Museo de Ciencias Naturales "Arturo Umberto Illia". Av. Leopoldo Lugones 392, X5000HZQ Córdoba, Córdoba, Argentina. \\ adantauber@gmail.com
}

El objetivo del Laboratorio es diseñar y construir robots que representen especies extintas con el fin de ser exhibidas en museos, centros de interpretación y parques. Para construir un animatrónico cuya morfología sea verosímil a un animal extinto, debemos intersectar varias disciplinas. Una vez elegida la especie a robotizar, se consultan a biólogos y paleontólogos que facilitan la información del registro fósil; en el siguiente paso se hace el estudio biomecánico básico cuyo resultados son los grados de movilidad y de libertad de cada articulación; luego se propone un sistema mecánico que emule los movimientos para que estos sean realistas; este modelo debe ser aprobado por los paleontólogos para realizar la última etapa que es la realización física. El animatrónico queda construido con las piezas mecánicas y los motores que moverán cada parte del robot. El movimiento es programado en un procesador digital que es el cerebro del robot. La piel es el toque final para que el robot pueda ser presentado al público en general. Esta tarea conlleva en trabajo mancomunado de paleontólogos, biólogos, mecánicos, ingenieros, paleoartistas y programadores. Algunos de los proyectos realizados son: Abelisaurus (primer dinosaurio robot argentino), Saltasaurus (cuello robotizado), Tapejara (primer pterosaurio robotizado de Brasil), pteranodones, Nigersaurus, Jeholopterus, Liopleurodon y gliptodontes.

*Proyecto financiado por Universidad Nacional de Córdoba. 


\title{
ABORDAJE DE LA ANALOGÍA COMO RECURSO EN LA ENSEÑANZA DE LAS CIENCIAS DESDE UN ENFOQUE EPISTEMOLÓGICO: EL CASO DE LA PALEONTOLOGÍA
}

\author{
E.E. Palópolo ${ }^{1}$ y M.A. Diez ${ }^{2 *}$ \\ ${ }^{1}$ Instituto de Investigación en Paleobiología y Geología (IIPG)-Consejo Nacional de Investigaciones Científicas y Tecnológicas (CONICET), Uni- \\ versidad Nacional de Río Negro. Av. Roca 1242, R8332EXZ Gral. Roca, Río Negro, Argentina. eepalopolo@unrn.edu.ar \\ Escuela de Geología, Paleontología y Enseñanza de la Ciencias (GEPEC), Sede Alto Valle y Valle Medio, Universidad Nacional de Río Negro. \\ Estados Unidos 750, R8332BRJ, General Roca, Río Negro, Argentina.mdiez@unrn.edu.ar
}

La analogía como recurso en la enseñanza de las ciencias suele pasar desapercibida para los docentes y científicos. Sin embargo, las competencias cognitivas y el aprendizaje funcionan como procesos cerebrales analógicos. Cuando una persona aborda un fenómeno o acontecimiento que excede su conocimiento, recurre a saberes previos en torno a casos conocidos que presenten similitud, estableciendo semejanzas, diferencias y predicciones. Al utilizar analogías como recurso educativo se debería plantear al alumno una situación nueva en el marco de un núcleo problemático e incentivarlo a recurrir a conocimientos previos para su indagación. La creación de modelos recurriendo al campo lógico-matemático también constituye un recurso analógico de mucha utilidad para la comprensión de conceptos complejos, facilitando la interdisciplinariedad y la transdisciplinariedad. Cuanto mayor sea la conciencia de estar recurriendo a analogías, tanto por parte del docente como de los estudiantes, mayor será el valor epistémico que se les otorgue en la construcción del conocimiento aprendido. Este tipo de enseñanza enriquece la creatividad -como capacidad de descubrir y de resolver problemas-, facilita la comprensión -como transferencia de lo teórico a situaciones nuevas-, mejora la retención de lo aprendido y su reutilización futura, y favorece la autonomía del alumno. Se postula que recurrir explícitamente a las analogías como recurso para la enseñanza de las ciencias elevará la calidad educativa y formará profesionales con mayores competencias para la resolución de problemas y la generación de conocimientos. En este trabajo se analizaron casos de enseñanza de la Paleontología en interacción con otras disciplinas.

*Proyecto financiado por PI-UNRN-40-A-620.

\section{QUISTES DE DINOFLAGELADOS DEL MIEMBRO AGUA DE LA MULA (FORMACIÓN AGRIO), CRETÁCICO INFERIOR DE CUENCA NEUQUINA, ARGENTINA}

\author{
M.A. Paolillo ${ }^{1}$, M.V. Guler ${ }^{1}$, M. Lescano ${ }^{2}$, A. Concheyro ${ }^{2}$ y M.B. Aguirre-Urreta ${ }^{2}$
}

${ }^{1}$ Instituto Geológico del Sur (INGEOSUR), Consejo Nacional de Investigaciones Científicas y Tecnológicas (CONICET)-Universidad Nacional del Sur. San Juan 670, B8000ICN Bahía Blanca, Buenos Aires, Argentina. meli_bal88@hotmail.com; vguler@criba.edu.ar

IInstituto de Estudios Andinos 'Don Pablo Groeber' (IDEAN), Consejo Nacional de Investigaciones Científicas y Tecnológicas (CONICET)-Facultad de Ciencias Exactas y Naturales, Universidad de Buenos Aires. Intendente Güiraldes 2160, Pabellón II, Ciudad Universitaria, C1428EGA Ciudad Autónoma de Buenos Aires, Argentina.lescano@gl.fcen.uba.ar; andrea@gl.fcen.uba.ar; aguirre@gl.fcen.uba.ar

En el presente trabajo se aborda el estudio bioestratigráfico de los quistes de dinoflagelados de pared orgánica (dinoquistes) del Miembro Agua de la Mula (Hauteriviano superior) de la Formación Agrio en la localidad de Mina San Eduardo. Dicha sección se sitúa en la parte central de la Cuenca Neuquina, en el norte de la provincia de Neuquén. Se reconocieron un total de 58 taxones de dinoquistes, todos representantes del Orden Gonyaulacales, pertenecientes en su mayoría a la Familia Gonyaulacaceae con algunos géneros propios de las Familias Aeroligeraceae y Ceratiaceae. Las asociaciones se componen mayoritariamente de taxones cosmopolitas de extensos rangos estratigráficos tales como: Circulodinium distinctum, Oligosphaeridum complex, Kiokansium unituberculatum, Dingodinium cerviculum y Florentinia mantelli entre otros. Se identificaron además tres especies bioestratigráficamente significativas para el Cretácico Inferior, tales como Bourkidinium granulatum, B. elegans, Callaiosphaeridium asymmetricum y Cymososphaeridium validum. Los bioeventos de últimas ocurrencias de las cuatro especies se registraron en muestras consecutivas en la parte superior de la Biozona de amonites Paraspiticeras groeberi del Hauteriviano tardío. La comparación con los registros mediterráneos de B. granulatum, B. elegans y C. validum indican un alto grado de isocronía con los datos obtenidos para estas especies en la cuenca. Por otra parte, se espera aún confirmar el registro exacto de última aparición de C. asymmetricum para la Cuenca Neuquina en futuros estudios en diferentes secciones. En general, las asociaciones de dinoquistes así como las especies diagnósticas seleccionadas en el miembro Agua de la Mula exhiben clara afinidad con aquellas de la región mediterránea. 


\title{
CYCLOCARDIA CONRAD, ONE OF THE MOST DIVERSE GENERA OF CARDITIDS (BIVALVIA: CARDITIDAE): A MONOPHYLETIC GROUP OR A WASTEBASKET TAXON?
}

\author{
D.E. PÉREZ $Z^{1}$ \\ 'Museo Argentino de Ciencias Naturales "Bernardino Rivadavia" (MACN)-Consejo Nacional de Investigaciones Científicas y Tecnológicas \\ (CONICET). Av. Ángel Gallardo 470, C1405DJR Ciudad Autónoma de Buenos Aires, Argentina. trophon@gmail.com
}

More than a hundred species were assigned to the genus Cyclocardia in the bibliography, including living and fossil taxa covering a long timelapse (Eocene-Recent) and wide geographical range (South and North America, East Russia, Antarctica, and Europe). Previous phylogenetic and systematic analyses suggested the non-monophyly of this taxon. In this work, phylogenetic and morphospace analyses of several species of Cyclocardia and other related taxa are performed using morphological shell-characters, and including fossil and living species (94 taxa and 147 characters). In all the searches (using equal and implied weighting) Cyclocardia results a polyphyletic group. A monophyletic Cyclocardia is composed only by C. borealis (type species of Cyclocardia), C. novangliae and C. compressa (all living Atlantic species). The previously proposed genera Ainicardita, Lunulicardita and Crassicardia are all monophyletic groups in most of the searches (with Ainicardita as part of the planicostate lineage). European species of (yclocardia fall in the genus Scalaricardita. The proposed group "awamoensis" for New Zealand and South American fossil Cyclocardia is paraphyletic in most of the searches. A clade composed by C. hamiltonensis, C. yakatagensis, and C. barbarensis (all taxa from the North Pacific) is obtained in all the searches. When a monophyletic Cyclocardia is constrained the resulting topology comprises 49 more steps in equal weighted searches. Morphospace analysis shows a Cyclocardia group with a wide occupation of the morphospace, at least as large as the remaining carditids, but restricted to a defined portion of this. Therefore, "Cyclocardia" group is a morpho-logical grade rather than a monophyletic clade.

\section{MORPHOLOGICAL DISPARITY THROUGH TIME OF CALLIOTROPIS(VETIGASTROPODA: EUCYCLIDAE)}

\author{
D.E. PÉreZ ${ }^{1}$, S.M. FerRari ${ }^{2}$ y M.D. EZCURRA ${ }^{1}$
}

${ }^{1}$ Museo Argentino de Ciencias Naturales "Bernardino Rivadavia" (MACN)-Consejo Nacional de Investigaciones Científicas y Tecnológicas
(CONICET). Av. Ángel Gallardo 470, C1405DJR Ciudad Autónoma de Buenos Aires, Argentina. trophon@gmail.com; martindezcurra@yahoo.com.ar
2Instituto Patagónico de Geología y Paleontología (IPGP), Centro Nacional Patagónico (CENPAT)-Consejo Nacional de Investigaciones Cientí-
ficas y Tecnológicas (CONICET). Boulevard Brown 2915, U9120ACD Puerto Madryn, Chubut, Argentina. mferrari@cenpat-conicet.gob.ar Calliotropis Seguenza is a genus of marine gastropods ranging from the Triassic to Recent, in which modern forms are strikingly similar to Mesozoic species. Previous authors suggest a distinction between living and fossil representatives, with possible paleoecological differences (shallow/deep waters, respectively). A recent quantitative phylogenetic analysis of Calliotropis (36 terminals and 85 shell-characters) allows performing a morphological disparity analysis of the group based on discrete characters. A distance matrix was calculated from the phylogenetic matrix using maximum observable rescaled distances and the weighted mean pairwise dissimilarity was used as the pre-ordination disparity metric. We analyzed morphological changes through the entire biochron of the clade using three character-sets: the first using all characters, the second using only characters with paleoecological signal, and the third using characters excluded from the second approach. Three clusters are clearly separated in the morphospace using all the characters, representing Triassic, JurassicPaleogene and Neogene-Recent species, respectively. Disparity curves through time show non-significant differences across time-bins but morphospace occupation shows significant shifts from the Triassic to Jurassic and from the Paleogene to Neogene. The Paleogene group has a significantly larger morphospace than Neogene species. The approaches using only characters with paleoecological signal or without them found results very similar to those using all characters. From these results, we can preliminary interpret that the morphological differences observed within Calliotropis along the geological record may not be driven by paleoecological constraints, but by intrinsic phenotypic evolution of the group through time. 


\title{
LOS ROEDORES CAVIIDAE DE LAS HUAYQUERÍAS DEL ESTE (MIOCENO TARDÍO-PLIOCENO?) DE LA PROVINCIA DE MENDOZA (ARGENTINA)
}

\author{
M.E. Pérez ${ }^{1}$, M.C. Madozzo-Jaen ${ }^{1}$, A. Forasiepl ${ }^{2}$, F. Prevosti ${ }^{3}$ y A. Garrido ${ }^{4}$

\begin{abstract}
1'Museo Paleontológico Egidio Feruglio. Avenida Fontana 140, U9100GYO Trelew, Chubut, Argentina.mperez@mef.org.ar;
\end{abstract} \\ cmadozzojaen@mef.org.ar \\ ${ }^{2}$ Instituto Argentino de Nivología, Glaciología y Ciencias Ambientales (IANIGLA), Centro Científico Tecnológico Mendoza-Consejo Nacional de \\ Investigaciones Científicas y Tecnológicas (CONICET). Avenida Ruiz Leal s/n, 5500 Mendoza, Mendoza, Argentina. borhyaena@hotmail.com \\ ${ }^{3}$ Centro Regional de Investigaciones Científicas y Transferencia Tecnológica de La Rioja (CRILAR)-Universidad Nacional de La Rioja-Servicio \\ Geológico Minero Argentino (SEGEMAR)-Universidad Nacional de Catamarca-Consejo Nacional de Investigaciones Científicas y Tecnológicas \\ (CONICET). Entre Ríos y Mendoza s/n, 5301 Anillaco, La Rioja, Argentina. protocyon@hotmail.com \\ "Museo Provincial de Ciencias Naturales "Prof. Dr. Juan A. Olsacher"-Dirección Provincial de Minería. Etcheluz y Ejercito Argentino, 8340 \\ Zapala, Neuquén, Argentina. albertocarlosgarrido@gmail.com
}

En las Huayquerías de Mendoza, las Formaciones Huayquerías y Tunuyán conforman una sucesión de edad Mioceno Tardío (U/Pb $5.84 \pm 0.41 \mathrm{Ma}$ )-Plioceno?, alcanzando una potencia (expuesta) de unos $290 \mathrm{~m}$. Estas unidades se presentan en concordancia, evidenciándose en la base de la Formación Tunuyán un rejuvenecimiento del área de aporte materializado por la irrupción de camadas conglomerádicas fluviales. Hasta el momento, los cávidos conocidos para esta localidad estaban representados por Cardiomys ameghinorum Rovereto y Prodolichotis mendocina Rovereto recuperados de la Formación Tunuyán. El objetivo de esta comunicación es dar a conocer nuevos restos de cávidos hallados en recientes campañas en las denominadas Huayquerías del Este. Los especímenes pertenecen a la colección del IANIGLA. El análisis taxonómico de dichos ejemplares permitió reconocer para la Formación Huayquerías y base de la Formación Tunuyán los siguientes taxa: Neocavia lozanoi, Palaeocavia sp. nov., Dolichotinae sp., Cardiomys? sp. Por otro lado, Prodolichotis mendocina y Orthomyctera andina Rovereto se registran además en la Formación Tunuyán. Un morfotipo diferente de Palaeocavia se registra solo en la Formación Tunuyán. Como resultado inmediato, se registra una mayor diversidad de caviomorfos conocidos para las Huayquerías de Mendoza. A nivel evolutivo se corrobora, a través de los nuevos taxones, el pulso de diversificación inferido en trabajos previos para Caviidae durante el Mioceno Tardío. Como consecuencia biogeográfica, se observa una mayor similitud especifica entre las Huayquerías de Mendoza y el noroeste argentino (NOA), mientras que a nivel genérico también comparte taxones con la Formación Cerro Azul (La Pampa) y el noreste argentino (NEA).

\section{NUEVOS HALLAZGOS PALINOLÓGICOS EN LA FORMACIÓN RÍO MAYER (CRETÁCICO INFERIOR) EN LA ZONA DE RÍO LISTA, PROVINCIA DE SANTA CRUZ, ARGENTINA}

\author{
V.S. Perez Loinaze ${ }^{1}$, M. Llorens'², J.C. Villan³ Y J.I. CUitiño ${ }^{4 *}$ \\ ${ }^{1}$ Museo Argentino de Ciencias Naturales "Bernardino Rivadavia" (MACN)-Consejo Nacional de Investigaciones Científicas y Tecnológicas \\ (CONICET)". Av. Ángel Gallardo 470, C1405DJR Ciudad Autónoma de Buenos Aires, Argentina. loinazev@gmail.com \\ ${ }^{2}$ Facultad de Ciencias Naturales, Universidad Nacional de la Patagonia San Juan Bosco. 9 de Julio 25, U9100CKN Trelew, Chubut, Argentina. \\ mllorens@conicet.gov.ar \\ ${ }^{3}$ Departamento de Ciencias Geológicas, Facultad de Ciencias Exactas y Naturales, UBA. Intendente Güiraldes 2160, Ciudad Universitaria, \\ Pabellón II, C1428EGA Ciudad Autónoma de Buenos Aires, Argentina. juancruzvillan11@gmail.com \\ ${ }^{4}$ Instituto Patagónico de Geología y Paleontología, Centro Nacional Patagónico (CENPAT)-Consejo Nacional de Investigaciones Científicas \\ y Tecnológicas (CONICET). Boulevard Brown 2915, U9120ACD Puerto Madryn, Chubut, Argentina. jcuitino@cenpat-conicet.gob.ar
}

Se analiza el contenido palinológico de cinco niveles fértiles (BA Pal 6552/6556) pertenecientes a la Formación Río Mayer en la zona del Río Lista, provincia de Santa Cruz, Argentina. Las palinofloras recuperadas fueron obtenidas de potentes bancos pelíticos depositados en un ambiente marino de plataforma externa de baja energía a costero. Se identificaron 23 especies de esporas y 17 de granos de polen de gimnospermas, los granos de polen de angiospermas están escasamente representados y son referidos a Clavatipollenites sp. También se observa la presencia de abundantes elementos marinos, representados por los géneros Pterospermella, Michrystridium y Veryhachium, abundantes quistes de dinoflagelados y microforaminíferos. Aproximadamente el 50\% de las especies reconocidas no habían sido previamente identificadas para la unidad. En todos los niveles analizados los elementos más abundantes son los granos de Classopollis spp. relacionados con cheirolepidiáceas y los diversos elementos marinos. Además, el análisis cuantitativo de las asociaciones palinológicas recuperadas permite realizar inferencias paleoambientales. Los elementos marinos alcanzan $52 \%$ de la microflora en los niveles basales y Classopollis spp. alcanza 43,2\%. En los niveles superiores esta relación se invierte, disminuyendo la participación de los elementos marinos en la asociación al 17\%, mientras que Classopollis spp. alcanzan el 70\%. En base a estos valores es posible inferir una progresiva somerización del ambiente de sedimentación.

*Proyecto financiado por PIP 286. 


\title{
REDESCUBRIENDO LA COLECCIÓN PALEONTOLOGICA DEL MUSEO INCA HUASI (LA RIOJA- ARGENTINA) MEDIANTE PROYECTOS DE EXTENSIOON UNIVERSITARIA
}

\author{
S. Pérez Parry ${ }^{1}$ y M.D.L.A. MezA ${ }^{1}$ \\ 1Parque Geológico Sanagasta. Ruta 75, km 34, 5301 Villa Sanagasta, La Rioja, Argentina. sebastianperezparry@gmail.com; \\ mezamariadelosangeles@gmail.com
}

Las prácticas de estudio dentro de la cátedra Museografía 1, de la Tecnicatura Universitaria en Museología de la Universidad Nacional de La Rioja, permiten a los alumnos realizar investigaciones en las colecciones de los museos de La Rioja. En el año 2014, en una de estas visitas, los autores encontraron restos fósiles en la colección del Museo Arqueológico Inca Huasi. Los mismos pertenecerían a un Megatherium, una especie nunca descripta en la provincia de La Rioja, por lo tanto, desconocida en el ámbito científico. Durante tres años se realizaron tareas de búsqueda y recolección de metadatos capaces de asociar estos restos fósiles al contexto de su descubrimiento. Mediante fotografías, recortes de diarios, entrevistas e inventarios obtenidos en diversos ámbitos, se pudo reconstruir no solo el contexto del hallazgo, que databa de 1958, sino también la ubicación exacta donde se hallaron los restos. Con esta información, los autores se contactaron con el paleontólogo del Centro Regional de Investigaciones Científicas y Transferencia Tecnológica de La Rioja, Francisco Prevosti para concretar una visita al yacimiento ubicado en Olta, La Rioja, y para realizar la puesta en valor del material redescubierto en el ámbito científico. Actualmente las tareas de acondicionamiento de la colección paleontológica continúan mediante proyectos multidisciplinares de extensión universitaria, desarrollando divulgación hacia la comunidad de La Rioja, involucrando museólogos, paleontólogos, técnicos, estudiantes y graduados.

\section{NUEVA INFORMACIÓN PALINOLÓGICA PARA LA FORMACIÓN ALLEN (CRETÁCICO SUPERIOR) EN LA LOCALIDAD LAGO PELLEGRINI, PROVINCIA DE RÍO NEGRO, ARGENTINA}

\author{
E.V. Pérez Pincheira ${ }^{1}$ y M. Di Pasquo ${ }^{1}$
}

${ }^{1}$ Laboratorio de Palinoestratigrafía y Paleobotánica, Centro de Investigaciones Científicas y Transferencia de Tecnología a la Producción (CICYTTP), Consejo Nacional de Investigaciones Científicas y Tecnológicas (CONICET)-Entre Ríos, Universidad Autónoma de Entre Ríos (UADER). Materi y España, E3105BWA Diamante, Entre Ríos, Argentina. eglysauria@hotmail.com; medipa@cicyttp.org.ar

Se investiga el contenido palinológico de la Formación Allen en el sector conocido localmente como Tierras Blancas (38 $47^{\prime}$ 25.4" S; 68 00' 43.8" O), donde se muestreó una sección heterolítica de ca. 10 m, la cual se inicia sobre base cubierta con un banco de pelita (bentonita) verdosa y continúa con bancos de areniscas finas claras y pelitas macizas y laminadas grises y verdosas. En su parte inferior son frecuentes finas intercalaciones de yeso y a 6 m de la base se identificó un nivel con briznas y restos vegetales no identificados. Se tomaron ocho muestras de niveles pelíticos, de los cuales cuatro resultaron fértiles en el tramo inferior presentando palinomorfos en su mayoría enteros, con buen estado de preservación y con baja alteración térmica. Se registra un predominio de especies continentales tales como esporas de Bryophyta, Lycophyta y Pteridophyta (Ruffordiaspora australiensis, Cyathidites minor, Clavifera triplex), granos de polen de gimnospermas (Callialasporites dampieri, C. trilobatus, Classopollis classoides, Microcachryidites antarcticus, Podocarpidites marwickil) y angiospermas (Liliacidites, Peninsulapollis gilli), algas verdes (Botryococcus, Pediastrum boryanum), formas esferoidales atribuibles a zygosporas y quistes de dinoflagelados peridinioideos pequeños, translúcidos, ¿dulceacuícola?. Por la presencia de $C$. dampieri, $P$. gilliy R. australiensis la asociación en la Formación Allen es atribuida al Campaniano-Maastrichtiano. Esta asociación es correlacionada con la palinoasociación 1 asignada al Miembro Medio de la Formación Allen en el Cerro Gutiérrez (6 km hacia el Oeste), pues comparten más del 70\% de los taxones, entre ellos R. australiensis, C. triplex, C. trilobatus, C. dampieri, C. classoides, P. gilli, formas algales y dinoflagelados peridinoideos. 


\title{
HISTORIA DE LA TIERRA: LÍNEA DE TIEMPO
}

\author{
J. PICKLING ${ }^{1}$ Y A. VILLASECA ${ }^{1}$
}

1Museo de Historia Natural Vera Alleman, Universidad Ricardo Palma. Avenida Benavides 5440, Lima, Santiago de Surco, Perú.mhn@urp.edu.pe; a.villaseca.r@outlook.com

El principal objetivo de este trabajo fue presentar de manera didáctica la historia de la vida en la Tierra en $46 \mathrm{~m}$ donde $1 \mathrm{~cm}$ representa un millón de años. La metodología se basó en stands de madera de $20 \mathrm{~cm}$ ancho x $230 \mathrm{~cm}$ de largo y $120 \mathrm{~cm}$ de alto; acompañados de imágenes e información explicativa; los eones se marcaron en el suelo con carteles color amarrillo; las maquetas que se expusieron fueron diseñadas por el paleoartista Jose Pickling; entre las cuales tenemos fauna local peruana como el pingüino Icadyptes salasi y mundial representada por pterosaurio Tapejara imperator y el plesiosaurio Liopleurodon ferox, entre otros. Como resultado, la dinámica se presentó en diferentes lugares de Lima y fue visita por público de todas las edades. Se rescató la opinión de los asistentes por consulta personal verbal de lo cual se destaca el asombro por el poco conocimiento del tiempo geológico, momento de aparición de la vida en la Tierra, desconocimiento de mega continentes anteriores a Pangea y más de una extinción masiva a lo largo de todos los tiempos geológicos. La presente dinámica puede ser montada en diferentes instituciones: colegios, institutos, universidades y tiene como función primordial divulgar la historia de la Tierra.

\section{LINKING PRIMARY PRODUCTIVITY AND BENTHIC COMMUNITY SHIFTS IN SALTO GRANDE RESERVOIR}

\author{
M.S. Plastani ${ }^{1}$, C. Laprida ${ }^{1}$, D. Gangl ${ }^{2}$, A. Lami ${ }^{3}$, and P. De Tezanos Pintos ${ }^{4}$ \\ ${ }^{1}$ Laboratorio de Sondeos de Ambientes Continentales y Marinos (SACMa) - Instituto de Estudios Andinos "Don Pablo Groeber" (IDEAN), Facultad \\ de Ciencias Exactas y Naturales, Universidad de Buenos Aires-Consejo Nacional de Investigaciones Científicas y Tecnológicas (CONICET). Intendente \\ Güiraldes 2160, C1428EGA Ciudad Autónoma de Buenos Aires, Argentina.splastani@gl.fcen.uba.ar; chechulaprida@gmail.com \\ 2Laboratorio de Limnología, Instituto de Ecología, Genética y Evolución de Buenos Aires. Universidad de Buenos Aires-Consejo Nacional de In- \\ vestigaciones Científicas y Técnicas. Intendente Güiraldes 2160, C1428EGA Ciudad Autónoma de Buenos Aires, Argentina.gangi@agro.uba.ar \\ ${ }^{3}$ Instituto per lo Studio degli Ecosistemi, CNR. Largo Tonolli 50, 28922 Verbania, Pallanza, Italia. a.lami@ise.cnr.it \\ 4Instituto de Botánica Darwinion (IBODA), Academia Nacional de Ciencias Exactas, Físicas y Naturales (ANCEFN). Labardén 200, B1642HYD \\ San Isidro, Buenos Aires, Argentina.paulatezanos@yahoo.com
}

The Salto Grande Hydroelectric Complex was built in 1979 in the course of the Uruguay river, upriver from the cities of Concordia (Argentina) and Salto Grande (Uruguay). Since year 2000, the Reservoir has been suffering recurrent blooms of cyanobacteria. In this study we analyze for the first time sedimentary pigments from the sediments of its reservoir in order to reconstruct phytoplankton communities prior to regular monitoring efforts initiated in year 2000. Based on this paleolimnological archive and a well-established 210Pb chronology, we aimed to analyze if the phytoplankton composition has changed significantly during the last decades, and which could have been the main environmental drivers that lead cyanobacteria blooms. During the first 20 years after inundation, diatoms, cryptophytes, chlorophytes and cyanobacterias dominate the phytoplankton communities. An abrupt change in productivity occurred after ca. 2003, but with no major shift in phytoplankton composition. This change is reflected in benthic communities: the increase in pigments belonging to sulphurphotosynthetic anoxygenic bacteria (i.e., green sulphur and purple sulfur bacteria) suggest a marked lowering in the oxygen concentrations. The causes of dysoxia are still elusive, but the interplay between climatic tendencies promoting water stratification especially during summers, and enhanced carbon flux related to higher phytoplankton productivity, could have increased the oxygen demand: the utilization of metabolizable organic matter could have exceeded the rate of oxygen supply due to stratification, favoring dysoxia. This work highlights the potential of the paleolimnological approach for disentangling modern ecological trajectories in absence of monitoring programs. 


\title{
TWO NEW CAECULID MITES FROM THE CRETACEOUS AMBER OF MYANMAR, WITH NOTES ON THE PHYLOGENY OF THE FAMILY CAECULIDAE (ACARI: PROSTIGMATA)
}

\author{
A.O. Porta ${ }^{1}$, D.N. Proud², E.H. Franchi ${ }^{3}$, M.B. Epele ${ }^{4}$, P. Michalik ${ }^{5}$, M.A. Morvidone ${ }^{6}$, and W. Porto ${ }^{1}$ \\ 1Divisón Aracnología, Museo Argentino de Ciencias Naturales. Av. Ángel Gallardo 470, C1405DJR Ciudad Autónoma de Buenos Aires, Argentina. \\ hugporta@yahoo.com.ar; w.insecta@gmail.com \\ 2Department of Biological Sciences,Moravian College. 1200 Main Street, PA 18018, Bethlehem, Pennsylvania, United States of America. \\ dnp0646@gmail.com \\ ${ }^{3}$ División Icnología, Museo Argentino de Ciencias Naturales. Av. Ángel Gallardo 470, C1405DJR Ciudad Autónoma de Buenos Aires, Argentina. \\ ezequielfranchi@hotmail.com \\ ${ }^{4}$ Laboratorio de Métodos Analíticos, YPF Tecnología (Y-TEC). Avenida del Petróleo s/n, 1923 Berisso, Buenos Aires, Argentina. \\ maria.epele@ypftecnologia.com \\ ${ }^{5}$ Zoological Museum, University of Greifswald. Loitzer Str. 26, 17489, Greifswald, Mecklenburg-Vorpommern, Germany. \\ michalik@uni-greifswald.de \\ שUniversidad de San Martín. 25 de Mayo y Francia, 1650 San Martín, Buenos Aires, Argentina. morvidone@gmail.com
}

At present only three species of caeculid mites, all belonging to the genus Procaeculus Jacot, have been reported from amber: P. eridasonae Coineau and Magowski, from Baltic amber, P. dominicensis Coineau and Poinar, from Dominican amber (Upper Eocene) and Procaeculus sp. Rivas, Serrano-Sánchez and Vega, from Chiapas amber (Early Miocene). In this work we describe two new species of caeculid mites, including the first from Burmese amber (Upper Cretaceous). Based on a phylogenetic analysis of the family, both species are included in the genus Procaeculus, which is redefined herein. Descriptions are accompanied by illustrations, photographs and 3D reconstructions.

\section{BONE MICROSTRUCTURE IN PROTEROCHAMPSIA FROM THE CHAÑARES FORMATION}

\author{
E. Previtera ${ }^{1}$, A.C. Mancuso ${ }^{1}$ y A.B. Arcuccl ${ }^{2 *}$
}

${ }^{1}$ Instituto Argentino de Nivología, Glaciología y Ciencias Ambientales (IANIGLA), Centro Científico Tecnológico Mendoza-Consejo Nacional de Investigaciones Científicas y Tecnológicas (CONICET). Avenida Ruiz Leal s/n, 5500 Mendoza, Mendoza, Argentina.

eprevitera@mendoza-conicet.gov.ar; amancu@mendozaconicet.gov.ar

2Área de Zoología, Instituto Multidisciplinario de Investigaciones Biológicas, Universidad Nacional de San Luis. Chacabuco 917, D5700HHW San Luis, San Luis, Argentina. andrea.arcucci@gmail.com

Proterochampsids are South American endemic non-archosaurian archosauriforms have a restricted temporal distribution, and which origin, diversification and disappearance happened in a very short period of time in the Late Triassic. Some contributions about the anatomical and phylogenetic knowledge of proterochampsids have been published, but paleobiological hypothesis about the group are still scarse. We developed detailed histological descriptions and evaluated paleobiological hypotheses about the Chañares proterochampsids. For this purpose, seven thin sections of long bones belonging to six proterochampsian specimens were analyzed. These show proterochampsian diagnostic characters, but they did not display autopomorphies that allow us different among the Chañares taxa. The histological examination revealed a predominance of fibrolamellar tissue suggesting a rapid periosteal osteogenesis, and therefore an overall fast bone growth. The absence of large amounts of mature secondary osteons is comparable with that recently observed in aquatic non-phylogenetically related placodonts. The existence of discontinuities (LAGs) expressed the capability to rapid response to environmental changes. The proterochampsid samples display higher growth rates than those reported in pseudosuchians and other basal archosauromorphs, resembling some dinosauromorphs, as well as some basal dinosaurs.

*Financial support by PICT 2013-0805 to Adriana C. Mancuso. 


\title{
FIRST RECORD OF SEXUAL DIMORPHISM IN THE GENUS ANAGAUDRYCERAS (AMMONOIDEA, LYTOCERATOIDEA) FROM ANTARCTICA
}

\author{
M.E. RAFFI ${ }^{1}$, AND E.B. Olivero ${ }^{1 *}$
}

${ }^{1}$ Laboratorio de Geología Andina, Centro Austral de Investigaciones Científicas (CADIC)-Consejo Nacional de Investigaciones Científicas y Tecnológicas (CONICET); Instituto de Ciencias Polares, Ambiente y Recursos Naturales (ICPA), Universidad Nacional de Tierra del Fuego, Antártida e islas del Atlántico Sur. B. Houssay 200, V9410CAB Ushuaia, Tierra del Fuego, Argentina. eugeniaraffi@gmail.com; emolivero@gmail.com

As a result of ongoing systematic studies in the late-early to mid Campanian Rabot Formation, two adult morphotypes of the genus Anagaudryceras have been recorded. They coexist in the last $50 \mathrm{~m}$ of the Member II, Ammonite Assemblage 6 , Natalites spp. Group 2 and the beginning of the Member III, Ammonite Assemblage 7 Neokosmaticeras redondensis. These morphs are associated with Baculites subanceps Haughton, Metaplacenticeras subtilistriatum Jimbo and Hoplitoplacenticeras sp., which indicate elsewhere the base of the mid Campanian. The adult body chamber of the bigger and compressed morphotype $A(\mathrm{~Wb} / \mathrm{Wh} \sim 0.80$ ) has band-like ribs preceded by a marked constriction that runs parallel to the rib; the adult body chamber of morphotype $B$ is depressed (Wb/Wh 1.18-1.03) and has two types of ornamentation; strong asymmetrical, slightly flexuous ribs, preceded by constrictions and almost imperceptible spiral striations. These adult morphotypes develop from morphologically similar depressed juvenile shells. The neanoconch is ornamented by five or less ribs accompanied by fine lirae. The rest of the phragmocone has fine, flexuous, prorsiradiate lirae and up to five collars per whorl. As these two morphotypes are characterized by a) differences of ornamentation only in the adult body chamber, b) similar abundance of specimens and c) same stratigraphic range, we conclude that they represent an Anagaudryceras antidimorphic pair. This is the first evidence of sexual dimorphism in Anaguadryceras; even though no peristome or lappets are preserved, we tentatively differentiated the morphotype $A$ as the macroconch [ㅇ] and the morphotype B as the microconch [ $\hat{O}$ ].

*Financial support by PIDUNT dF-A1.

\section{"PROLAGOSTOMUS AMPLUS" AMEGHINO ES UN SINÓNIMO JUNIOR DE PLIOLAGOSTOMUS NOTATUS AMEGHINO (RODENTIA, CHINCHILLIDAE; MIOCENO TEMPRANO DE SANTA CRUZ, ARGENTINA)}

\section{L.L. RASIA ${ }^{1}$ Y A.M. CANDELA ${ }^{1}$}

1División Paleontología Vertebrados, Museo de La Plata, Facultad de Ciencias Naturales y Museo, Universidad Nacional de La Plata. Paseo del Bosque s/n, B1900FWA La Plata, Buenos Aires, Argentina. lucianorasia@fcnym.unlp.edu.ar; acandela@fcnym.unlp.edu.ar

Chinchillidae es un grupo de roedores caviomorfos que incluye a los géneros vivientes Chinchilla, Lagidium y Lagostomus. Este último, cuya única especie viviente es Lagostomus maximus, se agrupa en la subfamilia Lagostominae junto con los géneros extintos Eoviscaccia (Oligoceno Temprano-Mioceno Temprano), Prolagostomus y Pliolagostomus (Mioceno Temprano-Medio). Los lagostominos están representados en la Formación Santa Cruz (Mioceno Temprano de Santa Cruz, Argentina) por Pliolagostomus notatus y ocho especies de Prolagotomus. La especie "Prolagostomus amplus" fue originalmente caracterizada por poseer todos los molariformes inferiores del mismo tamaño. El análisis detallado del holotipo y material referido indica que "Prolagostomus amplus" carece de varios de los rasgos comúnmente utilizados para diferenciar Prolagostomus del resto de los lagostominos (e.g., molares con hipoflexo/hipofléxido sinuoso, caras anterior y lingual de los molares inferiores no forma un ángulo marcado, tercer lóbulo del M3 de forma laminar y posterior o posterolingualmente orientado). A su vez, el material comparte con Pliolagostomus notatus la presencia de hipoflexos/hipofléxidos rectos, caras anterior y lingual de los molares inferiores formando un ángulo marcado, tercer lóbulo del M3 de forma subtriangular y lingualmente orientado, foramen mentoniano ubicado dorsolateralmente en la mandíbula. Por lo tanto consideramos a "Prolagostomus amplus" un sinónimo junior de Pliolagostomus notatus. Un análisis filogenético indicó que Pliolagostomus, Prolagostomus y Lagostomus se agrupan en el clado Lagostominae, que está más cercanamente relacionado con los Chinchillinae vivientes Chinchilla y Lagidium que con el género extinto Eoviscaccia. 


\title{
VISIBILIZACIÓN DE LA RECONSTRUCCIÓN COMO RECURSO PEDAGÓGICO
}

\author{
S. REUIL ${ }^{1}$ \\ ${ }^{1}$ santiagoreuil@gmail.com
}

La reconstrucción de un espécimen fósil fragmentario no solo es una forma de presentar un hallazgo, sino también una interpretación del ejemplar. Muchos fósiles, por informativos que sean, pueden ser muy difíciles de entender para el ojo no entrenado. Mediante el estudio y la comparación llevada a cabo por especialistas, se puede hacer una especulación fundamentada sobre las partes faltantes y hacer una reconstrucción que sea más accesible al público general. Es frecuente en las reconstrucciones de dinosaurios que a las áreas reconstruidas se les de una textura y color que imitan las del fósil. De este modo la reconstrucción simula ser una pieza "completa" dando a entender que todo lo que vemos es "evidencia" y no interpretación. Otra variante, frecuente en las reconstrucciones paleoantropológicas, es reconstruir las partes faltantes con una terminación y colores que se diferencian claramente de las porciones preservadas. Este trabajo recupera esta última propuesta considerándola de mayor valor didáctico por brindar más niveles de lectura para el ojo interesado. Hace explícito que hay partes que son evidencia y partes que son interpretación; que la evidencia es fragmentaria. Muestra que hay un trabajo y un estudio detrás de la pieza invitando a preguntarse por el proceso: alguien lo hizo así, ¿Por qué? ¿En qué se basó? ¿Podría ser diferente? Es una pieza que invita a ser cuestionada y no tomada como definitiva. Es una forma de mostrar el conocimiento como una estructura abierta, no cerrada. Una manera de mostrar el proceso y no solo el resultado.

\section{PLIOCENE-HOLOCENE CALCAREOUS NANNOFOSSILS FROM THE MALVINAS TROUGH, SOUTHWESTERN ATLANTIC OCEAN}

\author{
L. RIVAS ${ }^{1}$, J.P. PÉREZ PANERA ${ }^{1}$, AND G. CUSMINSKY ${ }^{2 *}$
}

1YPF Teconología. Av del Petróleo s/n, 1923 Berisso, Buenos Aires, Argentina. lucia.rivas@ypftecnologia.com; juan.p.panera@ypftecnologia.com ${ }^{2}$ Instituto de Investigaciones en Biodiversidad y Medioambiente (INIBIOMA), Consejo Nacional de Investigaciones Científicas y TecnológicasUniversidad Nacional del Comahue (CONICET-UNCO). Quintral 1250, R8400FRF San Carlos de Bariloche, Río Negro, Argentina.

gcusminsky@gmail.com

Preliminary results on calcareous nannofossils from the 13-14/2 piston core in the Malvinas Trough, Southwestern Atlantic Ocean (SAO), are presented. Previous works in SAO deal with radiolarians, planktic and benthic foraminifera. Only few studies were focused on the Pliocene-Holocene nannofossil record, and they are restricted to the Ewing Bank and Malvinas Plateau. Sediment of the 19 samples studied are calcareous silty clays (lower and middle part), and sandy-silts (upper part). Along the entire core, we recorded high abundance of radiolarians and diatoms. Calcareous nannofossil assemblages are characteristic of cold surface waters. The lower section yields a moderately diverse assemblage dominated by Reticulofenestra pseudoumbilicus and R. minuta, R. minutula, R. haqii, Calcidiscus macintyrei, Coccolithus pelagicus, Pseudoemiliania lacunosa, P. ovata and Sphenolithus moriformis as accompanying taxa. The coexistence of R. pseudoumbilicus, R. haqii, P. ovata and $P$. lacunosa indicates a Pliocene age (NN15 biozone) for this assemblage. Overlaying this level, the sedimentary deposit of the middle part is barren of calcareous microfossils and is coincident with a marked change in the lithology and sedimentary structures and color. These levels are interpreted as the record of a major paleoclimatic glacial event that raised the CCD due to a northern migration of the Polar Front. The upper part of the core shows an assemblage characterized by Emiliania huxleyi and Gephyrocapsa spp. Based on the first occurrence of E. huxleyi, this upper part corresponds to the NN21 biozone, latest Pleistocene to Holocene. 


\title{
THE RECORD OF OSTRACOD GENUS HARPABOLLIA SCHALLREUTER IN UPPER ORDOVICIAN LEVELS OF THE PARANÁ BASIN, BRAZIL: ITS PALEOBIOGEOGRAPHIC IMPLICATIONS
}

\author{
M.J. SALAS'1 L. REILY², R.R. AdôRnO², AND D.A. Do CARMO²
}

${ }^{1}$ Centro de Investigaciones en Ciencias de la Tierra, (CICTERRA). Av. Vélez Sársfield 1611, 5000 Córdoba, Córdoba, Argentina.mjsalas@unc.edu.ar 2Laboratory of Micropaleontology, University of Brasília. Campus Darcy Ribeiro, 70910-900 Brasilia, DF, Brasil. livioeueu@gmail.com; rodrigo-afonso@cprm.gov.br; derme@unb.br

The knowledge of Paleozoic ostracods from Brazil is scarce and patchy, consisting only of isolated mentions. The first Ordovician ostracods, Satiellina paranaensis Adorno and Salas, and Conchoprimitia brasiliensis Adorno and Salas were recently recorded in the Paraná basin. Currently, new material allowed the identification of Harpabollia n. sp. The species come from the lower levels of the Vila Maria Formation (Upper Ordovician-Silurian), composed of black shales at its base and sandstone to the top. The mudstone interval was interpreted as a periglacial marine environment from mid to outer continental shelf. This unit and the underlay lapó Formation would register the Hirnantian glaciation, the periglacial environment and its subsequent recovery. This sequence and the recorded faunas show a correlation between the Brazilian and North African basins. The presence of Harpabollia and Satiellina support the connection between these basins together with the periGondwanic platforms (Iberia, Armorica, Carnic Alps) and Bohemia. The first record of Harpabollia occurs in Sandbian levels of the Argentinean Precordillera and younger records occur in North Gondwana and Peri-Gondwana during the Late Ordovician. The Harpabollia harparum association well studied and known in Carnic Alps, Bohemia and Baltoscandian Palaeobasin, is considered part of the Hirnantia fauna representing a cold-water assemblage. The presence of Harpabollia in the Paraná basin is consistent with this distribution, however, a deeper analysis and the record of more material is necessary for a better knowledge of the studied fauna.

\section{PALINOLOGÍA DE LA FORMACIÓN MATA AMARILLA, CRETÁCICO SUPERIOR DE LA CUENCA AUSTRAL: CORRELACIONES Y EDAD}

\author{
P.E. Santamarina ${ }^{1}$, V.D. Barreda ${ }^{1}$, A. Iglesias ${ }^{2}$ y A.N. Varela ${ }^{3}$
}

${ }^{1}$ Museo Argentino de Ciencias Naturales "Bernardino Rivadavia" (MACN)-Consejo Nacional de Investigaciones Científicas y Tecnológicas (CONICET). Av. Ángel Gallardo 470, C1405DJR Ciudad Autónoma de Buenos Aires, Argentina. santamarinape@gmail.com; vbarreda@macn.gov.ar ${ }^{2}$ Instituto de Investigaciones en Biodiversidad y Medioambiente (INIBIOMA), Consejo Nacional de Investigaciones Científicas y TecnológicasUniversidad Nacional del Comahue (CONICET-UNCO). Quintral 1250, R8400FRF San Carlos de Bariloche, Río Negro, Argentina. ari_iglesias@yahoo.com.ar

${ }^{3}$ Centro de Investigaciones Geológicas (CIG), Universidad Nacional de La Plata-Consejo Nacional de Investigaciones Científicas y Tecnológicas (CONICET). Diagonal 113 n²74, B1904DPK La Plata, Buenos Aires, Argentina. augustovarela@cig.museo.unlp.edu.ar

La edad de los sedimentos cretácicos continentales aflorantes en los alrededores de la ciudad de Tres Lagos, provincia de Santa Cruz, es controvertida. En la actualidad y en base de datación con circones, existen dos posturas: que fueron depositados durante el Cenomaniano-Turoniano, o que pertenecen al intervalo Campaniano-Maastrichtiano y se correlacionarían con la Formación Cerro Fortaleza. En la presente contribución se expone el resultado del análisis palinológico de la Formación Mata Amarilla, obtenido de dos localidades cercanas a la ciudad de Tres Lagos. Se realizó el conteo de granos para analizar la estructura general de la asociación y sus cambios a lo largo del tiempo. Como resultado, se describe una baja abundancia de granos de angiospermas, junto a una alta diversidad y gran abundancia de esporas de helechos y granos de gimnospermas, posicionando a la asociación en comienzos del Estadio III para Patagonia (Albiano-Cenomaniano). La elevada representación de ciertas especies de Cheirolepidiaceas y Araucariáceas caracteriza a la Subprovincia Classopollis/ Cyclusphaera psilata de edad albiana-cenomaniana. A su vez, la presencia de las megasporas Arcellites disciformis y Balmeisporites cf. holodictyus puede ser referida a la biozona M3 de edad Albiano tardío-Cenomaniano. Por último, no se lograron identificar granos de polen de Proteaceae y Nothofagaceae, elementos comunes en las palinofloras de edad maastrichtiana (Provincia Proteacidites/Nothofagidites) en Patagonia. Desde el punto de vista palinoestratigráfico se concluye que la edad cenomaniana dada por circones para la sección media de la Formación Mata Amarilla es concordante, situándose estratigráficamente muy por debajo de la Formación Cerro Fortaleza. 


\title{
DOS NUEVOS GÉNEROS DE LA TRIBU CHLAMYDINI TEPPNER (PECTINIDAE, BIVALVIA) DEL NEÓGENO DEL EXTREMO AUSTRAL DE AMÉRICA DEL SUR
}

\author{
M.B. Santelli ${ }^{1}$, C.J. Del Río ${ }^{1}$ y M.J. Alvarez ${ }^{1 *}$ \\ 'Museo Argentino de Ciencias Naturales "Bernardino Rivadavia". Av. Ángel Gallardo 470, C1405DJR Ciudad Autónoma de Buenos Aires, Argentina. \\ mbsantelli@gmail.com; claudiajdelrio@gmail.com; maxialvarez82@gmail.com
}

Las especies chilenas de Chlamydini son comunes en los afloramientos neógenos del norte a centro de Chile. Tradicionalmente han sido asignadas a los géneros Chlamys Röding (Plioceno-Reciente, Hemisferio Norte) y Zygochlamys Ihering (Oligoceno Tardío-Mioceno Medio, Patagonia), aunque poseen marcadas diferencias con los representantes de estos, tal como la presencia de conchillas subcirculares o flabeliformes, opistoclinas, con escotadura bisal más somera, y a diferencia de Zygochlamys poseen una ornamentación más uniforme y de presentar conchillas plegadas, los pliegues no son pareados. De esta manera se propone agrupar en un nuevo género endémico del Mioceno Medio-Plioceno medio de Chile a dos de ellas, Z. hupeanus Philippi y Pecten remondi Philippi (= P. coquimbensis Möricke), caracterizado por conchillas grandes, tan altas como largas, ornamentadas por pliegues homogéneos con costillas primarias y secundarias que surgen a partir de los $40 \mathrm{~mm}$, con aurículas levemente asimétricas, y aurículas posteriores con margen libre convexo o recto. La presencia de conchillas subcirculares, ornamentadas por costillas simples cubiertas por escamas bajas muy espaciadas y separadas por interespacios angostos permiten ubicar a C. calderensis Möricke junto a una nueva especie muy poco abundante de la Formación Camarones (Argentina) en otro nuevo género endémico del Mioceno Medio temprano-Plioceno distribuido en el extremo austral de América del Sur. Además, se destaca que el género circumpolar Psychrochlamys Jonkers no está relacionado a estas especies, rechazando la propuesta previa que vinculaba a $Z$. hupeanus con la especie tipo de Psychrochlamys.

*Proyecto financiado por PIP 1649 y PIP 320, PICT -ANPCyT 57.

\section{INTO ANABLEPID DIVERSIFICATION: TWO NEW LATE MIOCENE TAXA (TELEOSTEI: CYPRINODON- TIFORMES) FROM NORTHWESTERN ARGENTINA}

\author{
E. Sferco ${ }^{1}$, G. Aguilera ${ }^{2}$ y J.M. Mirande ${ }^{2 *}$ \\ ${ }^{1}$ Centro de investigaciones en Ciencias de la Tierra (CICTERRA)-Consejo Nacional de Investigaciones Científicas y Técnicas (CONICET), Uni- \\ versidad Nacional de Córdoba. Av. Vélez Sársfield 1611, X5016CGA Córdoba, Córdoba, Argentina. \\ ${ }^{2}$ Unidad Ejecutora Lillo, Consejo Nacional de Investigaciones Científicas y Técnicas (CONICET)-Fundación Miguel Lillo. Miguel Lillo 251, \\ T4000JFE San Miguel de Tucumán, Tucumán, Argentina.aguileragaston@gmail.com; mcmirande@gmail.com
}

At present, the family Anablepidae is composed of three genera: Jenynsia Günther, Anableps Linnaeus and Oxyzygonectes Fowler, which live in brackish and freshwater habitats from Mexico to southern South America. In northern and central Argentina, several species of onesided livebearers (Jenynsia) are important components of freshwater environments. The family is particularly interesting as Jenynsia and Anableps are viviparous fish with sexual dimorphism, mainly exhibited by the modification of the male anal-fin into a gonopodium. Anablepid fossil record includes a premaxilla of Jenynsia sp. from the Pleistocene and two new fossil species described for the Middle Miocene Río Sali Formation of Argentina: Tucmanableps cionei, and Sachajenynsia pacha Sferco, Herbst, Aguilera and Mirande. We present here two new fossil anablepids from the Late Miocene San José Formation of Catamarca and Tucumán provinces that are different from all other known species of the family. One of them represents a new genus and species showing an incipient gonopodium formed by all anal-fin rays. In the performed phylogenetic analysis, and regarding the position of its dorsal-fin, this taxon is recovered as basal to all remaining anablepids with a well-differentiated gonopodium. The other taxon represents the first fossil species of Jenynsia and exhibits a gonopodium formed by anal-fin rays 3,5-7. Because of the inclination of proximal male anal-fin radials and the segmentation present in the sixth ray of the fin, it is recovered within the subgenus Jenynsia in our cladistic hypothesis. These new results suggest the diversity of anablepids was greater in the past.

*Financial support by PICT 2016-1263. 


\title{
REVISIÓN DE LOS MESOTHERIINAE (NOTOUNGULATA) DE LA FORMACIÓN CERRO AZUL (MIOCENO TARDÍO), LA PAMPA, ARGENTINA
}

\author{
R. Sostillo ${ }^{1}$, M.C. Cardonatto ${ }^{1}$, E. Cerdeño ${ }^{2}$ y C.I. Montalvo ${ }^{1 *}$
}

${ }^{1}$ Facultad de Ciencias Exactas y Naturales, Universidad Nacional de La Pampa, Uruguay 151, L6300CLB Santa Rosa, La Pampa, Argentina. renata.sostillo@gmail.com; mccardonatto@gmail.com; cmontalvolp@yahoo.com.ar

2Instituto Argentino de Nivología, Glaciología y Ciencias Ambientales (IANIGLA), Centro Científico Tecnológico Mendoza-Consejo Nacional de Investigaciones Científicas y Tecnológicas (CONICET). Avenida Ruiz Leal s/n, 5500 Mendoza, Mendoza, Argentina. espe@mendoza-conicet.gob.ar

Se analizaron restos asignados a la Subfamilia Mesotheriinae (Notoungulata) recuperados de 26 localidades de la Formación Cerro Azul (Mioceno Tardío) en la provincia de La Pampa. Para evaluar su representación se calculó el número mínimo de individuos (NMI) en las localidades que aportaron la mayor abundancia de mamíferos, resultando entre el 7,7\% y el 25,9\% del total del NMI de macromamíferos, lo que indica que se trata de un grupo frecuente en estas faunas. Typotheriopsis silveyrai Cabrera y Pseudotypotherium subinsigne Rovereto son las especies reconocidas para el Huayqueriense del centro de Argentina. Pseudotypotherium se caracterizaba por la presencia de un surco lingual en el P4, el i1 de sección trapezoidal y los molariformes superiores más imbricados que en Typotheriopsis. Recientemente, se han propuesto nuevos caracteres para diferenciar ambos géneros, muchos de los cuales facilitaron la determinación de algunos ejemplares de la muestra estudiada hasta el nivel de especie. También se identificaron especímenes de tamaño pequeño, atribuidos a individuos juveniles de estos dos géneros. Por otra parte, en varias localidades se reconoció la presencia de Typotheriopsis chasicoensis Cabrera y Kraglievich, especie típica de la edad Chasiquense, de tamaño menor que los taxones huayquerienses. Su registro en Cerro La Bota y Cerro Patagua avala la ubicación biocronológica de sus respectivas faunas en esa edad, mientras que su identificación en Telén y Algarrobo del Águila, cuyas faunas son las más antiguas dentro del Huayqueriense, sugiere la persistencia de T. chasicoensis en esta edad.

*Proyecto financiado por 06-G, Facultad de Ciencias Exactas y Naturales, UNLPam.

\section{COLECCIÓN PALEONTOLÓGICA DEL MUSEO PALEONTOLÓGICO EGIDIO FERUGLIO (MEF): CRECIMIENTO, ESTADO ACTUAL Y DESAFÍOS FUTUROS}

\author{
J. Sterli ${ }^{1}$, J.L. Carballido ${ }^{1}$, I. Escapa ${ }^{1}$, M.A. Pagani ${ }^{1}$, M.E. Pérez ${ }^{1}$, L. Reiner ${ }^{1}$ y E. Ruigómez ${ }^{1}$
}

${ }^{1}$ Consejo Nacional de Investigaciones Científicas y Técnicas (CONICET). Museo Paleontológico Egidio Feruglio. Av. Fontana 140, U9100GYO Trelew, Chubut,Argentina.jsterli@mef.org.ar; jcarballido@mef.org.ar; iescapa@mef.org.ar; apagani@mef.org.ar; mperez@mef.org.ar; Ireiner@mef.org.ar; eruigomez@mef.org.ar

La colección paleontológica del MEF experimentó un crecimiento continuo desde sus comienzos, en 1990 hasta la actualidad, el cual se proyecta de la misma manera para el futuro. En la actualidad el MEF cuenta con más de 30.000 piezas paleontológicas (incluyendo 226 materiales tipo) que se encuentran divididas en tres colecciones: Paleobotánica y Palinología, Paleontología de Vertebrados y Paleontología de Invertebrados e Icnología. El crecimiento de la colección fue de la mano del incremento de los recursos humanos vinculados a la investigación que se han ido incorporando al MEF durante los últimos 10 años, y a intensas colaboraciones con investigadores de otras instituciones del país y del extranjero desde los comienzos de la Institución. Este crecimiento conllevó la necesidad de desarrollar un nuevo esquema organizativo de la colección con la conformación de un Consejo de Curadores y la incorporación de un técnico y voluntarios. Para responder a los desafíos del desarrollo de la colección, los recursos humanos en investigación, y las interacciones con otros investigadores y otras instituciones (e.g., visitas a colección, pedidos de préstamo de materiales), se desarrolló un protocolo de manejo de la colección, mediante el cual se establecen los mecanismos de utilización de la colección por parte de aquellas personas que así lo requieran. Los desafíos futuros se relacionan a: incrementar la digitalización de la colección, tener una llegada más global a través de plataformas globales de datos (e.g., iDigBio) e incorporar otros tipos de colecciones (e.g., actuales, ADN). 


\title{
NEW RECORDS OF MARINE TURTLES (TESTUDINES: CHELONIOIDEA) FROM THE MIOCENE OF CHUBUT, ARGENTINA
}

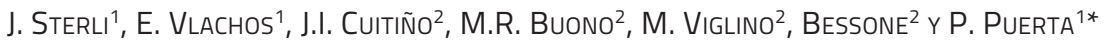 \\ ${ }^{1}$ Consejo Nacional de Investigaciones Científicas y Técnicas (CONICET), Museo Paleontológico Egidio Feruglio, Av. Fontana 140, U9100GYO, \\ Trelew, Chubut, Argentina.jsterli@mef.org.ar; evlacho@mef.org.ar; ppuerta@mef.org.ar \\ 2Instituto Patagónico de Geología y Paleontología (IPGP), Centro Nacional Patagónico (CENPAT)-Consejo Nacional de Investigaciones Cien- \\ tíficas y Tecnológicas (CONICET). Boulevard Brown 2915, U9120ACD Puerto Madryn, Chubut, Argentina. jcuitino@cenpatconicet.gob.ar; \\ buono@cenpat-conicet.gob.ar; viglino@cenpat-conicet.gob.ar; bessone@cenpat-conicet.gob.ar
}

Extinct turtles associated to marine environments in Argentina have a discontinuous record since the Late Jurassic to the Miocene. These turtles adapted to marine environments belong to different clades: the extinct Platychelyidae (PanPleurodira) and Thalassochelyidia (Pan-Cryptodira), and the extant Chelonioidea (formed by two clades: Dermochelyidae and Cheloniidae). The study of fossil marine turtles has its own challenges, as the cosmopolitan distribution of the extant taxa, and probably the extinct as well, makes any fossil of marine turtles relevant beyond its regional context. Up to now, only fragmentary and undetermined specimens of dermochelyids were known for the Miocene marine deposits of Chubut. Recent fieldworks leaded by researchers from CENPAT have brought to light an almost complete shell with some postcranial elements of a dermochelyid turtle from Estancia La Redonda Chica (near Trelew). Also, some fragmentary remains were collected in Bryn Gwyn Geopark (near Gaiman). A partial shell and postcranial remains from Punta Buenos Aires and a partial skull (Península Valdés), and a beak (Bryn Gwyn Geopark) are the first records of pan-cheloniids in Chubut. All the specimens were unearthed from Miocene shallow shelf deposits of the Gaiman Formation (Early Miocene) and Puerto Madryn Formation (Late Miocene). These findings are promising, as they will provide valuable information on the anatomy, taxonomy and diversity of the marine turtles that once populated the seas and coasts of Chubut.

*Financial support by PICT 2015-0792; SAREM; Society Marine Mammalogy; Cetacean Society International.

\section{BRYOZOA DEL PÉRMICO INFERIOR DE PATAGONIA (CUENCA TEPUEL-GENOA, CHUBUT)}

\author{
C.A. Taboada ${ }^{1}$, M.A. Pagani ${ }^{2}$, A.C. Taboada ${ }^{3}$ y M.G. Carrera ${ }^{4}$ \\ ${ }^{1}$ Facultad de Ciencias Naturales y Ciencias de la Salud-Sede Trelew, Universidad Nacional de la Patagonia San Juan Bosco. 9 de Julio y Belgrano, \\ 9100 Trelew, Chubut, Argentina. tukunaturales@gmail.com \\ 2Museo Paleontológico Egidio Feruglio. Avenida Fontana 140, U9100GY0 Trelew, Chubut, Argentina. apagani@mef.org.ar \\ ${ }^{3}$ Centro de Investigación Esquel de Montaña y Estepa Patagónica (CIEMEP). Roca 780, U9200CIL Esquel, Chubut, Argentina. \\ ataboada@unpata.com.ar \\ ${ }^{4}$ Centro de Investigaciones en Ciencias de la Tierra (CICTERRA). Avenida Vélez Sarfield 299, 5016CGA Córdoba Capital, Córdoba, Argentina. \\ mcarrera@com.uncor.edu
}

Los Bryozoa constituyen uno de los grupos mejor representados en las faunas del Paleozoico superior de Patagonia, junto con braquiópodos, bivalvos y gastrópodos, entre los más abundantes. Los briozoos son especialmente conocidos en afloramientos carboníferos de la cuenca, siendo su conocimiento muy escaso en estratos más jóvenes, con solo dos especies pérmicas correspondientes a la Formación Río Genoa. Nuevos estudios realizados en la Formación Mojón de Hierro, en el área de El Molle, han revelado una mayor diversidad del grupo en la región. Se ha identificado Septatopora sp., Rectifenestella sp. aff. R. columnaris Crockford y Fenestellidae gen. et. spp. indet. (Orden Fenestrata), además de Glyptopora sp. y Prismopora sp. aff. P. digitata Crockford (Orden Cystoporata) y Rhabdomeson sp. aff. R. mamillatum Bretnall (Orden Cryptostomata), constituyendo una asociación indicadora de un ambiente marino somero bajo un régimen energético moderado y un sustrato blando a suficientemente firme (onshore). La presencia en Patagonia de Glyptopora, Prismopora, y Rhabdomeson amplía su distribución geográfica al margen sudoccidental de Gondwana. Los taxones $R$. aff. columnaris, $P$. aff. digitata y $R$. aff. mamillatum muestran afinidades con especies del Pérmico inferior (Artinskiano) de las cuencas Canning y Carnarvon, reforzando la vinculación paleobiogeográfica entre Patagonia y el oeste de Australia durante el Cisuraliano. Dicho vínculo, al cual también se suma Septatopora, indica que durante el Pérmico Temprano Patagonia formó parte del Dominio Gondwánico, pertenencia ignorada en las más recientes reconstrucciones paleobiogeográficas en base a briozoos, sostenida en contraparte por faunas de braquiópodos asociadas. 


\title{
PRIMER REGISTRO DE CONOPEUM GRAY (ELECTRIDAE, BRYOZOA) EN EL CRETÁCICO SUPERIOR DE LA FORMACIÓN LEFIPÁN (CHUBUT, PATAGONIA)
}

\author{
C.A. TABOAda ${ }^{1}$, M.A. Pagani² Y R. CÚneO²
}

${ }^{1}$ Facultad de Ciencias Naturales y Ciencias de la Salud-Sede Trelew, Universidad Nacional de la Patagonia San Juan Bosco. 9 de Julio y Belgrano, 9100 Trelew, Chubut, Argentina. tukunaturales@gmail.com

2Museo Paleontológico Egidio Feruglio. Av. Fontana 140, U9100GYo Trelew, Chubut, Argentina. apagani@mef.org.ar; rcuneo@mef.org.ar

La radiación adaptativa del Orden Cheilostomata (Bryozoa) durante el Cretácico Tardío es de particular interés debido a la rápida expansión y evolución explosiva de innovaciones morfológicas que aparecieron en el grupo. Sin embargo, el conocimiento de briozoos cretácicos es muy escaso globalmente, especialmente en Sudamérica y particularmente en Argentina. En la Formación Lefipán se ha reconocido una fauna monoespecífica de briozoos epifíticos sobre hojas de angiospermos fósiles. La Formación Lefipán es una unidad siliciclástica del Maastrichtiano-Daniano en el ámbito NO de la Cuenca Cañadón Asfalto, la cual completa el ciclo de relleno del rift iniciado a principios del Jurásico. Sus condiciones de sedimentación corresponden a depósitos litorales representados por plataformas someras y deltas dominados por mareas. Asimismo se registra en la unidad la transición $\mathrm{K} / \mathrm{Pg}$, aunque en un contexto dinámico afectado por la fase marina transgresiva iniciada a fines del Maastrichtiano. Los ejemplares estudiados corresponden a una nueva especie de queilostomado membraniporiforme asignable al género Conopeum. Briozoos modernos de hábitos de crecimiento membraniporiformes son componentes comunes de ambientes marinos de plataforma somera, intermareal a supramareal con niveles de energía moderada a alta y sustratos duros a flexibles. En la actualidad también se registran formas epifíticas de briozoos caracterizadas por ser fotófilos y por habitar ambientes salobres como albuferas, estuarios y deltas presentando hábitos eurihalinos y adaptados a la naturaleza efímera y flexible del sustrato. Tales condiciones se verifican en la Formación Lefipán, por lo que los briozoos identificados confirman interpretaciones previas.

\section{NECROSIS ÓSEA EN UN EJEMPLAR JUVENIL DE MOSASAURIO DE ANTÁRTIDA}

\author{
M. Talevil ${ }^{1}$, M. FernándezZ², M. Mitidieri ${ }^{3}$ Y M. Reguero $2,4 *$ \\ ${ }^{1}$ Instituto de Investigación en Paleobiología y Geología (IIPG)-Consejo Nacional de Investigaciones Científicas y Tecnológicas (CONICET), \\ Universidad Nacional de Río Negro. Av. Roca 1242, R8332EXZ Gral. Roca, Río Negro, Argentina. mtalevi@unrn.edu.ar \\ ${ }^{2}$ División Paleontología de Vertebrados, Museo de La Plata, Facultad de Ciencias Naturales y Museo, Universidad Nacional de La Plata. Paseo \\ del Bosque s/n, B1900FWA La Plata, Buenos Aires, Argentina. martafer@fcnym.unlp.edu.ar \\ 3Universidad Nacional de Río Negro. Estados Unidos 750, R8332BRJ General Roca, Río Negro, Argentina.matiasmitidieri@yahoo.com.ar \\ 4Instituto Antártico Argentino. 25 de Mayo 1151, B1650HMK San Martín, Buenos Aires, Argentina. regui@fcnym.unlp.edu.ar
}

El reconocimiento y estudio de ciertas patologías óseas en el registro fósil, ha permitido conocer aspectos precisos de la fisiología y paleoecología de algunos grupos de reptiles marinos. A partir del análisis de las características osteológicas y microanatómicas se pueden determinar las limitaciones resultantes que pudieron afectar el comportamiento del animal. En el presente trabajo se estudia una escápula izquierda de un mosasaurio juvenil recuperado de niveles maastrichtianos (Cretácico Superior) de la Formación López de Bertodano, Isla Marambio, Antártida. La muestra se analizó combinando la observación macroscópica y el uso de la microtomografía axial computarizada ( $\mu$-TAC), la cual permitió observar la estructura interna en tres dimensiones y la extensión de la condición patológica, así como la conservación de la muestra. En el elemento analizado se observa el colapso del tejido óseo (necrosis ósea) en la cara interna del cuello escapular por encima de la faceta glenoidea. Las características morfológicas de esta patología son compatibles con las de un proceso infeccioso. Asimismo, los tejidos sanos circundantes indican que el individuo no continuó creciendo con posterioridad a aparición de la lesión y permiten suponer que la muerte del animal fue consecuencia de la misma. Un mosasaurio juvenil con esta condición habría tenido dificultades en su locomoción, en la obtención de alimento y lo convertiría eventualmente en una presa fácil.

*Proyecto financiado por Universidad Nacional de Río Negro, PI UNRN 40-A-660; Agencia de Promoción Científica y Tecnológica (ANCyT) PICT 2016-1039 у PICT 2016-0607. 


\title{
PUESTA EN VALOR DEL PATRIMONIO HISTÓRICO DE LA FCEN-UBA. DIAPOSITIVAS SOBRE VIDRIO
}

\author{
M.A. TANuZ ${ }^{1}$ y B. Aguirre-URreta ${ }^{2}$
}

'Departamento de Geología, Facultad de Ciencias Exactas y Naturales, Universidad de Buenos Aires. Intendente Güiraldes 160, Ciudad Universitaria, C1428EGA Ciudad Autónoma de Buenos Aires, Argentina.mtanuz@gl.fcen.uba.ar

2Departamento de Geología, Facultad de Ciencias Exactas y Naturales, Instituto de Estudios Andinos "Don Pablo Groeber" (IDEAN), Universidad de Buenos Aires-Consejo Nacional de Investigaciones Científicas y Tecnológicas (CONICET). Intendente Güiraldes 2160, Ciudad Universitaria - Pabellón II, C1428EGA Ciudad Autónoma de Buenos Aires, Argentina. aguirre@gl.fcen.uba.ar

Hacia fines del siglo XIX y durante la primera mitad del siglo XX las diapositivas sobre vidrio eran el principal apoyo visual para ilustrar el dictado de clases y conferencias en la Universidad de Buenos Aires. Las diapositivas constan de dos placas de vidrio unidas entre sí en cuyo interior yace una imagen que fue expuesta y procesada. Para su proyección en las aulas se utilizaban los epidiascopios, aparatos capaces de amplificar la imagen de cuerpos opacos y de las diapositivas sobre vidrio a través del uso de una lámpara y un juego de lentes y espejos. Estos materiales forman parte del patrimonio histórico de la enseñanza en la Universidad de Buenos Aires. El objetivo de este trabajo es presentar una iniciativa en materia de conservación para la puesta en valor de las más de 300 diapositivas sobre vidrio pertenecientes al Área de Paleontología del Departamento de Geología de la FCEN-UBA. Se realizará, siguiendo los estándares internacionales en la materia, la identificación, organización, evaluación del estado de conservación, elección de un ambiente de almacenamiento, y la digitalización de cada diapositiva. Cabe destacar que la exposición de algunas de estas diapositivas en el marco de la Noche de los Museos 2017, despertó en los visitantes interés y admiración debido a que desconocian su existencia y la calidad de sus imágenes. Esto fortalece nuestra convicción que solo se puede valorar aquello que se conoce y reafirma nuestro compromiso de procurar las mejores condiciones curatoriales para que esto sea posible.

\section{EARLY OLIGOCENE FORAMINIFERAL AND CALCAREOUS NANNOFOSSIL ASSEMBLAGE FROM THE SOUTHERN AUSTRAL BASIN, TIERRA DEL FUEGO, SOUTH AMERICA}

\author{
J.M. Thissen ${ }^{1}$, And J.P. Pérez Panera ${ }^{1 *}$ \\ 1YPF Tecnología. Av. del Petróleo Argentino s/n (entre 129 y 143), 1923, Berisso, Buenos Aires, Argentina. jens.m.thissen@ypftecnologia.com; \\ juan.p.panera@yptecnologia.com
}

The Austral basin is one of the most prolific depositional environments in the southernmost part of the Americas and is of interest for hydrocarbon exploration in this area. Due to its location, the basin is important for the understanding of the orogeny of the southern and Fuegian Andes as well as the evolution of high-latitude calcareous microfossils. In this contribution, we characterize an Early Oligocene foraminiferal and calcareous nannofossil assemblage from the subsurface Chilean sector of the Isla Grande de Tierra del Fuego. Foraminifera are characterized by a low diversity of benthic forms like Globobulimina pacifica, Sphaeroidina bulloides, Protoglobobulimina pupoides, and several agglutinated taxa. Planktic forams are badly preserved specimens of the genera Globigerina, Globorotalia, and Globigerinoides. Only Globoturborotalita labiacrassata was found to be an identifiable index species for the Oligocene. The foraminifera are accompanied by a high number of radiolarians which indicates open water conditions. The calcareous nannofossil assemblage is of low diversity, poorly preserved, but abundant. It is dominated by species of the genus Reticulofenestra, and most abundant are Reticulofenestra daviesii, R. minuta, R. bisecta and R. filewiczii. Other important taxa are Coccolithus pelagicus, Cyclicargolithus floridanus and Chiasmolithus altus, the latter could reach high abundance in some intervals. This assemblage is characteristic of cold surface waters.

*Financial support by Y-TEC I+D 602; CONICET PDT S 153. 


\title{
COPROLITOS ASIGNABLES A SPARASSODONTA (METATHERIA) DEL MIOCENO TEMPRANO DE PATAGONIA, ARGENTINA: ASPECTOS PALEOBIOLÓGICOS Y PALEOECOLÓGICOS
}

\author{
R.L. Tomassini ${ }^{1}$, C.I. Montalvo², M.S. Bargo ${ }^{3}$ y S.F. VizCaíno ${ }^{3 *}$ \\ 'Departamento de Geología, Universidad Nacional del Sur (UNS)-Instituto Geológico del Sur (INGEOSUR)-Consejo Nacional de Investigaciones Cien- \\ tíficas y Tecnológicas (CONICET). Av. Alem 1253 cuerpo B' $1^{\circ}$ Piso, B8000CPB Bahía Blanca, Buenos Aires, Argentina. rodrigo.tomassini@yahoo.com.ar \\ ${ }^{2}$ Facultad de Ciencias Exactas y Naturales, Universidad Nacional de La Pampa, Uruguay 151, L6300CLB Santa Rosa, La Pampa, Argentina. \\ cmontalvo@exactas.unlpam.edu.ar \\ ${ }^{3}$ División Paleontología de Vertebrados, Unidades de Investigación Anexo Museo, Facultad de Ciencias Naturales y Museo, Universidad Nacional \\ de La Plata. Av. 60 y 122 s/n, B1904 La Plata, Buenos Aires, Argentina.msbargo@fcnym.unlp.edu.ar; vizcaino@fcnym.un/p.edu.ar
}

La presencia de coprolitos en el registro fósil brinda información para estudios paleobiológicos y paleoecológicos. Se describe una muestra de 112 coprolitos procedentes de depósitos fluviales de la Formación Santa Cruz (Mioceno inferior), aflorantes en distintas localidades de la provincia de Santa Cruz. Se interpretan sus posibles productores e interacciones tróficas en este paleoecosistema. Se evaluó tamaño (largo, ancho, espesor), forma (relación largo/ancho vs ancho/espesor), composición mineralógica y características superficiales de los coprolitos. Se diferenciaron dos morfotipos según sus dimensiones y morfologías. Los ejemplares del morfotipo I son ovoides y tienen 20 mm de largo promedio; los del morfotipo II son cilíndricos y tienen $\sim 27 \mathrm{~mm}$ de largo promedio. En ambos se registró fluorapatita e hidroxiapatita, componentes característicos de coprolitos de carnívoros. Además, presentan extremos en punta, alta porosidad, constricciones y trazas de pelos. El 40\% presenta inclusiones óseas, incluyendo astillas indeterminables y elementos esqueléticos de roedores Octodontoidea y Chinchilloidea. Estos restos mostraron modificaciones producidas por digestión: redondeamiento de bordes, agrietamiento del tejido cortical y exposición del tejido esponjoso. Se identificaron perforaciones y surcos asignables a coprofagia, lo que refleja que las heces estuvieron expuestas un tiempo en superficie y fueron aprovechadas por invertebrados. Aun cuando no puede identificarse con precisión el productor, la fauna conocida para la Formación Santa Cruz, las dimensiones de los coprolitos y los restos óseos recuperados, permiten vincular ambos morfotipos con metaterios Sparassodonta, posiblemente Hathliacynidae y/o pequeños Borhyaenidae. Las evidencias sugieren, además, que la dieta de estos carnívoros habría estado basada fundamentalmente en pequeños mamíferos.

*Proyecto financiado por PICT 2013-0389; UNLP 11/N750; NGS CP-030R-17.

\section{FIRST CONODONT RECORD IN THE ARGENTINE PUNA RELATED TO MIDDLE ORDOVICIAN GRAPTOLITES}

\author{
B.A. Toro ${ }^{1}$, S. Heredia ${ }^{2}$, N.C. Herrera Sánchez ${ }^{1}$, F. Moreno ${ }^{2}$ y G. Lo Valvo ${ }^{3 *}$
}

${ }^{1}$ Centro de investigaciones en Ciencias de la Tierra (CICTERRA)-Consejo Nacional de Investigaciones Científicas y Técnicas (CONICET) y Universidad Nacional de Córdoba. Av. Vélez Sársfield 1611, X5016CGA Córdoba, Córdoba, Argentina.btorogr@mendoza-conicet.gov.ar; nexxysherrera@gmail.com

2Laboratorio de Micropaleontología, Instituto de Investigaciones Mineras, Universidad Nacional de San Juan-Centro de Investigaciones de la Geósfera y la Biósfera (CIGEOBIO), Consejo Nacional de Investigaciones Científicas y Tecnológicas (CONICET). Libertador General San Martín 1109, J5400ARL San Juan, San Juan, Argentina. sheredia@unsj.edu.ar; florenciamorenovaldez@gmail.com

${ }^{3}$ Facultad de Ciencias Exactas Universidad de Buenos Aires. Int. Güiraldes 2160, C1428EGA Ciudad Autónoma de Buenos Aires, Buenos Aires, Argentina.gerardolovalvo@gmail.com

Newly biostratigraphic research conducted in the Huaytiquina section (Salta province), allowed to obtain the first record of Middle Ordovician conodonts for the western Argentinean Puna. The analyzed material comes from calcareous sandstones levels intercalated in the lower portion of the turbiditic succession corresponding to the Coquena Formation. The recovered conodont fauna is composed by species of the genera Baltoniodus, Gothodus, Trapezognathus, Drepanoistodus, Drepanodus, few elements of Microzarkodina and Protopanderodus, among others. Although the conodont elements are very abundant, the diversity is very low, being the Gothodus elements the most numerous in each sample. The conodont associations indicate a Dapingian (Middle Ordovician) age, linking the conodonts of the Argentinean Puna with those from Baltoscandinavia. The productive levels also contain graptolites of the Tetragraptus genus, and are closely related to strata bearing Azygograptus lapworthi Nicholson and Xiphograptus lofuensis Lee which are confirming the Dapingian age of the lower and middle portions of the Coquena Formation. The occurrence of Azygograptus lapworthi was also recently recorded in the Argentinean Cordillera Oriental related to the index conodont Baltoniodus triangularis Lindström. The new findings, suggest that a high resolution correlation between both geomorphological regions could be possible, and they also certify that both sectors of the Central Andean basin were connecting during the interval corresponding from the Lower Ordovician (Floian) to the Middle Ordovician (Dapingian). Future taxonomic studies of larger conodont collections and graptolites key taxa will make it possible to accurate the age of the Coquena Formation, improving its regional and global correlations. 


\title{
NUEVOS EJEMPLARES DE PROTEROCHÁMPSIDOS INCREMENTAN LA DIVERSIDAD MORFO- LÓGICA DE LOS RHADINOSUQUINOS DE LA FORMACIÓN CHAÑARES (CARNIANO INFERIOR), PROVINCIA DE LA RIOJA, ARGENTINA
}

\author{
M.J. Trotteyn ${ }^{1}$, M. Ezcurra², M.B. Von Baczko ${ }^{3}$, And J. Desojo ${ }^{3 *}$
}

\begin{abstract}
${ }^{1}$ Centro de Investigaciones de la Geósfera y la Biósfera (CIGEOBIO), Consejo Nacional de Investigaciones Científicas y Tecnológicas (CONICET). Instituto de Geología "Emiliano Pedro Aparicio", Departamento de Geología, Facultad de Ciencias Exactas, Físicas y Naturales, Universidad Nacional de San Juan. Av. de la Roza Oeste 590, J5402DCS San Juan, San Juan, Argentina. jtrotteyn@gmail.com

2Sección Paleontología de Vertebrados, Museo Argentino de Ciencias Naturales "Bernardino Rivadavia" (MACN)-Consejo Nacional de Investigaciones Científicas y Tecnológicas (CONICET). Av. Ángel Gallardo 470, C1405DJR Ciudad Autónoma de Buenos Aires, Argentina. martindezcurra@yahoo.com.ar ${ }^{3}$ División Paleontología Vertebrados, Museo de La Plata, Facultad de Ciencias Naturales y Museo, Universidad Nacional de La Plata. Paseo del Bosque s/n, B1900FWA La Plata, Buenos Aires, Argentina.belenvonbaczko@gmail.com; julideso2@gmail.com
\end{abstract}

Los proterochámpsidos constituyen un grupo de tetrápodos fósiles faunívoros, de pequeño a mediano tamaño y posiblemente semi-acuáticos, que se asemejan en rasgos generales a los cocodrilos actuales. Su registro está restringido a América del Sur, en rocas del Triásico Superior aflorantes en el noroeste de Argentina y el sur de Brasil. Los rhadinosuquinos (Chanaresuchus bonapartei, Gualosuchus reigi, Rhadinosuchus gracilis y Pseudochampsa ischigualastensis) son las formas más derivadas del grupo y constituyen los diápsidos más abundantes en la Zona de Asociación (ZA) de Massetognathus-Chanaresuchus de la Formación Chañares (Carniano inferior; Cuenca de Ischigualasto-Villa Unión), provincia de La Rioja, Argentina. Aquí se describen dos nuevos especímenes de rhadinosuquinos recientemente colectados en esta ZA, los cuales están constituidos por un esqueleto parcial articulado (CRILAR-Pv 491) y elementos craneanos y poscraneanos desarticulados (CRILAR-Pv 488). Este último ejemplar es el más incompleto, pero su buena preservación permite mejorar el conocimiento anatómico de ciertas regiones del esqueleto de este clado (escápula, segunda costilla sacra, miembro posterior). Las combinaciones de estados de caracteres presentes en CRILAR-Pv 488 y CRILAR-Pv 491 incrementan notablemente la diversidad morfológica del grupo y constituyen un mosaico de rasgos considerados previamente diagnósticos de diferentes especies de rhadinosuquinos (e.g., fosa antorbitaria sobre el proceso horizontal del maxilar y lacrimal, osteodermos con forma de X). Como resultado se concluyó que la taxonomía de los especímenes de rhadinosuquinos actualmente conocidos debería ser revisada en detalle, incluyendo una re-evaluación de la variación intraespecífica, ontogenética y tafonómica que afectó a los mismos de diferentes maneras.

*Proyecto financiado por PICT 2016-0481; CICIT CA 2018-2019; PICT 2014-609.

\section{LOS NIVELES MAASTRICHTIANOS NO-MARINOS DE LA FORMACIÓN LAGO COLHUÉ HUAPI: UN PALEOAMBIENTE ACUÁTICO PRÓXIMO AL LÍMITE K/PG EN LA CUENCA DEL GOLFO SAN JORGE}

\author{
P. Vallati ${ }^{1}$, A. De Sosa Tomas $^{1}$ y G.A. Casal ${ }^{2 *}$
}

${ }^{1}$ Laboratorio de Bioestratigrafía, Facultad de Ciencias Naturales y Ciencias de la Salud, Universidad Nacional de la Patagonia San Juan Bosco. Km 4, Ciudad Universitaria, 9000 Comodoro Rivadavia, Chubut, Argentina.patricia.vallati@gmail.com; adesosatomas@gmail.com 2Laboratorio de Paleontología de Vertebrados, Facultad de Ciencias Naturales y Ciencias de la Salud, Universidad Nacional de la Patagonia San Juan Bosco. Km 4, Ciudad Universitaria, 9000 Comodoro Rivadavia, Chubut, Argentina. paleogac@gmail.com

En los niveles maastrichtianos de la Formación Lago Colhué Huapi, en el Cerro del Hadro, nacientes del Río Chico, se han reportado asociaciones palinológicas y fragmentos mesoscópicos de charcoal que permiten realizar interpretaciones paleoambientales. Las pelitas laminadas estudiadas incluyen una asociación de palinomorfos que indican un cuerpo de agua dulce templada y de baja energía con especies marcadoras del Maastrichtiano. Por encima de estas, se encuentran rocas margosas blanquecinas en las que se han registrado esporas de Zygnemataceae, briofitas, licofitas y monilofitas (entre ellas Azolla) que representan la vegetación acuática y las plantas que crecían próximas al cuerpo de agua. Los granos sacados de Podocarpaceae son dominantes en la asociación y las angiospermas incluyen Liliacidites spp. y Spinizonocolpites cf. hialinus. Esta última especie tiene afinidad con las palmeras tipo Nypa que en la actualidad habitan ambientes de manglares costeros, sugiriendo la cercanía del mar Maastrichtiano, como fue propuesto en trabajos anteriores. Asimismo, el nivel de rocas margosas incluye abundante charcoal que indica la ocurrencia de un paleoincendio superficial de baja intensidad. Se reconocen fragmentos de leño de coníferas, con traqueidas con perforaciones areoladas uniseriadas, biseriadas y campos de cruzamiento de morfología variada y probables restos de angiospermas.

*Proyecto financiado por PI 10/C347 "Paleobotánica y Palinología del Cretácico Tardío/Paleoceno en el área del Lago Colhué Huapi y las nacientes del Río Chico". 


\title{
COLECCIÓN RODOLFO F.J. BRUNET: PUESTA EN VALOR DEL PATRIMONIO PALEONTOLÓGICO
}

\author{
J.L. Vallejo ${ }^{1}$, L. Alzugaray², M. Ferrari ${ }^{3}$ y J. Signorelli ${ }^{4}$
}

${ }^{1}$ Facultad de Ciencias Exactas y Naturales, Universidad de Buenos Aires. Intendente Güiraldes 2160, C1428EGA Ciudad Autónoma de Buenos Aires, Argentina. juli.vallejo91@gmail.com

${ }^{2}$ Facultad de Ciencias Naturales, Universidad Nacional de la Patagonia San Juan Bosco. Bvd. Brown 3051, U9120ACE Puerto Madryn, Chubut, Argentina. lualzugaray@hotmail.com

${ }^{3}$ Instituto Patagónico de Geología y Paleontología (IPGP), Centro Nacional Patagónico (CENPAT)-Consejo Nacional de Investigaciones Científicas y Tecnológicas (CONICET). Boulevard Brown 2915, U9120ACD Puerto Madryn, Chubut, Argentina. mferrari@cenpat-conicet.gob.ar ${ }^{4}$ Instituto de Biología de Organismos Marinos (IBIOMAR), Centro Nacional Patagónico (CENPAT)-Consejo Nacional de Investigaciones Científicas y Tecnológicas (CONICET). Boulevard Brown 2915, U9120ACD Puerto Madryn, Chubut, Argentina. jsignorelli@cenpat-conicet.gob.ar

El Instituto Patagónico de Geología y Paleontología (IPGP) cuenta con una colección de Paleontología de Invertebrados e Icnología (CNP-PIIC). Recientemente, más de 3.000 piezas fósiles recolectadas por Rodolfo Brunet han sido donadas al IPGP para ser incorporadas a la colección. En este marco, se desarrolla el proyecto titulado “Manejo y Conservación del Patrimonio Paleontológico: Colección Rodolfo F.J. Brunet" donde se propone realizar una puesta en valor de los ejemplares recolectados por el naturalista aficionado a lo largo de varias décadas. Cumpliendo con lo estipulado por la Ley Nacional 25.743 de Protección del Patrimonio Arqueológico y Paleontológico, se dio inicio a la revisión de la colección en su totalidad mediante trabajos de catalogación y digitalización. Las tareas realizadas incluyen curación de los ejemplares, identificación taxonómica, verificación de procedencia estratigráfica y/o geográfica e ingreso del material para su conservación definitiva en la CNP-PIlc. El material revisado consta principalmente de invertebrados fósiles, siendo la Clase Bivalvia el grupo mejor representado (73\% de la colección-datos preliminares). Ejemplares de la Clase Gastropoda y los phyla Cnidaria, Echinodermata, Bryozoa y Brachiopoda, así como piezas de icnofósiles, microfósiles y artrópodos están presentes en la colección. Se destaca la presencia de material tipo in schedula cuyo status taxonómico es incierto. Los ejemplares provienen de distintas localidades de Patagonia, en su mayoría de la Formación Puerto Madryn (Mioceno Tardío). Un total de 63 lotes han sido ingresados a la CNP-PIlc. Aquellas piezas sin procedencia estratigráfica y/o geográfica y sin valor académico fueron separadas para un uso didáctico y/o divulgativo.

\section{APORTES A LA HISTORIA EVOLUTIVA DE LOS PLATANISTOIDEA DEL MIOCENO TEMPRANO DE PATAGONIA (FORMACIÓN GAIMAN): AONDELPHIS TALEN}

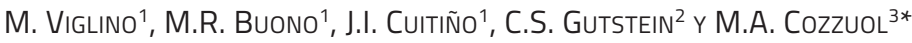 \\ ${ }^{1}$ Instituto Patagónico de Geología y Paleontología (IPGP), Centro Nacional Patagónico (CENPAT)-Consejo Nacional de Investigaciones \\ Científicas y Tecnológicas (CONICET). Boulevard Brown 2915, U9120ACD Puerto Madryn, Chubut, Argentina. viglino@cenpat-conicet.gob.ar; \\ buono@cenpat-conicet.gob.ar; jcuitino@cenpat-conicet.gob.ar \\ ²Red Paleontológica U. Chile, Laboratorio de Ontogenia y Filogenia, Departamento de Biología, Facultad de Ciencias, Universidad de Chile. \\ Las Palmeras 3425,8320000 Santiago, Región Metropolitana, Chile. sgcarolina@gmail.com \\ ${ }^{3}$ Departamento de Zoologia, Instituto de Ciências Biológicas, Universidade Federal de Minas Gerais. Av. Antônio Carlos 6627, 31270-910, \\ Belo Horizonte, Minas Gerais, Brasil.mario.cozzuol@gmail.com
}

El Mioceno Temprano es uno de los momentos más importantes en la historia evolutiva de los cetáceos, sin embargo está escasamente documentado en el registro fósil mundial. Dentro de los odontocetos, la superfamilia Platanistoidea (Oligoceno Tardío/Mioceno Temprano-Actualidad) comprende mayormente especies extintas y una sola actual (Platanista gangetica), si bien el contenido y relaciones filogenéticas dentro de la misma son aún controversiales. El Mioceno Temprano de Patagonia (Formación Gaiman) documenta excepcionalmente la radiación inicial del grupo, con tres especies conocidas: Phoberodon arctirostris, Notocetus vanbenedeni y Prosqualodon australis. En este trabajo se describe y analiza la posición filogenética de un nuevo taxón de Platanistoidea, colectado en la Formación Gaiman. El holotipo (MPEF-PV 517) consiste en un cráneo incompleto con el periótico y bula timpánica asociados. Los análisis anatómicos y filogenéticos permitieron identificarlo como un nuevo género y especie. Dicho taxón se recuperó en una posición basal dentro de los Platanistoidea, formando un clado con un taxón de Nueva Zelanda (ZMT-73). En esta contribución, se clarificó la terminología de la fosa subcircular en el escamoso y se propone una nueva estructura para este hueso (fosa para el proceso articular). Durante el Mioceno Temprano en Patagonia, se identifica la coexistencia de al menos dos morfotipos que no presentarían solapamiento ecológico. Esto permite aumentar la diversidad conocida de platanistoideos para el Mioceno Temprano y apoya la hipótesis de una rápida diversificación durante el origen del mismo en el Hemisferio Sur.

*Proyecto financiado por Cetacean Society International, PICT 2015-0792, Sociedad Argentina para el Estudio de Mamíferos; Smithsonian Instituttion, AMNH, Society for Marine Mammalogy. 


\title{
MASA CORPORAL, TAMAÑO CEREBRAL RELATIVO Y ENCEFALIZACIÓN DE CYONASUA (CAR- NIVORA, PROCYONIDAE): INFERENCIAS PALEOBIOLÓGICAS
}

\author{
M.G. VILCHez BaRRaL ${ }^{1}$, M.T. Dozo ${ }^{1}$ Y L. SOIBeLzon ${ }^{2}$ \\ ${ }^{1}$ Instituto Patagónico de Geología y Paleontología (IPGP), Centro Nacional Patagónico (CENPAT)-Consejo Nacional de Investigaciones Cientí- \\ ficas y Tecnológicas (CONICET). Boulevard Brown 2915, U9120ACD Puerto Madryn, Chubut, Argentina. mguadalupevilchez@cenpat-conicet.gob.ar; \\ dozo@cenpat-conicet.gob.ar \\ 2División Paleontología Vertebrados, Museo de La Plata, Facultad de Ciencias Naturales y Museo, Universidad Nacional de La Plata. Paseo del \\ Bosque s/n, B1900FWA La Plata, Buenos Aires, Argentina. Isoibelzon@fcnym.unlp.edu.ar
}

Los prociónidos son los representantes más antiguos del Orden Carnivora en Sudamérica. Los primeros registros son de Edad/Piso Huayqueriense (Mioceno Tardío) y corresponden al género Cyonasua. Estos carnívoros migraron desde Norteamérica antes que el Istmo de Panamá estuviese completamente formado. En este trabajo se realizaron tomografías axiales computarizadas de cráneos de Cyonasua (MLP 95-III-31-80) y de todos los géneros de prociónidos vivientes. Luego se utilizó software especializado para la reconstrucción de los modelos tridimensionales del cráneo y de la cavidad endocraneana obteniéndose a partir de los modelos, las correspondientes medidas lineales y volúmenes. Con estos datos se hicieron estimaciones de la masa corporal y el tamaño cerebral relativo para Cyonasua. Los valores obtenidos entre 14,6k g y 18,5 kg, indican un mayor tamaño de Cyonasua en relación a los prociónidos vivientes, los cuales tienen masas corporales medias menores a $3 \mathrm{~kg}$. Con respecto al tamaño cerebral relativo, el cociente de encefalización (CE) calculado para Cyonasua (CE= 0,7) fue menor a los obtenidos para los prociónidos vivientes cuyos valores fueron mayores a 1. Dado que el cociente de encefalización explica la relación alométrica entre el tamaño del cerebro y del cuerpo, podría esperarse que el cociente de encefalización sea menor ya que a mayor tamaño corporal menor será el tamaño cerebral relativo. Por otro lado el CE de Cyonasua es consistente con los valores de encefalización obtenidos en carnívoros neógenos de Norteamérica, de tamaño corporal equivalente (Tomarctus, $\mathrm{CE}=0,80$; Cynodesmus, $\mathrm{CE}=0,54$ ).

\section{THE OUTSTANDING RÍO CHALÍA (= SEHUÉN) OUTCROPS OF THE SANTA CRUZ FORMATION (EARLY MIOCENE, BURDIGALIAN) AND ITS FOSSIL VERTEBRATE CONTENT}

\author{
S.F. Vizcaíno ${ }^{1}$, M.S. Bargo ${ }^{1}$, J.I. Cuitiño², M.E. Pérez ${ }^{3}$, N.A. Muñoz ${ }^{1}$, I. Aramendía ${ }^{4}$, R.L. Tomassini ${ }^{5}$, and R.F. Kay ${ }^{6 *}$ \\ 1División Paleontología de Vertebrados, Unidades de Investigación Anexo Museo, Facultad de Ciencias Naturales y Museo, Universidad \\ Nacional de La Plata. Av. 60 y 122 s/n, B1904 La Plata, Buenos Aires, Argentina.vizcaino@fcnym.unlp.edu.ar; msbargo@fcnym.unlp.edu.ar; \\ nahuelmunoz@fcnym.unlp.edu.ar \\ ${ }^{2}$ Instituto Patagónico de Geología y Paleontología (IPGP), Centro Nacional Patagónico (CENPAT)-Consejo Nacional de Investigaciones Cientí- \\ ficas y Tecnológicas (CONICET). Boulevard Brown 2915, U9120ACD Puerto Madryn, Chubut, Argentina. jcuitino@cenpat-conicet.gob.ar \\ 3Museo Paleontológico "Egidio Feruglio". Av. Fontana 140, U9100GY0 Trelew, Chubut, Argentina. mperez@mef.org.ar \\ ${ }^{4}$ Instituto Patagónico para el Estudio de los Ecosistemas Continentales, Centro Nacional Patagónico (CENPAT)-Consejo Nacional de Investigacio- \\ nes Científicas y Tecnológicas (CONICET). Boulevard Brown 2915, U9120ACD Puerto Madryn, Chubut, Argentina. ines.aramendia91@gmail.com \\ 5 Instituto Geológico del Sur (INGEOSUR), Consejo Nacional de Investigaciones Científicas y Tecnológicas (CONICET)-Departamento de Geología, \\ Universidad Nacional del Sur. Av. Alem 1253, B8000CPB Bahía Blanca, Buenos Aires, Argentina. rodrigo.tomassini@yahoo.com.ar \\ ${ }^{6}$ Evolutionary Anthropology and Division of Earth and Ocean Sciences, Duke University. Box 90383, 27708 NC, Durham, North Carolina, \\ Estados Unidos.richard.kay@duke.edu
}

The continental Early Miocene Santa Cruz Formation (SCF) is widely distributed in southern Patagonia. It fossil mammals comprise the basis of the Santacrucian Land Mammal Age. Fossil prospecting of exposures along the southern escarpment of Río Chalía have been neglected until now. They represent the greatest known aerial exposure and sedimentary thickness of all the SCF. The maximum thickness measured in a single locality is $295 \mathrm{~m}$. The SFC in Río Chalía is mostly composed of fine-grained sediments (clay, silt, fine sand) with abundant intermingled pyroclastic material, accumulated in a low-gradient fluvial system. New preliminary U-Pb tuff dates permit us to correlate the Río Chalía SCF levels with those of the Río Santa Cruz and coastal localities. We collected some 480 lots and 10 individual specimens, including fish, frog, squamate, bird and mammal remains. $64 \%$ of the fossils come from horizons near the base of the section (between 170-240 masl), 27\% from middle levels (250300 masl), and 9\% from near the top (350-400 masl). Fossils from Río Chalía are consistent with a Santacrucian Age (e.g., Cochlops muricatus, Nesodon imbricatus, Diadiaphorus majusculus, Neoremys australis, Perimys spp., etc.) and no taxa typical of other ages (e.g., Notohippidian, Colloncuran) have been recorded so far. They will help to address gaps in our understanding of the local response of vertebrate faunas to regional Andean uplift and its climatic effects and the worldwide climate warming during the mid-Miocene climate optimum and just prior to the global plunge in temperatures after about $14.75 \mathrm{Ma}$.

*Financial support by PICT 2013-0389; UNLP 11/N750; NGS CP-030R-17. 


\title{
SOUTH AMERICAN TURTLES AND THE CRETACEOUS-PALEOGENE EXTINCTION EVENT
}

\author{
E. VLACHOS ${ }^{1}$, E. RANDOLFE ${ }^{2}$, J. SterLI ${ }^{1}$ Y J.M. LeARDI ${ }^{3 *}$
}

${ }^{1}$ Consejo Nacional de Investigaciones Científicas y Técnicas (CONICET), Museo Paleontológico Egidio Feruglio, Av. Fontana 140, U9100GYO Trelew, Chubut, Argentina.evlacho@mef.org.ar; jsterli@mef.org.ar

${ }^{2}$ Centro de investigaciones en Ciencias de la Tierra (CICTERRA)-Consejo Nacional de Investigaciones Científicas y Técnicas (CONICET) and Universidad Nacional de Córdoba. Av. Vélez Sársfield 1611, X5016CGA Córdoba, Córdoba, Argentina. enrique.randolfe@hotmail.com

${ }^{3}$ Instituto de Estudios Andinos “Don Pablo Groeber" (IDEAN), Consejo Nacional de Investigaciones Científicas y Tecnológicas (CONCIET)-Universidad de Buenos Aires, Facultad de Ciencias Exactas y Naturales, Depto. de Biodiversidad y Biología Experimental. Intendente Güiraldes 2160, Ciudad Universitaria-Pabellón 2, C1428EGA Ciudad Autónoma de Buenos Aires, Argentina. juanmartinleardi@gmail.com

Turtles were always thought to have been unaffected by major extinction events that occurred on Earth, like the Cretaceous-Paleogene one that wiped out the non-avian dinosaurs. This thought was mainly based on genera counts across the $\mathrm{K} / \mathrm{Pg}$ boundary. The unprecedented growth of the South American turtle fossil record allows for a thorough analysis of the changes in the diversity of turtles in the continent, and to re-visit those ideas on their extinction. Our analysis suggests that turtles in South America reached their top diversity in the Late Cretaceous, only to immediately experience an important extinction event. The turtle diversity was immediately reduced in the boundary and continued its decrease during the entire Paleogene. By Oligocene, turtles in South America were reduced to a third of their Cretaceous diversity. This contribution was able to demonstrate for the first time evidence of K/Pg extinction in turtles. South American turtle diversity continued to suffer, but injections of biodiversity from Africa (arrival of testudinids) and North-Central America (GABI) introduced new clades to the continent. Besides the K/Pg extinction and its aftermath, we find that South American turtles have experienced other notable extinction events: two in the Early Cretaceous and another at the end of the Neogene. All of them, however, were accompanied by higher diversification and thus go largely unnoticed in the fossil record. The modern South American turtle fauna only took its shape in the last million years.

*Financial support by CONICET postdoctoral grant and a PICT -2016-2334 grant (EV); Instituto de Estudios Andinos Don Pablo Groeber R-264 contribution (JML).

\section{THE TRUE STRUCTURE OF THE INTERNATIONAL CODE OF ZOOLOGICAL NOMENCLATURE}

\section{E. VLACHOS ${ }^{1}$}

${ }^{1}$ Consejo Nacional de Investigaciones Científicas y Técnicas (CONICET). Museo Paleontológico Egidio Feruglio. Av. Fontana 140, U9100GYO Trelew, Chubut, Argentina.evlacho@mef.org.ar

To describe the various concepts of taxa in biology, evolution, and paleontology, scientists have developed various rules on how to create unique names for taxa. Zoological nomenclature is governed by the International Code of Zoological Nomenclature, a standard set of rules that govern the formation, publication, and application of the nomina of extant and extinct animals. This Code is the result of decades of discussions, workshops, publications and revisions by zoologists, formalized in editions of the Code, authored by the International Commission of Zoological Nomenclature. The structure and complexity of the Code have been attacked many times. I herein investigate the true structure of the Code, based on a novel network analysis. I explain how the Code is a complex network, and that any kind of publication in a form of continuous text is not possible to reflect its true structure. The network of the Code allows a better, in-depth understanding of the Code itself and is a tool that could help teaching, using and disseminating the Code. More importantly, this network is a powerful tool that allows identifying a priori the parts of the Code that could be potentially affected by upcoming amendment and revisions. All versions of the network of the Code will be made freely available for the users. This kind of analysis is not limited to nomenclature, as it could be applied to other fields that use such heavy and complicated textbooks. 


\title{
UN NUUEVO CROCODILOMORFO TEMPRANO (ARCHOSAURIA; PSEUDOSUCHIA) DE LA FOR- MACIÓN ISCHIGUALASTO (CARNIANO-NORIANO)
}

\author{
I. YÁñeZ ${ }^{1}$, D. POL ${ }^{2}$ Y R. MARTíneZ ${ }^{1}$ \\ ${ }^{1}$ Instituto y Museo de Ciencias Naturales, Facultad de Ciencias Exactas, Físicas y Naturales, Universidad Nacional de San Juan. Av. España \\ 400 (Norte), J5400DNQ San Juan, San Juan, Argentina.iyanez@unsj.edu.ar; martinez@unsj.edu.ar \\ Consejo Nacional de Investigaciones Científicas y Técnicas (CONICET). Museo Paleontológico "Egidio Feruglio". Av. Fontana 140, U9100GY0 \\ Trelew, Chubut, Argentina. dpol@mef.org.ar
}

Crocodylomorpha es un clado de arcosaurios que representa el único linaje de pseudosuquios que sobrevive al límite Triásico/Jurásico e incluye a los cocodrilos vivientes. Uno de los taxones más antiguos de este grupo es Trialestes romeri Reig, proveniente de la Formación Ischigualasto. En el presente trabajo se describe un nuevo crocodilomorfo no-crocodiliforme (PVSJ 717) proveniente de niveles inferiores del miembro Cancha de Bochas en los afloramientos australes de la Formación Ischigualasto (Carniano-Noriano) de San Juan. El espécimen está representado por varios elementos postcraneales (vértebras cervicales y dorsales, húmeros, radios, ulnas y un radial izquierdo) y posee un tamaño similar a T. romeri. Un análisis filogenético preliminar recupera a PVSJ 717 bien anidado en Crocodylomorpha en dos posiciones alternativas igualmente parsimoniosas: como grupo hermano de Terrestrisuchus gracilis Crush o de Dibothrosuchus elaphros Simmons, y el resto de los crocodilomorfos. La sinapomorfía que soporta su asignación a Crocodylomorpha es la presencia de un radial elongado. El nuevo espécimen se diferencia de Trialestes romeri por la presencia de fosas profundas en los laterales de los centros vertebrales cervicales, ápice de la cresta deltopectoral en una posición menor al 30\% de la longitud del húmero, extremo proximal del húmero expandido posteriormente y con forma de gancho, y radial con una proporción largo/ancho proximal mayor a tres. PVSJ 717 representaría la segunda especie de Crocodylomorpha conocida hasta el momento para la Formación Ischigualasto y permite obtener una mayor comprensión sobre la diversidad y disparidad morfológica de este grupo durante su evolución temprana.

\section{UN PARTICULAR EJEMPLAR DE PANOCHTHUS (XENARTHRA: GLYPTODONTIDAE) DEL PLEIS- TOCENO DE RÍO TERCERO, CÓRDOBA, ARGENTINA}

\author{
M. Zamorano ${ }^{1}$, F. Cuadrelli², J. Ochoa ${ }^{3}$, C. Luna², D. Barasoain ${ }^{2}$ y A.E. Zurita ${ }^{2}$
}

${ }^{1}$ División Paleontología de Vertebrados, Museo de La Plata, Facultad de Ciencias Naturales y Museo, Universidad Nacional de La Plata. Paseo del Bosque s/n, B1900FWA La Plata, Buenos Aires, Argentina. marzamorano@fcnym.unlp.edu.ar

${ }^{2}$ Centro de Ecología Aplicada del Litoral (CECOAL)-Consejo Nacional de Investigaciones Científicas y Tecnológicas (CONICET) y Universidad Nacional del Nordeste. Ruta 5, km 2,5, 3400 Corrientes, Argentina.f.cuadrelli@gmail.com; carlosaluna@hotmail.com; danielbarasoain@gmail.com; aezurita74@yahoo.com.ar

3Museo Regional "Florentino Ameghino". Casa "Villa Elisa", 5850 Río Tercero, Córdoba, Argentina. javiergochoa@hotmail.com

Panochthus Burmeister es uno de los géneros de gliptodóntidos con mayor distribución latitudinal del Pleistoceno de América del Sur. Las últimas revisiones sugirieren que incluye seis especies: $P$. intermedius Lydekker, $P$. subintermedius Castellanos, P. tuberculatus Owen, P. frenzelianus Ameghino, P. greslebini Castellanos y P. jaguaribensis Moreira; recientemente se reconoció una nueva especie, $P$. hipsilis Zurita, Zamorano, Scillato-Yané, Fidel, Iriondo y Gillette. En esta contribución se presenta un ejemplar casi completo y en un excelente estado de preservación que procede de sedimentos expuestos en las márgenes del río Ctalamochita en los alrededores de la ciudad de Río Tercero (Córdoba, Argentina) interpretados como depósitos de llanura de inundación asignados tentativamente a la Formación Toro Muerto de edad Pleistoceno Medio. Su morfología general permite su asignación a Panochthus, aunque muestra ciertas particularidades: a) cráneo similar al de $P$. tuberculatus, pero llamativamente la región fronto-nasal está menos inclinada hacia abajo, y la escotadura de la marinaes de contorno más circular; b) el tubo caudal presenta en su extremo distal solo una figura apical, como en $P$. subintermedius y $P$. greslebini, y a diferencia de $P$. tuberculatus, $P$. intermedius y $P$. jaguaribensis. Por otra parte, el escudete cefálico, la coraza dorsal y el esqueleto apendicular son similares a $P$. tuberculatus. Hasta el momento, la paleofauna asociada (e.g., Catonyx tarijensis) indica claramente una antigüedad pleistocena para este ejemplar. Posteriores estudios podrán dilucidar si las diferencias observadas con las especies pampeanas indican que se trata de un nuevo taxón o bien deben ser consideradas como variaciones intraespecíficas. 